Supporting Information for

\title{
Cu-Catalyzed Three-Component Carboamination of Alkenes
}

\author{
Samuel N. Gockel, ${ }^{\ddagger}$ Travis L. Buchanan, ${ }^{\ddagger}$ Kami L. Hull \\ Department of Chemistry, University of Illinois at Urbana-Champaign, 600 S. Mathews Avenue, Urbana, IL \\ 61801
}

Table of Contents

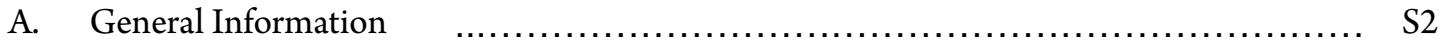

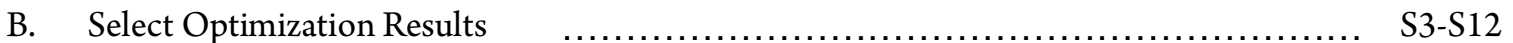

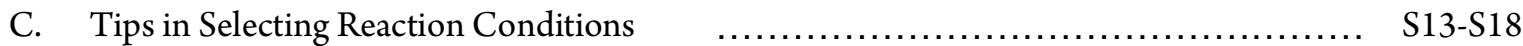

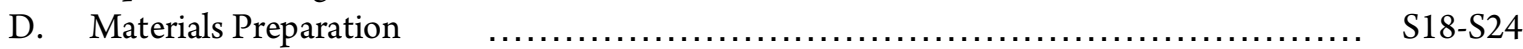

E. Experimental Procedure, Isolation, and Characterization $\quad \ldots \ldots \ldots \ldots \ldots \ldots \ldots \ldots \ldots \ldots \ldots$ S25-S51

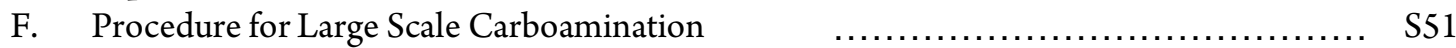

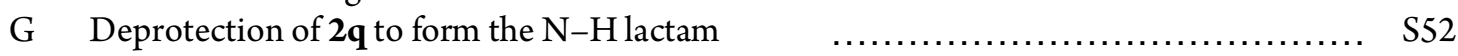

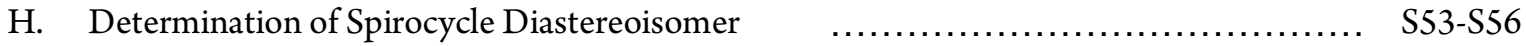

I. Additional Mechanistic Information and Catalytic Cycle $\quad \ldots \ldots \ldots \ldots \ldots \ldots \ldots \ldots \ldots$ S57-S58

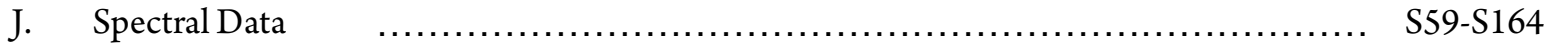




\section{A. General Information}

General Experimental Procedures: Air- and moisture-sensitive reagents were handled in a nitrogenfilled glovebox (working oxygen level $\sim 0.1 \mathrm{ppm}$ ). Column chromatography was performed with silica gel from SiliCycle $(40-63 \mu \mathrm{m})$ with a column mixed as a slurry with the eluent and was packed, rinsed, and run under air pressure. Analytical thin-layer chromatography (TLC) was performed on precoated glass silica gel plates (by EMD Chemicals Inc.) with F-254 indicator with visualization by short wave (254 $\mathrm{nm}$ ) ultraviolet light. Distillations were performed using a $3 \mathrm{~cm}$ short-path column under reduced pressure.

Instrumentation: ${ }^{1} \mathrm{H}$ and ${ }^{13} \mathrm{C}$ NMR spectra were recorded on Varian Unity $500 \mathrm{MHz}\left(125 \mathrm{MHz}\right.$ for ${ }^{13} \mathrm{C}$, 470 for $\left.{ }^{19} \mathrm{~F}\right)$ and Bruker Ascend $500 \mathrm{MHz}\left(125 \mathrm{MHz}\right.$ for ${ }^{13} \mathrm{C}, 470$ for $\left.{ }^{19} \mathrm{~F}\right)$ spectrometers. Spectra were referenced to the residual solvent peak of $\mathrm{CDCl}_{3}$ unless otherwise noted. Chemical shifts were reported in parts per million and multiplicities are as indicated: $s$ (singlet,) $d$ (doublet,) $t$ (triplet,) q (quartet,) p (pentet,) $\mathrm{m}$ (multiplet,) and br (broad). Coupling constants, $\mathrm{J}$, are reported in Hertz and integration is provided, along with assignments, as indicated. Gas Chromatography (GC) was performed on a Shimadzu GC-2010 Plus gas chromatograph with SHRXI-MS- $15 \mathrm{~m}$ x $0.25 \mathrm{~mm} \times 0.25 \mu \mathrm{m}$ column with nitrogen carrier gas and a flame ionization detector (FID). The glove box, MBraun LABmaster sp, was maintained under nitrogen atmosphere. Melting points were recorded on a Thomas Hoover capillary melting point apparatus and are uncorrected.

Materials: Solvents used for extraction and column chromatography were reagent grade and used as received. Reaction solvents tetrahydrofuran (Fisher, unstabilized HPLC ACS grade), toluene (Fisher, optima ACS grade), 1,4-dioxane (Fisher, certified ACS), acetonitrile (Fisher, HPLC grade), and hexanes (Fisher, ACS HPLC grade) were dried on a Pure Process Technology Glass Contour Solvent Purification System using activated Stainless Steel columns while following manufacturer's recommendations for solvent preparation and dispensation unless otherwise noted. 1,2-Dichloroethane was purchased from Aldrich Chemical in a sure-sealed bottle, which was transferred into a glove box under nitrogen immediately upon receipt. Amines were distilled and degassed using the freeze-pumpthaw method upon receipt (unless otherwise noted) and stored under nitrogen in a glove box. Styrene was purchase from Aldrich Chemical and was washed with $5 \mathrm{M} \mathrm{NaOH}$ to remove inhibitors. It was then dried with $\mathrm{MgSO}_{4}$ pulled through a plug of neutral alumina and degassed using the freeze-pump-thaw method. It was stored at $-40{ }^{\circ} \mathrm{C}$ under nitrogen in a glove box. Unless otherwise shown, all noncommercial substrates were prepared according to the known literature procedures. 


\section{B. Select Optimization Results}

Table S1. Varying the Metal Salt. ${ }^{a}$

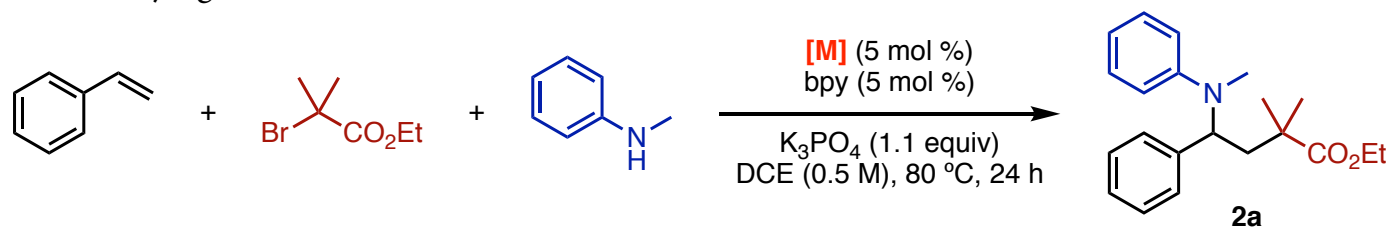

\begin{tabular}{ccc}
\hline Entry & {$[\mathrm{M}]$} & \% yield $\mathbf{2 a}^{b}$ \\
\hline 1 & $\mathrm{Cu}(\mathrm{OTf})_{2}$ & 87 \\
2 & $\mathrm{FeCl}_{2}$ & 45 \\
3 & $\mathrm{Pd}(\mathrm{OAc})_{2}$ & 6 \\
4 & $\mathrm{CoCl}_{2}$ & 0 \\
5 & $\mathrm{NiBr}_{2} \cdot$ glyme & 0 \\
\hline
\end{tabular}

a. In a nitrogen-filled glovebox an oven-dried 4-mL reaction vial was charged with metal salt ( $5 \mu \mathrm{mol}, 5$ $\mathrm{mol} \%), \mathrm{K}_{3} \mathrm{PO}_{4}(23.3 \mathrm{mg}, 0.11 \mathrm{mmol}, 1.1$ equiv), and a stir bar. $200 \mu \mathrm{L}$ of a DCE stock solution containing 2,2'-bipyridine $(0.025 \mathrm{M}, 5 \mathrm{~mol} \%)$ was added. This was followed by ethyl 2-bromo-2methylpropanoate $(29.4 \mu \mathrm{L}, 0.2 \mathrm{mmol}, 2$ equiv), $N$-methylaniline ( $11.0 \mu \mathrm{L}, 0.1 \mathrm{mmol}, 1$ equiv), and styrene ( $11.5 \mu \mathrm{L}, 0.1 \mathrm{mmol}, 1$ equiv). The vial was sealed with a Teflon-coated screw cap, removed from the glovebox, and stirred at $660 \mathrm{rpm}$ at $80^{\circ} \mathrm{C}$ for $24 \mathrm{~h} .{ }^{b}$. In situ yield determined by GC analysis with comparison to undecane $(10 \mu \mathrm{L})$ as an internal standard. 
Table S2. Varying the Copper Salt. ${ }^{a}$

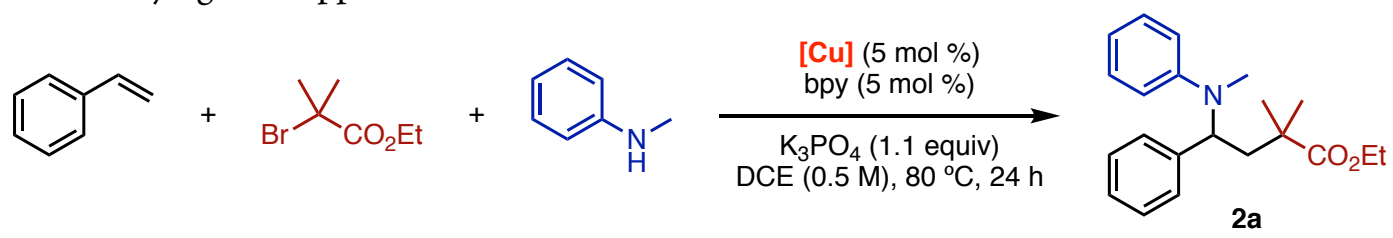

\begin{tabular}{ccc}
\hline Entry & {$[\mathrm{Cu}]$} & \% yield $\mathbf{2 a}^{b}$ \\
\hline 1 & None & 0 \\
2 & $\mathrm{Cu}(\mathrm{OTf})_{2}$ & 87 \\
3 & $\mathrm{Cu}(\mathrm{OAc})_{2}$ & 39 \\
4 & $\mathrm{CuCl}_{2}$ & 32 \\
5 & $\mathrm{CuCl}$ & 63 \\
6 & $\mathrm{CuI}$ & 55 \\
\hline
\end{tabular}

${ }^{a .}$ In a nitrogen-filled glovebox an oven-dried 4-mL reaction vial was charged with $\mathrm{Cu}$ salt ( $5 \mu \mathrm{mol}, 5$ $\mathrm{mol} \%), \mathrm{K}_{3} \mathrm{PO}_{4}(23.3 \mathrm{mg}, 0.11 \mathrm{mmol}, 1.1$ equiv), and a stir bar. $200 \mu \mathrm{L}$ of a DCE stock solution containing 2,2'-bipyridine $(0.025 \mathrm{M}, 5 \mathrm{~mol} \%)$ was added. This was followed by ethyl 2-bromo-2methylpropanoate $(29.4 \mu \mathrm{L}, 0.2 \mathrm{mmol}, 2$ equiv), $N$-methylaniline ( $11.0 \mu \mathrm{L}, 0.1 \mathrm{mmol}, 1$ equiv), and styrene ( $11.5 \mu \mathrm{L}, 0.1 \mathrm{mmol}, 1$ equiv). The vial was sealed with a Teflon-coated screw cap, removed from the glovebox, and stirred at $660 \mathrm{rpm}$ at $80^{\circ} \mathrm{C}$ for $24 \mathrm{~h} .{ }^{b}$. In situ yield determined by GC analysis with comparison to undecane $(10 \mu \mathrm{L})$ as an internal standard. 
Table S3. Ligand Screen. ${ }^{a}$

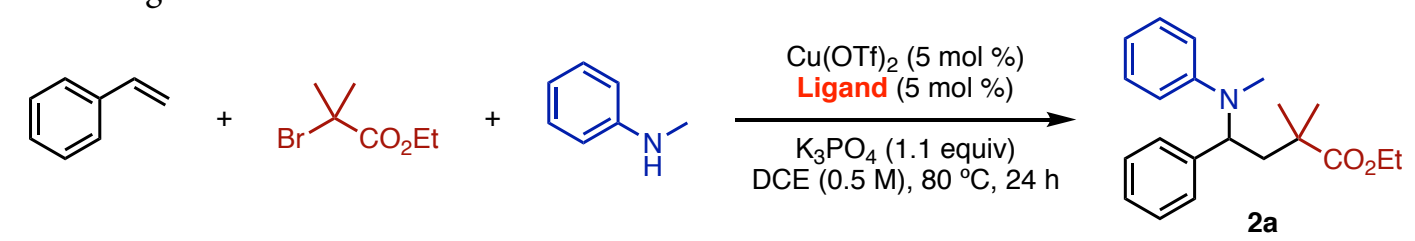

\begin{tabular}{ccc}
\hline Entry & Ligand & \% yield $\mathbf{2 a}^{b}$ \\
\hline 1 & 2,2'-bipyridine & 87 \\
2 & phen & 89 \\
3 & dtb2,2'-bipyridine & 91 \\
4 & TMEDA $^{b}$ MeN $^{c}$ & 45 \\
5 & Me $^{c}$ & 50 \\
6 & PMDTA $^{c}$ & 79 \\
7 & TEA & 4 \\
8 & $(S, S)-$ Ph-PyBox $^{c}$ & 19 \\
9 & PEPA $^{c}$ & 19 \\
10 & DPEPA $^{c}$ & 39 \\
11 & TPEN $^{c}$ & 82 \\
12 & DMAMP $^{c}$ & 81 \\
13 & TPA $^{c}$ & 88 \\
14 & PPh $_{3}$ & 1 \\
15 & None & 2 \\
\hline
\end{tabular}

${ }^{a .}$ In a nitrogen-filled glovebox an oven-dried 4-mL reaction vial was charged with $\mathrm{Cu}(\mathrm{OTf})_{2}(1.8 \mathrm{mg}, 5$ $\mu \mathrm{mol}, 5 \mathrm{~mol} \%$ ), ligand (if solid, $5 \mathrm{~mol} \%), \mathrm{K}_{3} \mathrm{PO}_{4}(23.3 \mathrm{mg}, 0.11 \mathrm{mmol}, 1.1$ equiv), and a stir bar. $200 \mu \mathrm{L}$ of DCE was added. This was followed by ligand (if liquid, $5 \mathrm{~mol} \%$ ), ethyl 2-bromo-2-methylpropanoate (29.4 $\mu \mathrm{L}, 0.2 \mathrm{mmol}, 2$ equiv), $N$-methylaniline ( $11.0 \mu \mathrm{L}, 0.1 \mathrm{mmol}, 1$ equiv), and styrene (11.5 $\mu \mathrm{L}, 0.1$ mmol, 1 equiv). The vial was sealed with a Teflon-coated screw cap, removed from the glovebox, and

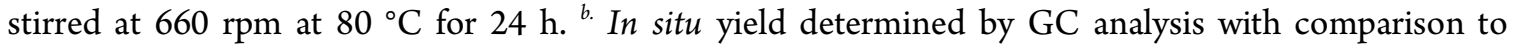
undecane $(10 \mu \mathrm{L})$ as an internal standard. ${ }^{c}$. See below for ligand structure: 
<smiles>CN(C)CCN(CCN(C)C)CCN(C)C</smiles><smiles>CN(C)CCN(C)CCN(C)C</smiles><smiles>c1ccc([C@H]2COC(c3cccc(C4=N[C@H](c5ccccc5)CO4)n3)=N2)cc1</smiles><smiles>c1ccc(CCNCc2ccccn2)nc1</smiles>

$\mathrm{Me}_{6}$ TREN

PMDTA

(S,S)-Ph-PyBox

PEPA<smiles>c1ccc(CCN(CCc2ccccn2)Cc2ccccn2)nc1</smiles><smiles>c1ccc(CN(CCN(Cc2ccccn2)Cc2ccccn2)Cc2ccccn2)nc1</smiles>

DPEPA

TPEN<smiles>CN(C)Cc1cccc(CN(C)C)n1</smiles>

DMAMP<smiles>c1ccc(CN(Cc2ccccn2)Cc2ccccn2)nc1</smiles>

TPA

Table S4. $\mathrm{Cu}(\mathrm{OTf})_{2}: 2,2^{\prime}$-bipyridine Ratio. ${ }^{a}$<smiles>C=Cc1ccccc1</smiles>
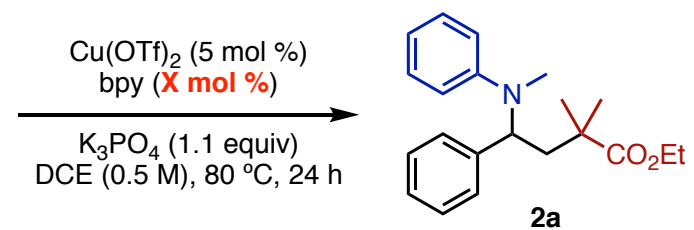

\begin{tabular}{ccc}
\hline Entry & mol \% 2,2'-bipyridine & \% yield $\mathbf{2 a}^{b}$ \\
\hline 1 & 2.5 & 95 \\
2 & 5 & 87 \\
3 & 10 & 46 \\
4 & 20 & 62 \\
5 & 50 & 79 \\
6 & 100 & 75 \\
\hline
\end{tabular}

\footnotetext{
${ }^{a .}$ In a nitrogen-filled glovebox an oven-dried 4-mL reaction vial was charged with $\mathrm{Cu}(\mathrm{OTf})_{2}(1.8 \mathrm{mg}, 5$ $\mu \mathrm{mol}, 5 \mathrm{~mol} \%), \mathrm{K}_{3} \mathrm{PO}_{4}(23.3 \mathrm{mg}, 0.11 \mathrm{mmol}, 1.1$ equiv), and a stir bar. $200 \mu \mathrm{L}$ of a DCE stock solution containing 2,2'-bipyridine (2.5-100 mol \%) was added. This was followed by ethyl 2-bromo-2methylpropanoate $(29.4 \mu \mathrm{L}, 0.2 \mathrm{mmol}, 2$ equiv), $N$-methylaniline ( $11.0 \mu \mathrm{L}, 0.1 \mathrm{mmol}, 1$ equiv), and styrene ( $11.5 \mu \mathrm{L}, 0.1 \mathrm{mmol}, 1$ equiv). The vial was sealed with a Teflon-coated screw cap, removed from the glovebox, and stirred at $660 \mathrm{rpm}$ at $80{ }^{\circ} \mathrm{C}$ for $24 \mathrm{~h}$. ${ }^{b}$. In situ yield determined by GC analysis with comparison to undecane $(10 \mu \mathrm{L})$ as an internal standard.
} 
Table S5. Varying the Copper Loading. ${ }^{a}$

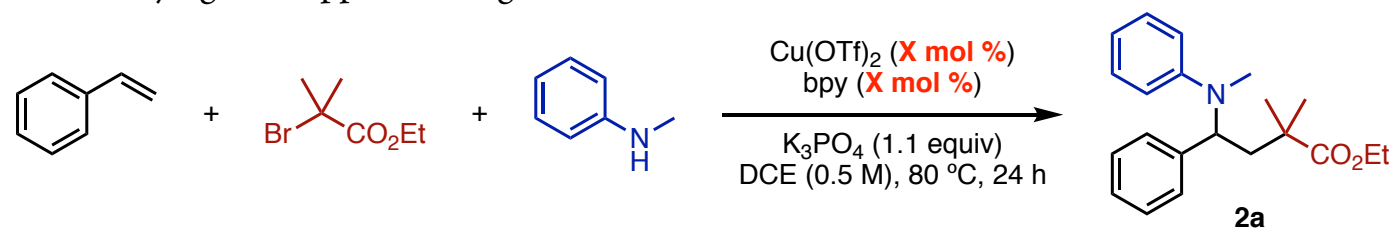

\begin{tabular}{ccc}
\hline Entry & $\mathrm{Cu}(\mathrm{OTf})_{2}$ loading $(\mathrm{X} \mathrm{mol} \%)$ & \% yield 2a $^{b}$ \\
\hline 1 & 1 & 19 \\
2 & 2.5 & 83 \\
3 & 5 & 87 \\
4 & 7.5 & 89 \\
5 & 10 & 86 \\
6 & 20 & 85 \\
\hline
\end{tabular}

${ }^{a}$ In a nitrogen-filled glovebox an oven-dried 4-mL reaction vial was charged with $\mathrm{Cu}(\mathrm{OTf})_{2}(\mathrm{X} \mathrm{mol} \%)$, $\mathrm{K}_{3} \mathrm{PO}_{4}(23.3 \mathrm{mg}, 0.11 \mathrm{mmol}, 1.1$ equiv), and a stir bar. $200 \mu \mathrm{L}$ of a DCE stock solution containing 2,2'bipyridine (0.005X M in 2,2'-bipyridine, $\mathrm{X} \mathrm{mol} \%$ ) was added. This was followed by ethyl 2-bromo-2methylpropanoate $(29.4 \mu \mathrm{L}, 0.2 \mathrm{mmol}, 2$ equiv), $N$-methylaniline ( $11.0 \mu \mathrm{L}, 0.1 \mathrm{mmol}, 1$ equiv), and styrene $(11.5 \mu \mathrm{L}, 0.1 \mathrm{mmol}, 1$ equiv). The vial was sealed with a Teflon-coated screw cap, removed from the glovebox, and stirred at $660 \mathrm{rpm}$ at $80^{\circ} \mathrm{C}$ for $24 \mathrm{~h} .{ }^{b}$. In situ yield determined by GC analysis with comparison to undecane $(10 \mu \mathrm{L})$ as an internal standard. 
Table S6. Base. ${ }^{a}$

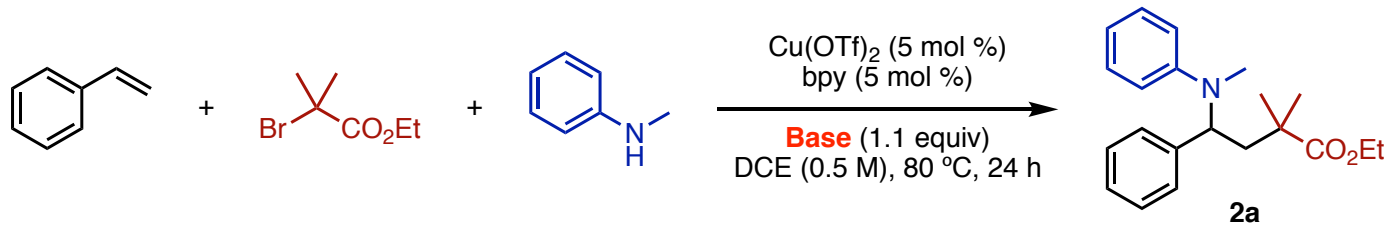

\begin{tabular}{ccc}
\hline Entry & Base & \% yield $\mathbf{2 a}^{b}$ \\
\hline 1 & $\mathrm{~K}_{3} \mathrm{PO}_{4}$ & 87 \\
2 & $\mathrm{Cs}_{2} \mathrm{CO}_{3}$ & 76 \\
3 & $\mathrm{NaHCO}_{3}$ & 55 \\
4 & $\mathrm{LiO}^{t} \mathrm{Bu}$ & 30 \\
5 & $\mathrm{CsF}$ & 52 \\
6 & $\mathrm{None}$ & 46 \\
\hline
\end{tabular}

${ }^{a .}$ In a nitrogen-filled glovebox an oven-dried 4-mL reaction vial was charged with $\mathrm{Cu}(\mathrm{OTf})_{2}(1.8 \mathrm{mg}, 5$ $\mu \mathrm{mol}, 5 \mathrm{~mol} \%)$, Base (1.1 equiv), and a stir bar. $200 \mu \mathrm{L}$ of a DCE stock solution containing 2,2'bipyridine $(0.025 \mathrm{M}, 5 \mathrm{~mol} \%)$ was added. This was followed by ethyl 2-bromo-2-methylpropanoate (29.4 $\mu \mathrm{L}, 0.2 \mathrm{mmol}, 2$ equiv), $N$-methylaniline ( $11.0 \mu \mathrm{L}, 0.1 \mathrm{mmol}, 1$ equiv), and styrene ( $11.5 \mu \mathrm{L}, 0.1$ mmol, 1 equiv). The vial was sealed with a Teflon-coated screw cap, removed from the glovebox, and stirred at $660 \mathrm{rpm}$ at $80^{\circ} \mathrm{C}$ for $24 \mathrm{~h} .{ }^{b}$. In situ yield determined by GC analysis with comparison to undecane $(10 \mu \mathrm{L})$ as an internal standard. 
Table S7. Solvent. ${ }^{a}$

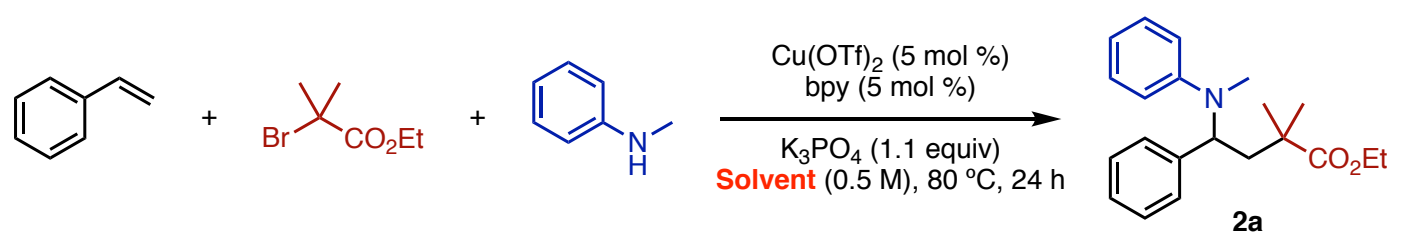

\begin{tabular}{ccc}
\hline Entry & Solvent & \% yield $\mathbf{2 a}^{b}$ \\
\hline 1 & DCE & 87 \\
2 & THF & 77 \\
3 & DME & 71 \\
4 & DMA & 28 \\
5 & Benzene & 77 \\
5 & PhMe & 15 \\
7 & MeCN & 79 \\
\hline
\end{tabular}

\footnotetext{
a. In a nitrogen-filled glovebox an oven-dried 4-mL reaction vial was charged with $\mathrm{Cu}(\mathrm{OTf})_{2}(1.8 \mathrm{mg}, 5$ $\mu \mathrm{mol}, 5.0 \mathrm{~mol} \%), 2,2$ '-bipyridine ( $0.8 \mathrm{mg}, 5 \mu \mathrm{mol}, 5 \mathrm{~mol} \%), \mathrm{K}_{3} \mathrm{PO}_{4}(23.3 \mathrm{mg}, 1.1$ equiv), and a stir bar. $200 \mu \mathrm{L}$ of solvent was added. This was followed by ethyl 2-bromo-2-methylpropanoate (29.4 $\mu \mathrm{L}, 0.2$ mmol, 2 equiv), $N$-methylaniline ( $11.0 \mu \mathrm{L}, 0.1 \mathrm{mmol}, 1$ equiv), and styrene ( $11.5 \mu \mathrm{L}, 0.1 \mathrm{mmol}, 1$ equiv). The vial was sealed with a Teflon-coated screw cap, removed from the glovebox, and stirred at $660 \mathrm{rpm}$ at $80^{\circ} \mathrm{C}$ for $24 \mathrm{~h} .{ }^{b}$ In situ yield determined by GC analysis with comparison to undecane $(10 \mu \mathrm{L})$ as an internal standard.
} 
Table S8. Concentration. ${ }^{a}$

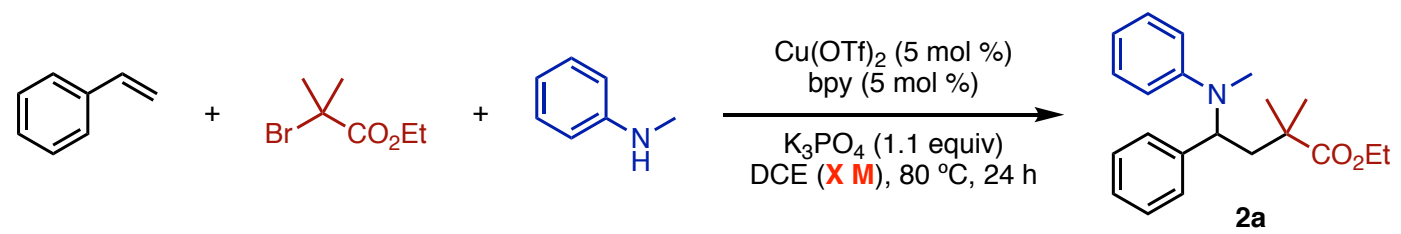

\begin{tabular}{ccc}
\hline Entry & Concentration & \% yield 2a ${ }^{b}$ \\
\hline 1 & $2.0 \mathrm{M}$ & 88 \\
2 & $1.0 \mathrm{M}$ & 86 \\
3 & $0.5 \mathrm{M}$ & 87 \\
4 & $0.33 \mathrm{M}$ & 39 \\
5 & $0.20 \mathrm{M}$ & 44 \\
6 & $0.10 \mathrm{M}$ & 76 \\
\hline
\end{tabular}

${ }^{a .}$ In a nitrogen-filled glovebox an oven-dried 4-mL reaction vial was charged with $\mathrm{Cu}(\mathrm{OTf})_{2}(1.8 \mathrm{mg}, 5$ $\mu \mathrm{mol}, 5 \mathrm{~mol} \%), \mathrm{K}_{3} \mathrm{PO}_{4}(23.3 \mathrm{mg}, 1.1 \mathrm{equiv})$, and a stir bar. $50 \mu \mathrm{L}$ of a DCE stock solution containing 2,2'-bipyridine (0.10 M, $5 \mu \mathrm{mol}, 5 \mathrm{~mol} \%)$, was added followed by additional DCE to achieve the desired concentration. This was followed by ethyl 2-bromo-2-methylpropanoate $(29.4 \mu \mathrm{L}, 0.2 \mathrm{mmol}, 2$ equiv), $N$-methylaniline ( $11.0 \mu \mathrm{L}, 0.1 \mathrm{mmol}, 1$ equiv), and styrene ( $11.5 \mu \mathrm{L}, 0.1 \mathrm{mmol}, 1$ equiv). The vial was sealed with a Teflon-coated screw cap, removed from the glovebox, and stirred at $660 \mathrm{rpm}$ at $80^{\circ} \mathrm{C}$ for 24 h. ${ }^{b}$ In situ yield determined by GC analysis with comparison to undecane $(10 \mu \mathrm{L})$ as an internal standard. 
Table S9. Reagent Loadings. ${ }^{a}$

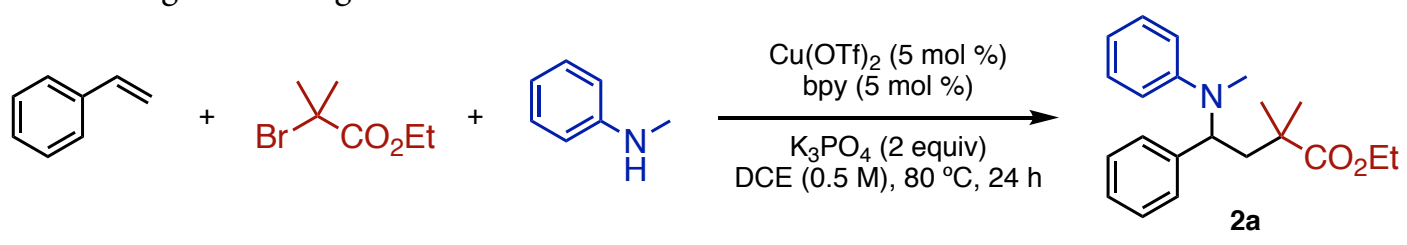

\begin{tabular}{cccc}
\hline Entry & Equiv styrene & Equiv ethyl 2-bromo-2-methylpropanoate & \% yield 2a ${ }^{b}$ \\
\hline 1 & 1 & 2 & 64 \\
2 & 3 & 2 & 87 \\
3 & 5 & 2 & 99 \\
\hline 4 & 1 & 1 & 70 \\
5 & 1 & 3 & 85 \\
6 & 1 & 5 & $>99$ \\
\hline
\end{tabular}

${ }^{a .}$ In a nitrogen-filled glovebox an oven-dried 4-mL reaction vial was charged with $\mathrm{Cu}(\mathrm{OTf})_{2}(1.8 \mathrm{mg}, 5$ $\mu \mathrm{mol}, 5 \mathrm{~mol} \%), \mathrm{K}_{3} \mathrm{PO}_{4}(46.6 \mathrm{mg}, 0.20 \mathrm{mmol}, 2.0$ equiv), and a stir bar. $200 \mu \mathrm{L}$ of a DCE stock solution containing 2,2'-bipyridine $(0.025 \mathrm{M}, 5 \mathrm{~mol} \%)$ was added. This was followed by ethyl 2-bromo-2methylpropanoate (1-5 equiv), $N$-methylaniline ( $11.0 \mu \mathrm{L}, 0.1 \mathrm{mmol}, 1$ equiv), and styrene (1-5 equiv). The vial was sealed with a Teflon-coated screw cap, removed from the glovebox, and stirred at $660 \mathrm{rpm}$ at $80^{\circ} \mathrm{C}$ for $24 \mathrm{~h} .{ }^{b}$ In situ yield determined by GC analysis with comparison to undecane $(10 \mu \mathrm{L})$ as an internal standard. 
Table S10. Impact of Water. ${ }^{a}$
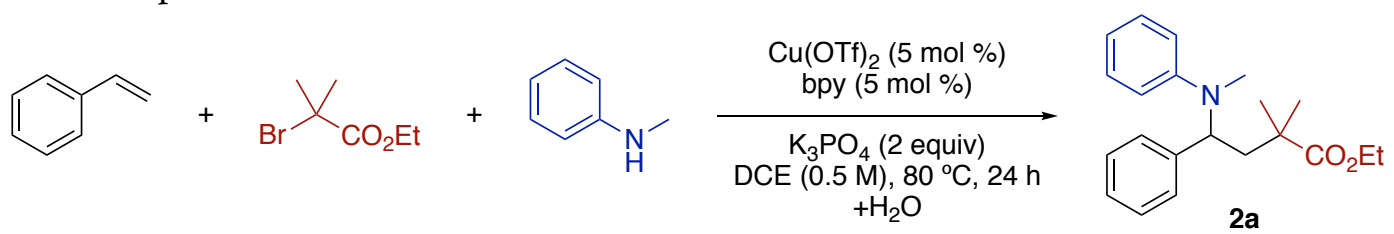

\begin{tabular}{cccc}
\hline Entry & Water Added & Note & $\%$ yield 2a \\
\hline 1 & no water added & & 87 \\
2 & $1.0 \mu \mathrm{L}, 0.60$ equiv & oven-dried $\mathrm{K}_{3} \mathrm{PO}_{4}$ and vial & 78 \\
3 & $3.0 \mu \mathrm{L}, 1.7$ equiv & & 3 \\
4 & $7.0 \mu \mathrm{L}, 3.9$ equiv & & 2 \\
\hline 5 & \multirow{2}{*}{ no water added } & $\mathrm{K}_{3} \mathrm{PO}_{4}$ was not oven-dried & 74 \\
6 & & vial was not oven-dried & 82 \\
\hline
\end{tabular}

${ }^{a}$. In a nitrogen-filled glovebox an oven-dried 4-mL reaction vial with a Teflon-cap was charged with $\mathrm{Cu}(\mathrm{OTf})_{2}(1.8 \mathrm{mg}, 5 \mu \mathrm{mol}, 5 \mathrm{~mol} \%), \mathrm{K}_{3} \mathrm{PO}_{4}(23.3 \mathrm{mg}, 0.10 \mathrm{mmol}, 1.0$ equiv $)$, and a stir bar. $200 \mu \mathrm{L}$ of a DCE stock solution containing 2,2'-bipyridine $(0.025 \mathrm{M}, 5 \mathrm{~mol} \%)$ was added. This was followed by ethyl 2-bromo-2-methylpropanoate $(29.4 \mu \mathrm{L}, 0.2 \mathrm{mmol}, 2$ equiv), $N$-methylaniline $(11.0 \mu \mathrm{L}, 0.1 \mathrm{mmol}, 1$ equiv), and styrene ( $11.5 \mu \mathrm{L}, 0.1 \mathrm{mmol}, 1$ equiv). The vial was sealed with a Teflon-coated screw cap, removed from the glovebox, and water was added through the septum $(0-15 \mu \mathrm{L})$. The mixture was stirred at $660 \mathrm{rpm}$ at $80^{\circ} \mathrm{C}$ for $24 \mathrm{~h} .{ }^{b}$ In situ yield determined by GC analysis with comparison to undecane (10 $\mu \mathrm{L})$ as an internal standard. 


\section{Tips in Selecting Reaction Conditions}

The general conditions for the reaction for vinylarenes and aliphatic alkenes are shown in eqs. 1 and 2 below.

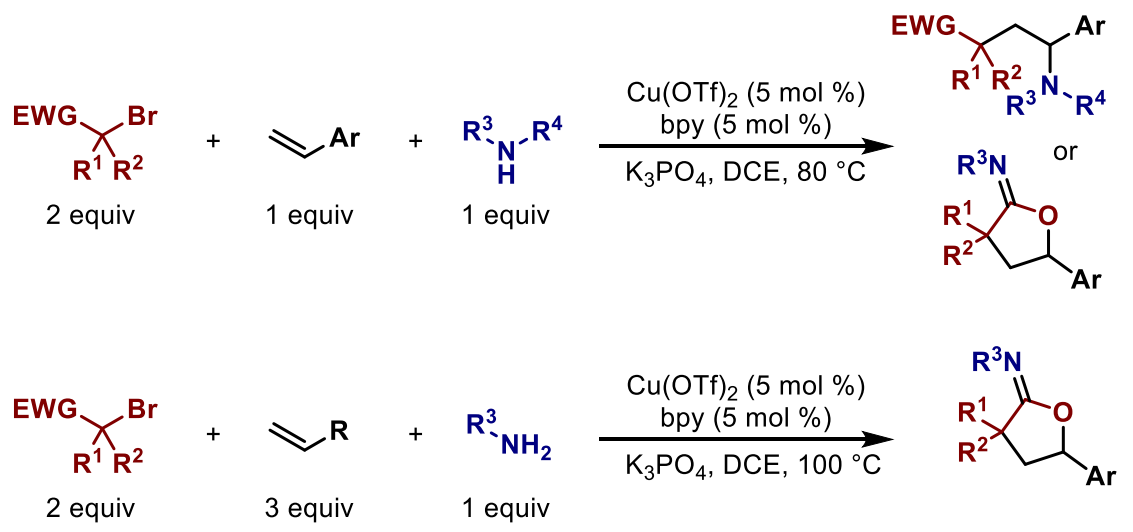

For some substrates, it was observed that the general conditions were not optimal, and fine-tuning was conducted to arrive at more efficient conditions. While optimizing and exploring the scope of this reaction, it was found that the products could often be formed under a wide range of conditions. This is 
important because it provides an array of optimization tools. Many substrates, including ones that have not been shown in this manuscript, can be made to work efficiently in this reaction.

In such cases where yield of the desired product is low, we recommend screening these parameters to improve yield. The parameters are discussed in order of the magnitude by which they influence reaction efficiency.

\section{Ligands and Ligand Loading:}

The ligand employed in the reaction is of critical importance. In cases where a substrate combination is not performing well, screening ligands can often quickly lead to high yielding conditions. We recommend exploring the ligands shown below.<smiles>CNCCNC</smiles>

DMEDA

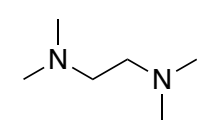

TMEDA

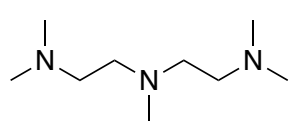

PMDETA<smiles>CN(C)CCN(CCN(C)C)CCN(C)C</smiles>

$\mathrm{Me}_{6}$ TREN<smiles>c1ccc(CN(CCN(Cc2ccccn2)Cc2ccccn2)Cc2ccccn2)nc1</smiles>

TPEN

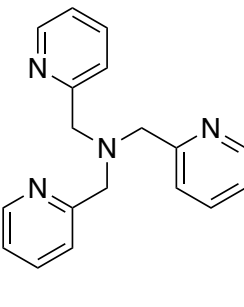

TPA

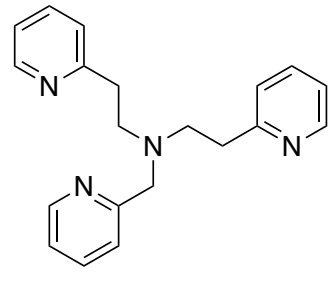

DPEPA<smiles>c1ccc(CCNCCc2ccccn2)nc1</smiles>

PEPA

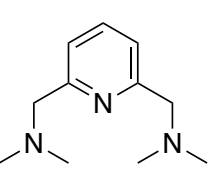

DMAMP

Once a ligand that works well has been identified, we recommend varying the $\mathrm{Cu}$ :ligand ratio. In some cases, such as for product $\mathbf{5 w}$ a 1:2 ratio of $\mathrm{Cu}$ :ligand led to significantly higher yields (i.e. $~ 37 \%$ yield with $1: 1 \mathrm{Cu}$ :ligand ratio, $61 \%$ yield with $1: 2 \mathrm{Cu}$ :ligand ratio).

\section{Reagent Loadings:}

Adding excess of the alkene or electrophile substrate often leads to significant improvements in yield. Up to 5.0 equiv of one or both of these coupling partners may be required to achieve synthetically useful yields. We recommend screening 1.0-5.0 equiv of each.

In some cases, such as for product $\mathbf{5 w}$, a lower loading of the electrophile led to higher yields (i.e. $~ 48 \%$ yield with 2.0 equiv and $61 \%$ yield with 1.0 equiv) and higher alkene loadings significantly helped (i.e. $\sim 24 \%$ yield with 1.0 equiv and $61 \%$ yield with 5.0 equiv).

\section{Temperature:}

The general conditions provide a good starting point when optimizing reaction temperature. In some cases, particularly when substrates possess a potentially reactive functionality, such as a $1^{\circ}$ aliphatic bromide (2ad), terminal epoxide (2ae-af), or aliphatic ketone (2ag), the reaction performs better at lower temperatures. In cases when the substrate does not bear any fragile functionality, a higher temperature can improve yield.

\section{Presence of Water:}

The reaction is sensitive to water (Table S10 above). It was found that spiking the reaction with a small amount of water led to significantly diminished yields. If the reaction is not performing well, oven-dry the 
vials, stir bars, and any implements used to weigh out reagents. $\mathrm{K}_{3} \mathrm{PO}_{4}$ is hygroscopic and was always oven-dried before use. $\mathrm{Cu}(\mathrm{OTf})_{2}$ and $\mathrm{DCE}$ were used as received and were transferred into a dry $(<0.1$ ppm $\mathrm{H}_{2} \mathrm{O}$ ) glove-box immediately following receipt.

\section{General Notes:}

When approaching the use of this reaction with a target product in mind, we recommend consulting the table below (Table S11), identifying a structurally similar product, and using those conditions as a starting point. From there, the parameters discussed above can be explored to further optimize the conditions.

Table S11: This section is meant to serve as a quick reference for which conditions were used with which substrates.

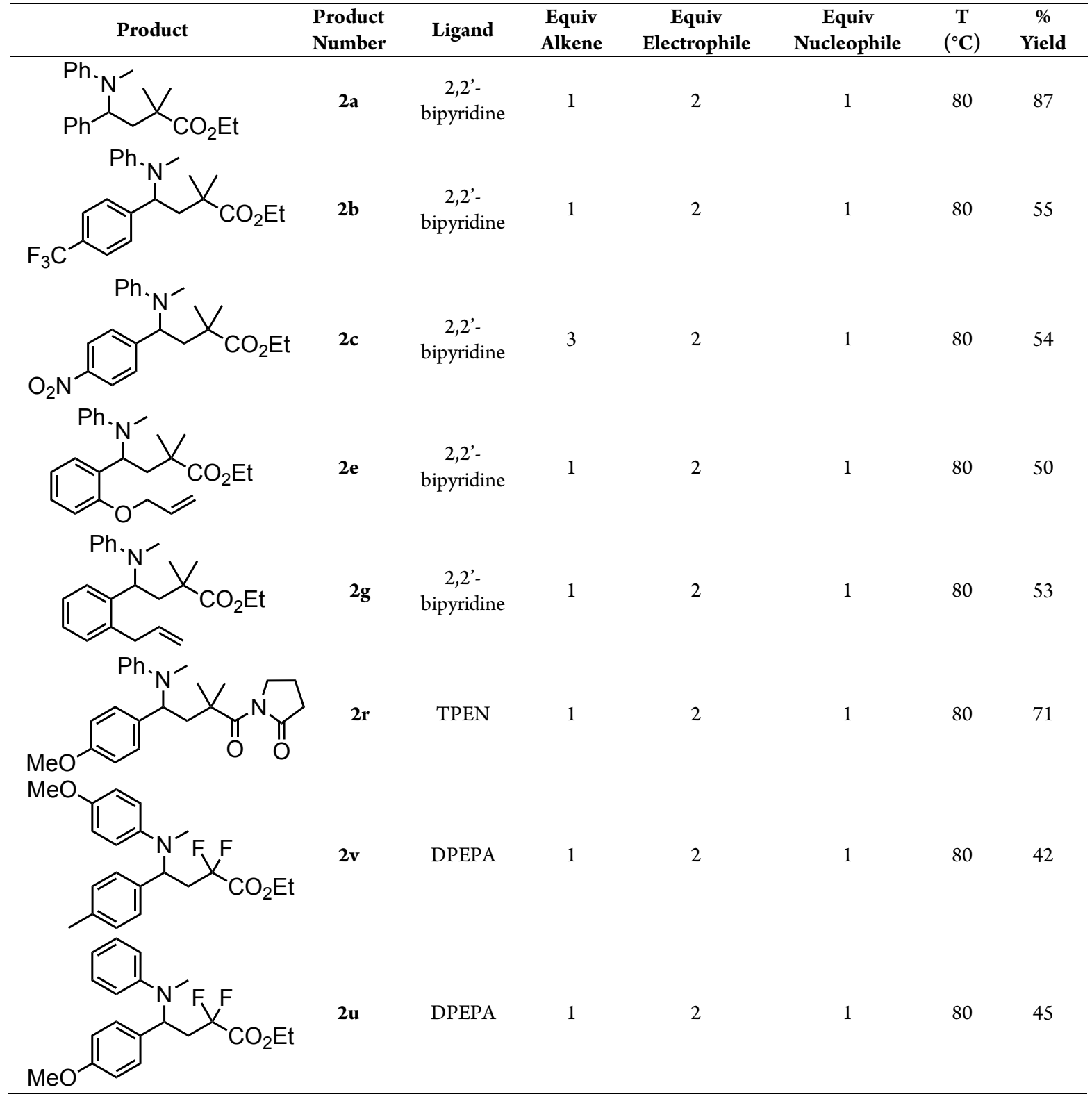




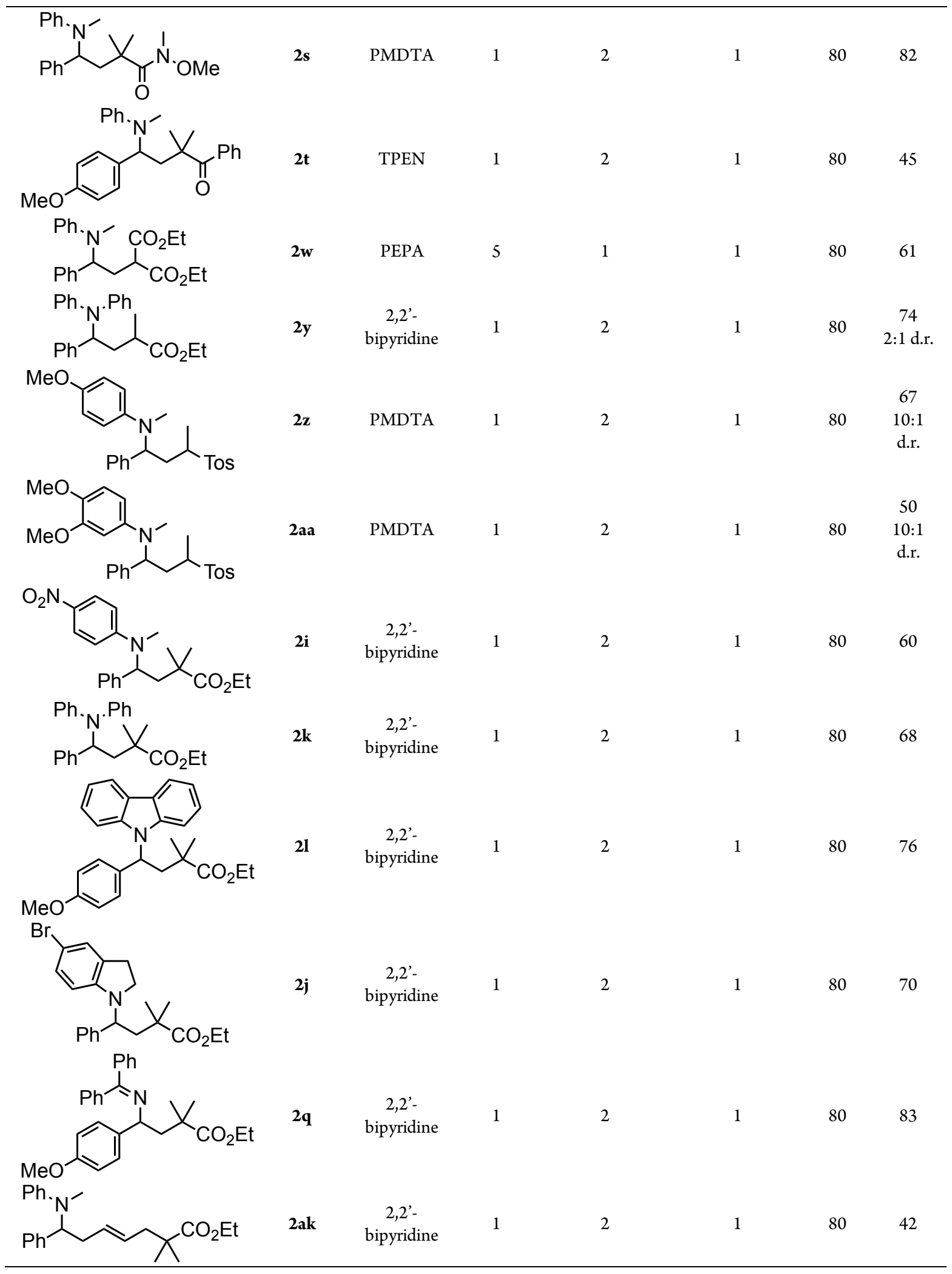




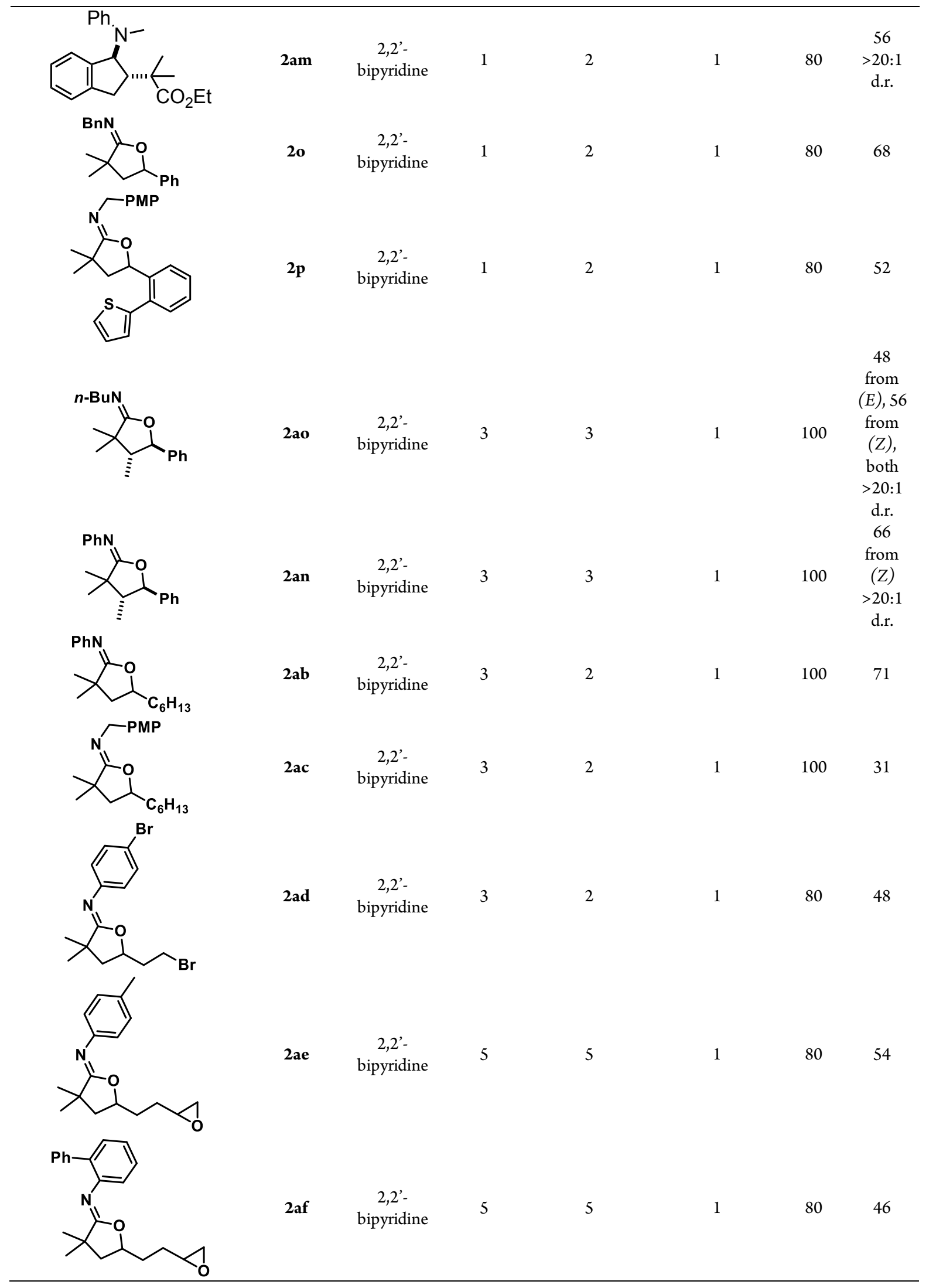




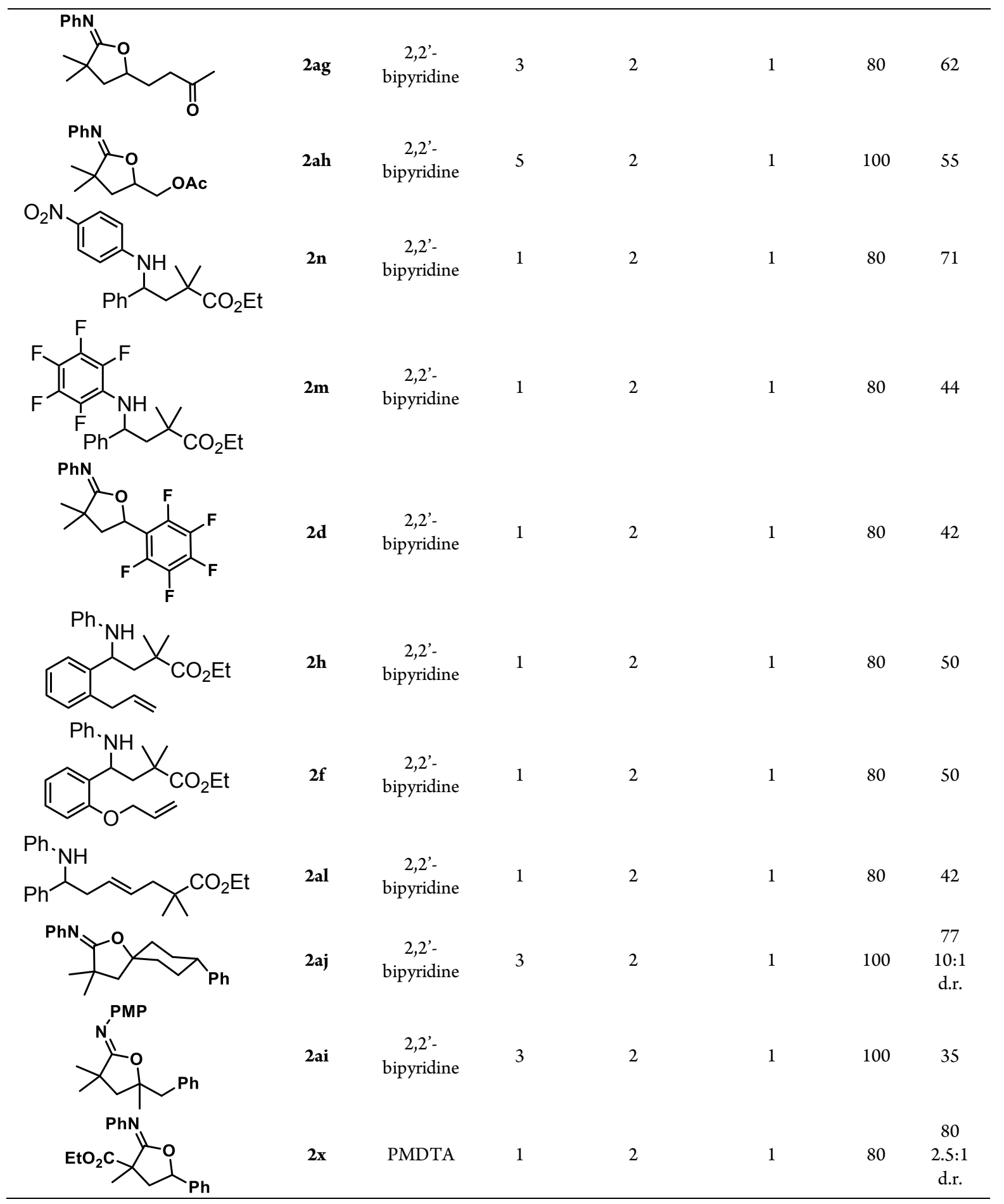


<smiles>c1ccc(CCN(CCc2ccccn2)Cc2ccccn2)nc1</smiles>

DPEPA<smiles>c1ccc(CN(CCN(Cc2ccccn2)Cc2ccccn2)Cc2ccccn2)nc1</smiles><smiles>CN(C)CCN(C)CCN(C)C</smiles>

PMDTA<smiles>c1ccc(CCNCc2ccccn2)nc1</smiles>

\section{Materials Preparation}

The materials preparation section is broken down into the following subsections:

DI. Alkene Syntheses

DII. Electrophile Syntheses

DIII. Nucleophile Syntheses

DIV. Ligand Syntheses

\section{Alkene Syntheses}

\section{Preparation of 2-(2-vinylphenyl)thiophene}

This substrate was prepared via this two-step route:

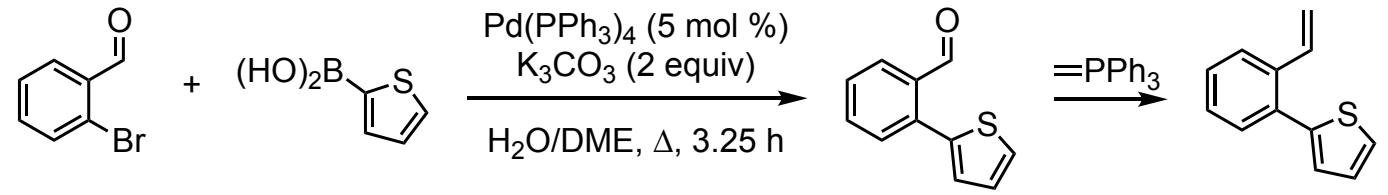

Step 1: Suzuki-Miyaura coupling to form 2-(thiophen-2-yl)benzaldehyde

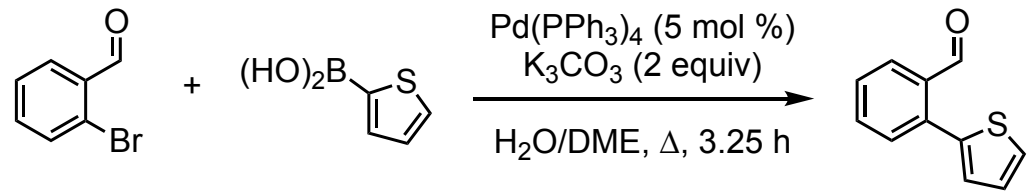

Prepared according to the patent literature: Bernardon, J.; Nedoncelle, P. Triaromatic compounds, compositions containing them and uses thereof. Eur. Pat. Appl. EP19980401144, 1998

A 100-mL 3-neck round bottom flask equipped with a stir bar and reflux condenser was charged with thiophen-2-ylboronic acid (1.92 g, $15.0 \mathrm{mmol}, 1.50$ equiv), 2-bromobenzaldehyde ( $1.17 \mathrm{~mL}, 10.0 \mathrm{mmol}$, 1.00 equiv), 1,2-dimethoxyethane $(40 \mathrm{~mL})$, and an aqueous solution of potassium carbonate $(2.76 \mathrm{~g}$ $\mathrm{K}_{2} \mathrm{CO}_{3}, 20 \mathrm{mmol}, 2.00$ equiv in $10 \mathrm{~mL} \mathrm{H}_{2} \mathrm{O}$ ). The resultant solution was sparged with a strong stream of nitrogen for 10 minutes, after which time tetrakis(triphenylphosphine)palladium(0) (577.8 mg, 0.500 $\mathrm{mmol}, 5.00 \mathrm{~mol} \%)$ was quickly added to the vessel. The reaction mixture was then refluxed for 3.25 hours. The mixture was diluted with deionized $\mathrm{H}_{2} \mathrm{O}$, and the organic materials extracted three times with ethyl acetate. The combined organic phases were dried with anhydrous magnesium sulfate, filtered, and concentrated by rotary evaporation. Flash column chromatography ( $5 \%$ ethyl acetate in hexanes) yielded the title compound as a pale-yellow oil in quantitative yield $(1.89 \mathrm{~g})$. 
${ }^{1} \mathrm{H} \mathrm{NMR}\left(500 \mathrm{MHz}, \mathrm{CDCl}_{3}\right) \delta 10.19(\mathrm{~d}, J=0.9 \mathrm{~Hz}, 1 \mathrm{H}), 8.01(\mathrm{dd}, J=7.9,1.4 \mathrm{~Hz}, 1 \mathrm{H}), 7.62(\mathrm{td}, J=7.5$, $1.4 \mathrm{~Hz}, 1 \mathrm{H}), 7.55(\mathrm{dd}, J=7.7,1.2 \mathrm{~Hz}, 1 \mathrm{H}), 7.51-7.46(\mathrm{~m}, 3 \mathrm{H}), 7.15(\mathrm{dd}, J=5.1,3.5 \mathrm{~Hz}, 1 \mathrm{H}), 7.08(\mathrm{dd}$, $J=3.6,1.2 \mathrm{~Hz}, 1 \mathrm{H})$

\section{Step 2: Olefination to form 2-(2-vinylphenyl)thiophene}<smiles>C=Cc1ccc(CC(C)C)cc1-c1cccs1</smiles>

Prepared according to the literature: Angew. Chem. Int. Ed. 2015, 54,12612

An oven dried 300-mL round bottom flask equipped with a stir bar was charged with methyltriphenylphosphonium bromide $(3.57 \mathrm{~g}, 10.0 \mathrm{mmol}, 1.00$ equiv). The atmosphere over the bromide salt was evacuated and refilled with nitrogen three times, then dry tetrahydrofuran $(85 \mathrm{~mL})$ was added to the flask. The mixture was cooled to $0^{\circ} \mathrm{C}$ in an ice bath, and then $n$-butyllithium (1.6 M in hexanes, $6.25 \mathrm{~mL}, 10 \mathrm{mmol}, 1.00$ equiv) was added dropwise. The resultant yellow solution was brought out of the ice bath and allowed to stir for 15 minutes, then 2-(thiophen-2-yl)benzaldehyde was added to the flask in one portion. The reaction was stirred for 1.5 hours, then was quenched with saturated ammonium chloride solution. The organic materials were extracted with dichloromethane three times, then the combined organic phases were dried with anhydrous magnesium sulfate, filtered, and concentrated by rotary evaporation. The crude material was re-dissolved in dichloromethane, adsorbed onto diatomaceous earth $\left(\mathrm{Celite}^{\odot}\right)$, and purified by flash column chromatography (100\% hexanes) to yield the title compound as a colorless oil ( $1.56 \mathrm{~g}, 84 \%)$.

${ }^{1} \mathrm{H} \mathrm{NMR}\left(400 \mathrm{MHz}, \mathrm{CDCl}_{3}\right): \delta(\mathrm{ppm}) 7.62(\mathrm{dd}, J=7.4,1.8 \mathrm{~Hz}, 1 \mathrm{H}), 7.43(\mathrm{dd}, J=7.2,1.9 \mathrm{~Hz}, 1 \mathrm{H}), 7.39$ $-7.28(\mathrm{~m}, 3 \mathrm{H}), 7.13-7.06(\mathrm{~m}, 2 \mathrm{H}), 6.98(\mathrm{dd}, J=17.4,11.0 \mathrm{~Hz}, 1 \mathrm{H}), 5.72(\mathrm{dd}, J=17.5,1.3 \mathrm{~Hz}, 1 \mathrm{H})$, $5.27(\mathrm{dd}, J=11.0,1.4 \mathrm{~Hz}, 1 \mathrm{H})$

${ }^{13} \mathrm{C} \mathrm{NMR}\left(126 \mathrm{MHz}, \mathrm{CDCl}_{3}\right):(\mathrm{ppm}) \delta 142.41,136.87,136.24,133.42,130.85,128.22,127.93,127.90$, $127.47,126.64,125.92,115.69$

\section{Preparation of $( \pm)$-trans-(2-vinylcyclopropyl)benzene}

The cyclopropyl substrate was prepared via this three-step route:

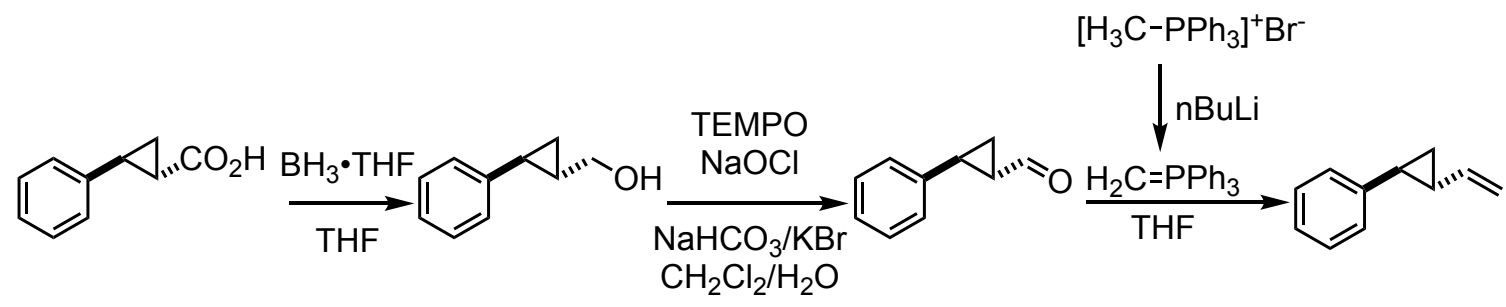

\section{Step 1: Reduction

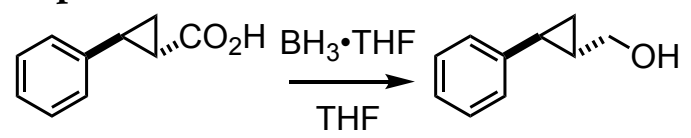

An oven-dried 100-mL three-necked flask equipped with a stir bar was charged with ( \pm )-trans-2phenylcyclopropane-1-carboxylic acid $(1.64 \mathrm{~g}, 10.1 \mathrm{mmol})$. Anhydrous THF $(18 \mathrm{~mL}, 0.56 \mathrm{M})$ was added and the suspension was cooled to $0{ }^{\circ} \mathrm{C} . \mathrm{BH}_{3}$. THF (1.0 M solution in THF, $12.2 \mathrm{~mL}, 12.2 \mathrm{mmol}, 1.21$ 
equiv) was added very slowly in a dropwise fashion. The mixture was stirred at $0{ }^{\circ} \mathrm{C}$ for $1 \mathrm{~h}$, after which point it was stirred at room temperature for $1 \mathrm{~h}$. GC-MS analysis indicated starting material remained and additional $\mathrm{BH}_{3} \cdot \mathrm{THF}(4.42 \mathrm{~mL}, 4.42 \mathrm{mmol}, 0.440$ equiv) was thus added at room temperature and the mixture was allowed to stir an additional $1.25 \mathrm{~h}$. The reaction was then quenched at $0{ }^{\circ} \mathrm{C}$ with the addition of $\mathrm{MeOH}$ in a slow, dropwise fashion. Deionized water was next added until two layers form. The mixture was transferred to a separatory funnel with ethyl acetate. Brine was added and the aqueous layer was washed $3 \times 25 \mathrm{~mL}$ with ethyl acetate. The combined organic layers were washed $2 \times 20 \mathrm{~mL}$ with brine. The combined organic layers were then dried with anhydrous sodium sulfate, filtered, and the solvent was removed under reduced pressure with the aid of a rotary evaporator to provide the crude product $(1.48 \mathrm{~g})$. The crude product was used directly in the next step without further purification.

\section{Step 2: Oxidation}

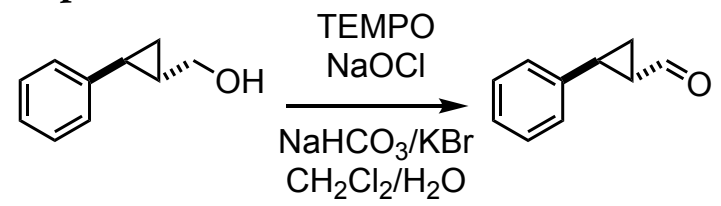

A 250-mL round-bottomed flask equipped with a stir bar was charged with crude ( \pm )-trans-2phenylcyclopropyl)methanol from the previous step $(1.48 \mathrm{~g}, 10 \mathrm{mmol})$ and methylene chloride $(25 \mathrm{~mL}$, $0.40 \mathrm{M})$. The mixture was cooled to $0{ }^{\circ} \mathrm{C}$ and TEMPO $(16 \mathrm{mg}, 1.0 \mathrm{~mol} \%)$ was added. An aqueous solution of $\mathrm{KBr}$ and $\mathrm{NaHCO}_{3}\left(1.10\right.$ equiv $\mathrm{KBr}$, 3.30 equiv $\mathrm{NaHCO}_{3}$ in $56 \mathrm{~mL} \mathrm{H}_{2} \mathrm{O}$ ) was added and stirring was engaged. Commercial bleach $(13 \mathrm{~mL})$ was next added and the dark red biphasic mixture was stirred 5 min. GC-MS indicated a small amount of starting material remained and an additional $4 \mathrm{~mL}$ bleach was added. The mixture was stirred for $1 \mathrm{~h}$ at $0{ }^{\circ} \mathrm{C}$ and then quenched with saturated sodium thiosulfate. The layers were separated with the aid of a separatory funnel and the aqueous layer was washed $3 \times 25 \mathrm{~mL}$ with methylene chloride. The combined organic layers were dried with anhydrous magnesium sulfate, filtered, and the solvent was removed under reduced pressure with a rotary evaporator to afford the crude product $(1.60 \mathrm{~g})$, which was used in the next step without further purification.

\section{Step 3: Olefination}

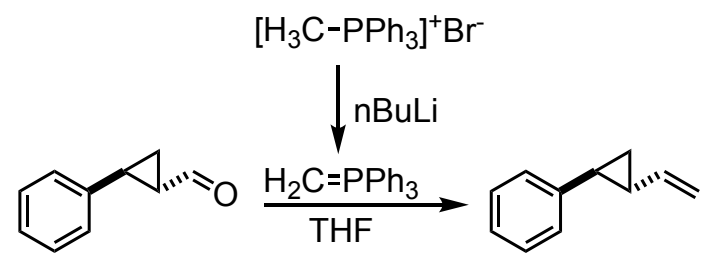

A 250-mL oven-dried round-bottomed flask equipped with a stir bar was charged with methyltriphenylphosphonium bromide $(2.51 \mathrm{~g}, 7.04 \mathrm{mmol})$. The flask was placed under an atmosphere of nitrogen using standard Schlenk technique and anhydrous THF $(50 \mathrm{~mL}, 0.14 \mathrm{M})$ was added through the septum. The suspension was cooled to $0{ }^{\circ} \mathrm{C}$ with stirring and $\mathrm{n}$-butyllithium ( $1.60 \mathrm{M}$ in hexanes, 4.40 $\mathrm{mL}, 7.04 \mathrm{mmol}, 1.00$ equiv) was added in a dropwise fashion. The cooling bath was removed and the mixture was stirred at room temperature for $30 \mathrm{~min}$. Next, a solution of crude ( \pm )-trans-2phenylcyclopropane-1-carbaldehyde $(1.03 \mathrm{~g}$, $7.04 \mathrm{mmol}, 1.00$ equiv $)$ in THF $(6 \mathrm{~mL})$ was added through the septum at room temperature. The mixture was stirred at room temperature until the reaction was complete as indicated by TLC (product $\mathrm{R}_{f}=0.9$ in $5 \%$ ethyl acetate in hexanes). The mixture was quenched at room temperature with saturated aqueous ammonium chloride. The aqueous layer was washed with methylene chloride $(3 \times 50 \mathrm{~mL})$. The combined organic layers are dried with anhydrous sodium sulfate, filtered, and the solvent was removed under reduced pressure with the aid of a rotary 
evaporator. The crude product was purified via flash column chromatography on silica (100\% hexanes) to provide pure $( \pm$ )-trans-(2-vinylcyclopropyl)benzene ( $0.68 \mathrm{~g}, 67 \%$ yield). The spectral data matched those previously reported. (Bull. Korean Chem. Soc. 2006, 27, 765-768.)

${ }^{1} \mathrm{H} \mathrm{NMR}\left(500 \mathrm{MHz}, \mathrm{CDCl}_{3}\right): \delta(\mathrm{ppm}) 7.30-7.23(\mathrm{~m}, 2 \mathrm{H}), 7.16(\mathrm{t}, J=7.4 \mathrm{~Hz}, 1 \mathrm{H}), 7.08(\mathrm{~d}, J=6.9 \mathrm{~Hz}$, $2 \mathrm{H}), 5.54(\mathrm{ddd}, J=17.1,10.3,8.5 \mathrm{~Hz}, 1 \mathrm{H}), 5.11(\mathrm{ddd}, J=17.1,1.6,0.7 \mathrm{~Hz}, 1 \mathrm{H}), 4.94(\mathrm{dd}, J=10.3,1.5$ $\mathrm{Hz}, 1 \mathrm{H}), 1.93(\mathrm{ddd}, J=8.8,5.7,4.3 \mathrm{~Hz}, 1 \mathrm{H}), 1.71$ (ddd, $J=8.5,5.7,4.2 \mathrm{~Hz}, 1 \mathrm{H}), 1.21$ (ddd, $J=8.5,5.7$, $5.0 \mathrm{~Hz}, 1 \mathrm{H}), 1.11(\mathrm{ddd}, J=8.8,5.3,5.3 \mathrm{~Hz}, 1 \mathrm{H})$

${ }^{13} \mathrm{C} \mathrm{NMR}\left(125 \mathrm{MHz}, \mathrm{CDCl}_{3}\right): \delta(\mathrm{ppm}) 142.61,140.93,128.61,125.96,125.90,112.81,27.68,25.52$, 17.02

\section{Synthesis of (4-methylenecyclohexyl)benzene:}

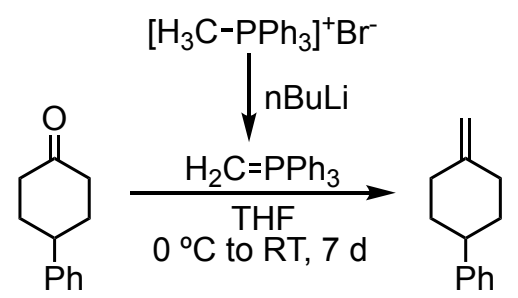

A 250-mL oven-dried round-bottomed flask equipped with a stir bar was charged with methyltriphenylphosphonium bromide $(2.51 \mathrm{~g}, 7.04 \mathrm{mmol})$. The flask was placed under an atmosphere of nitrogen using standard Schlenk technique and anhydrous THF (58 mL, $0.12 \mathrm{M}$ ) was added through the septum. The suspension was cooled to $0{ }^{\circ} \mathrm{C}$ with stirring and n-butyllithium ( $1.60 \mathrm{M}$ in hexanes, 4.41 $\mathrm{mL}, 7.06 \mathrm{mmol}, 1.00$ equiv) was added in a dropwise fashion. The cooling bath was removed and the mixture was stirred at room temperature for $30 \mathrm{~min}$. Next, a solution of 4-phenylcyclohexanone $(1.23 \mathrm{~g}$, $7.06 \mathrm{mmol}, 1.00$ equiv) in THF $(10 \mathrm{~mL})$ was added through the septum at room temperature. The mixture was stirred at room temperature for $7 \mathrm{~d}$. The mixture was then quenched at room temperature with saturated aqueous ammonium chloride. The aqueous layer was washed with methylene chloride $(3 \times 50 \mathrm{~mL})$. The combined organic layers are dried with anhydrous sodium sulfate, filtered, and the solvent was removed under reduced pressure with the aid of a rotary evaporator. The crude product was purified via flash column chromatography on silica (0-2.5\% ethyl acetate in hexanes) to provide pure (4methylenecyclohexyl)benzene $(0.57 \mathrm{~g}, 47 \%$ yield $)$. The spectral data matched those previously reported. (Org. Lett. 2017, 19, 3374-3377.)

${ }^{1} \mathrm{H} \mathrm{NMR}\left(500 \mathrm{MHz}, \mathrm{CDCl}_{3}\right): \delta(\mathrm{ppm}) 7.30(\mathrm{t}, J=7.6 \mathrm{~Hz}, 2 \mathrm{H}), 7.24-7.16(\mathrm{~m}, 3 \mathrm{H}), 4.69(\mathrm{t}, J=1.8 \mathrm{~Hz}$, $2 \mathrm{H}), 2.68(\mathrm{tt}, J=12.3,3.4 \mathrm{~Hz}, 1 \mathrm{H}), 2.43(\mathrm{ddt}, J=13.5,4.0,1.9 \mathrm{~Hz}, 2 \mathrm{H}), 2.20(\mathrm{tt}, J=14.3,12.6,4.0,1.8$ $\mathrm{Hz}, 2 \mathrm{H}), 1.99$ (dp, $J=12.7,3.6,1.9 \mathrm{~Hz}, 2 \mathrm{H}), 1.56(\mathrm{qd}, J=12.8,4.0 \mathrm{~Hz}, 2 \mathrm{H})$

${ }^{13} \mathrm{C} \mathrm{NMR}\left(125 \mathrm{MHz}, \mathrm{CDCl}_{3}\right): \delta(\mathrm{ppm}) 149.14,147.16,128.64,127.13,126.28,107.64,44.45,35.81$, 35.45

\section{Electrophile Syntheses}

\section{1-(2-bromo-2-methylpropanoyl)pyrrolidin-2-one}

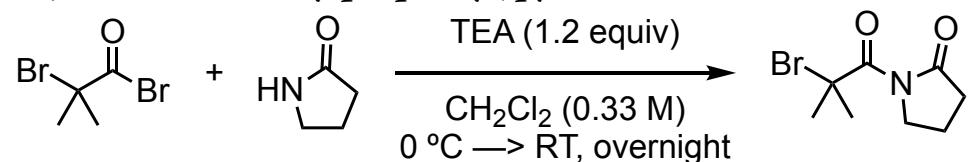

Prepared according to the literature: Org. Lett. 2014, 16, 3166 
An oven dried $100 \mathrm{~mL}$ round bottom flask equipped with a stir bar and under nitrogen was charged with 2-pyrrolidone ( $12.0 \mathrm{mmol}, 0.912 \mathrm{~mL}, 1.20$ equiv), dichloromethane $(33.0 \mathrm{~mL})$, and triethylamine $(12.0$ mmol, $1.67 \mathrm{~mL}, 1.20$ equiv). The resultant solution was cooled in an ice bath whilst stirring, after which time 2-bromoisobutyryl bromide ( $10 \mathrm{mmol}, 1.24 \mathrm{~mL}, 1.00$ equiv) was added dropwise. The flask was brought out of the ice bath and stirred at ambient temperature overnight. The contents of the flask were diluted with deionized water, then transferred to a separatory funnel with dichloromethane. After collecting the organic phase, the aqueous solution was extracted three times with dichloromethane, then the combined organic phase was dried with sodium sulfate. Flash column chromatography using $20 \%$ ethyl acetate in hexanes as eluent yielded the pure title compound as an amber oil (1.45 g, 62\%).

${ }^{1} \mathrm{H} \mathrm{NMR}\left(500 \mathrm{MHz}, \mathrm{CDCl}_{3}\right): \delta(\mathrm{ppm}) 3.90-3.87(\mathrm{~m}, 1 \mathrm{H}), 2.64(\mathrm{t}, J=8.1 \mathrm{~Hz}, 2 \mathrm{H}), 2.10-2.05(\mathrm{~m}$, $2 \mathrm{H}), 2.04(\mathrm{~s}, 6 \mathrm{H})$

${ }^{13} \mathrm{C}$ NMR $\left(126 \mathrm{MHz}, \mathrm{CDCl}_{3}\right): \delta(\mathrm{ppm}) 173.23,172.52,58.07,48.78,34.24,30.98,17.96$

\section{Nucleophile Syntheses}

\section{3,4-dimethoxy- $\mathrm{N}$-methylaniline Synthesis:}

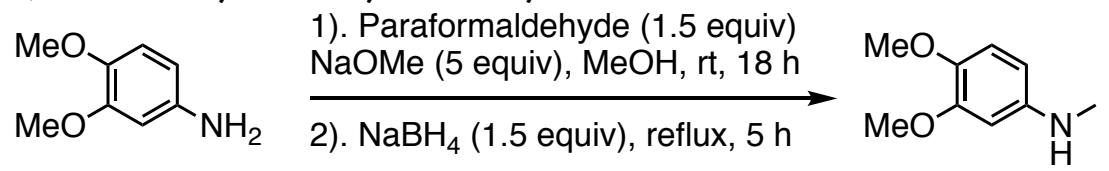

A 50-mL round-bottomed flask equipped with a stir bar was charged with 3,4-dimethoxy- $\mathrm{N}$ methylaniline (1.53 g, $10.0 \mathrm{mmol}, 1.00$ equiv), paraformaldehyde $(0.53 \mathrm{~g}, 15.0 \mathrm{mmol}, 1.50$ equiv), and methanol $(20 \mathrm{~mL}, 0.50 \mathrm{M})$. The brown mixture was stirred rapidly and sodium methoxide $(2.82 \mathrm{~g}, 52.0$ mmol, 5.20 equiv) was added over $2 \mathrm{~min}$. A reflux condenser and nitrogen inlet were attached and the mixture was stirred at room temperature $20 \mathrm{~h} . \mathrm{NaBH}_{4}(0.51 \mathrm{~g}, 13.4 \mathrm{mmol}, 1.34$ equiv) was added and the mixture was heated at $80^{\circ} \mathrm{C}$ for $5.5 \mathrm{~h}$. The mixture was cooled and quenched with saturated aqueous sodium bicarbonate. The biphasic mixture was transferred to a separatory funnel with deionized water and ethyl acetate. The organic layer was separated and the aqueous layer was washed $3 \times 20 \mathrm{~mL}$ with ethyl acetate. The combined organic layers were dried with anhydrous sodium sulfate, filtered, and the solvent was removed with the aid of a rotary evaporator to afford a crude dark maroon oil. The product was purified via flash column chromatography on silica (30-40\% ethyl acetate in hexanes) to afford the title compound as a pale yellow oil in a quantitative yield. Spectral data matched those previously reported (Science 2016, 353, 1144-1147.).

${ }^{1} \mathrm{H} \operatorname{NMR}\left(500 \mathrm{MHz}, \mathrm{CDCl}_{3}\right): \delta(\mathrm{ppm}) 6.77(\mathrm{~d}, J=8.5 \mathrm{~Hz}, 1 \mathrm{H}), 6.25(\mathrm{~d}, J=2.7 \mathrm{~Hz}, 1 \mathrm{H}), 6.15(\mathrm{dd}, J=$ $8.5,2.6 \mathrm{~Hz}, 1 \mathrm{H}), 3.85(\mathrm{~s}, 3 \mathrm{H}), 3.81(\mathrm{~s}, 3 \mathrm{H}), 2.81(\mathrm{~s}, 3 \mathrm{H})$

${ }^{13} \mathrm{C} \mathrm{NMR}\left(125 \mathrm{MHz}, \mathrm{CDCl}_{3}\right): \delta(\mathrm{ppm}) 150.32,144.64,141.80,113.62,103.30,98.83,57.06,56.01$, 31.78

\section{Ligand Syntheses}

PEPA and DPEPA can be synthesized through this procedure. An alternative, higher yielding synthesis of PEPA is on the next page. 


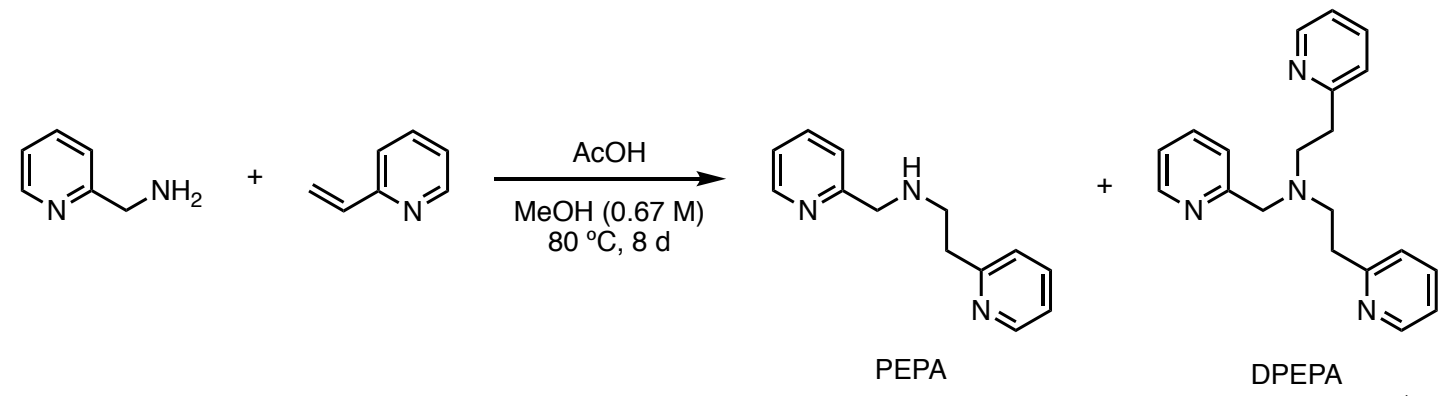

A 50-mL round-bottomed flask equipped with a stir bar was charged with anhydrous methanol $(12 \mathrm{~mL}$, $0.67 \mathrm{M})$, pyridin-2-ylmethanamine $(0.82 \mathrm{~mL}, 8.0 \mathrm{mmol}, 1.0$ equiv), 2-vinylpyridine $(3.0 \mathrm{~mL}, 24 \mathrm{mmol}$, 3.0 equiv), and acetic acid (glacial, $1.3 \mathrm{~mL}$ ). A reflux condenser was installed, the mixture was placed under a nitrogen atmosphere, and stirring was engaged. The temperature was slowly raised to $80^{\circ} \mathrm{C}$ and the mixture was stirred at that temperature for $8 \mathrm{~d}$. The reaction was then cooled and $20 \mathrm{~mL}$ of $5 \mathrm{M}$ $\mathrm{NaOH}$ was added and the mixture was stirred for $2 \mathrm{~h}$ at room temperature. It was then transferred to separatory funnel and washed with methylene chloride $(3 \times 25 \mathrm{~mL})$. The combined organic layer was washed once with water. It was then dried with anhydrous sodium sulfate, filtered, and the solvent was removed under reduced pressure with the aid of a rotavap. The black oil was purified by column chromatography on basic alumina deactivated with water, eluting with $10 \%$ ethyl acetate in hexanes $+1 \%$ methanol and ramping up to $60 \%$ ethyl acetate in hexanes $+3 \%$ methanol. The two products, PEPA and DPEPA were cleanly separated and isolated. PEPA ( $139.9 \mathrm{mg}, 8 \%$ yield $)$ and DPEPA ( $1.65 \mathrm{~g}, 65 \%$ yield $)$ were obtained as yellow oils.

PEPA:

${ }^{1} \mathrm{H}$ NMR $\left(500 \mathrm{MHz}, \mathrm{CDCl}_{3}\right): \delta(\mathrm{ppm}) 8.56-8.51(\mathrm{~m}, 2 \mathrm{H}), 7.60$ (dddd, $\left.J=15.3,7.7,1.9 \mathrm{~Hz}, 2 \mathrm{H}\right), 7.31-$ $7.28(\mathrm{~m}, 1 \mathrm{H}), 7.18(\mathrm{dt}, J=7.9,1.0 \mathrm{~Hz}, 1 \mathrm{H}), 7.12$ (dddd, $J=12.7,7.6,4.9,1.2 \mathrm{~Hz}, 2 \mathrm{H}), 3.95(\mathrm{~s}, 2 \mathrm{H}), 3.10$ $3.05(\mathrm{~m}, 2 \mathrm{H}), 3.05-3.00(\mathrm{~m}, 2 \mathrm{H})$.

${ }^{13} \mathrm{C}$ NMR $\left(125 \mathrm{MHz}, \mathrm{CDCl}_{3}\right): \delta(\mathrm{ppm}) 160.51,160.13,149.63,149.56,136.67,136.59,123.55,122.46$, $122.13,121.50,55.47,49.39,38.92$.

\section{DPEPA:}

${ }^{1} \mathrm{H}$ NMR $\left(500 \mathrm{MHz}, \mathrm{CDCl}_{3}\right): \delta(\mathrm{ppm}) 8.48(\mathrm{ddd}, J=4.9,1.9,1.0 \mathrm{~Hz}, 3 \mathrm{H}), 7.52(\mathrm{tt}, J=7.7,1.4 \mathrm{~Hz}, 2 \mathrm{H})$, $7.48(\mathrm{tt}, J=7.7,1.4 \mathrm{~Hz}, 1 \mathrm{H}), 7.11(\mathrm{~d}, J=7.8 \mathrm{~Hz}, 2 \mathrm{H}), 7.08$ (dddd, $J=7.4,4.6,2.6,1.3 \mathrm{~Hz}, 2 \mathrm{H}), 7.04$ (dd, $J$ $=7.9,1.2 \mathrm{~Hz}, 2 \mathrm{H}), 3.87(\mathrm{~s}, 2 \mathrm{H}), 3.00(\mathrm{~m}, 4 \mathrm{H}), 2.95(\mathrm{~m}, 4 \mathrm{H})$.

${ }^{13} \mathrm{C} \operatorname{NMR}\left(125 \mathrm{MHz}, \mathrm{CDCl}_{3}\right): \delta(\mathrm{ppm}) 160.92,160.54,149.45,149.09,136.47,136.31,123.66,122.89$, $121.96,121.26,60.69,54.54,36.38$.

\section{Synthesis of PEPA}

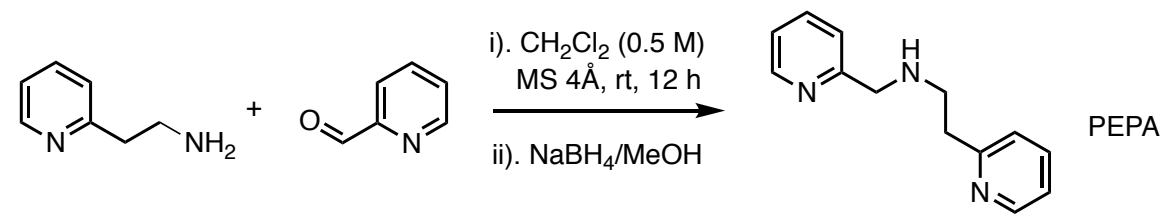

An oven-dried 50-mL round-bottomed flask equipped with a stir bar was charged with oven-dried MS $4 \AA$ and dry methylene chloride $(10 \mathrm{~mL}, 0.5 \mathrm{M})$. 2-(pyridin-2-yl)ethan-1-amine $(0.60 \mathrm{~mL}, 5.0 \mathrm{mmol})$ and picolinaldehyde $(0.48 \mathrm{~mL}, 5.0 \mathrm{mmol})$ were successively added. The flask was sealed with a septum and stirred overnight at room temperature. The mixture was then filtered over diatomaceous earth (Celite $\left.{ }^{\circledast}\right)$ and the solvent was removed under reduced pressure with the aid of a rotavap to afford the crude imine. 
Anhydrous methanol $(8 \mathrm{~mL})$ was then added and the solution was cooled to $0{ }^{\circ} \mathrm{C}$ before $\mathrm{NaBH}_{4}(0.27 \mathrm{~g}$, 0.89 equiv) was slowly added. The mixture was stirred under a nitrogen atmosphere for $5 \mathrm{~h}$, after which point the reaction was quenched with saturated aqueous $\mathrm{K}_{2} \mathrm{CO}_{3}$. The mixture was transferred to a separatory funnel and washed with methylene chloride $(3 \times 10 \mathrm{~mL})$. The combined organic layers were dried with anhydrous magnesium sulfate, filtered over diatomaceous earth $\left(\mathrm{Celite}^{\circledast}\right)$, and the solvent was removed under reduced pressure with the aid of a rotary evaporator to afford PEPA as a pale yellow oil in a quantitative yield. The product was pure as deemed by ${ }^{1} \mathrm{H}$ NMR analysis and no further purification was necessary. The spectral data matched that reported above.

\section{E. Experimental Procedure, Isolation, and Characterization}


Ethyl 2,2-dimethyl-4-(methyl(phenyl)amino)-4-phenylbutanoate (2a)<smiles>CCOC(C)(C)CC(c1ccccc1)N(C)c1ccccc1</smiles>

In a nitrogen-filled glove box, an oven-dried 4-mL reaction vial was charged with $\mathrm{Cu}(\mathrm{OTf})_{2}(3.6 \mathrm{mg}, 10$ $\mu \mathrm{mol}, 5.0 \mathrm{~mol} \%), \mathrm{K}_{3} \mathrm{PO}_{4}(46.6 \mathrm{mg}, 0.220 \mathrm{mmol}, 1.10$ equiv), and a stir bar. Next, $400 \mu \mathrm{L}$ of a DCE stock solution containing 2,2'-bipyridine (0.025 M, $10 \mu \mathrm{mol}, 5.0 \mathrm{~mol} \%)$ was added. This was followed by addition of styrene $(22.9 \mu \mathrm{L}, 0.200 \mathrm{mmol}, 1.00$ equiv), $N$-methylaniline $(22.4 \mu \mathrm{L}, 0.200 \mathrm{mmol}, 1.00$ equiv), and ethyl 2-bromo-2-methylpropanoate $(58.7 \mu \mathrm{L}, 0.400 \mathrm{mmol}, 2.00$ equiv). The vial was sealed with a Teflon-lined cap, removed from the glove box, and heated at $80^{\circ} \mathrm{C}$ with stirring at $660 \mathrm{rpm}$ for 24 $\mathrm{h}$. The crude mixture was then directly adsorbed onto diatomaceous earth (Celite ${ }^{\circledast}$ ) and purified by flash column chromatography ( $2.5 \%$ ethyl acetate in hexanes). The product was obtained as a colorless oil (56.5 mg, 87\%).

$\mathrm{R}_{f}=0.26$ (5\% ethyl acetate in hexanes)

${ }^{1} \mathrm{H} \mathrm{NMR}\left(500 \mathrm{MHz}, \mathrm{CDCl}_{3}\right): \delta(\mathrm{ppm}) 7.22(\mathrm{~m}, 7 \mathrm{H}), 6.81(\mathrm{~d}, J=8.0 \mathrm{~Hz}, 2 \mathrm{H}), 6.72(\mathrm{tt}, J=7.2,0.9 \mathrm{~Hz}$, $1 \mathrm{H}), 5.13(\mathrm{dd}, J=9.9,3.7 \mathrm{~Hz}, 1 \mathrm{H}), 3.86(\mathrm{dq}, J=10.8,7.1 \mathrm{~Hz}, 1 \mathrm{H}), 3.80(\mathrm{dq}, J=10.8,7.2 \mathrm{~Hz}, 1 \mathrm{H}), 2.57$ $(\mathrm{s}, 3 \mathrm{H}), 2.52(\mathrm{dd}, J=14.4,10.0 \mathrm{~Hz}, 1 \mathrm{H}), 2.11(\mathrm{dd}, J=14.4,3.7 \mathrm{~Hz}, 1 \mathrm{H}), 1.29(\mathrm{~s}, 3 \mathrm{H}), 1.27(\mathrm{~s}, 3 \mathrm{H}), 1.03$ $(\mathrm{t}, J=7.1 \mathrm{~Hz}, 3 \mathrm{H})$

${ }^{13} \mathrm{C}$ NMR $\left(125 \mathrm{MHz}, \mathrm{CDCl}_{3}\right): \delta(\mathrm{ppm}) 177.41,150.43,141.11,129.34,128.51,127.38,127.27,117.28$, $114.15,60.78,58.62,42.48,41.54,32.76,27.31,25.40,14.15$

HRMS (ESI-TOF) $m / z:\left[\mathrm{M}+\mathrm{H}^{+}\right]$calculated for $\mathrm{C}_{21} \mathrm{H}_{28} \mathrm{NO}_{2}, 326.2120$; found, 326.2128

Ethyl 2,2-dimethyl-4-(methyl(phenyl)amino)-4-(4-(trifluoromethyl)phenyl)butanoate (2b)<smiles>CCOC(C)(C)CC(c1ccc(C(F)(F)F)cc1)N(C)c1ccccc1</smiles>

In a nitrogen-filled glove box, an oven-dried 4-mL reaction vial was charged with $\mathrm{Cu}(\mathrm{OTf})_{2}(3.6 \mathrm{mg}, 10$ $\mu \mathrm{mol}, 5.0 \mathrm{~mol} \%), \mathrm{K}_{3} \mathrm{PO}_{4}(46.6 \mathrm{mg}, 0.22 \mathrm{mmol}, 1.10$ equiv), and a stir bar. Next, $400 \mu \mathrm{L}$ of a DCE stock solution containing 2,2'-bipyridine $(0.025 \mathrm{M}, 10 \mu \mathrm{mol}, 5.0 \mathrm{~mol} \%)$ was added. This was followed by addition of 4-trifluoromethylstyrene $(29.6 \mu \mathrm{L}, 0.200 \mathrm{mmol}, 1.00$ equiv), $N$-methylaniline $(21.7 \mu \mathrm{L}, 0.200$ mmol, 1.00 equiv), and ethyl 2-bromo-2-methylpropanoate ( $58.7 \mu \mathrm{L}, 0.400 \mathrm{mmol}, 2.00$ equiv). The vial was sealed with a Teflon-lined cap, removed from the glove box, and heated at $80^{\circ} \mathrm{C}$ with stirring at 660 rpm for $24 \mathrm{~h}$. The crude mixture was then directly adsorbed onto diatomaceous earth (Celite ${ }^{\circledast}$ ) and purified by flash column chromatography ( $5 \%$ ethyl acetate in hexanes). The product was obtained as a clear oil $(42.9 \mathrm{mg}, 55 \%)$.

$\mathrm{R}_{\mathrm{f}}=0.20$ (5\% ethyl acetate in hexanes)

${ }^{1} \mathrm{H} \mathrm{NMR}\left(500 \mathrm{MHz}, \mathrm{CDCl}_{3}\right): \delta(\mathrm{ppm}) 7.54(\mathrm{~d}, J=8.1 \mathrm{~Hz}, 2 \mathrm{H}), 7.33(\mathrm{~d}, J=8.0 \mathrm{~Hz}, 2 \mathrm{H}), 7.26(\mathrm{t}, J=8.0$ $\mathrm{Hz}, 2 \mathrm{H}), 6.83(\mathrm{~d}, J=8.3 \mathrm{~Hz}, 2 \mathrm{H}), 6.79(\mathrm{t}, J=7.3 \mathrm{~Hz}, 1 \mathrm{H}), 5.18(\mathrm{dd}, J=9.4,3.3 \mathrm{~Hz}, 1 \mathrm{H}), 3.96-3.80(\mathrm{~m}$, $2 \mathrm{H}), 2.62(\mathrm{~s}, 3 \mathrm{H}), 2.56(\mathrm{dd}, J=14.5,9.8 \mathrm{~Hz}, 1 \mathrm{H}), 2.15(\mathrm{dd}, J=14.4,3.7 \mathrm{~Hz}, 1 \mathrm{H}), 1.34(\mathrm{~s}, 3 \mathrm{H}), 1.31(\mathrm{~s}$, $3 \mathrm{H}), 1.08(\mathrm{t}, J=7.1 \mathrm{~Hz}, 3 \mathrm{H})$ 
${ }^{13} \mathrm{C}$ NMR (126 MHz, $\left.\mathrm{CDCl}_{3}\right): \delta(\mathrm{ppm}) 177.26,150.09,145.18,129.54\left(\mathrm{q}, J_{\mathrm{CF}}=32.8 \mathrm{~Hz}\right), 129.47$, $127.66,125.47\left(\mathrm{q}, J_{\mathrm{CF}}=3.8 \mathrm{~Hz}\right), 124.41\left(\mathrm{q}, J_{\mathrm{CF}}=272.2 \mathrm{~Hz}\right), 117.86,114.33,60.87,58.78,42.35,41.59$, $32.85,27.04,25.60,14.14$

${ }^{19} \mathrm{~F} \mathrm{NMR}\left(470 \mathrm{MHz}, \mathrm{CDCl}_{3}\right): \delta(\mathrm{ppm})-62.50$

HRMS (ESI-TOF) $m / z:\left[\mathrm{M}+\mathrm{H}^{+}\right]$calculated for $\mathrm{C}_{22} \mathrm{H}_{27} \mathrm{NO}_{2} \mathrm{~F}_{3}, 394.1994$; found, 394.1983

Ethyl 2,2-dimethyl-4-(methyl(phenyl)amino)-4-(4-nitrophenyl)butanoate (2c)<smiles>CCOC(=O)CC(C)(C)N(C)c1ccccc1</smiles>

In a nitrogen-filled glove box, an oven-dried $4-\mathrm{mL}$ reaction vial was charged with $\mathrm{Cu}(\mathrm{OTf})_{2}(3.6 \mathrm{mg}, 10$ $\mu \mathrm{mol}, 5.0 \mathrm{~mol} \%), \mathrm{K}_{3} \mathrm{PO}_{4}(46.6 \mathrm{mg}, 0.220 \mathrm{mmol}, 1.10$ equiv), and a stir bar. Next, $400 \mu \mathrm{L}$ of a DCE stock solution containing 2,2'-bipyridine $(0.025 \mathrm{M}, 10 \mu \mathrm{mol}, 5.0 \mathrm{~mol} \%)$ was added. This was followed by addition of 4-nitrostyrene $(76.9 \mu \mathrm{L}, 0.600 \mathrm{mmol}, 3.00$ equiv $), N$-methylaniline $(21.6 \mu \mathrm{L}, 0.200 \mathrm{mmol}$, 1.00 equiv), and ethyl 2-bromo-2-methylpropanoate ( $58.7 \mu \mathrm{L}, 0.400 \mathrm{mmol}, 2.00$ equiv). The vial was sealed with a Teflon-lined cap, removed from the glove box, and heated at $80^{\circ} \mathrm{C}$ with stirring at $660 \mathrm{rpm}$ for $24 \mathrm{~h}$. The crude mixture was then directly adsorbed onto diatomaceous earth (Celite ${ }^{\circledast}$ ) and purified by flash column chromatography (2.5-10\% ethyl acetate in hexanes). The product was obtained as a bright yellow oil (39.9 mg, 54\%).

$\mathrm{R}_{f}=0.12$ (5\% ethyl acetate in hexanes)

${ }^{1} \mathrm{H} \mathrm{NMR}\left(500 \mathrm{MHz}, \mathrm{CDCl}_{3}\right): \delta(\mathrm{ppm}) 8.11(\mathrm{~d}, J=8.8 \mathrm{~Hz}, 2 \mathrm{H}), 7.34(\mathrm{~d}, J=8.6 \mathrm{~Hz}, 2 \mathrm{H}), 7.23(\mathrm{~d}, J=8.3$ $\mathrm{Hz}, 2 \mathrm{H}), 6.82-6.73(\mathrm{~m}, 3 \mathrm{H}), 5.16(\mathrm{dd}, J=9.9,3.5 \mathrm{~Hz}, 1 \mathrm{H}), 3.90(\mathrm{dq}, J=10.8,7.2 \mathrm{~Hz}, 1 \mathrm{H}), 3.85(\mathrm{dq}, J=$ $10.8,7.2 \mathrm{~Hz}, 1 \mathrm{H}), 2.59(\mathrm{~s}, 3 \mathrm{H}), 2.54(\mathrm{dd}, J=14.4,9.9 \mathrm{~Hz}, 1 \mathrm{H}), 2.12(\mathrm{dd}, J=14.4,3.5 \mathrm{~Hz}, 1 \mathrm{H}), 1.31(\mathrm{~s}$, $3 \mathrm{H}), 1.29(\mathrm{~s}, 3 \mathrm{H}), 1.06(\mathrm{t}, J=7.1 \mathrm{~Hz}, 3 \mathrm{H})$

${ }^{13} \mathrm{C} \mathrm{NMR}\left(126 \mathrm{MHz}, \mathrm{CDCl}_{3}\right): \delta(\mathrm{ppm}) 177.18,149.79,148.66,147.22,129.54,128.11,123.77,118.23$, $114.43,60.94,58.94,42.22,41.60,32.91,26.98,25.63,14.15$

HRMS (ESI-TOF) $m / z:\left[\mathrm{M}+\mathrm{H}^{+}\right]$calculated for $\mathrm{C}_{21} \mathrm{H}_{27} \mathrm{~N}_{2} \mathrm{O}_{4}, 371.1971$; found, 371.1973

(Z)-3,3-dimethyl-5-(perfluorophenyl)-N-phenyldihydrofuran-2(3H)-imine (2d)

$\mathrm{PhN}$<smiles>CC1(C)CC(c2c(F)c(F)c(F)c(F)c2F)OC1=N</smiles>

In a nitrogen-filled glove box, an oven-dried 4-mL reaction vial was charged with $\mathrm{Cu}(\mathrm{OTf})_{2}(3.6 \mathrm{mg}, 10$ $\mu \mathrm{mol}, 5.0 \mathrm{~mol} \%), \mathrm{K}_{3} \mathrm{PO}_{4}(46.6 \mathrm{mg}, 0.220 \mathrm{mmol}, 1.10$ equiv), and a stir bar. Next, $400 \mu \mathrm{L}$ of a DCE stock solution containing 2,2'-bipyridine $(0.025 \mathrm{M}, 10 \mu \mathrm{mol}, 5.0 \mathrm{~mol} \%)$ was added. This was followed by addition of pentafluorostyrene $(27.6 \mu \mathrm{L}, 0.200 \mathrm{mmol}, 1.00$ equiv), aniline $(18.3 \mu \mathrm{L}, 0.200 \mathrm{mmol}, 1.00$ equiv), and ethyl 2-bromo-2-methylpropanoate ( $58.7 \mu \mathrm{L}, 0.400 \mathrm{mmol}, 2.00$ equiv). The vial was sealed 
with a Teflon-lined cap, removed from the glove box, and heated at $80^{\circ} \mathrm{C}$ with stirring at $660 \mathrm{rpm}$ for 24 h. The crude mixture was then directly adsorbed onto diatomaceous earth (Celite ${ }^{\circledast}$ ) and purified by flash column chromatography (5-10\% ethyl acetate in hexanes). The product was obtained as an off white solid (29.5 mg, 42\%).

$\mathrm{R}_{f}=0.14$ (10\% ethyl acetate in hexanes)

mp: $99.4-106^{\circ} \mathrm{C}$

${ }^{1} \mathrm{H} \mathrm{NMR}\left(500 \mathrm{MHz}, \mathrm{CDCl}_{3}\right): \delta(\mathrm{ppm}) 7.29-7.19(\mathrm{~m}, 2 \mathrm{H}), 7.06-6.97(\mathrm{~m}, 3 \mathrm{H}), 5.67(\mathrm{dd}, J=10.5,6.7$ $\mathrm{Hz}, 1 \mathrm{H}), 2.40(\mathrm{dd}, J=12.6,6.7 \mathrm{~Hz}, 1 \mathrm{H}), 2.33(\mathrm{dd}, J=12.6,10.5 \mathrm{~Hz}, 1 \mathrm{H}), 1.50(\mathrm{~s}, 3 \mathrm{H}), 1.47(\mathrm{~s}, 3 \mathrm{H})$

${ }^{13} \mathrm{C} \mathrm{NMR}\left(126 \mathrm{MHz}, \mathrm{CDCl}_{3}\right): \delta(\mathrm{ppm}) 166.74,147.17,145.46(\mathrm{~m}), 141.54(\mathrm{~m}), 137.99(\mathrm{~m}), 128.88$, $123.85,122.28,113.56(\mathrm{~m}), 70.71,44.39,41.85,26.87,26.28$

${ }^{19} \mathrm{~F} \mathrm{NMR}\left(470 \mathrm{MHz}, \mathrm{CDCl}_{3}\right): \delta(\mathrm{ppm})-143.36(\mathrm{~m}, 2 \mathrm{~F}),-153.43(\mathrm{t}, J=19.5 \mathrm{~Hz}, 1 \mathrm{~F}),-161.64(\mathrm{~m}, 2 \mathrm{~F})$

HRMS (ESI-TOF) $m / z:\left[\mathrm{M}+\mathrm{H}^{+}\right]$calculated for $\mathrm{C}_{18} \mathrm{H}_{15} \mathrm{~F}_{5} \mathrm{NO}$, 356.1074; found, 356.1065

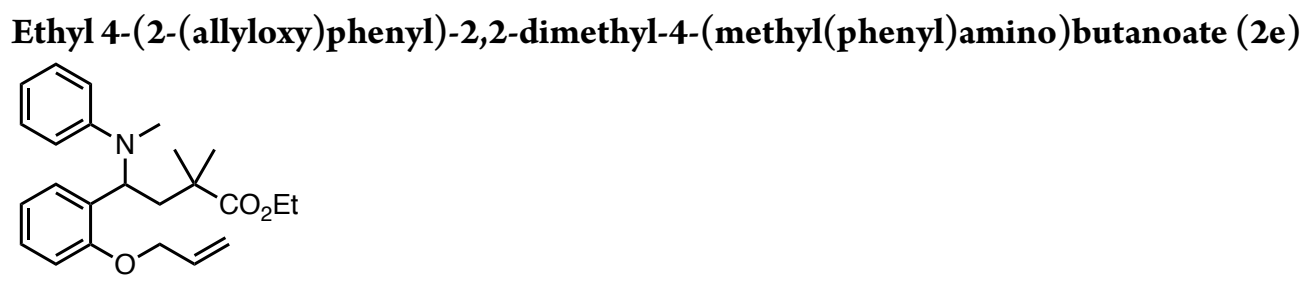

In a nitrogen-filled glove box, an oven-dried 4-mL reaction vial was charged with $\mathrm{Cu}(\mathrm{OTf})_{2}(1.8 \mathrm{mg}, 5.0$ $\mu \mathrm{mol}, 5.0 \mathrm{~mol} \%), \mathrm{K}_{3} \mathrm{PO}_{4}(23.3 \mathrm{mg}, 0.110 \mathrm{mmol}, 1.10$ equiv), and a stir bar. Next, $200 \mu \mathrm{L}$ of a DCE stock solution containing 2,2'-bipyridine $(0.025 \mathrm{M}, 5.0 \mu \mathrm{mol}, 5.0 \mathrm{~mol} \%)$ was added. This was followed by addition of 2-allyloxystyrene ( $16.0 \mathrm{mg}, 0.100 \mathrm{mmol}, 1.00$ equiv), $N$-methylaniline $(22.0 \mu \mathrm{L}, 0.200 \mathrm{mmol}$, 1.00 equiv), and ethyl 2-bromo-2-methylpropanoate $(29.4 \mu \mathrm{L}, 0.200 \mathrm{mmol}, 2.00$ equiv). The vial was sealed with a Teflon-lined cap, removed from the glove box, and heated at $80^{\circ} \mathrm{C}$ with stirring at $660 \mathrm{rpm}$ for $24 \mathrm{~h}$. The crude mixture was then directly adsorbed onto diatomaceous earth (Celite ${ }^{\circledR}$ ) and purified by flash column chromatography ( $2 \%$ ethyl acetate in hexanes). The product was obtained as a colorless oil (19.0 mg, 50\%).

$\mathrm{R}_{f}=0.20$ (2.5\% ethyl acetate in hexanes)

${ }^{1} \mathrm{H}$ NMR (500 MHz, $\left.\mathrm{CDCl}_{3}\right): \delta(\mathrm{ppm}) 7.26(\mathrm{dd}, J=7.6,1.5 \mathrm{~Hz}, 1 \mathrm{H}), 7.21-7.13(\mathrm{~m}, 3 \mathrm{H}), 6.93-6.78$ $(\mathrm{m}, 4 \mathrm{H}), 6.70-6.62(\mathrm{~m}, 1 \mathrm{H}), 5.94(\mathrm{ddt}, J=17.2,10.8,5.5 \mathrm{~Hz}, 1 \mathrm{H}), 5.54(\mathrm{dd}, J=8.2,5.3 \mathrm{~Hz}, 1 \mathrm{H}), 5.33$ (dddd, $J=17.3,1.6,1.6,1.6 \mathrm{~Hz}, 1 \mathrm{H}$ ), 5.24 (dddd, $J=10.5,1.4,1.4,1.4 \mathrm{~Hz}, 1 \mathrm{H}$ ), 4.48 (dddd, $J=12.6,5.7$, $1.5,1.5 \mathrm{~Hz}, 1 \mathrm{H}), 4.43$ (dddd, $J=12.6,5.4,1.6,1.5 \mathrm{~Hz}, 1 \mathrm{H}), 3.79(\mathrm{dq}, J=10.7,7.1 \mathrm{~Hz}, 1 \mathrm{H}), 3.72(\mathrm{dq}, J=$ $10.8,7.1 \mathrm{~Hz}, 1 \mathrm{H}), 2.75(\mathrm{~s}, 3 \mathrm{H}), 2.38(\mathrm{dd}, J=14.4,8.2 \mathrm{~Hz}, 1 \mathrm{H}), 2.11(\mathrm{dd}, J=14.4,5.3 \mathrm{~Hz}, 1 \mathrm{H}), 1.24(\mathrm{~s}$, $3 \mathrm{H}), 1.23(\mathrm{~s}, 3 \mathrm{H}), 1.02(\mathrm{t}, J=7.1 \mathrm{~Hz}, 3 \mathrm{H})$

${ }^{13} \mathrm{C} \mathrm{NMR}\left(126 \mathrm{MHz}, \mathrm{CDCl}_{3}\right): \delta$ (ppm) 177.53, 156.34, 150.27, 133.81, 130.03, 128.92, 128.18, 128.15, 120.32 , 117.90, 116.93, 114.36, 112.23, 69.44, 60.65, 52.57, 42.42, 41.58, 32.77, 26.82, 25.46, 14.09

HRMS (ESI-TOF) $m / z:\left[\mathrm{M}+\mathrm{H}^{+}\right]$calculated for $\mathrm{C}_{24} \mathrm{H}_{32} \mathrm{NO}_{3}, 382.2382$; found, 382.2385 
Ethyl 4-(2-(allyloxy)phenyl)-2,2-dimethyl-4-(phenylamino)butanoate (2f)<smiles>C=CCOc1ccccc1C(CC(C)(C)OCC)Nc1ccccc1</smiles>

In a nitrogen-filled glove box, an oven-dried 4-mL reaction vial was charged with $\mathrm{Cu}(\mathrm{OTf})_{2}(1.8 \mathrm{mg}, 5.0$ $\mu \mathrm{mol}, 5.0 \mathrm{~mol} \%), \mathrm{K}_{3} \mathrm{PO}_{4}(23.3 \mathrm{mg}, 0.110 \mathrm{mmol}, 1.10$ equiv), and a stir bar. Next, $200 \mu \mathrm{L}$ of a DCE stock solution containing 2,2'-bipyridine $(0.025 \mathrm{M}, 5.0 \mu \mathrm{mol}, 5.0 \mathrm{~mol} \%)$ was added. This was followed by addition of 2-allyloxystyrene ( $16.0 \mathrm{mg}, 0.100 \mathrm{mmol}, 1.00$ equiv), aniline $(9.10 \mu \mathrm{L}, 0.100 \mathrm{mmol}, 1.00$ equiv), and ethyl 2-bromo-2-methylpropanoate $(29.4 \mu \mathrm{L}, 0.200 \mathrm{mmol}, 2.00$ equiv). The vial was sealed with a Teflon-lined cap, removed from the glove box, and heated at $80^{\circ} \mathrm{C}$ with stirring at $660 \mathrm{rpm}$ for 24 $\mathrm{h}$. The crude mixture was then directly adsorbed onto diatomaceous earth (Celite ${ }^{\circledast}$ ) and purified by flash column chromatography ( $2.5 \%$ ethyl acetate in hexanes). The product was obtained as a colorless oil (18.5 mg, 50\%).

$\mathrm{R}_{f}=0.12(2.5 \%$ ethyl acetate in hexanes)

${ }^{1} \mathrm{H} \mathrm{NMR}\left(500 \mathrm{MHz}, \mathrm{CDCl}_{3}\right): \delta(\mathrm{ppm}) 7.26(\mathrm{dd}, J=7.4,1.5 \mathrm{~Hz}, 1 \mathrm{H}), 7.15(\mathrm{ddd}, J=7.8,1.6 \mathrm{~Hz}, 1 \mathrm{H})$, $7.08-7.01(\mathrm{~m}, 2 \mathrm{H}), 6.89-6.83(\mathrm{~m}, 2 \mathrm{H}), 6.58(\mathrm{t}, J=7.3 \mathrm{~Hz}, 1 \mathrm{H}), 6.45(\mathrm{~d}, J=7.8 \mathrm{~Hz}, 2 \mathrm{H}), 6.14(\mathrm{ddt}, J=$ $17.1,10.5,5.2 \mathrm{~Hz}, 1 \mathrm{H}), 5.48$ (dq, $J=17.3,1.5,1.5,1.5 \mathrm{~Hz}, 1 \mathrm{H}), 5.34$ (dddd, $J=10.5,1.3,1.3,1.3 \mathrm{~Hz}$, $1 \mathrm{H}), 4.92(\mathrm{dd}, J=10.3,3.1 \mathrm{~Hz}, 1 \mathrm{H}), 4.64(\mathrm{dddd}, J=13.0,5.3,1.6,1.3 \mathrm{~Hz}, 1 \mathrm{H}), 4.61$ (dddd, $J=13.0,5.3$, 1.6, $1.3 \mathrm{~Hz}, 1 \mathrm{H}), 4.40(\mathrm{~s}, 1 \mathrm{H}), 4.10(\mathrm{dq}, J=10.8,7.1 \mathrm{~Hz}, 1 \mathrm{H}), 4.04(\mathrm{dq}, J=10.8,7.1 \mathrm{~Hz}, 1 \mathrm{H}), 2.27(\mathrm{dd}, J$ $=14.4,10.4 \mathrm{~Hz}, 1 \mathrm{H}), 1.82(\mathrm{dd}, J=14.4,3.4 \mathrm{~Hz}, 1 \mathrm{H}), 1.31(\mathrm{~s}, 3 \mathrm{H}), 1.23(\mathrm{~s}, 3 \mathrm{H}), 1.19(\mathrm{t}, J=7.1 \mathrm{~Hz}, 3 \mathrm{H})$

${ }^{13} \mathrm{C} \mathrm{NMR}\left(126 \mathrm{MHz}, \mathrm{CDCl}_{3}\right): \delta(\mathrm{ppm}) 178.97,155.89,147.30,133.70,132.39,129.27,127.97,127.19$, $121.24,117.76,117.05,113.18,111.86,69.13,60.90,49.70,47.00,41.58,28.78,23.19,14.41$

HRMS (ESI-TOF) $m / z:\left[\mathrm{M}+\mathrm{H}^{+}\right]$calculated for $\mathrm{C}_{23} \mathrm{H}_{30} \mathrm{NO}_{3}, 368.2226$; found, 368.2225

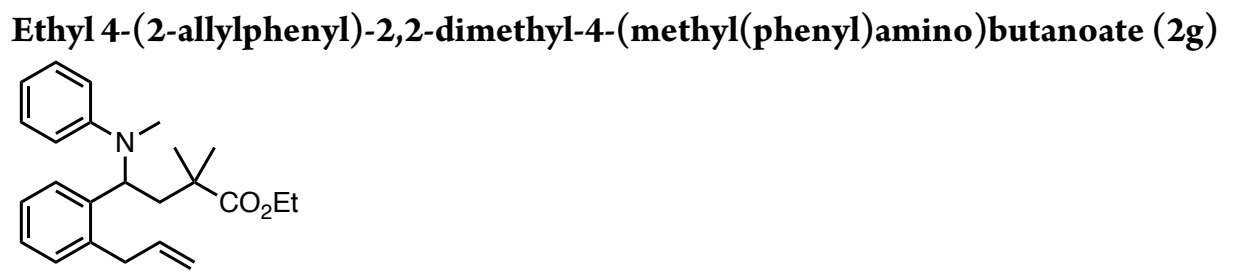

In a nitrogen-filled glove box, an oven-dried 4-mL reaction vial was charged with $\mathrm{Cu}(\mathrm{OTf})_{2}(1.8 \mathrm{mg}, 5.0$ $\mu \mathrm{mol}, 5.0 \mathrm{~mol} \%), \mathrm{K}_{3} \mathrm{PO}_{4}(23.3 \mathrm{mg}, 0.110 \mathrm{mmol}, 1.10$ equiv), and a stir bar. Next, $200 \mu \mathrm{L}$ of a DCE stock solution containing 2,2'-bipyridine $(0.025 \mathrm{M}, 5.0 \mu \mathrm{mol}, 5.0 \mathrm{~mol} \%)$ was added. This was followed by addition of 2-allylstyrene $(14.4 \mathrm{mg}, 0.100 \mathrm{mmol}, 1.00$ equiv), $N$-methylaniline $(11.0 \mu \mathrm{L}, 0.100 \mathrm{mmol}$, 1.00 equiv), and ethyl 2-bromo-2-methylpropanoate $(29.4 \mu \mathrm{L}, 0.200 \mathrm{mmol}, 2.00$ equiv). The vial was sealed with a Teflon-lined cap, removed from the glove box, and heated at $80^{\circ} \mathrm{C}$ with stirring at $660 \mathrm{rpm}$ for $24 \mathrm{~h}$. The crude mixture was then directly adsorbed onto diatomaceous earth (Celite ${ }^{\circledast}$ ) and purified by flash column chromatography ( $1 \%$ ethyl acetate in hexanes). The product was obtained as a colorless oil (19.4 mg, 53\%).

$\mathrm{R}_{f}=0.06$ ( $1 \%$ ethyl acetate in hexanes) 
${ }^{1} \mathrm{H} \operatorname{NMR}\left(500 \mathrm{MHz}, \mathrm{CDCl}_{3}\right): \delta(\mathrm{ppm}) 7.42-7.38(\mathrm{~m}, 1 \mathrm{H}), 7.25-7.19(\mathrm{~m}, 4 \mathrm{H}), 7.17-7.13(\mathrm{~m}, 1 \mathrm{H})$, $6.86(\mathrm{~d}, J=8.1 \mathrm{~Hz}, 2 \mathrm{H}), 6.75(\mathrm{t}, J=7.3 \mathrm{~Hz}, 1 \mathrm{H}), 5.88$ (dddd, $J=17.0,10.2,6.7,5.5 \mathrm{~Hz}, 1 \mathrm{H}), 5.26-5.19$ $(\mathrm{m}, 1 \mathrm{H}), 5.05(\mathrm{dddd}, J=10.3,1.7,1.7,1.7 \mathrm{~Hz}, 1 \mathrm{H}), 4.91(\mathrm{dddd}, J=17.1,1.8,1.8,1.8 \mathrm{~Hz}, 1 \mathrm{H}), 3.79(\mathrm{dq}, J$ $=10.8,7.1 \mathrm{~Hz}, 1 \mathrm{H}), 3.72(\mathrm{dq}, J=10.8,7.1 \mathrm{~Hz}, 1 \mathrm{H}), 3.33(\mathrm{dddd}, J=16.3,6.8,1.7,1.7 \mathrm{~Hz}, 1 \mathrm{H}), 3.13$ (dddd, $J=16.3,5.4,1.9,1.9 \mathrm{~Hz}, 1 \mathrm{H}), 2.55(\mathrm{~s}, 3 \mathrm{H}), 2.27(\mathrm{dd}, J=14.6,7.4 \mathrm{~Hz}, 1 \mathrm{H}), 2.19$ (dd, $J=14.6,6.4$ $\mathrm{Hz}, 1 \mathrm{H}), 1.23(\mathrm{~s}, 3 \mathrm{H}), 1.22(\mathrm{~s}, 3 \mathrm{H}), 1.07(\mathrm{t}, J=7.1 \mathrm{~Hz}, 3 \mathrm{H})$

${ }^{13} \mathrm{C} \mathrm{NMR}\left(126 \mathrm{MHz}, \mathrm{CDCl}_{3}\right): \delta$ (ppm) 177.43, 150.11, 139.44, 137.97, 137.55, 130.77, 129.35, 128.08, $127.55,125.74,117.73,116.07,114.77,60.65,55.32$, 41.42, 40.84, 37.20, 32.09, 26.67, 26.39, 14.14

HRMS (ESI-TOF) $m / z:\left[\mathrm{M}+\mathrm{H}^{+}\right]$calculated for $\mathrm{C}_{24} \mathrm{H}_{32} \mathrm{NO}_{2}$, 366.2433; found, 366.2417

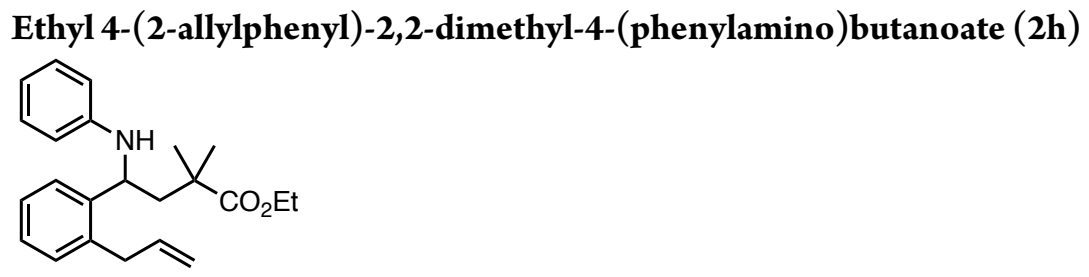

In a nitrogen-filled glove box, an oven-dried 4-mL reaction vial was charged with $\mathrm{Cu}(\mathrm{OTf})_{2}(1.8 \mathrm{mg}, 5.0$ $\mu \mathrm{mol}, 5.0 \mathrm{~mol} \%), \mathrm{K}_{3} \mathrm{PO}_{4}(23.3 \mathrm{mg}, 0.110 \mathrm{mmol}, 1.10$ equiv), and a stir bar. Next, $200 \mu \mathrm{L}$ of a DCE stock solution containing 2,2'-bipyridine $(0.025 \mathrm{M}, 5.0 \mu \mathrm{mol}, 5.0 \mathrm{~mol} \%)$ was added. This was followed by addition of 2-allylstyrene ( $14.4 \mathrm{mg}, 0.100 \mathrm{mmol}, 1.00$ equiv), aniline $(9.10 \mu \mathrm{L}, 0.100 \mathrm{mmol}, 1.00$ equiv), and ethyl 2-bromo-2-methylpropanoate $(29.4 \mu \mathrm{L}, 0.200 \mathrm{mmol}, 2.00$ equiv). The vial was sealed with a Teflon-lined cap, removed from the glove box, and heated at $80^{\circ} \mathrm{C}$ with stirring at $660 \mathrm{rpm}$ for $24 \mathrm{~h}$. The crude mixture was then directly adsorbed onto diatomaceous earth (Celite ${ }^{\oplus}$ ) and purified by flash column chromatography ( $1.5 \%$ ethyl acetate in hexanes). The product was obtained as a colorless oil (17.4 mg, 50\%).

$\mathrm{R}_{f}=0.05$ ( $1 \%$ ethyl acetate in hexanes)

${ }^{1} \mathrm{H} \operatorname{NMR}\left(500 \mathrm{MHz}, \mathrm{CDCl}_{3}\right): \delta(\mathrm{ppm}) 7.40-7.33(\mathrm{~m}, 1 \mathrm{H}), 7.20-7.12(\mathrm{~m}, 3 \mathrm{H}), 7.05-7.00(\mathrm{~m}, 2 \mathrm{H})$, $6.58(\mathrm{t}, J=7.3 \mathrm{~Hz}, 1 \mathrm{H}), 6.41(\mathrm{~d}, J=7.7 \mathrm{~Hz}, 2 \mathrm{H}), 6.04(\mathrm{dddd}, J=16.7,10.1,6.3,6.3 \mathrm{~Hz}, 1 \mathrm{H}), 5.17$ (dddd, $J=10.1,1.5,1.5,1.5 \mathrm{~Hz}, 1 \mathrm{H}), 5.12$ (dddd, $J=17.1,1.7,1.7,1.7 \mathrm{~Hz}, 1 \mathrm{H}), 4.76(\mathrm{dd}, J=11.0,3.1 \mathrm{~Hz}, 1 \mathrm{H})$, $4.40(\mathrm{~s}, 1 \mathrm{H}), 4.15(\mathrm{dq}, J=10.8,7.1 \mathrm{~Hz}, 1 \mathrm{H}), 4.10(\mathrm{dq}, J=10.8,7.1 \mathrm{~Hz}, 1 \mathrm{H}), 3.58$ (dddd, $J=16.1,6.7$, $1.5,1.5 \mathrm{~Hz}, 1 \mathrm{H}), 3.51$ (dddd, $J=15.7,5.9,1.7,1.7 \mathrm{~Hz}, 1 \mathrm{H}), 2.29(\mathrm{dd}, J=14.9,11.0 \mathrm{~Hz}, 1 \mathrm{H}), 1.69(\mathrm{dd}, J=$ 14.9, 3.2 Hz, 1H), $1.33(\mathrm{~s}, 3 \mathrm{H}), 1.24(\mathrm{~s}, 3 \mathrm{H}), 1.22(\mathrm{t}, J=7.2 \mathrm{~Hz}, 3 \mathrm{H})$

${ }^{13} \mathrm{C} \mathrm{NMR}\left(126 \mathrm{MHz}, \mathrm{CDCl}_{3}\right): \delta$ (ppm) 178.97, 146.95, 142.30, 137.20, 136.35, 130.33, 129.29, 127.27, 127.19, 125.70, 117.20, 116.71, 113.23, 61.14, 51.09, 48.03, 41.69, 36.73, 29.16, 22.98, 14.43

HRMS (ESI-TOF) $m / z:\left[\mathrm{M}+\mathrm{H}^{+}\right]$calculated for $\mathrm{C}_{23} \mathrm{H}_{30} \mathrm{NO}_{2}, 352.2277$; found, 352.2273

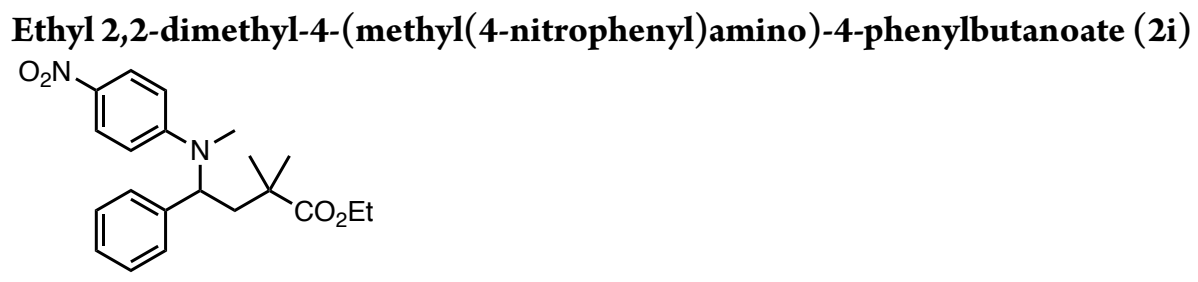


In a nitrogen-filled glove box, an oven-dried 4-mL reaction vial was charged with $\mathrm{Cu}(\mathrm{OTf})_{2}(3.6 \mathrm{mg}, 10$ $\mu \mathrm{mol}, 5.0 \mathrm{~mol} \%), \mathrm{K}_{3} \mathrm{PO}_{4}(46.6 \mathrm{mg}, 0.220 \mathrm{mmol}, 1.10$ equiv), and a stir bar. Next, $400 \mu \mathrm{L}$ of a DCE stock solution containing 2,2'-bipyridine $(0.025 \mathrm{M}, 10 \mu \mathrm{mol}, 5.0 \mathrm{~mol} \%)$ was added. This was followed by addition of styrene $(22.9 \mu \mathrm{L}, 0.200 \mathrm{mmol}, 1.00$ equiv), 4-nitro- $N$-methylaniline $(30.4 \mathrm{mg}, 0.200 \mathrm{mmol}$, 1.00 equiv), and ethyl 2-bromo-2-methylpropanoate ( $58.7 \mu \mathrm{L}, 0.400 \mathrm{mmol}, 2.00$ equiv). The vial was sealed with a Teflon-lined cap, removed from the glove box, and heated at $80^{\circ} \mathrm{C}$ with stirring at $660 \mathrm{rpm}$ for $24 \mathrm{~h}$. The crude mixture was then directly adsorbed onto diatomaceous earth (Celite ${ }^{\otimes}$ ) and purified by flash column chromatography ( $15 \%$ ethyl acetate in hexanes). The product was obtained as a bright yellow oil (44.2 mg, $60 \%)$.

$\mathrm{R}_{f}=0.40$ (20\% ethyl acetate in hexanes)

${ }^{1} \mathrm{H} \mathrm{NMR}\left(500 \mathrm{MHz}, \mathrm{CDCl}_{3}\right): \delta(\mathrm{ppm}) 8.13(\mathrm{~d}, J=9.5 \mathrm{~Hz}, 2 \mathrm{H}), 7.32(\mathrm{t}, J=7.3 \mathrm{~Hz}, 2 \mathrm{H}), 7.29-7.25(\mathrm{~m}$, $1 \mathrm{H}), 7.22(\mathrm{~d}, J=7.3 \mathrm{~Hz}, 2 \mathrm{H}), 6.77(\mathrm{~d}, J=9.5 \mathrm{~Hz}, 2 \mathrm{H}), 5.32(\mathrm{dd}, J=9.9,3.1 \mathrm{~Hz}, 1 \mathrm{H}), 3.88(\mathrm{dq}, J=10.8$, $7.1 \mathrm{~Hz}, 1 \mathrm{H}), 3.79(\mathrm{dq}, J=10.8,7.1 \mathrm{~Hz}, 1 \mathrm{H}), 2.74(\mathrm{~s}, 3 \mathrm{H}), 2.52(\mathrm{dd}, J=14.7,9.9 \mathrm{~Hz}, 1 \mathrm{H}), 2.22(\mathrm{dd}, J=$ $14.7,3.3 \mathrm{~Hz}, 1 \mathrm{H}), 1.28(\mathrm{~s}, 3 \mathrm{H}), 1.26(\mathrm{~s}, 3 \mathrm{H}), 1.04(\mathrm{t}, J=7.2 \mathrm{~Hz}, 3 \mathrm{H})$

${ }^{13} \mathrm{C} \mathrm{NMR}\left(126 \mathrm{MHz}, \mathrm{CDCl}_{3}\right): \delta(\mathrm{ppm}) 177.06,154.57,140.14,137.67,129.05,128.03,126.93,126.47$, $111.52,61.05,57.99,42.21,41.54,33.62,27.29,25.51,14.20$

HRMS (ESI-TOF) $m / z:\left[\mathrm{M}+\mathrm{H}^{+}\right]$calculated for $\mathrm{C}_{21} \mathrm{H}_{27} \mathrm{~N}_{2} \mathrm{O}_{4}, 371.1971$; found, 371.1985

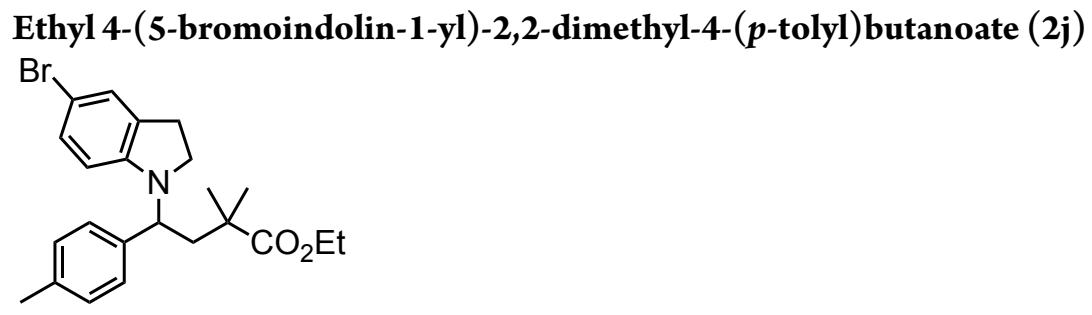

In a nitrogen-filled glove box, an oven-dried 4-mL reaction vial was charged with $\mathrm{Cu}(\mathrm{OTf})_{2}(3.6 \mathrm{mg}, 10$ $\mu \mathrm{mol}, 5.0 \mathrm{~mol} \%), \mathrm{K}_{3} \mathrm{PO}_{4}(46.6 \mathrm{mg}, 0.220 \mathrm{mmol}, 1.10$ equiv), and a stir bar. Next, $400 \mu \mathrm{L}$ of a DCE stock solution containing 2,2'-bipyridine $(0.025 \mathrm{M}, 10 \mu \mathrm{mol}, 5.0 \mathrm{~mol} \%)$ was added. This was followed by addition of 4-methylstyrene ( $26.3 \mu \mathrm{L}, 0.200 \mathrm{mmol}, 1.00$ equiv), 5-bromoindoline ( $39.6 \mathrm{mg}, 0.200 \mathrm{mmol}$, 1.00 equiv), and ethyl 2-bromo-2-methylpropanoate ( $58.7 \mu \mathrm{L}, 0.400 \mathrm{mmol}, 2.00$ equiv). The vial was sealed with a Teflon-lined cap, removed from the glove box, and heated at $80^{\circ} \mathrm{C}$ with stirring at $660 \mathrm{rpm}$ for $24 \mathrm{~h}$. The crude mixture was then directly adsorbed onto diatomaceous earth (Celite ${ }^{\otimes}$ ) and purified by flash column chromatography ( $2.5 \%$ ethyl acetate in hexanes). The product was obtained as a yellow oil (60.4 mg, 70\%).

$\mathrm{R}_{\mathrm{f}}=0.20$ (2.5\% ethyl acetate in hexanes)

${ }^{1} \mathrm{H} \mathrm{NMR}\left(500 \mathrm{MHz}, \mathrm{CDCl}_{3}\right): \delta(\mathrm{ppm}) 7.16-7.07(\mathrm{~m}, 5 \mathrm{H}), 7.02(\mathrm{~s}, 1 \mathrm{H}), 6.51(\mathrm{~d}, J=8.4 \mathrm{~Hz}, 1 \mathrm{H}), 4.79$ $(\mathrm{dd}, J=10.6,3.9 \mathrm{~Hz}, 1 \mathrm{H}), 3.83(\mathrm{dq}, J=10.9,7.1 \mathrm{~Hz}, 1 \mathrm{H}), 3.73(\mathrm{dq}, J=10.8,7.1 \mathrm{~Hz}, 1 \mathrm{H}), 3.41(\mathrm{td}, J=$ 9.0, $4.3 \mathrm{~Hz}, 1 \mathrm{H}), 2.96-2.89(\mathrm{~m}, 1 \mathrm{H}), 2.85-2.70(\mathrm{~m}, 2 \mathrm{H}), 2.62(\mathrm{dd}, J=14.1,10.6 \mathrm{~Hz}, 1 \mathrm{H}), 2.30(\mathrm{~s}$, $3 \mathrm{H}), 1.90(\mathrm{dd}, J=14.1,3.9 \mathrm{~Hz}, 1 \mathrm{H}), 1.28(\mathrm{~s}, 6 \mathrm{H}), 1.03(\mathrm{t}, J=7.1 \mathrm{~Hz}, 3 \mathrm{H})$

${ }^{13} \mathrm{C} \mathrm{NMR}\left(126 \mathrm{MHz}, \mathrm{CDCl}_{3}\right) \delta 177.44,150.40,137.37,136.36,132.33,129.92,129.22,127.98,127.66$, $108.40,108.23,77.30,60.55,55.23,46.58,42.10,41.14,28.09,27.92,24.37,21.29,14.06$ 
HRMS (ESI-TOF) $m / z:\left[\mathrm{M}+\mathrm{H}^{+}\right]$calculated for $\mathrm{C}_{23} \mathrm{H}_{29} \mathrm{NO}_{2} \mathrm{Br}, 430.1382$; found, 430.1370

\section{Ethyl 4-(diphenylamino)-2,2-dimethyl-4-phenylbutanoate (2k) \\ $\mathrm{Ph}_{\mathrm{N}^{\mathrm{N}}} \cdot \mathrm{Ph}$ \\ ${ }^{\mathrm{N}} \mathrm{C}_{\mathrm{CO}_{2} \mathrm{Et}}$}

In a nitrogen-filled glove box, an oven-dried 4-mL reaction vial was charged with $\mathrm{Cu}(\mathrm{OTf})_{2}(3.6 \mathrm{mg}, 10$ $\mu \mathrm{mol}, 5.0 \mathrm{~mol} \%), \mathrm{K}_{3} \mathrm{PO}_{4}(46.6 \mathrm{mg}, 0.220 \mathrm{mmol}, 1.10$ equiv), and a stir bar. Next, $400 \mu \mathrm{L}$ of a DCE stock solution containing 2,2'-bipyridine $(0.025 \mathrm{M}, 10 \mu \mathrm{mol}, 5.0 \mathrm{~mol} \%)$ was added. This was followed by addition of styrene $(22.9 \mu \mathrm{L}, 0.200 \mathrm{mmol}, 1.00$ equiv), diphenylamine $(33.8 \mu \mathrm{L}, 0.200 \mathrm{mmol}, 1.00$ equiv), and ethyl 2-bromo-2-methylpropanoate $(58.7 \mu \mathrm{L}, 0.400 \mathrm{mmol}, 2.00$ equiv). The vial was sealed with a Teflon-lined cap, removed from the glove box, and heated at $80^{\circ} \mathrm{C}$ with stirring at $660 \mathrm{rpm}$ for 24 $\mathrm{h}$. The crude mixture was then directly adsorbed onto diatomaceous earth (Celite ${ }^{\circledast}$ ) and purified by flash column chromatography ( $2.5 \%$ diethyl ether in hexanes). The product was obtained as a colorless solid (52.8 mg, 68\%).

$\mathrm{R}_{\mathrm{f}}=0.10$ (2.5\% diethyl ether in hexanes)

$\mathrm{mp}: 46.7-47.5^{\circ} \mathrm{C}$

${ }^{1} \mathrm{H} \mathrm{NMR}\left(500 \mathrm{MHz}, \mathrm{CDCl}_{3}\right): \delta(\mathrm{ppm}) 7.23-7.11(\mathrm{~m}, 9 \mathrm{H}), 6.94(\mathrm{t}, J=5 \mathrm{~Hz}, 2 \mathrm{H}), 6.84(\mathrm{~d}, J=10 \mathrm{~Hz}$, $4 \mathrm{H}), 5.39(\mathrm{dd}, J=7.7,5.2 \mathrm{~Hz}, 1 \mathrm{H}), 3.78(\mathrm{q}, J=7.1 \mathrm{~Hz}, 2 \mathrm{H}), 2.47(\mathrm{dd}, J=14.5,7.7 \mathrm{~Hz}, 1 \mathrm{H}), 2.39(\mathrm{dd}, J=$ $14.4,5.2 \mathrm{~Hz}, 1 \mathrm{H}), 1.26(\mathrm{~s}, 3 \mathrm{H}), 1.15(\mathrm{~s}, 3 \mathrm{H}), 1.11(\mathrm{t}, J=7.1 \mathrm{~Hz}, 3 \mathrm{H})$

${ }^{13} \mathrm{C}$ NMR (126 MHz, $\left.\mathrm{CDCl}_{3}\right): \delta$ (ppm) 177.55, 146.82, 140.96, 129.26, 128.99, 128.05, 127.36, 123.38, $121.95,60.73,59.91,42.77,42.07,27.38,25.43,14.25$

HRMS (ESI-TOF) $m / z:\left[\mathrm{M}+\mathrm{H}^{+}\right]$calculated for $\mathrm{C}_{26} \mathrm{H}_{30} \mathrm{NO}_{2}, 388.2277$; found, 388.2274

Ethyl 4-(9H-carbazol-9-yl)-4-(4-methoxyphenyl)-2,2-dimethylbutanoate (2l)<smiles>CCOC(=O)CC(c1ccc(OC)cc1)n1c2ccccc2c2ccccc21</smiles>

In a nitrogen-filled glove box, an oven-dried 4-mL reaction vial was charged with $\mathrm{Cu}(\mathrm{OTf})_{2}(3.6 \mathrm{mg}, 10$ $\mu \mathrm{mol}, 5.0 \mathrm{~mol} \%), \mathrm{K}_{3} \mathrm{PO}_{4}(46.6 \mathrm{mg}, 0.220 \mathrm{mmol}, 1.10$ equiv), and a stir bar. Next, $400 \mu \mathrm{L}$ of a DCE stock solution containing 2,2'-bipyridine $(0.025 \mathrm{M}, 10 \mu \mathrm{mol}, 5.0 \mathrm{~mol} \%)$ was added. This was followed by addition of 4-methoxystyrene $(26.6 \mu \mathrm{L}, 0.200 \mathrm{mmol}, 1.00$ equiv), carbazole $(33.4 \mu \mathrm{L}, 0.200 \mathrm{mmol}, 1.00$ equiv), and ethyl 2-bromo-2-methylpropanoate $(58.7 \mu \mathrm{L}, 0.400 \mathrm{mmol}, 2.00$ equiv). The vial was sealed with a Teflon-lined cap, removed from the glove box, and heated at $80^{\circ} \mathrm{C}$ with stirring at $660 \mathrm{rpm}$ for 24 $\mathrm{h}$. The crude mixture was then directly adsorbed onto diatomaceous earth (Celite ${ }^{\circledast}$ ) and purified by flash column chromatography ( $10 \%$ ethyl acetate in hexanes). The product was obtained as a colorless solid (63.3 mg, 76\%).

$\mathrm{R}_{\mathrm{f}}=0.30$ (10\% ethyl acetate in hexanes)

mp: $67.0-70.0^{\circ} \mathrm{C}$ 
${ }^{1} \mathrm{H} \mathrm{NMR}\left(500 \mathrm{MHz}, \mathrm{CDCl}_{3}\right): \delta(\mathrm{ppm}) 8.16-8.10(\mathrm{~m}, 2 \mathrm{H}), 7.50-7.36(\mathrm{~m}, 4 \mathrm{H}), 7.31-7.23(\mathrm{~m}, 2 \mathrm{H})$, $7.18(\mathrm{~d}, J=10 \mathrm{~Hz}, 2 \mathrm{H}), 6.79(\mathrm{~d}, J=10 \mathrm{~Hz}, 2 \mathrm{H}), 6.02(\mathrm{dd}, J=9.9,3.4 \mathrm{~Hz}, 1 \mathrm{H}), 3.76(\mathrm{~s}, 3 \mathrm{H}), 3.67(\mathrm{dq}, J=$ $10.7,7.1 \mathrm{~Hz}, 1 \mathrm{H}), 3.42(\mathrm{dq}, J=10.7,7.1 \mathrm{~Hz}, 1 \mathrm{H}), 3.15(\mathrm{dd}, J=14.5,9.9 \mathrm{~Hz}, 1 \mathrm{H}), 2.78(\mathrm{dd}, J=14.6,3.3$ $\mathrm{Hz}, 1 \mathrm{H}), 1.29(\mathrm{~s}, 3 \mathrm{H}), 1.02(\mathrm{~s}, 3 \mathrm{H}), 0.94(\mathrm{t}, J=7.2 \mathrm{~Hz}, 3 \mathrm{H})$

${ }^{13} \mathrm{C}$ NMR (126 MHz, $\left.\mathrm{CDCl}_{3}\right): \delta$ (ppm) 176.90, 158.88, 132.75, 127.90, 126.05, 125.70, 123.61, 120.56, $120.46,119.63$, 119.23, 114.04, 110.84, 77.55, 77.05, 60.64, 55.45, 53.90, 42.31, 41.94, 26.13, 25.86, 13.98

HRMS (ESI-TOF) $m / z$ : $\left[\mathrm{M}+\mathrm{H}^{+}\right]$calculated for $\mathrm{C}_{27} \mathrm{H}_{29} \mathrm{NO}_{3} \mathrm{Na}$, 438.2045; found, 438.2039

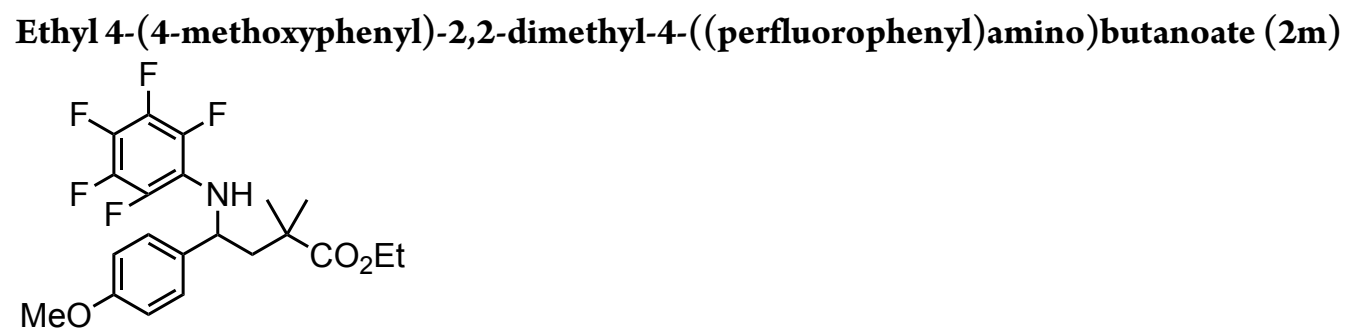

In a nitrogen-filled glove box, an oven-dried 4-mL reaction vial was charged with $\mathrm{Cu}(\mathrm{OTf})_{2}(3.6 \mathrm{mg}, 10$ $\mu \mathrm{mol}, 5.0 \mathrm{~mol} \%), \mathrm{K}_{3} \mathrm{PO}_{4}(46.6 \mathrm{mg}, 0.220 \mathrm{mmol}, 1.10$ equiv), and a stir bar. Next, $400 \mu \mathrm{L}$ of a DCE stock solution containing 2,2'-bipyridine $(0.025 \mathrm{M}, 10 \mu \mathrm{mol}, 5.0 \mathrm{~mol} \%)$ was added. This was followed by addition of 4-methoxystyrene $(26.9 \mu \mathrm{L}, 0.200 \mathrm{mmol}, 1.00$ equiv), 2,3,4,5,6-pentafluoroaniline (36.6 $\mathrm{mg}$, $0.200 \mathrm{mmol}, 1.00$ equiv), and ethyl 2-bromo-2-methylpropanoate ( $58.7 \mu \mathrm{L}, 0.400 \mathrm{mmol}, 2.00$ equiv). The vial was sealed with a Teflon-lined cap, removed from the glove box, and heated at $80{ }^{\circ} \mathrm{C}$ with stirring at $660 \mathrm{rpm}$ for $24 \mathrm{~h}$. The crude mixture was then directly adsorbed onto diatomaceous earth $\left(\right.$ Celite ${ }^{\odot}$ ) and purified by flash column chromatography (20\% ethyl acetate in hexanes). The product was obtained as a yellow oil ( $37.3 \mathrm{mg}, 44 \%)$.

$\mathrm{R}_{\mathrm{f}}=0.20$ (20\% ethyl acetate in hexanes)

${ }^{1} \mathrm{H}$ NMR $\left(400 \mathrm{MHz}, \mathrm{CDCl}_{3}\right): \delta(\mathrm{ppm}) 7.11(\mathrm{~d}, J=8.5 \mathrm{~Hz}, 2 \mathrm{H}), 6.80(\mathrm{~d}, J=8.5 \mathrm{~Hz}, 2 \mathrm{H}), 4.76-4.65(\mathrm{~m}$, $1 \mathrm{H}), 4.08(\mathrm{q}, J=7.1 \mathrm{~Hz}, 2 \mathrm{H}), 3.76(\mathrm{~s}, 3 \mathrm{H}), 2.39(\mathrm{dd}, J=14.6,10.6 \mathrm{~Hz}, 1 \mathrm{H}) 1.73(\mathrm{dd}, J=14.6,4.2 \mathrm{~Hz}$, $1 \mathrm{H}), 1.35(\mathrm{~s}, 3 \mathrm{H}), 1.29-1.19(\mathrm{~m}, 6 \mathrm{H})$

${ }^{13} \mathrm{C}$ NMR $\left(400 \mathrm{MHz}, \mathrm{CDCl}_{3}\right): \delta(\mathrm{ppm}) 178.43,159.19,138.50(\mathrm{~m}), 138.19(\mathrm{~m}), 135.51,135.13\left(\mathrm{tt}, J_{\mathrm{CF}}\right.$ $=14.3,3.9 \mathrm{~Hz}), 133.18(\mathrm{tt}, J=14.3,4.4 \mathrm{~Hz}), 127.25,122.87\left(\mathrm{t}, J_{\mathrm{CF}}=12.6 \mathrm{~Hz}\right), 114.35,61.02,56.89\left(\mathrm{t}, J_{\mathrm{CF}}\right.$ $=4.0 \mathrm{~Hz}), 55.48,48.52,41.40,28.58,23.32,14.32$

${ }^{19} \mathrm{~F} \operatorname{NMR}\left(470 \mathrm{MHz}, \mathrm{CDCl}_{3}\right): \delta(\mathrm{ppm})-158.08(\mathrm{~d}, J=22.1 \mathrm{~Hz}),-164.73(\mathrm{td}, J=22.4,4.7 \mathrm{~Hz}),-170.79$ $(\mathrm{tt}, J=22.1,5.8 \mathrm{~Hz})$

HRMS (ESI-TOF) $m / z:\left[\mathrm{M}+\mathrm{H}^{+}\right]$calculated for $\mathrm{C}_{21} \mathrm{H}_{22} \mathrm{NO}_{3} \mathrm{~F}_{5} \mathrm{Na}$, 454.1418; found, 454.1426

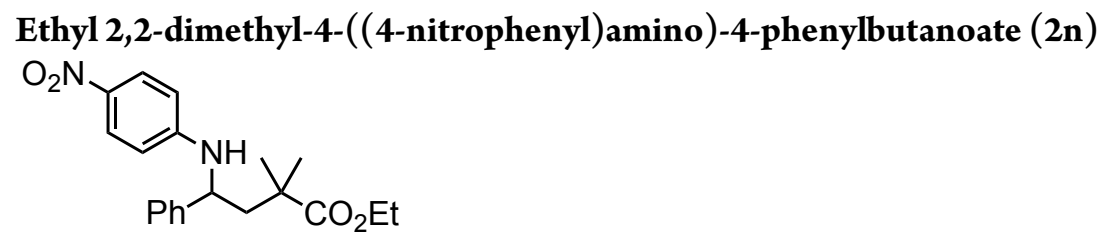


In a nitrogen-filled glove box, an oven-dried 4-mL reaction vial was charged with $\mathrm{Cu}(\mathrm{OTf})_{2}(3.6 \mathrm{mg}, 10$ $\mu \mathrm{mol}, 5.0 \mathrm{~mol} \%), \mathrm{K}_{3} \mathrm{PO}_{4}(46.6 \mathrm{mg}, 0.220 \mathrm{mmol}, 1.10$ equiv), and a stir bar. Next, $400 \mu \mathrm{L}$ of a DCE stock solution containing 2,2'-bipyridine $(0.025 \mathrm{M}, 10 \mu \mathrm{mol}, 5.0 \mathrm{~mol} \%)$ was added. This was followed by addition of styrene $(23.0 \mu \mathrm{L}, 0.200 \mathrm{mmol}, 1.00$ equiv), 4-nitroaniline $(27.6 \mathrm{mg}, 0.200 \mathrm{mmol}, 1.00$ equiv), and ethyl 2-bromo-2-methylpropanoate $(58.7 \mu \mathrm{L}, 0.400 \mathrm{mmol}, 2.00$ equiv). The vial was sealed with a Teflon-lined cap, removed from the glove box, and heated at $80^{\circ} \mathrm{C}$ with stirring at $660 \mathrm{rpm}$ for $24 \mathrm{~h}$. The crude mixture was then directly adsorbed onto diatomaceous earth (Celite $\left.{ }^{\oplus}\right)$ and purified by flash column chromatography (20\% ethyl acetate in hexanes). The product was obtained as a yellow oil (50.6 $\mathrm{mg}, 71 \%)$.

$\mathrm{R}_{\mathrm{f}}=0.30$ (20\% ethyl acetate in hexanes)

${ }^{1} \mathrm{H} \mathrm{NMR}\left(500 \mathrm{MHz}, \mathrm{CDCl}_{3}\right): \delta(\mathrm{ppm}) 7.97(\mathrm{~d}, J=8.9 \mathrm{~Hz}, 2 \mathrm{H}), 7.38-7.21(\mathrm{~m}, 5 \mathrm{H}), 6.40(\mathrm{~d}, J=9.1 \mathrm{~Hz}$, $2 \mathrm{H}), 5.56(\mathrm{~s}, 1 \mathrm{H}), 4.52(\mathrm{dd}, J=10.7,3.2 \mathrm{~Hz}, 1 \mathrm{H}), 4.18-4.06(\mathrm{~m}, 2 \mathrm{H}), 2.42(\mathrm{dd}, J=14.9,10.8 \mathrm{~Hz}, 1 \mathrm{H})$, $1.75(\mathrm{dd}, J=14.9,3.3 \mathrm{~Hz}, 1 \mathrm{H}), 1.31(\mathrm{~s}, 3 \mathrm{H}) 1.26(\mathrm{~s}, 3 \mathrm{H}), 1.22(\mathrm{t}, J=7.1 \mathrm{~Hz}, 3 \mathrm{H})$

${ }^{13} \mathrm{C}$ NMR $\left(126 \mathrm{MHz}, \mathrm{CDCl}_{3}\right) \delta 179.17,152.42,143.04,138.39,129.24,127.83,126.56,126.16,111.83$, $61.47,55.48,48.60,41.73,29.03,23.34,14.44$

HRMS (ESI-TOF) $m / z:\left[\mathrm{M}+\mathrm{H}^{+}\right]$calculated for $\mathrm{C}_{20} \mathrm{H}_{25} \mathrm{~N}_{2} \mathrm{O}_{4}, 357.1814$; found, 357.1821

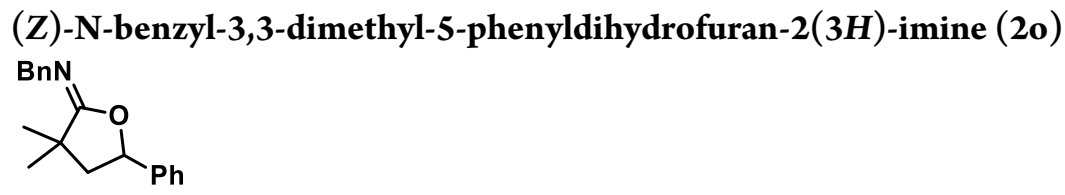

In a nitrogen-filled glove box, an oven-dried 4-mL reaction vial was charged with $\mathrm{Cu}(\mathrm{OTf})_{2}(3.6 \mathrm{mg}, 10$ $\mu \mathrm{mol}, 5.0 \mathrm{~mol} \%), \mathrm{K}_{3} \mathrm{PO}_{4}(46.6 \mathrm{mg}, 0.220 \mathrm{mmol}, 1.10$ equiv), and a stir bar. Next, $400 \mu \mathrm{L}$ of a DCE stock solution containing 2,2'-bipyridine $(0.025 \mathrm{M}, 10 \mu \mathrm{mol}, 5.0 \mathrm{~mol} \%)$ was added. This was followed by addition of styrene $(22.9 \mu \mathrm{L}, 0.200 \mathrm{mmol}, 1.00$ equiv), benzylamine ( $21.8 \mu \mathrm{L}, 0.200 \mathrm{mmol}, 1.00$ equiv), and ethyl 2-bromo-2-methylpropanoate $(58.7 \mu \mathrm{L}, 0.400 \mathrm{mmol}, 2.00$ equiv). The vial was sealed with a Teflon-lined cap, removed from the glove box, and heated at $80^{\circ} \mathrm{C}$ with stirring at $660 \mathrm{rpm}$ for $24 \mathrm{~h}$. The crude mixture was then directly adsorbed onto diatomaceous earth (Celite ${ }^{\otimes}$ ) and purified by flash column chromatography (10-20\% ethyl acetate in hexanes). The product was obtained as a colorless oil (38.0 mg, 68\%).

$\mathrm{R}_{f}=0.30$ (20\% ethyl acetate in hexanes)

${ }^{1} \mathrm{H}$ NMR $\left(500 \mathrm{MHz}, \mathrm{CDCl}_{3}\right): \delta(\mathrm{ppm}) 7.36(\mathrm{~m}, 4 \mathrm{H}), 7.30(\mathrm{~m}, 5 \mathrm{H}), 7.21(\mathrm{~m}, 1 \mathrm{H}), 5.37(\mathrm{dd}, J=10.3,5.9$ $\mathrm{Hz}, 1 \mathrm{H}), 4.60(\mathrm{~d}, J=15.1 \mathrm{~Hz}, 1 \mathrm{H}), 4.54(\mathrm{~d}, J=15.1 \mathrm{~Hz}, 1 \mathrm{H}), 2.37(\mathrm{dd}, J=12.6,5.9 \mathrm{~Hz}, 1 \mathrm{H}), 1.95(\mathrm{dd}, J$ $=12.6,10.2 \mathrm{~Hz}, 1 \mathrm{H}), 1.37(\mathrm{~s}, 3 \mathrm{H}), 1.34(\mathrm{~s}, 3 \mathrm{H})$

${ }^{13} \mathrm{C} \mathrm{NMR}\left(125 \mathrm{MHz}, \mathrm{CDCl}_{3}\right): \delta(\mathrm{ppm}) 168.97,141.59,141.03,128.88,128.44,128.29,127.79,126.45$, $125.73,79.32,51.09,48.11,41.34,26.65,26.54$

HRMS (ESI-TOF) $m / z$ : $\left[\mathrm{M}+\mathrm{H}^{+}\right]$calculated for $\mathrm{C}_{19} \mathrm{H}_{22} \mathrm{NO}, 280.1701$; found, 280.1704

(Z)-N-(4-methoxybenzyl)-3,3-dimethyl-5-(2-(thiophen-2-yl)phenyl)dihydrofuran-2(3H)-imine $(\mathbf{2 p})$ 


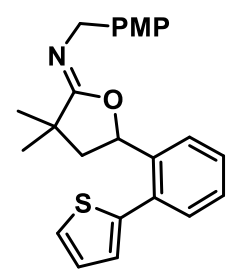

In a nitrogen-filled glove box, an oven-dried 4-mL reaction vial was charged with $\mathrm{Cu}(\mathrm{OTf})_{2}(3.6 \mathrm{mg}, 10$ $\mu \mathrm{mol}, 5.0 \mathrm{~mol} \%), \mathrm{K}_{3} \mathrm{PO}_{4}(46.6 \mathrm{mg}, 0.220 \mathrm{mmol}, 1.10$ equiv), and a stir bar. Next, $400 \mu \mathrm{L}$ of a DCE stock solution containing 2,2'-bipyridine ( $0.025 \mathrm{M}, 10 \mu \mathrm{mol}, 5.0 \mathrm{~mol} \%)$ was added. This was followed by addition of 2-(2-vinylphenyl)thiophene $(34.1 \mu \mathrm{L}, 0.200 \mathrm{mmol}, 1.00$ equiv), 4-methoxybenzylamine (26.1 $\mu \mathrm{L}, 0.200 \mathrm{mmol}, 1.00$ equiv), and ethyl 2-bromo-2-methylpropanoate ( $58.7 \mu \mathrm{L}, 0.400 \mathrm{mmol}, 2.00$ equiv). The vial was sealed with a Teflon-lined cap, removed from the glove box, and heated at $80{ }^{\circ} \mathrm{C}$ with stirring at $660 \mathrm{rpm}$ for $24 \mathrm{~h}$. The crude mixture was then directly adsorbed onto diatomaceous earth $\left(\right.$ Celite $\left.^{\circledast}\right)$ and purified by flash column chromatography (20\% ethyl acetate in hexanes). The product was obtained as a colorless solid (40.2 mg, 52\%).

$\mathrm{R}_{\mathrm{f}}=0.30$ (20\% ethyl acetate in hexanes)

mp: $98.5-99.3^{\circ} \mathrm{C}$

${ }^{1} \mathrm{H} \mathrm{NMR}\left(500 \mathrm{MHz}, \mathrm{CDCl}_{3}\right): \delta(\mathrm{ppm}) 7.46-7.35(\mathrm{~m}, 4 \mathrm{H}), 7.37-7.29(\mathrm{~m}, 1 \mathrm{H}), 7.28(\mathrm{~s}, 2 \mathrm{H}), 7.10(\mathrm{dd}$, $J=5.1,3.4 \mathrm{~Hz}, 1 \mathrm{H}), 7.03-6.98(\mathrm{~m}, 1 \mathrm{H}), 6.88-6.84(\mathrm{~m}, 2 \mathrm{H}), 5.63(\mathrm{dd}, J=10.2,5.8 \mathrm{~Hz}, 1 \mathrm{H}), 4.51(\mathrm{~d}, J$ $=14.6 \mathrm{~Hz}, 1 \mathrm{H}), 4.45(\mathrm{~d}, J=14.6 \mathrm{~Hz}, 1 \mathrm{H}), 3.80(\mathrm{~s}, 3 \mathrm{H}), 2.24(\mathrm{dd}, J=12.7,5.9 \mathrm{~Hz}, 1 \mathrm{H}), 1.93(\mathrm{dd}, J=$ $12.9,10.4 \mathrm{~Hz}, 1 \mathrm{H}) 1.31(\mathrm{~s}, 3 \mathrm{H}), 1.25(\mathrm{~s}, 3 \mathrm{H})$

${ }^{13} \mathrm{C}$ NMR $\left(126 \mathrm{MHz}, \mathrm{CDCl}_{3}\right) \delta 169.04,158.39,141.36,139.47,133.70,133.15,131.41,128.94,128.92$, $127.96,127.50,127.32,126.31,125.90,113.88,76.89,55.55,50.45,48.42,41.44,26.66,26.51$

HRMS (ESI-TOF) $m / z:\left[\mathrm{M}+\mathrm{H}^{+}\right]$calculated for $\mathrm{C}_{24} \mathrm{H}_{26} \mathrm{NO}_{2} \mathrm{~S}, 392.1684$; found, 392.1692

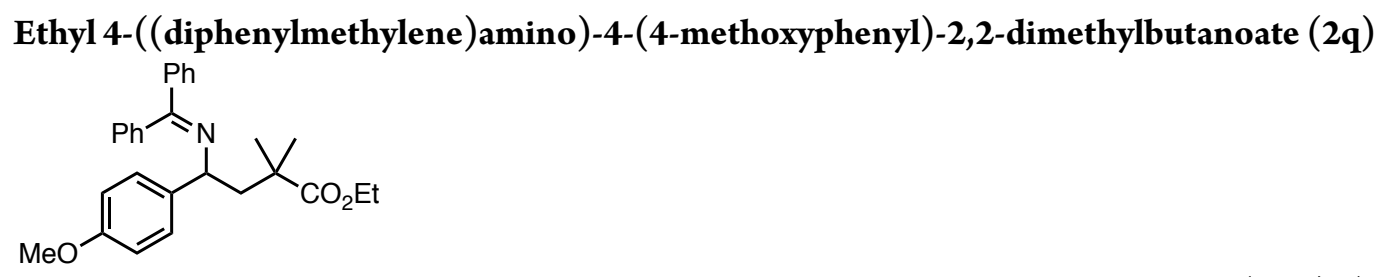

In a nitrogen-filled glove box, an oven-dried 4-mL reaction vial was charged with $\mathrm{Cu}(\mathrm{OTf})_{2}(3.6 \mathrm{mg}, 10$ $\mu \mathrm{mol}, 5.0 \mathrm{~mol} \%), \mathrm{K}_{3} \mathrm{PO}_{4}(46.6 \mathrm{mg}, 0.220 \mathrm{mmol}, 1.10$ equiv), and a stir bar. Next, $400 \mu \mathrm{L}$ of a DCE stock solution containing 2,2'-bipyridine $(0.025 \mathrm{M}, 10 \mu \mathrm{mol}, 5.0 \mathrm{~mol} \%)$ was added. This was followed by addition of 4-methoxystyrene ( $26.6 \mu \mathrm{L}, 0.200 \mathrm{mmol}, 1.00$ equiv), benzophenone imine (33.6 $\mu \mathrm{L}, 0.200$ mmol, 1.00 equiv), and ethyl 2-bromo-2-methylpropanoate ( $58.7 \mu \mathrm{L}, 0.400 \mathrm{mmol}, 2.00$ equiv). The vial was sealed with a Teflon-lined cap, removed from the glove box, and heated at $80^{\circ} \mathrm{C}$ with stirring at 660 $\mathrm{rpm}$ for $24 \mathrm{~h}$. The crude mixture was then directly adsorbed onto diatomaceous earth $\left(\mathrm{Celite}^{\oplus}\right)$ and purified by flash column chromatography (2.5-5.0\% ethyl acetate in hexanes). The product was obtained as a low melting solid $(71.0 \mathrm{mg}, 83 \%)$.

$\mathrm{R}_{f}=0.13$ ( $5 \%$ ethyl acetate in hexanes) 
${ }^{1} \mathrm{H}$ NMR $\left(500 \mathrm{MHz}, \mathrm{CDCl}_{3}\right): \delta(\mathrm{ppm}) 7.64(\mathrm{~d}, J=8.6 \mathrm{~Hz}, 2 \mathrm{H}), 7.45-7.38(\mathrm{~m}, 3 \mathrm{H}), 7.34(\mathrm{~d}, J=7.0 \mathrm{~Hz}$, $1 \mathrm{H}), 7.30(\mathrm{t}, J=7.3 \mathrm{~Hz}, 2 \mathrm{H}), 7.12(\mathrm{~d}, J=8.7 \mathrm{~Hz}, 2 \mathrm{H}), 7.04-6.98(\mathrm{~m}, 2 \mathrm{H}), 6.80(\mathrm{~d}, J=8.8 \mathrm{~Hz}, 2 \mathrm{H}), 4.40$ $(\mathrm{dd}, J=8.0,4.6 \mathrm{~Hz}, 1 \mathrm{H}), 3.86-3.72(\mathrm{~m}, 5 \mathrm{H}), 2.37(\mathrm{dd}, J=13.9,8.2 \mathrm{~Hz}, 1 \mathrm{H}), 2.06(\mathrm{dd}, J=14.0,4.5 \mathrm{~Hz}$, $1 \mathrm{H}), 1.11(\mathrm{~s}, 3 \mathrm{H}), 1.08(\mathrm{t}, J=7.1 \mathrm{~Hz}, 3 \mathrm{H}), 1.06(\mathrm{~s}, 3 \mathrm{H})$

${ }^{13} \mathrm{C} \mathrm{NMR}\left(126 \mathrm{MHz}, \mathrm{CDCl}_{3}\right): \delta(\mathrm{ppm}) 177.69,166.42,158.45,140.08,138.12,137.26,130.07,128.75$, $128.50,128.43,128.37,128.14,128.00,113.82,63.57,60.43,55.49,50.59,41.69,26.52,26.22,14.28$

HRMS (ESI-TOF) $m / z:\left[\mathrm{M}+\mathrm{H}^{+}\right]$calculated for $\mathrm{C}_{28} \mathrm{H}_{32} \mathrm{NO}_{3}, 430.2382$; found, 430.2389

1-(4-(4-Methoxyphenyl)-2,2-dimethyl-4-(methyl(phenyl)amino)butanoyl)pyrrolidin-2-one (2r)<smiles>COc1ccc(C(CC(C)(C)C(=O)N2CCCC2=O)N(C)c2ccccc2)cc1</smiles>

In a nitrogen-filled glove box, an oven-dried 4-mL reaction vial was charged with $\mathrm{Cu}(\mathrm{OTf})_{2}(1.8 \mathrm{mg}, 5.0$ $\mu \mathrm{mol}, 5.0 \mathrm{~mol} \%), \mathrm{K}_{3} \mathrm{PO}_{4}(23.3 \mathrm{mg}, 0.110 \mathrm{mmol}, 1.10$ equiv), and a stir bar. Next, $200 \mu \mathrm{L}$ of a DCE stock solution containing $N, N, N^{\prime}, N^{\prime}$-tetrakis(pyridin-2-ylmethyl)ethane-1,2-diamine (TPEN) (0.025 M, 5.0 $\mu \mathrm{mol}, 5.0 \mathrm{~mol} \%)$ was added. This was followed by addition of 4-methoxystyrene $(13.5 \mu \mathrm{L}, 0.100 \mathrm{mmol}$, 1.00 equiv), $N$-methylaniline $(11.0 \mu \mathrm{L}, \quad 0.100 \mathrm{mmol}, 1.00$ equiv), and 1-(2-bromo-2methylpropanoyl)pyrrolidin-2-one $(35.0 \mu \mathrm{L}, 0.200 \mathrm{mmol}, 2.00$ equiv). The vial was sealed with a Teflonlined cap, removed from the glove box, and heated at $80^{\circ} \mathrm{C}$ with stirring at $660 \mathrm{rpm}$ for $24 \mathrm{~h}$. The crude mixture was then directly adsorbed onto diatomaceous earth $\left(\right.$ Celite $\left.^{\oplus}\right)$ and purified by flash column chromatography (7.5-10\% ethyl acetate in hexanes). The product was obtained as a colorless oil (31.1 $\mathrm{mg}, 79 \%)$.

$\mathrm{R}_{f}=0.12$ ( $10 \%$ ethyl acetate in hexanes)

${ }^{1} \mathrm{H} \mathrm{NMR}\left(500 \mathrm{MHz}, \mathrm{CDCl}_{3}\right): \delta(\mathrm{ppm}) 7.23-7.16(\mathrm{~m}, 2 \mathrm{H}), 7.09(\mathrm{~d}, J=8.7 \mathrm{~Hz}, 2 \mathrm{H}), 6.76(\mathrm{~d}, J=8.7 \mathrm{~Hz}$, $2 \mathrm{H}), 6.77-6.74(\mathrm{~m}, 2 \mathrm{H}), 6.71(\mathrm{t}, J=7.2 \mathrm{~Hz}, 1 \mathrm{H}), 4.97(\mathrm{dd}, J=9.1,4.2 \mathrm{~Hz}, 1 \mathrm{H}), 3.75(\mathrm{~s}, 3 \mathrm{H}), 3.53(\mathrm{ddd}$, $J=11.7,8.2,6.0 \mathrm{~Hz}, 1 \mathrm{H}), 3.43(\mathrm{ddd}, J=11.7,8.2,6.4 \mathrm{~Hz}, 1 \mathrm{H}), 3.19(\mathrm{dd}, J=14.6,9.1 \mathrm{~Hz}, 1 \mathrm{H}), 2.56(\mathrm{~s}$, $3 \mathrm{H}), 2.45$ (ddd, $J=17.4,9.1,7.1 \mathrm{~Hz}, 1 \mathrm{H}), 2.39$ (ddd, $J=16.5,8.7,6.9 \mathrm{~Hz}, 1 \mathrm{H}), 2.27(\mathrm{dd}, J=14.6,4.2 \mathrm{~Hz}$, $1 \mathrm{H}), 1.82-1.74(\mathrm{~m}, 1 \mathrm{H}), 1.74-1.65(\mathrm{~m}, 1 \mathrm{H}), 1.47(\mathrm{~s}, 3 \mathrm{H}), 1.42(\mathrm{~s}, 3 \mathrm{H})$

${ }^{13} \mathrm{C} \mathrm{NMR}\left(126 \mathrm{MHz}, \mathrm{CDCl}_{3}\right): \delta(\mathrm{ppm}) 178.00,173.76,158.71,150.13,132.99,129.16,128.69,117.48$, $114.70,113.62,59.46,55.46,48.69,44.62,39.42,34.82,34.05,26.85,25.38,17.49$

HRMS (ESI-TOF) $m / z:\left[\mathrm{M}+\mathrm{H}^{+}\right]$calculated for $\mathrm{C}_{24} \mathrm{H}_{31} \mathrm{~N}_{2} \mathrm{O}_{3}, 395.2335$; found, 395.2350

\section{$N$-Methoxy- $N$,2,2-trimethyl-4-(methyl(phenyl)amino)-4-phenylbutanamide (2s)}<smiles>CON(C)C(=O)C(C)(CC(c1ccccc1)N(C)c1ccccc1)c1ccccc1</smiles>

In a nitrogen-filled glove box, an oven-dried $4-\mathrm{mL}$ reaction vial was charged with $\mathrm{Cu}(\mathrm{OTf})_{2}(3.6 \mathrm{mg}, 10$ $\mu \mathrm{mol}, 5.0 \mathrm{~mol} \%), \mathrm{K}_{3} \mathrm{PO}_{4}(46.6 \mathrm{mg}, 0.220 \mathrm{mmol}, 1.10$ equiv), and a stir bar. Next, $400 \mu \mathrm{L}$ of a DCE stock solution containing $N, N, N^{\prime}, N^{\prime \prime}, N^{\prime \prime}$-pentamethyldiethylenetriamine (PMDTA) ( $0.025 \mathrm{M}, 10 \mu \mathrm{mol}, 5.0$ mol \%) was added. This was followed by addition of styrene ( $22.9 \mu \mathrm{L}, 0.200 \mathrm{mmol}, 1.00$ equiv), $N$ - 
methylaniline (21.7 $\quad \mu \mathrm{L}, \quad 0.200 \quad \mathrm{mmol}, \quad 1.00 \quad$ equiv $), \quad$ and 2-bromo- $N$-methoxy- $N, 2-$ dimethylpropanamide $(60.6 \mu \mathrm{L}, 0.400 \mathrm{mmol}, 2.00$ equiv). The vial was sealed with a Teflon-lined cap, removed from the glove box, and heated at $80^{\circ} \mathrm{C}$ with stirring at $660 \mathrm{rpm}$ for $24 \mathrm{~h}$. The crude mixture was then directly adsorbed onto diatomaceous earth (Celite ${ }^{\otimes}$ ) and purified by flash column chromatography (10\% ethyl acetate in hexanes). The product was obtained as a clear oil ( $55.7 \mathrm{mg}, 82 \%)$.

$\mathrm{R}_{\mathrm{f}}=0.20$ (10\% ethyl acetate in hexanes)

${ }^{1} \mathrm{H} \mathrm{NMR}\left(500 \mathrm{MHz}, \mathrm{CDCl}_{3}\right): \delta(\mathrm{ppm}) 7.34-7.20(\mathrm{~m}, 7 \mathrm{H}), 6.86(\mathrm{dd}, J=8.9,1.1 \mathrm{~Hz}, 2 \mathrm{H}), 6.76(\mathrm{tt}, J=$ $7.1,0.9 \mathrm{~Hz}, 1 \mathrm{H}), 5.15(\mathrm{dd}, J=8.3,4.2 \mathrm{~Hz}, 1 \mathrm{H}), 3.64(\mathrm{~s}, 3 \mathrm{H}), 3.05(\mathrm{~s}, 3 \mathrm{H}), 2.69(\mathrm{~s}, 3 \mathrm{H}), 2.56(\mathrm{dd}, J=$ $14.4,8.3 \mathrm{~Hz}, 1 \mathrm{H}), 2.39$ (dd, $J=14.4,4.2 \mathrm{~Hz}, 1 \mathrm{H}), 1.39(\mathrm{~s}, 3 \mathrm{H}), 1.35(\mathrm{~s}, 3 \mathrm{H})$

${ }^{13} \mathrm{C}$ NMR $\left(126 \mathrm{MHz}, \mathrm{CDCl}_{3}\right) \delta 178.20,150.51,141.30,129.27,128.38,127.60,127.11,117.31,114.58$, $60.75,59.60,42.88,41.39,34.09,32.94,26.72,26.19$

HRMS (ESI-TOF) $m / z$ : $\left[\mathrm{M}+\mathrm{H}^{+}\right]$calculated for $\mathrm{C}_{21} \mathrm{H}_{29} \mathrm{~N}_{2} \mathrm{O}_{2}, 341.2229$; found, 341.2231

\section{Ethyl (E)-2,2-dimethyl-7-phenyl-7-(phenylamino)hept-4-enoate (2t)}<smiles>COc1ccc(C(CC(C)(C)C(=O)c2ccccc2)N(C)c2ccccc2)cc1</smiles>

In a nitrogen-filled glove box, an oven-dried 4-mL reaction vial was charged with $\mathrm{Cu}(\mathrm{OTf})_{2}(1.8 \mathrm{mg}, 5.0$ $\mu \mathrm{mol}, 5.0 \mathrm{~mol} \%), \mathrm{K}_{3} \mathrm{PO}_{4}(23.3 \mathrm{mg}, 0.110 \mathrm{mmol}, 1.10$ equiv), and a stir bar. Next, $200 \mu \mathrm{L}$ of a DCE stock solution containing $N, N, N^{\prime}, N^{\prime}$-tetrakis(pyridin-2-ylmethyl)ethane-1,2-diamine (TPEN) (0.025 M, 5.0 $\mu \mathrm{mol}, 5.0 \mathrm{~mol} \%)$. This was followed by addition of 4-methoxystyrene ( $13.5 \mu \mathrm{L}, 0.100 \mathrm{mmol}, 1.00$ equiv), $\mathrm{N}$-methylaniline ( $11.0 \mu \mathrm{L}, 0.100 \mathrm{mmol}, 1.00$ equiv), and ethyl 2-bromo-2-methyl-1-phenylpropan-1-one ( $45.4 \mathrm{mg}, 0.200 \mathrm{mmol}, 2.00$ equiv). The vial was sealed with a Teflon-lined cap, removed from the glove box, and heated at $80^{\circ} \mathrm{C}$ with stirring at $660 \mathrm{rpm}$ for $24 \mathrm{~h}$. The crude mixture was then directly adsorbed onto diatomaceous earth (Celite ${ }^{\odot}$ ) and purified by flash column chromatography (3\% ethyl acetate in hexanes). The product was obtained as a colorless oil, which solidified upon standing (17.4 mg, 45\%).

$\mathrm{R}_{f}=0.10$ (2.5\% ethyl acetate in hexanes)

mp: $75.9-78.2^{\circ} \mathrm{C}$

${ }^{1} \mathrm{H} \mathrm{NMR}\left(500 \mathrm{MHz}, \mathrm{CDCl}_{3}\right): \delta(\mathrm{ppm}) 7.45(\mathrm{~d}, J=7.8 \mathrm{~Hz}, 2 \mathrm{H}), 7.37(\mathrm{t}, J=7.4 \mathrm{~Hz}, 1 \mathrm{H}), 7.27(\mathrm{t}, J=7.8$ $\mathrm{Hz}, 2 \mathrm{H}), 7.19(\mathrm{t}, J=7.9 \mathrm{~Hz}, 2 \mathrm{H}), 7.10(\mathrm{~d}, J=8.7 \mathrm{~Hz}, 2 \mathrm{H}), 6.77(\mathrm{~d}, J=8.7 \mathrm{~Hz}, 2 \mathrm{H}), 6.74-6.69(\mathrm{~m}, 3 \mathrm{H})$, $5.12(\mathrm{dd}, J=10.2,3.5 \mathrm{~Hz}, 1 \mathrm{H}), 3.76(\mathrm{~s}, 3 \mathrm{H}), 2.85(\mathrm{dd}, J=14.5,10.2 \mathrm{~Hz}, 1 \mathrm{H}), 2.33(\mathrm{~s}, 3 \mathrm{H}), 2.26(\mathrm{dd}, J=$ $14.5,3.5 \mathrm{~Hz}, 1 \mathrm{H}), 1.42(\mathrm{~s}, 3 \mathrm{H}), 1.40(\mathrm{~s}, 3 \mathrm{H})$

${ }^{13} \mathrm{C}$ NMR (126 MHz, $\left.\mathrm{CDCl}_{3}\right): \delta(\mathrm{ppm}) 208.19,158.81,150.27,139.27,133.08,130.68,129.37,128.49$, $128.15,127.81,117.46,114.27,113.82,58.12,55.49,47.23,42.81,32.67,28.34,26.88$

HRMS (ESI-TOF) $m / z:\left[\mathrm{M}+\mathrm{H}^{+}\right]$calculated for $\mathrm{C}_{26} \mathrm{H}_{30} \mathrm{NO}_{2}$, 388.2277; found, 388.2263

Ethyl 2,2-difluoro-4-(4-methoxyphenyl)-4-(methyl(phenyl)amino)butanoate (2u) 
<smiles>CCOC(F)(F)CC(c1ccc(OC)cc1)N(C)c1ccccc1</smiles>

In a nitrogen-filled glove box, an oven-dried 4-mL reaction vial was charged with $\mathrm{Cu}(\mathrm{OTf})_{2}(3.6 \mathrm{mg}, 10$ $\mu \mathrm{mol}, 5.0 \mathrm{~mol} \%), \mathrm{K}_{3} \mathrm{PO}_{4}(46.6 \mathrm{mg}, 0.220 \mathrm{mmol}, 1.10$ equiv), and a stir bar. Next, $400 \mu \mathrm{L}$ of a DCE stock solution containing 2-(pyridin-2-yl)- $N$-(2-(pyridin-2-yl)ethyl)- $N$-(pyridin-2-ylmethyl)ethan-1-amine (DPEPA) $(0.025 \mathrm{M}, 10 \mu \mathrm{mol}, 5.0 \mathrm{~mol} \%)$ was added. This was followed by addition of 4-methoxystyrene ( $26.6 \mathrm{mg}, 0.200 \mathrm{mmol}, 1.00$ equiv), $N$-methylaniline ( $21.6 \mu \mathrm{L}, 0.200 \mathrm{mmol}, 1.00$ equiv), and ethyl 2bromo-2,2-difluoroacetate $(51.3 \mu \mathrm{L}, 0.400 \mathrm{mmol}, 2.00$ equiv). The vial was sealed with a Teflon-lined cap, removed from the glove box, and heated at $80{ }^{\circ} \mathrm{C}$ with stirring at $660 \mathrm{rpm}$ for $24 \mathrm{~h}$. The crude mixture was then directly adsorbed onto diatomaceous earth $\left(\mathrm{Celite}^{\oplus}\right)$ and purified by flash column chromatography (1.8-2.5\% ethyl acetate in hexanes). The product was obtained as a colorless oil contaminated with $5 \%$ of the Heck-type product, which was inseparable via flash column chromatography ( $34.5 \mathrm{mg}, 45 \%)$.

$\mathrm{R}_{f}=0.15$ (2.5\% ethyl acetate in hexanes)

Peaks for the Heck-type impurity are denoted by \{\}

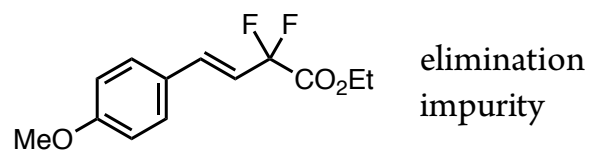

${ }^{1} \mathrm{H} \mathrm{NMR}\left(500 \mathrm{MHz}, \mathrm{CDCl}_{3}\right): \delta(\mathrm{ppm})\{7.39(\mathrm{~d}, J=8.7 \mathrm{~Hz}, 0.10 \mathrm{H})\}, 7.28-7.20(\mathrm{~m}, 2 \mathrm{H}), 7.02-6.95$ $(\mathrm{m}, 2 \mathrm{H}), 6.93-6.85(\mathrm{~m}, 2 \mathrm{H}), 6.83-6.77(\mathrm{~m}, 3 \mathrm{H}),\{6.16(\mathrm{dt}, J=16.2,11.5 \mathrm{~Hz}, 0.05 \mathrm{H})\}, 5.27(\mathrm{~m}, 1 \mathrm{H})$, $3.99(\mathrm{dq}, J=10.8,7.2 \mathrm{~Hz}, 2 \mathrm{H}),\{4.35(\mathrm{q}, J=7.1 \mathrm{~Hz}, 0.10 \mathrm{H})\}, 3.86(\mathrm{dq}, J=10.8,7.2 \mathrm{~Hz}, 2 \mathrm{H}), 3.77(\mathrm{~s}$, $3 \mathrm{H}), 3.27-3.09(\mathrm{~m}, 1 \mathrm{H}), 2.65-2.53(\mathrm{~m}, 1 \mathrm{H}), 2.35(\mathrm{~s}, 3 \mathrm{H}),\{1.36(\mathrm{t}, J=7.2 \mathrm{~Hz}, 0.15 \mathrm{H})\}, 0.99(\mathrm{t}, J=$ $7.1 \mathrm{~Hz}, 3 \mathrm{H})$

${ }^{13} \mathrm{C}$ NMR $\left(126 \mathrm{MHz}, \mathrm{CDCl}_{3}\right): \delta(\mathrm{ppm}) 163.86\left(\mathrm{t}, J^{\mathrm{C}-\mathrm{F}}=32.9 \mathrm{~Hz}\right), 159.33,150.45,130.00,129.33$, $128.28,118.97,116.51\left(\mathrm{dd}, J^{C-F}=256,244 \mathrm{~Hz}\right), 115.87,114.0,62.98,57.89\left(\mathrm{dd}, J^{C-F}=10.1,2.5 \mathrm{~Hz}\right)$, $55.51,36.46\left(\mathrm{t}, J^{C-F}=23.8 \mathrm{~Hz}\right), 31.98,13.77$

${ }^{19} \mathrm{~F} \mathrm{NMR}\left(470 \mathrm{MHz}, \mathrm{CDCl}_{3}\right): \delta(\mathrm{ppm})-100.24(\mathrm{~d}, J=261.3 \mathrm{~Hz}),\{-103.07(\mathrm{~d}, J=11.5 \mathrm{~Hz})\},-109.60$ $(\mathrm{dd}, J=261.6,27.8 \mathrm{~Hz})$

HRMS (ESI-TOF) $m / z:\left[\mathrm{M}+\mathrm{H}^{+}\right]$calculated for $\mathrm{C}_{20} \mathrm{H}_{24} \mathrm{~F}_{2} \mathrm{NO}_{3}, 364.1724$; found, 364.1723

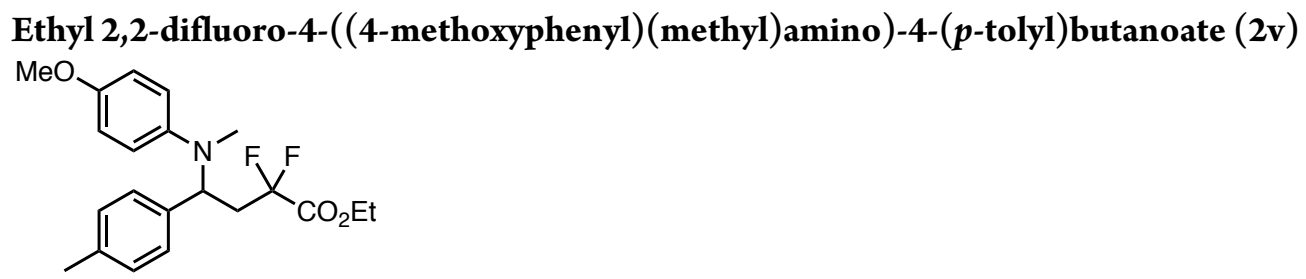

In a nitrogen-filled glove box, an oven-dried 4-mL reaction vial was charged with $\mathrm{Cu}(\mathrm{OTf})_{2}(3.6 \mathrm{mg}, 10$ $\mu \mathrm{mol}, 5.0 \mathrm{~mol} \%), \mathrm{K}_{3} \mathrm{PO}_{4}(46.6 \mathrm{mg}, 0.220 \mathrm{mmol}, 1.10$ equiv), and a stir bar. Next, $400 \mu \mathrm{L}$ of a DCE stock solution containing 2-(pyridin-2-yl)- $N$-(2-(pyridin-2-yl)ethyl)- $N$-(pyridin-2-ylmethyl)ethan-1-amine (DPEPA) $(0.025 \mathrm{M}, 10 \mu \mathrm{mol}, 5.0 \mathrm{~mol} \%)$ was added. This was followed by addition of 4-methylstyrene (23.6 mg, $0.200 \mathrm{mmol}, 1.00$ equiv), 4-methoxy- $\mathrm{N}$-methylaniline ( $27.4 \mathrm{mg}, 0.200 \mathrm{mmol}, 1.00$ equiv), and 
ethyl 2-bromo-2,2-difluoroacetate ( $51.3 \mu \mathrm{L}, 0.400 \mathrm{mmol}, 2.00$ equiv). The vial was sealed with a Teflonlined cap, removed from the glove box, and heated at $80{ }^{\circ} \mathrm{C}$ with stirring at $660 \mathrm{rpm}$ for $24 \mathrm{~h}$. The crude mixture was then directly adsorbed onto diatomaceous earth $\left(\right.$ Celite $\left.^{\circledast}\right)$ and purified by flash column chromatography ( $3 \%$ ethyl acetate in hexanes). The product was obtained as a colorless oil, which solidified upon standing $(31.7 \mathrm{mg}, 42 \%)$.

$\mathrm{R}_{f}=0.28$ (5\% ethyl acetate in hexanes)

$\mathrm{mp}: 43.2-44.6^{\circ} \mathrm{C}$

${ }^{1} \mathrm{H} \mathrm{NMR}\left(500 \mathrm{MHz}, \mathrm{CDCl}_{3}\right): \delta(\mathrm{ppm}) 7.05(\mathrm{~d}, J=7.8 \mathrm{~Hz}, 2 \mathrm{H}), 6.86(\mathrm{~d}, J=7.8 \mathrm{~Hz}, 2 \mathrm{H}), 6.84-6.80(\mathrm{~m}$, $4 \mathrm{H}), 5.02(\mathrm{dd}, J=11.9,2.5 \mathrm{~Hz}, 1 \mathrm{H}), 4.04(\mathrm{dq}, J=10.8,7.2 \mathrm{~Hz}, 1 \mathrm{H}), 3.96(\mathrm{dq}, J=10.8,7.2 \mathrm{~Hz}, 1 \mathrm{H}), 3.78$ $(\mathrm{s}, 3 \mathrm{H}), 3.30-3.15(\mathrm{~m}, 1 \mathrm{H}), 2.54-2.42(\mathrm{~m}, 1 \mathrm{H}), 2.30(\mathrm{~s}, 3 \mathrm{H}), 2.25(\mathrm{~s}, 3 \mathrm{H}), 1.07(\mathrm{t}, J=7.1 \mathrm{~Hz}, 3 \mathrm{H})$

${ }^{13} \mathrm{C} \mathrm{NMR}\left(126 \mathrm{MHz}, \mathrm{CDCl}_{3}\right): \delta(\mathrm{ppm}) 163.88\left(\mathrm{t}, J^{\mathrm{C}-\mathrm{F}}=32.6 \mathrm{~Hz}\right), 153.58,145.02,137.68,133.95$, $129.15,127.31,118.81,116.66\left(\mathrm{dd}, J^{C-F}=256,244 \mathrm{~Hz}\right), 114.55,62.84,60.54\left(\mathrm{dd}, J^{C-F}=9.7,2.3 \mathrm{~Hz}\right)$, $55.82,36.32\left(J^{C-F}=23.8 \mathrm{~Hz}\right), 32.27,21.33,13.92$

${ }^{19} \mathrm{~F} \mathrm{NMR}\left(470 \mathrm{MHz}, \mathrm{CDCl}_{3}\right): \delta(\mathrm{ppm})-99.75(\mathrm{ddd}, J=260.6,14.0,6.2 \mathrm{~Hz}),-109.99(\mathrm{ddd}, J=260.2$, $29.5,9.7 \mathrm{~Hz})$

HRMS (ESI-TOF) $m / z:\left[\mathrm{M}+\mathrm{H}^{+}\right]$calculated for $\mathrm{C}_{21} \mathrm{H}_{26} \mathrm{~F}_{2} \mathrm{NO}_{3}, 378.1881$; found, 378.1876

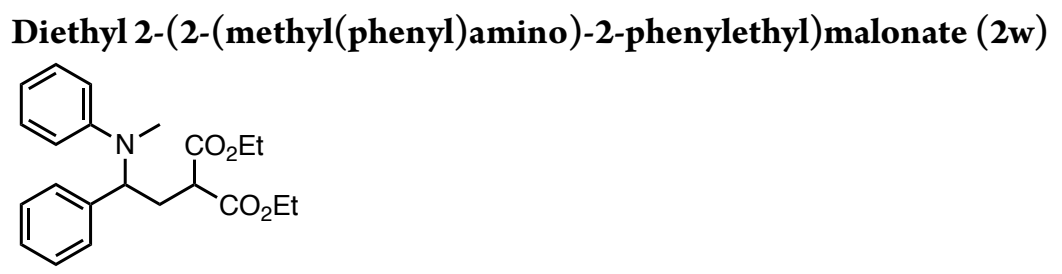

In a nitrogen-filled glove box, an oven-dried 4-mL reaction vial was charged with $\mathrm{Cu}(\mathrm{OTf})_{2}(3.6 \mathrm{mg}, 10$ $\mu \mathrm{mol}, 5.0 \mathrm{~mol} \%), \mathrm{K}_{3} \mathrm{PO}_{4}(46.6 \mathrm{mg}, 0.220 \mathrm{mmol}, 1.10$ equiv), and a stir bar. Next, $400 \mu \mathrm{L}$ of a DCE stock solution containing 2-(pyridin-2-yl)- $N$-(pyridin-2-ylmethyl)ethan-1-amine (PEPA) (0.025 M, $10 \mu \mathrm{mol}$, $5.0 \mathrm{~mol} \%)$ was added. This was followed by addition of styrene ( $114.6 \mu \mathrm{L}, 1.00 \mathrm{mmol}, 5.00$ equiv), $N$ methylaniline ( $21.6 \mu \mathrm{L}, 0.200 \mathrm{mmol}, 1.00$ equiv), and diethyl 2-bromomalonate $(34.1 \mu \mathrm{L}, 0.200 \mathrm{mmol}$, 1.00 equiv). The vial was sealed with a Teflon-lined cap, removed from the glove box, and heated at $80^{\circ} \mathrm{C}$ with stirring at $660 \mathrm{rpm}$ for $24 \mathrm{~h}$. The crude mixture was then directly adsorbed onto diatomaceous earth $\left(\right.$ Celite $\left.^{\circledast}\right)$ and purified by flash column chromatography (2.5-5.0\% ethyl acetate in hexanes). The product was obtained as a pale yellow oil ( $45.3 \mathrm{mg}, 61 \%)$.

$\mathrm{R}_{f}=0.15$ (5\% ethyl acetate in hexanes)

${ }^{1} \mathrm{H} \mathrm{NMR}\left(500 \mathrm{MHz}, \mathrm{CDCl}_{3}\right): \delta(\mathrm{ppm}) 7.34-7.16(\mathrm{~m}, 7 \mathrm{H}), 6.87-6.68(\mathrm{~m}, 3 \mathrm{H}), 5.13(\mathrm{~m}, 1 \mathrm{H}), 4.21-$ $4.05(\mathrm{~m}, 4 \mathrm{H}), 3.55-3.42(\mathrm{~m}, 1 \mathrm{H}), 2.74-2.63(\mathrm{~m}, 2 \mathrm{H}), 2.60(\mathrm{~s}, 3 \mathrm{H}), 1.19(\mathrm{td}, J=7.1,4.3 \mathrm{~Hz}, 6 \mathrm{H})$

${ }^{13} \mathrm{C} \mathrm{NMR}\left(126 \mathrm{MHz}, \mathrm{CDCl}_{3}\right): \delta(\mathrm{ppm}) 169.76,169.61,150.84,140.06,129.46,128.70,127.57,127.32$, $117.63,113.89,61.82,59.75,49.64,31.86,30.79,14.24$

HRMS (ESI-TOF) m/z: $\left[\mathrm{M}+\mathrm{H}^{+}\right]$calculated for $\mathrm{C}_{22} \mathrm{H}_{28} \mathrm{NO}_{4}, 370.2018$; found, 370.2027 
Ethyl (Z)-3-methyl-5-phenyl-2-(phenylimino)tetrahydrofuran-3-carboxylate (2x)

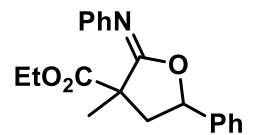

In a glovebox, an oven dried 4-mL vial was charged with $\mathrm{Cu}(\mathrm{MeCN})_{4} \mathrm{PF}_{6}(3.7 \mathrm{mg}, 10 \mu \mathrm{mol}, 5.0 \mathrm{~mol} \%)$, $\mathrm{Cs}_{2} \mathrm{CO}_{3}(71.7 \mathrm{mg}, 0.220 \mathrm{mmol}, 1.10$ equiv). A magnetic stir bar was added to the vial, followed by DCE $(0.400 \mathrm{~mL})$, PMDTA $(8.40 \mu \mathrm{L}, 0.0400 \mathrm{mmol}, 20.0 \mathrm{~mol} \%)$, styrene (23.0 $\mu \mathrm{L}, 0.200 \mathrm{mmol}, 1.00$ equiv), aniline (18.3 $\mu \mathrm{L}, 0.200 \mathrm{mmol}, 1.00$ equiv), and diethyl 2-bromo-2-methylmalonate. The vial was sealed with a Teflon septum screw-cap, brought outside of the glovebox, and heated at $80^{\circ} \mathrm{C}$ for 24 hours. The contents of the vial were diluted with ethyl acetate and combined with diatomaceous earth (Celite $\left.{ }^{\circledast}\right)$ in a $20 \mathrm{~mL}$ vial. Concentration by rotary evaporation yielded a dry powder, which was loaded directly onto a column of silica gel for flash column chromatography (7.5\% ethyl acetate in hexanes), which yields the isolable product diastereomers as colorless oils ( $51.7 \mathrm{mg}$ combined mass, $80 \%$ combined yield).

Diastereomer $1 \mathrm{R}_{\mathrm{f}}=0.30$ (7.5\% ethyl acetate in hexanes)

Diastereomer $2 \mathrm{R}_{\mathrm{f}}=0.20$ (7.5\% ethyl acetate in hexanes)

Crude d.r. $(\mathrm{GC})=2.5: 1$

Diastereomer 1: ${ }^{1} \mathrm{H}$ NMR (500 MHz, $\left.\mathrm{CDCl}_{3}\right): \delta(\mathrm{ppm}) 7.43-7.27(\mathrm{~m}, 7 \mathrm{H}), 7.16(\mathrm{dd}, J=8.4,1.3 \mathrm{~Hz}$, $2 \mathrm{H}), 7.06(\mathrm{tt}, J=7.2,1.2 \mathrm{~Hz}, 1 \mathrm{H}), 5.57(\mathrm{dd}, J=10.3,5.9 \mathrm{~Hz}, 1 \mathrm{H}), 4.37-4.26(\mathrm{~m}, 2 \mathrm{H}), 3.01(\mathrm{dd}, J=$ $13.2,6.0 \mathrm{~Hz}, 1 \mathrm{H}), 2.06(\mathrm{dd}, J=13.2,10.3 \mathrm{~Hz}, 1 \mathrm{H}), 1.68(\mathrm{~s}, 3 \mathrm{H}), 1.36(\mathrm{t}, J=7.2 \mathrm{~Hz}, 3 \mathrm{H})$

Diastereomer 1: ${ }^{13} \mathrm{C}$ NMR $\left(126 \mathrm{MHz}, \mathrm{CDCl}_{3}\right) \delta 172.58,162.26,146.92,139.87,128.96,128.80,128.61$, $125.75,124.10,122.98,81.51,62.19,52.98,45.08,22.49,14.48$

Diastereomer 1: HRMS (ESI-TOF) $m / z$ : $\left[\mathrm{M}+\mathrm{H}^{+}\right]$calculated for $\mathrm{C}_{20} \mathrm{H}_{22} \mathrm{NO}_{3}, 324.1600$; found, 324.1600

Diastereomer 2: ${ }^{1} \mathrm{H}$ NMR (500 MHz, $\left.\mathrm{CDCl}_{3}\right): \delta(\mathrm{ppm}) 7.44-7.31(\mathrm{~m}, 5 \mathrm{H}), 7.31-7.24(\mathrm{~m}, 2 \mathrm{H}), 7.14$ $(\mathrm{d}, J=7.8 \mathrm{~Hz}, 2 \mathrm{H}), 7.05(\mathrm{td}, J=7.4,1.4 \mathrm{~Hz}, 1 \mathrm{H}), 5.47(\mathrm{dd}, J=9.3,6.5 \mathrm{~Hz}, 1 \mathrm{H}), 4.31-4.21(\mathrm{~m}, 1 \mathrm{H})$, $4.21-4.12(\mathrm{~m}, 1 \mathrm{H}), 2.83(\mathrm{dd}, J=12.9,9.2 \mathrm{~Hz}, 1 \mathrm{H}), 2.48(\mathrm{dd}, J=12.8,6.4 \mathrm{~Hz}, 1 \mathrm{H}), 1.71(\mathrm{~s}, 3 \mathrm{H}), 1.29-$ $1.24(\mathrm{~m}, 3 \mathrm{H})$

Diastereomer $2:{ }^{13} \mathrm{C}$ NMR $\left(126 \mathrm{MHz}, \mathrm{CDCl}_{3}\right) \delta 172.07,162.89,146.50,139.39,128.65,128.52,128.41$, $125.71,123.86,122.77,81.31,61.76,52.40,43.99,21.58,14.05$

Diastereomer 2: HRMS (ESI-TOF) $m / z$ : $\left[\mathrm{M}+\mathrm{H}^{+}\right]$calculated for $\mathrm{C}_{20} \mathrm{H}_{22} \mathrm{NO}_{3}, 324.1600$; found, 324.1604

\section{Ethyl 4-(diphenylamino)-2-methyl-4-phenylbutanoate (2y)}<smiles>CCOC(=O)C(C)CC(c1ccccc1)[NH+](c1ccccc1)c1ccccc1</smiles>

In a nitrogen-filled glove box, an oven-dried 4-mL reaction vial was charged with $\mathrm{Cu}(\mathrm{OTf})_{2}(1.8 \mathrm{mg}, 5.0$ $\mu \mathrm{mol}, 5.0 \mathrm{~mol} \%), \mathrm{K}_{3} \mathrm{PO}_{4}(23.3 \mathrm{mg}, 0.110 \mathrm{mmol}, 1.10$ equiv), and a stir bar. Next, $200 \mu \mathrm{L}$ of a DCE stock solution containing 2,2'-bipyridine $(0.025 \mathrm{M}, 5.0 \mu \mathrm{mol}, 5.0 \mathrm{~mol} \%)$ was added. This was followed by addition of styrene $(11.5 \mu \mathrm{L}, 0.100 \mathrm{mmol}, 1.00$ equiv), diphenylamine $(16.9 \mathrm{mg}, 0.100 \mathrm{mmol}, 1.00$ 
equiv), and ethyl 2-bromopropanoate ( $26.0 \mu \mathrm{L}, 0.200 \mathrm{mmol}, 2.00$ equiv). The vial was sealed with a Teflon-lined cap, removed from the glove box, and heated at $80^{\circ} \mathrm{C}$ with stirring at $660 \mathrm{rpm}$ for $24 \mathrm{~h}$. The crude mixture was then directly adsorbed onto diatomaceous earth (Celite ${ }^{\oplus}$ ) and purified by flash column chromatography (1.5\% ethyl acetate in hexanes) to yield both product diastereomers (an inseparable mixture) as a colorless oil $(26.8 \mathrm{mg}, 72 \%)$.

Crude d.r. $(\mathrm{NMR})=2: 1$

$\mathrm{R}_{\mathrm{f}}=0.10$ (1.5\% ethyl acetate in hexanes)

${ }^{1} \mathrm{H}$ NMR (500 MHz, $\mathrm{CDCl}_{3}$, mixture of diastereomers): $\delta(\mathrm{ppm}) 7.33-7.12(\mathrm{~m}, 10 \mathrm{H}), 7.05-6.74(\mathrm{~m}$, $6 \mathrm{H}), 5.46(\mathrm{dd}, J=9.1,6.1 \mathrm{~Hz}, 0.5 \mathrm{H}), 5.36(\mathrm{dd}, J=8.9,6.2 \mathrm{~Hz}, 0.5 \mathrm{H}), 4.22-4.06(\mathrm{~m}, 2 \mathrm{H}), 2.61(\mathrm{dqd}, J$ $=14.0,7.1,4.5 \mathrm{~Hz}, 1 \mathrm{H}), 2.56-2.39(\mathrm{~m}, 1 \mathrm{H}), 2.11(\mathrm{ddd}, J=14.1,9.1,4.8 \mathrm{~Hz}, 0.5 \mathrm{H}), 1.98(\mathrm{ddd}, J=13.6$, $8.9,5.1 \mathrm{~Hz}, 0.5 \mathrm{H}), 1.30-1.20(\mathrm{~m}, 4 \mathrm{H}), 1.14(\mathrm{~d}, J=7.1 \mathrm{~Hz}, 2 \mathrm{H})$

${ }^{13} \mathrm{C}$ NMR (126 MHz, $\mathrm{CDCl}_{3}$, mixture of diastereomers) $\delta 176.81,176.60,147.00,146.82,141.67$, $140.85,129.30,128.70,128.47,128.42$, 128.05, 127.59, 127.32, 123.39, 123.09, 122.06, 121.95, 77.30, $60.73,60.69,60.23,59.79,36.96,36.90,36.02,35.93,18.23,18.14,14.54,14.50$

HRMS (ESI-TOF) $m / z:\left[\mathrm{M}+\mathrm{H}^{+}\right]$calculated for $\mathrm{C}_{25} \mathrm{H}_{28} \mathrm{NO}_{2}, 374.2120$; found, 374.2108

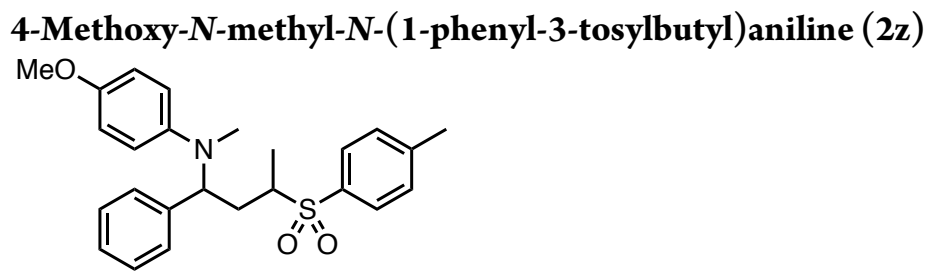

In a nitrogen-filled glove box, an oven-dried 4-mL reaction vial was charged with $\mathrm{Cu}(\mathrm{OTf})_{2}(3.6 \mathrm{mg}, 10$ $\mu \mathrm{mol}, 5.0 \mathrm{~mol} \%), \mathrm{K}_{3} \mathrm{PO}_{4}(46.6 \mathrm{mg}, 0.220 \mathrm{mmol}, 1.10$ equiv), and a stir bar. Next, $400 \mu \mathrm{L}$ of a DCE stock solution containing $N$-(2-(dimethylamino)ethyl)- $N, N^{\prime}, N^{\prime}$-trimethylethane-1,2-diamine (PMDTA) $(0.025 \mathrm{M}, 10 \mu \mathrm{mol}, 5.0 \mathrm{~mol} \%)$ was added. This was followed by addition of styrene $(22.9 \mu \mathrm{L}, 0.200$ mmol, 1.00 equiv), 4-methoxy- $N$-methylaniline $(27.4 \mathrm{mg}, 0.200 \mathrm{mmol}, 1.00$ equiv), and 1-((1bromoethyl)sulfonyl)-4-methylbenzene ( $105.4 \mathrm{mg}, 0.400 \mathrm{mmol}, 2.00$ equiv). The vial was sealed with a Teflon-lined cap, removed from the glove box, and heated at $80^{\circ} \mathrm{C}$ with stirring at $660 \mathrm{rpm}$ for $24 \mathrm{~h}$. The crude mixture was then directly adsorbed onto diatomaceous earth (Celite ${ }^{\circledast}$ ) and purified by flash column chromatography (10-20\% ethyl acetate in hexanes). The product was obtained as a pale yellow oil $(60.8 \mathrm{mg}, 67 \%)$. GC of the final isolated product indicated the d.r. to be $11: 1$. The final product contained $7 \%$ of tosyl ethane as an inseparable impurity.

$\mathrm{R}_{f}=0.20$ (10\% ethyl acetate in hexanes)

Crude d.r. (GC): 10:1

${ }^{1} \mathrm{H}$ NMR $\left(500 \mathrm{MHz}, \mathrm{CDCl}_{3}\right): \delta(\mathrm{ppm}) 7.76(\mathrm{~d}, J=8.2 \mathrm{~Hz}, 2 \mathrm{H}), 7.35(\mathrm{~d}, J=8.0 \mathrm{~Hz}, 2 \mathrm{H}), 7.30-7.20(\mathrm{~m}$, $3 \mathrm{H}), 7.11(\mathrm{~d}, J=6.6 \mathrm{~Hz}, 2 \mathrm{H}), 6.80(\mathrm{~d}, J=9.1 \mathrm{~Hz}, 2 \mathrm{H}), 6.72(\mathrm{~d}, J=9.1 \mathrm{~Hz}, 2 \mathrm{H}), 4.84(\mathrm{dd}, J=11.6,4.2$

$\mathrm{Hz}, 1 \mathrm{H}), 3.76(\mathrm{~s}, 3 \mathrm{H}), 3.24(\mathrm{dtt}, J=11.1,7.0,3.4 \mathrm{~Hz}, 1 \mathrm{H}), 2.77(\mathrm{ddd}, J=13.9,11.7,2.4 \mathrm{~Hz}, 1 \mathrm{H}), 2.45$

$(\mathrm{m}, 6 \mathrm{H}), 1.91(\mathrm{ddd}, J=14.4,10.5,4.3 \mathrm{~Hz}, 1 \mathrm{H}), 1.34(\mathrm{~d}, J=6.8 \mathrm{~Hz}, 3 \mathrm{H})$ 
${ }^{13} \mathrm{C} \mathrm{NMR}\left(126 \mathrm{MHz}, \mathrm{CDCl}_{3}\right): \delta(\mathrm{ppm}) 152.71,145.20,144.91,139.60,134.43,130.06,129.32,128.65$, $127.69,127.39,116.53,114.98,60.42,57.57,55.93,32.09,31.69,21.92,13.74$

HRMS (ESI-TOF) $m / z:\left[\mathrm{M}+\mathrm{H}^{+}\right]$calculated for $\mathrm{C}_{25} \mathrm{H}_{30} \mathrm{NO}_{3} \mathrm{~S}$, 424.1946; found, 424.1942

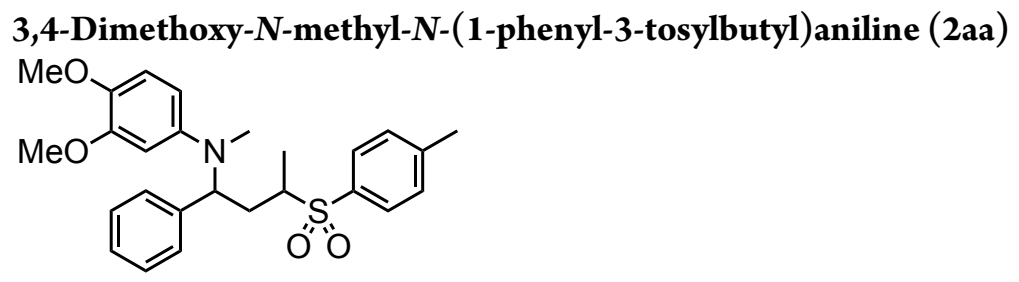

In a nitrogen-filled glove box, an oven-dried 4-mL reaction vial was charged with $\mathrm{Cu}(\mathrm{OTf})_{2}(3.6 \mathrm{mg}, 10$ $\mu \mathrm{mol}, 5.0 \mathrm{~mol} \%), \mathrm{K}_{3} \mathrm{PO}_{4}(46.6 \mathrm{mg}, 0.220 \mathrm{mmol}, 1.10$ equiv), and a stir bar. Next, $400 \mu \mathrm{L}$ of a DCE stock solution containing $\mathrm{N}$-(2-(dimethylamino)ethyl)- $N, N^{\prime}, N^{\prime}$-trimethylethane-1,2-diamine (PMDTA) $(0.025 \mathrm{M}, 10 \mu \mathrm{mol}, 5.0 \mathrm{~mol} \%)$ was added. This was followed by addition of styrene $(22.9 \mu \mathrm{L}, 0.200$ mmol, 1.00 equiv), 3,4-dimethoxy- $N$-methylaniline $(33.4 \mathrm{mg}, 0.200 \mathrm{mmol}, 1.00$ equiv), and $1-((1-$ bromoethyl)sulfonyl)-4-methylbenzene ( $105.4 \mathrm{mg}, 0.400 \mathrm{mmol}, 2.00$ equiv). The vial was sealed with a Teflon-lined cap, removed from the glove box, and heated at $80^{\circ} \mathrm{C}$ with stirring at $660 \mathrm{rpm}$ for $24 \mathrm{~h}$. The crude mixture was then directly adsorbed onto diatomaceous earth $\left(\mathrm{Celite}^{\oplus}\right)$ and purified by flash column chromatography (3-5\% diethyl ether in methylene chloride). The product was obtained as a pale yellow oil (44.9 mg, 50\%).

$\mathrm{R}_{f}=0.58$ ( $5 \%$ diethyl ether in methylene chloride)

Crude d.r. (GC): 10:1

${ }^{1} \mathrm{H}$ NMR $\left(500 \mathrm{MHz}, \mathrm{CDCl}_{3}\right): \delta(\mathrm{ppm}) 7.75(\mathrm{~d}, J=8.0 \mathrm{~Hz}, 2 \mathrm{H}), 7.34(\mathrm{~d}, J=8.0 \mathrm{~Hz}, 2 \mathrm{H}), 7.31-7.22(\mathrm{~m}$, $3 \mathrm{H}), 7.15(\mathrm{~d}, J=6.7 \mathrm{~Hz}, 2 \mathrm{H}), 6.76(\mathrm{~d}, J=8.7 \mathrm{~Hz}, 1 \mathrm{H}), 6.41(\mathrm{~d}, J=2.8 \mathrm{~Hz}, 1 \mathrm{H}), 6.29(\mathrm{dd}, J=8.8,2.8 \mathrm{~Hz}$, $1 \mathrm{H}), 4.89(\mathrm{dd}, J=11.3,4.5 \mathrm{~Hz}, 1 \mathrm{H}), 3.82(\mathrm{~s}, 3 \mathrm{H}), 3.82(\mathrm{~s}, 3 \mathrm{H}), 3.21$ (ddd, $J=9.9,6.8,2.9 \mathrm{~Hz}, 1 \mathrm{H}), 2.76$ (ddd, $J=14.1,11.4,2.8 \mathrm{~Hz}, 1 \mathrm{H}), 2.49$ (s, 3H), 2.44 (s, 3H), 1.94 (ddd, $J=14.4,10.1,4.5 \mathrm{~Hz}, 1 \mathrm{H}), 1.33$ $(\mathrm{d}, J=6.8 \mathrm{~Hz}, 3 \mathrm{H})$

${ }^{13} \mathrm{C}$ NMR $\left(126 \mathrm{MHz}, \mathrm{CDCl}_{3}\right): \delta(\mathrm{ppm}) 149.93,145.74,144.93,142.25,139.69,134.32,130.03,129.27$, $128.69,127.71,127.36,112.98,106.65,100.77,60.32$, 57.53, 56.70, 56.08, 32.43, 31.67, 21.89, 13.91

HRMS (ESI-TOF) $m / z:\left[\mathrm{M}+\mathrm{H}^{+}\right]$calculated for $\mathrm{C}_{26} \mathrm{H}_{32} \mathrm{NO}_{4} \mathrm{~S}$, 454.2052; found, 454.2054

\section{(Z)-5-hexyl-3,3-dimethyl- $N$-phenyldihydrofuran-2(3H)-imine \\ $\mathrm{PhN}$<smiles>C=C1OC(C)(C)CC1[AsH2+]</smiles>

In a nitrogen-filled glove box, an oven-dried 4-mL reaction vial was charged with $\mathrm{Cu}(\mathrm{OTf})_{2}(1.8 \mathrm{mg}, 5.0$ $\mu \mathrm{mol}, 5.0 \mathrm{~mol} \%), \mathrm{K}_{3} \mathrm{PO}_{4}(23.3 \mathrm{mg}, 0.110 \mathrm{mmol}, 1.10$ equiv), and a stir bar. Next, $200 \mu \mathrm{L}$ of a DCE stock solution containing 2,2'-bipyridine $(0.025 \mathrm{M}, 5.0 \mu \mathrm{mol}, 5.0 \mathrm{~mol} \%)$ was added. This was followed by addition of 1-octene ( $47.1 \mu \mathrm{L}, 0.300 \mathrm{mmol}, 3.00$ equiv), aniline $(9.10 \mu \mathrm{L}, 0.100 \mathrm{mmol}, 1.00$ equiv), and ethyl 2-bromo-2-methylpropanoate $(29.4 \mu \mathrm{L}, 0.200 \mathrm{mmol}, 2.00$ equiv). The vial was sealed with a Teflon-lined cap, removed from the glove box, and heated at $80^{\circ} \mathrm{C}$ with stirring at $660 \mathrm{rpm}$ for $24 \mathrm{~h}$. The crude mixture was then directly adsorbed onto diatomaceous earth $\left(\mathrm{Celite}^{\circledast}\right)$ and purified by flash 
column chromatography ( $5 \%$ ethyl acetate in hexanes). The product was obtained as a colorless oil (19.5 $\mathrm{mg}, 71 \%)$.

$\mathrm{R}_{f}=0.22$ (5\% ethyl acetate in hexanes)

${ }^{1} \mathrm{H} \mathrm{NMR}\left(500 \mathrm{MHz}, \mathrm{CDCl}_{3}\right): \delta(\mathrm{ppm}) 7.26(\mathrm{~m}, 2 \mathrm{H}), 7.07(\mathrm{~m}, 2 \mathrm{H}), 7.02(\mathrm{~m}, 1 \mathrm{H}), 4.37$ (dddd, $J=10.1$, 7.0, 6.2, $6.2 \mathrm{~Hz}, 1 \mathrm{H}), 2.08(\mathrm{dd}, J=12.4,5.6 \mathrm{~Hz}, 1 \mathrm{H}), 1.72(\mathrm{~m}, 1 \mathrm{H}), 1.67(\mathrm{dd}, J=12.4,10.3 \mathrm{~Hz}, 1 \mathrm{H}), 1.53$ $(\mathrm{m}, 1 \mathrm{H}), 1.37(\mathrm{~s}, 3 \mathrm{H}), 1.33(\mathrm{~s}, 3 \mathrm{H}), 1.27(\mathrm{~m}, 8 \mathrm{H}), 0.87(\mathrm{t}, J=6.9 \mathrm{~Hz}, 3 \mathrm{H})$

${ }^{13} \mathrm{C}$ NMR $\left(125 \mathrm{MHz}, \mathrm{CDCl}_{3}\right): \delta(\mathrm{ppm}) 168.80,147.79,128.67,123.45,123.20,79.62,44.69,41.73$, $35.79,31.95,29.40,26.82,26.77,25.60,22.83,14.35$ (two overlapping carbons)

HRMS (ESI-TOF) $m / z:\left[\mathrm{M}+\mathrm{H}^{+}\right]$calculated for $\mathrm{C}_{18} \mathrm{H}_{28} \mathrm{NO}, 274.2171$; found, 274.2178

(Z)-5-hexyl-N-(4-methoxybenzyl)-3,3-dimethyldihydrofuran-2(3H)-imine (2ac)

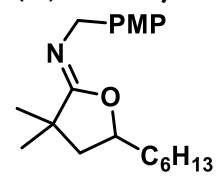

In a nitrogen-filled glove box, an oven-dried 4-mL reaction vial was charged with $\mathrm{Cu}(\mathrm{OTf})_{2}(3.6 \mathrm{mg}, 10$ $\mu \mathrm{mol}, 5.0 \mathrm{~mol} \%), \mathrm{K}_{3} \mathrm{PO}_{4}(46.6 \mathrm{mg}, 0.220 \mathrm{mmol}, 1.10$ equiv), and a stir bar. Next, $400 \mu \mathrm{L}$ of a DCE stock solution containing 2,2'-bipyridine (0.025 M, $10 \mu \mathrm{mol}, 5.0 \mathrm{~mol} \%)$ was added. This was followed by addition of 1-octene $(94.2 \mu \mathrm{L}, 0.600 \mathrm{mmol}, 3.00$ equiv), 4-methoxybenzylamine $(26.1 \mu \mathrm{L}, 0.200 \mathrm{mmol}$, 1.00 equiv), and ethyl 2-bromo-2-methylpropanoate ( $58.7 \mu \mathrm{L}, 0.400 \mathrm{mmol}, 2.00$ equiv). The vial was sealed with a Teflon-lined cap, removed from the glove box, and heated at $100^{\circ} \mathrm{C}$ with stirring at $660 \mathrm{rpm}$ for $24 \mathrm{~h}$. The crude mixture was then directly adsorbed onto diatomaceous earth (Celite ${ }^{\otimes}$ ) and purified by flash column chromatography ( $20 \%$ ethyl acetate in hexanes, visualized with the aid of $\mathrm{KMnO}_{4}$ stain). The product was obtained as a colorless oil $(19.6 \mathrm{mg}, 31 \%)$.

$\mathrm{R}_{\mathrm{f}}=0.20$ (20\% ethyl acetate in hexanes)

${ }^{1} \mathrm{H} \operatorname{NMR}\left(500 \mathrm{MHz}, \mathrm{CDCl}_{3}\right): \delta(\mathrm{ppm}) 7.23(\mathrm{~d}, J=8.9 \mathrm{~Hz}, 2 \mathrm{H}), 6.83(\mathrm{~d}, J=8.6 \mathrm{~Hz}, 2 \mathrm{H}), 4.42(\mathrm{~d}, J=$ $14.5 \mathrm{~Hz}, 1 \mathrm{H}), 4.37(\mathrm{~d}, J=14.5 \mathrm{~Hz}, 1 \mathrm{H}), 4.35-4.30(\mathrm{~m}, 1 \mathrm{H}), 3.78(\mathrm{~s}, 3 \mathrm{H}), 2.03$ (dd, $J=12.4,5.5 \mathrm{~Hz}$, $1 \mathrm{H}), 1.75-1.64(\mathrm{~m}, 1 \mathrm{H}), 1.60(\mathrm{dd}, J=12.4,10.2 \mathrm{~Hz}, 1 \mathrm{H}), 1.57-1.50(\mathrm{~m}, 1 \mathrm{H}), 1.48-1.39(\mathrm{~m}, 1 \mathrm{H})$, $1.38-1.21(\mathrm{~m}, 16 \mathrm{H}), 0.92-0.87(\mathrm{~m}, 3 \mathrm{H})$

${ }^{13} \mathrm{C}$ NMR $\left(126 \mathrm{MHz}, \mathrm{CDCl}_{3}\right) \delta 169.82,158.32,133.81,128.91,113.81,78.85,77.43,55.52,50.24,45.28$, $41.04,36.01,32.00,29.45,26.72,25.80,22.86,14.35$

HRMS (ESI-TOF) $m / z:\left[\mathrm{M}+\mathrm{H}^{+}\right]$calculated for $\mathrm{C}_{20} \mathrm{H}_{32} \mathrm{NO}_{2}, 318.2433$; found, 318.2438.

(Z)-5-(2-bromoethyl)-N-(4-bromophenyl)-3,3-dimethyldihydrofuran-2(3H)-imine (2ad)

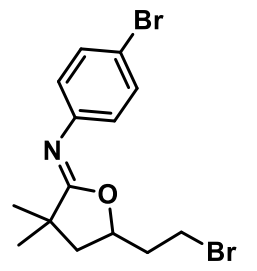


In a nitrogen-filled glove box, an oven-dried 4-mL reaction vial was charged with $\mathrm{Cu}(\mathrm{OTf})_{2}(3.6 \mathrm{mg}, 10$ $\mu \mathrm{mol}, 5.0 \mathrm{~mol} \%), \mathrm{K}_{3} \mathrm{PO}_{4}(46.6 \mathrm{mg}, 0.220 \mathrm{mmol}, 1.10$ equiv), and a stir bar. Next, $400 \mu \mathrm{L}$ of a DCE stock solution containing 2,2'-bipyridine $(0.025 \mathrm{M}, 10 \mu \mathrm{mol}, 5.0 \mathrm{~mol} \%)$ was added. This was followed by addition of 4-bromo-1-butene $(61.0 \mu \mathrm{L}, 0.600 \mathrm{mmol}, 3.00$ equiv), 4-bromoaniline $(34.4 \mathrm{mg}, 0.200 \mathrm{mmol}$, 1.00 equiv), and ethyl 2-bromo-2-methylpropanoate ( $58.7 \mu \mathrm{L}, 0.400 \mathrm{mmol}, 2.00$ equiv). The vial was sealed with a Teflon-lined cap, removed from the glove box, and heated at $80^{\circ} \mathrm{C}$ with stirring at $660 \mathrm{rpm}$ for $24 \mathrm{~h}$. The crude mixture was then directly adsorbed onto diatomaceous earth (Celite ${ }^{\otimes}$ ) and purified by flash column chromatography (10-20\% ethyl acetate in hexanes). The product was obtained as a pale yellow oil ( $33.7 \mathrm{mg}, 45 \%)$.

$\mathrm{R}_{f}=0.20$ (10\% ethyl acetate in hexanes)

${ }^{1} \mathrm{H} \mathrm{NMR}\left(500 \mathrm{MHz}, \mathrm{CDCl}_{3}\right): \delta(\mathrm{ppm}) 7.37(\mathrm{~d}, J=8.6 \mathrm{~Hz}, 2 \mathrm{H}), 6.97(\mathrm{~d}, J=8.6 \mathrm{~Hz}, 2 \mathrm{H}), 4.68-4.58(\mathrm{~m}$, $1 \mathrm{H}), 3.46-3.42(\mathrm{~m}, 2 \mathrm{H}), 2.22-2.15(\mathrm{~m}, 2 \mathrm{H}), 2.16-2.07(\mathrm{~m}, 1 \mathrm{H}), 1.73(\mathrm{dd}, J=12.5,10.2 \mathrm{~Hz}, 1 \mathrm{H})$, $1.40(\mathrm{~s}, 3 \mathrm{H}), 1.37(\mathrm{~s}, 3 \mathrm{H})$

${ }^{13} \mathrm{C} \mathrm{NMR}\left(126 \mathrm{MHz}, \mathrm{CDCl}_{3}\right): \delta(\mathrm{ppm}) 169.18,145.82,131.75,124.99,116.90,77.97,44.10,42.10$, $38.83,28.99,26.65,26.53$

HRMS (ESI-TOF) $m / z:\left[\mathrm{M}+\mathrm{H}^{+}\right]$calculated for $\mathrm{C}_{14} \mathrm{H}_{18} \mathrm{Br}_{2} \mathrm{NO}$, 373.9755; found, 373.9756

(Z)-3,3-dimethyl-5-(2-(oxiran-2-yl)ethyl)- $N$-(p-tolyl)dihydrofuran-2(3H)-imine (2ae)

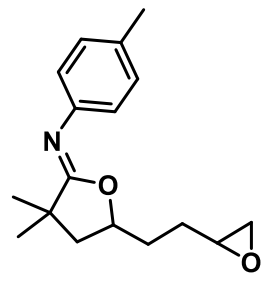

In a nitrogen-filled glove box, an oven-dried 4-mL reaction vial was charged with $\mathrm{Cu}(\mathrm{OTf})_{2}(3.6 \mathrm{mg}, 10$ $\mu \mathrm{mol}, 5.0 \mathrm{~mol} \%), \mathrm{K}_{3} \mathrm{PO}_{4}(46.6 \mathrm{mg}, 0.220 \mathrm{mmol}, 1.10$ equiv), and a stir bar. Next, $400 \mu \mathrm{L}$ of a DCE stock solution containing 2,2'-bipyridine $(0.025 \mathrm{M}, 10 \mu \mathrm{mol}, 5.0 \mathrm{~mol} \%)$ was added. This was followed by addition of 2-(but-3-en-1-yl)oxirane ( $113 \mu \mathrm{L}, 1.00 \mathrm{mmol}, 5.00$ equiv), 4-methylaniline (21.4 mg, 0.200 mmol, 1.00 equiv), and ethyl 2-bromo-2-methylpropanoate ( $146.8 \mu \mathrm{L}, 1.00 \mathrm{mmol}, 5.00$ equiv). The vial was sealed with a Teflon-lined cap, removed from the glove box, and heated at $80^{\circ} \mathrm{C}$ with stirring at 660 rpm for $24 \mathrm{~h}$. The crude mixture was then directly adsorbed onto diatomaceous earth (Celite ${ }^{\circledast}$ ) and purified by flash column chromatography (10-20\% ethyl acetate in hexanes). The product was obtained as a low melting solid ( $29.3 \mathrm{mg}, 54 \%, 1: 1$ d.r.).

$\mathrm{R}_{f}=0.25$ (20\% ethyl acetate in hexanes)

Crude D.R. (GC): 1:1

${ }^{1} \mathrm{H}$ NMR $\left(500 \mathrm{MHz}, \mathrm{CDCl}_{3}\right): \delta(\mathrm{ppm}) 7.06(\mathrm{~d}, J=8.0 \mathrm{~Hz}, 2 \mathrm{H}), 6.97(\mathrm{~d}, J=6.7 \mathrm{~Hz}, 2 \mathrm{H}), 4.45(\mathrm{dddd}, J=$ 6.6, 5.6, 5.6, $5.6 \mathrm{~Hz}, 0.5 \mathrm{H}), 4.39$ (dddd, $J=6.5,5.8,5.8,5.8 \mathrm{~Hz}, 0.5 \mathrm{H}), 2.95-2.92(\mathrm{~m}, 0.5 \mathrm{H}), 2.91-2.87$ $(\mathrm{m}, 0.5 \mathrm{H}), 2.74(\mathrm{dd}, J=5.1,4.0 \mathrm{~Hz}, 0.5 \mathrm{H}), 2.70(\mathrm{dd}, J=5.1,4.0 \mathrm{~Hz}, 0.5 \mathrm{H}), 2.47(\mathrm{dd}, J=4.9,2.7 \mathrm{~Hz}$, $0.5 \mathrm{H}), 2.45(\mathrm{dd}, J=4.9,2.7 \mathrm{~Hz}, 0.5 \mathrm{H}), 2.29(\mathrm{~s}, 3 \mathrm{H}), 2.12(\mathrm{dd}, J=5.6,1.5 \mathrm{~Hz}, 0.5 \mathrm{H}), 2.09(\mathrm{dd}, J=5.6,1.5$ $\mathrm{Hz}, 0.5 \mathrm{H}), 1.83-1.49(\mathrm{~m}, 5 \mathrm{H}), 1.37$ (apparent d, 3H), 1.33 (apparent d, 3H) 
${ }^{13} \mathrm{C} \mathrm{NMR}\left(126 \mathrm{MHz}, \mathrm{CDCl}_{3}\right): \delta(\mathrm{ppm}) 168.27,144.72,132.96,129.29,123.03,79.11,78.65,52.24$, 51.93 , 47.50, 47.27, 44.67, 44.50, 41.75, 41.72, 32.37, 31.95, 29.29, 28.59, 26.77, 26.72, 21.20

HRMS (ESI-TOF) $m / z:\left[\mathrm{M}+\mathrm{H}^{+}\right]$calculated for $\mathrm{C}_{17} \mathrm{H}_{24} \mathrm{NO}_{2}, 274.1807$; found, 274.1810

(Z)-N-([1,1'-biphenyl]-2-yl)-3,3-dimethyl-5-(2-(oxiran-2-yl)ethyl)dihydrofuran-2(3H)-imine (2af)

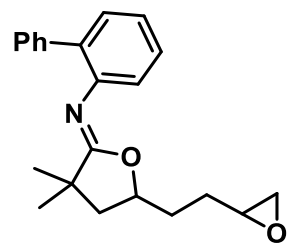

In a nitrogen-filled glove box, an oven-dried 4-mL reaction vial was charged with $\mathrm{Cu}(\mathrm{OTf})_{2}(3.6 \mathrm{mg}, 10$ $\mu \mathrm{mol}, 5.0 \mathrm{~mol} \%), \mathrm{K}_{3} \mathrm{PO}_{4}(46.6 \mathrm{mg}, 0.220 \mathrm{mmol}, 1.10$ equiv), and a stir bar. Next, $400 \mu \mathrm{L}$ of a DCE stock solution containing 2,2'-bipyridine $(0.025 \mathrm{M}, 10 \mu \mathrm{mol}, 5.0 \mathrm{~mol} \%)$ was added. This was followed by addition of 2-(but-3-en-1-yl) oxirane ( $113 \mu \mathrm{L}, 1.00 \mathrm{mmol}, 5.00$ equiv), 2-phenylaniline (33.8 mg, 0.200 mmol, 1.00 equiv), and ethyl 2-bromo-2-methylpropanoate $(146.8 \mu \mathrm{L}, 1.00 \mathrm{mmol}, 5.00$ equiv). The vial was sealed with a Teflon-lined cap, removed from the glove box, and heated at $80^{\circ} \mathrm{C}$ with stirring at 660 rpm for $24 \mathrm{~h}$. The crude mixture was then directly adsorbed onto diatomaceous earth $\left(\mathrm{Celite}^{\oplus}\right)$ and purified by flash column chromatography (10-20\% ethyl acetate in hexanes). The product was obtained as a low melting solid (30.8 mg, 46\%, 1:1 d.r.).

$\mathrm{R}_{f}=0.28$ (20\% ethyl acetate in hexanes)

Crude D.R. (GC): 1:1

${ }^{1} \mathrm{H} \mathrm{NMR}\left(500 \mathrm{MHz}, \mathrm{CDCl}_{3}\right): \delta(\mathrm{ppm}) 7.44-7.38(\mathrm{~m}, 2 \mathrm{H}), 7.32(\mathrm{t}, J=7.6 \mathrm{~Hz}, 2 \mathrm{H}), 7.29-7.22(\mathrm{~m}$, $3 \mathrm{H}), 7.10(\mathrm{t}, J=7.5 \mathrm{~Hz}, 1 \mathrm{H}), 6.95(\mathrm{~d}, J=7.8 \mathrm{~Hz}, 1 \mathrm{H}), 4.11(\mathrm{~m}, 0.5 \mathrm{H}), 4.04(\mathrm{~m}, 0.5 \mathrm{H}), 2.86-2.81(\mathrm{~m}$, $0.5 \mathrm{H}), 2.81-2.77(\mathrm{~m}, 0.5 \mathrm{H}), 2.70(\mathrm{dd}, J=5.2,4.2 \mathrm{~Hz}, 0.5 \mathrm{H}), 2.65(\mathrm{dd}, J=5.2,4.2 \mathrm{~Hz}, 0.5 \mathrm{H}), 2.40(\mathrm{dd}, J$ $=5.2,2.7 \mathrm{~Hz}, 0.5 \mathrm{H}), 2.39(\mathrm{dd}, J=5.2,2.7 \mathrm{~Hz}, 0.5 \mathrm{H}), 1.92(\mathrm{dd}, J=5.5,2.7 \mathrm{~Hz}, 0.5 \mathrm{H}), 1.89(\mathrm{dd}, J=5.5$, $2.7 \mathrm{~Hz}, 0.5 \mathrm{H}), 1.48-1.30$ (m, 5H), 1.24 (s, 3H), 1.13 (app. d, 3H)

${ }^{13} \mathrm{C} \mathrm{NMR}\left(126 \mathrm{MHz}, \mathrm{CDCl}_{3}\right): \delta$ (ppm) 167.51, 141.11, 134.12, 130.16, 129.57, 127.96, 127.80, 126.69, $126.66,123.66,122.46,78.90,78.38,52.20,51.82$, 47.43, 47.14, 44.69, 44.51, 41.29, 32.10, 31.63, 29.27, $28.48,26.37,26.26$

HRMS (ESI-TOF) $m / z:\left[\mathrm{M}+\mathrm{H}^{+}\right]$calculated for $\mathrm{C}_{22} \mathrm{H}_{26} \mathrm{NO}_{2}, 336.1964$; found, 336.1972

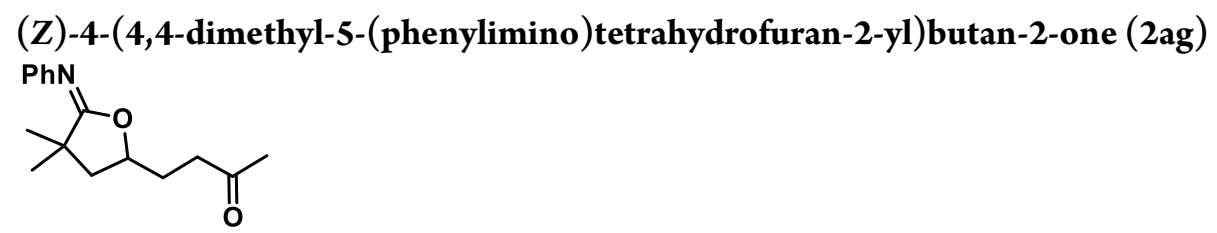

In a nitrogen-filled glove box, an oven-dried 4-mL reaction vial was charged with $\mathrm{Cu}(\mathrm{OTf})_{2}(3.6 \mathrm{mg}, 10$ $\mu \mathrm{mol}, 5.0 \mathrm{~mol} \%), \mathrm{K}_{3} \mathrm{PO}_{4}(46.6 \mathrm{mg}, 0.220 \mathrm{mmol}, 1.10$ equiv), and a stir bar. Next, $400 \mu \mathrm{L}$ of a DCE stock solution containing 2,2'-bipyridine $(0.025 \mathrm{M}, 10 \mu \mathrm{mol}, 5.0 \mathrm{~mol} \%)$ was added. This was followed by addition of 5-hexen-2-one ( $68.8 \mu \mathrm{L}, 0.600 \mathrm{mmol}, 3.00$ equiv), aniline ( $18.3 \mu \mathrm{L}, 0.200 \mathrm{mmol}, 1.00$ equiv), and ethyl 2-bromo-2-methylpropanoate $(58.7 \mu \mathrm{L}, 0.400 \mathrm{mmol}, 2.00$ equiv). The vial was sealed with a 
Teflon-lined cap, removed from the glove box, and heated at $80^{\circ} \mathrm{C}$ with stirring at $660 \mathrm{rpm}$ for $24 \mathrm{~h}$. The crude mixture was then directly adsorbed onto diatomaceous earth (Celite $\left.{ }^{\circledast}\right)$ and purified by flash column chromatography ( $20 \%$ ethyl acetate in hexanes). The product was obtained as an amber oil (32.0 $\mathrm{mg}, 62 \%)$.

$\mathrm{R}_{f}=0.20$ (20\% ethyl acetate in hexanes)

${ }^{1} \mathrm{H} \mathrm{NMR}\left(500 \mathrm{MHz}, \mathrm{CDCl}_{3}\right): \delta(\mathrm{ppm}) 7.28(\mathrm{t}, J=5.0 \mathrm{~Hz}, 2 \mathrm{H}), 7.04(\mathrm{~m}, 3 \mathrm{H}), 4.38(\mathrm{ddt}, J=10.7,8.9,4.8$ $\mathrm{Hz}, 1 \mathrm{H}), 2.60-2.46(\mathrm{~m}, 2 \mathrm{H}), 2.09-2.13(\mathrm{~m}, 1 \mathrm{H}), 2.10(\mathrm{~s}, 3 \mathrm{H}), 2.00-1.91(\mathrm{~m}, 1 \mathrm{H}), 1.87-1.78(\mathrm{~m}$, $1 \mathrm{H}), 1.72-1.66(\mathrm{~m}, 1 \mathrm{H}), 1.38(\mathrm{~s}, 3 \mathrm{H}), 1.33(\mathrm{~s}, 3 \mathrm{H})$

${ }^{13} \mathrm{C}$ NMR $\left(126 \mathrm{MHz}, \mathrm{CDCl}_{3}\right): \delta(\mathrm{ppm}) 207.91,168.23,147.59,128.64,123.45,122.97,78.42,44.48$, $41.71,39.69,30.22,29.63,26.71,26.66$

HRMS (ESI-TOF) $m / z:\left[\mathrm{M}+\mathrm{H}^{+}\right]$calculated for $\mathrm{C}_{16} \mathrm{H}_{22} \mathrm{NO}_{2}, 260.1651$; found, 260.1658

(Z)-(4,4-dimethyl-5-(phenylimino)tetrahydrofuran-2-yl)methyl acetate (2ah)

$\mathrm{PhN}$

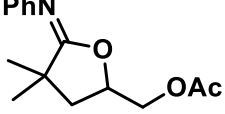

In a nitrogen-filled glove box, an oven-dried $4-\mathrm{mL}$ reaction vial was charged with $\mathrm{Cu}(\mathrm{OTf})_{2}(3.6 \mathrm{mg}, 10$ $\mu \mathrm{mol}, 5.0 \mathrm{~mol} \%), \mathrm{K}_{3} \mathrm{PO}_{4}(46.6 \mathrm{mg}, 0.220 \mathrm{mmol}, 1.10$ equiv), and a stir bar. Next, $400 \mu \mathrm{L}$ of a DCE stock solution containing 2,2'-bipyridine $(0.025 \mathrm{M}, 10 \mu \mathrm{mol}, 5.0 \mathrm{~mol} \%)$ was added. This was followed by addition of allyl acetate ( $108 \mu \mathrm{L}, 1.00 \mathrm{mmol}, 5.00$ equiv), aniline $(18.3 \mu \mathrm{L}, 0.200 \mathrm{mmol}, 1.00$ equiv $)$, and ethyl 2-bromo-2-methylpropanoate $(58.7 \mu \mathrm{L}, 0.400 \mathrm{mmol}, 2.00$ equiv). The vial was sealed with a Teflon-lined cap, removed from the glove box, and heated at $100{ }^{\circ} \mathrm{C}$ with stirring at $660 \mathrm{rpm}$ for $24 \mathrm{~h}$. The crude mixture was then directly adsorbed onto diatomaceous earth $\left(\mathrm{Celite}^{\circledR}\right)$ and purified by flash column chromatography (10-20\% ethyl acetate in hexanes). The product was obtained as a low melting solid (28.5 mg, 55\%).

$\mathrm{R}_{f}=0.35$ (20\% ethyl acetate in hexanes)

${ }^{1} \mathrm{H}$ NMR $\left(500 \mathrm{MHz}, \mathrm{CDCl}_{3}\right): \delta(\mathrm{ppm}) 7.32-7.22(\mathrm{~m}, 2 \mathrm{H}), 7.12-7.01(\mathrm{~m}, 3 \mathrm{H}), 4.67-4.55(\mathrm{~m}, 1 \mathrm{H})$, $4.26(\mathrm{dd}, J=12.1,3.4 \mathrm{~Hz}, 1 \mathrm{H}), 4.13(\mathrm{dd}, J=12.1,5.9 \mathrm{~Hz}, 1 \mathrm{H}), 2.12-2.08(\mathrm{~m}, 1 \mathrm{H}), 2.08(\mathrm{~s}, 3 \mathrm{H}), 1.84$ (dd, $J=12.5,10.1 \mathrm{~Hz}, 1 \mathrm{H}), 1.41(\mathrm{~s}, 3 \mathrm{H}), 1.36(\mathrm{~s}, 3 \mathrm{H})$

${ }^{13} \mathrm{C} \operatorname{NMR}\left(126 \mathrm{MHz}, \mathrm{CDCl}_{3}\right): \delta(\mathrm{ppm}) 170.97,167.71,147.10,128.76,123.81,123.02,76.51,65.53$, $41.38,40.41,26.93,26.65,21.05$

HRMS (ESI-TOF) $m / z:\left[\mathrm{M}+\mathrm{H}^{+}\right]$calculated for $\mathrm{C}_{15} \mathrm{H}_{20} \mathrm{NO}_{3}, 262.1443$; found, 262.1443

\section{(Z)-5-benzyl- $\mathrm{N}$-(4-methoxyphenyl)-3,3,5-trimethyldihydrofuran-2(3H)-imine (2ai)}

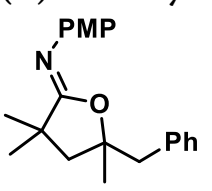

In a nitrogen-filled glove box, an oven-dried 4-mL reaction vial was charged with $\mathrm{Cu}(\mathrm{OTf})_{2}(3.6 \mathrm{mg}, 10$ $\mu \mathrm{mol}, 5.0 \mathrm{~mol} \%), \mathrm{K}_{3} \mathrm{PO}_{4}(46.6 \mathrm{mg}, 0.220 \mathrm{mmol}, 1.10$ equiv), and a stir bar. Next, $400 \mu \mathrm{L}$ of a DCE stock solution containing 2,2'-bipyridine (0.025 M, $10 \mu \mathrm{mol}, 5.0 \mathrm{~mol} \%)$ was added. This was followed by 
addition of (2-methallyl)benzene ( $89.9 \mu \mathrm{L}, 0.600 \mathrm{mmol}, 3.00$ equiv), 4-methoxyaniline ( $24.6 \mathrm{mg}, 0.200$ mmol, 1.00 equiv), and ethyl 2-bromo-2-methylpropanoate $(58.7 \mu \mathrm{L}, 0.400 \mathrm{mmol}, 2.00$ equiv). The vial was sealed with a Teflon-lined cap, removed from the glove box, and heated at $80^{\circ} \mathrm{C}$ with stirring at 660 $\mathrm{rpm}$ for $24 \mathrm{~h}$. The crude mixture was then directly adsorbed onto diatomaceous earth $\left(\mathrm{Celite}^{\otimes}\right)$ and purified by flash column chromatography (5-10\% ethyl acetate in hexanes). The product was obtained as a pale yellow oil $(22.7 \mathrm{mg}, 35 \%)$.

$\mathrm{R}_{f}=0.16$ ( $10 \%$ ethyl acetate in hexanes)

${ }^{1} \mathrm{H} \operatorname{NMR}\left(500 \mathrm{MHz}, \mathrm{CDCl}_{3}\right): \delta(\mathrm{ppm}) 7.32-7.21(\mathrm{~m}, 3 \mathrm{H}), 7.15(\mathrm{dd}, J=7.7,1.8 \mathrm{~Hz}, 2 \mathrm{H}), 7.10(\mathrm{~d}, J=$ $8.9 \mathrm{~Hz}, 2 \mathrm{H}), 6.86(\mathrm{~d}, J=8.9 \mathrm{~Hz}, 2 \mathrm{H}), 3.82(\mathrm{~s}, 3 \mathrm{H}), 2.96(\mathrm{~d}, J=13.8 \mathrm{~Hz}, 1 \mathrm{H}), 2.83(\mathrm{~d}, J=13.7 \mathrm{~Hz}, 1 \mathrm{H})$, $2.12(\mathrm{~d}, J=13.1 \mathrm{~Hz}, 1 \mathrm{H}), 1.85(\mathrm{~d}, J=13.1 \mathrm{~Hz}, 1 \mathrm{H}), 1.42(\mathrm{~s}, 3 \mathrm{H}), 1.39(\mathrm{~s}, 3 \mathrm{H}), 1.09(\mathrm{~s}, 3 \mathrm{H})$

${ }^{13} \mathrm{C}$ NMR $\left(126 \mathrm{MHz}, \mathrm{CDCl}_{3}\right): \delta(\mathrm{ppm}) 168.41,155.92,141.04,136.78,130.99,128.37,127.02,124.45$, $113.85,85.09,55.66,48.27,47.42,42.09,30.28,28.87,28.85$

HRMS (ESI-TOF) $m / z:\left[\mathrm{M}+\mathrm{H}^{+}\right]$calculated for $\mathrm{C}_{21} \mathrm{H}_{26} \mathrm{NO}_{2}$, 324.1964; found, 324.1951

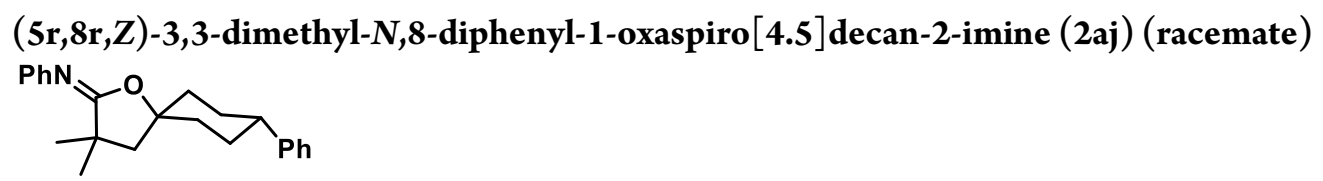

In a nitrogen-filled glove box, an oven-dried 4-mL reaction vial was charged with $\mathrm{Cu}(\mathrm{OTf})_{2}(1.8 \mathrm{mg}, 5.0$ $\mu \mathrm{mol}, 5.0 \mathrm{~mol} \%), \mathrm{K}_{3} \mathrm{PO}_{4}(23.3 \mathrm{mg}, 0.110 \mathrm{mmol}, 1.10$ equiv), and a stir bar. Next, $200 \mu \mathrm{L}$ of a DCE stock solution containing 2,2'-bipyridine $(0.025 \mathrm{M}, 5.0 \mu \mathrm{mol}, 5.0 \mathrm{~mol} \%)$. This was followed by addition of (4methylenecyclohexyl)benzene $(51.6 \mathrm{mg}, 0.300 \mathrm{mmol}, 3.00$ equiv), aniline $(9.10 \mu \mathrm{L}, 0.100 \mathrm{mmol}, 1.00$ equiv), and ethyl 2-bromo-2-methylpropanoate $(29.4 \mu \mathrm{L}, 0.200 \mathrm{mmol}, 2.00$ equiv). The vial was sealed with a Teflon-lined cap, removed from the glove box, and heated at $100^{\circ} \mathrm{C}$ with stirring at $660 \mathrm{rpm}$ for 24 $\mathrm{h}$. The crude mixture was then directly adsorbed onto diatomaceous earth (Celite ${ }^{\circledast}$ ) and purified by flash column chromatography ( $5 \%$ ethyl acetate in hexanes). The product was obtained as a colorless oil (25.8 $\mathrm{mg}, 77 \%, 10: 1$ d.r.). For determination of relative stereochemistry, please refer to Page S47.

$\mathrm{R}_{f}=0.20$ (5\% ethyl acetate in hexanes)

${ }^{1} \mathrm{H}$ NMR $\left(500 \mathrm{MHz}, \mathrm{CDCl}_{3}\right): \delta(\mathrm{ppm}) 7.32-7.23(\mathrm{~m}, 4 \mathrm{H}), 7.22-7.15(\mathrm{~m}, 3 \mathrm{H}), 7.12-7.05(\mathrm{~m}, 2 \mathrm{H})$, $7.04-6.99(\mathrm{~m}, 1 \mathrm{H}), 2.51(\mathrm{dddd}, J=11.9,11.9,3.3,3.3 \mathrm{~Hz}, 1 \mathrm{H}), 2.08(\mathrm{~s}, 2 \mathrm{H}), 1.98-1.91(\mathrm{~m}, 3 \mathrm{H}), 1.90$ $-1.82(\mathrm{~m}, 3 \mathrm{H}), 1.60-1.49(\mathrm{~m}, 2 \mathrm{H}), 1.44(\mathrm{~s}, 6 \mathrm{H})$

${ }^{13} \mathrm{C} \mathrm{NMR}\left(126 \mathrm{MHz}, \mathrm{CDCl}_{3}\right): \delta(\mathrm{ppm}) 168.93,146.04,128.73,128.68,126.98,126.56,123.64,123.39$, $123.17,50.33,46.74,43.04,38.90,31.42,30.40,30.03$

HRMS (ESI-TOF) $m / z:\left[\mathrm{M}+\mathrm{H}^{+}\right]$calculated for $\mathrm{C}_{23} \mathrm{H}_{28} \mathrm{NO}, 334.2171$; found, 334.2168

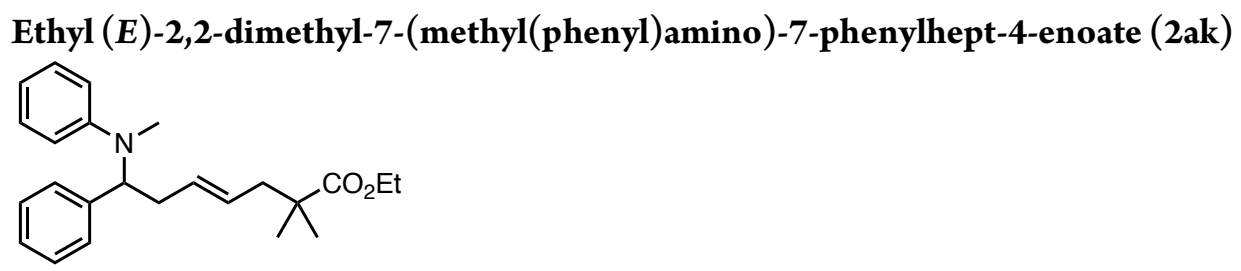


In a nitrogen-filled glove box, an oven-dried 4-mL reaction vial was charged with $\mathrm{Cu}(\mathrm{OTf})_{2}(3.6 \mathrm{mg}, 10$ $\mu \mathrm{mol}, 5.0 \mathrm{~mol} \%), \mathrm{K}_{3} \mathrm{PO}_{4}(46.6 \mathrm{mg}, 0.220 \mathrm{mmol}, 1.10$ equiv), and a stir bar. Next, $400 \mu \mathrm{L}$ of a DCE stock solution containing 2,2'-bipyridine $(0.025 \mathrm{M}, 10 \mu \mathrm{mol}, 5.0 \mathrm{~mol} \%)$ was added. This was followed by addition of trans-(2-vinylcyclopropyl)benzene ( $31.0 \mu \mathrm{L}, 0.200 \mathrm{mmol}, 1.00$ equiv), $N$-methylaniline (22.0 $\mu \mathrm{L}, 0.200 \mathrm{mmol}, 1.00$ equiv), 1.0 equiv), and ethyl 2-bromo-2-methylpropanoate ( $58.7 \mu \mathrm{L}, 0.400 \mathrm{mmol}$, 2.00 equiv). The vial was sealed with a Teflon-lined cap, removed from the glove box, and heated at $80^{\circ} \mathrm{C}$ with stirring at $660 \mathrm{rpm}$ for $24 \mathrm{~h}$. The crude mixture was then directly adsorbed onto diatomaceous earth $\left(\right.$ Celite $\left.{ }^{\oplus}\right)$ and purified by flash column chromatography (3\% ethyl acetate in hexanes). The product was obtained as a colorless oil ( $30.3 \mathrm{mg}, 42 \%)$.

$\mathrm{R}_{f}=0.25$ (2.5\% ethyl acetate in hexanes)

${ }^{1} \mathrm{H}$ NMR $\left(500 \mathrm{MHz}, \mathrm{CDCl}_{3}\right): \delta(\mathrm{ppm}) 7.33-7.28(\mathrm{~m}, 2 \mathrm{H}), 7.28-7.20(\mathrm{~m}, 5 \mathrm{H}), 6.81(\mathrm{~d}, J=8.0 \mathrm{~Hz}$, $2 \mathrm{H}), 6.71(\mathrm{t}, J=7.3 \mathrm{~Hz}, 1 \mathrm{H}), 5.51-5.44(\mathrm{~m}, 2 \mathrm{H}), 5.01(\mathrm{dd}, J=8.9,6.4 \mathrm{~Hz}, 1 \mathrm{H}), 4.05(\mathrm{q}, J=7.1 \mathrm{~Hz}$, $2 \mathrm{H}), 2.82-2.75(\mathrm{~m}, 1 \mathrm{H}), 2.71(\mathrm{~s}, 3 \mathrm{H}), 2.69-2.65(\mathrm{~m}, 1 \mathrm{H}), 2.19-2.14(\mathrm{~m}, 2 \mathrm{H}), 1.20(\mathrm{t}, J=7.1 \mathrm{~Hz}$, $3 \mathrm{H}), 1.08(\mathrm{~s}, 6 \mathrm{H})$

${ }^{13} \mathrm{C} \mathrm{NMR}\left(126 \mathrm{MHz}, \mathrm{CDCl}_{3}\right): \delta(\mathrm{ppm}) 177.83,150.79,141.43,130.89,129.39,128.62,128.40,127.51$, $127.22,116.83,113.29,61.90,60.49,43.74,42.58,35.29,32.17,25.04,25.02,14.51$

HRMS (ESI-TOF) $m / z:\left[\mathrm{M}+\mathrm{H}^{+}\right]$calculated for $\mathrm{C}_{24} \mathrm{H}_{32} \mathrm{NO}_{2}, 366.2433$; found, 366.2445

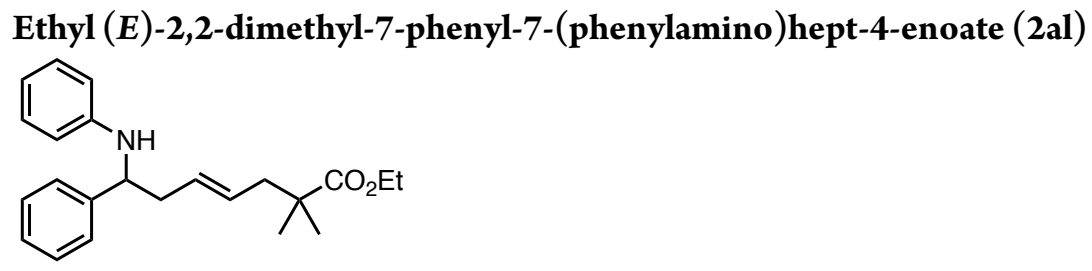

In a nitrogen-filled glove box, an oven-dried $4-\mathrm{mL}$ reaction vial was charged with $\mathrm{Cu}(\mathrm{OTf})_{2}(3.6 \mathrm{mg}, 10$ $\mu \mathrm{mol}, 5.0 \mathrm{~mol} \%), \mathrm{K}_{3} \mathrm{PO}_{4}(46.6 \mathrm{mg}, 0.220 \mathrm{mmol}, 1.10$ equiv), and a stir bar. Next, $400 \mu \mathrm{L}$ of a DCE stock solution containing 2,2'-bipyridine $(0.025 \mathrm{M}, 10 \mu \mathrm{mol}, 5.0 \mathrm{~mol} \%)$ was added. This was followed by addition of trans-(2-vinylcyclopropyl)benzene ( $31.0 \mu \mathrm{L}, 0.200 \mathrm{mmol}, 1.00$ equiv), aniline (18.3 $\mu \mathrm{L}, 0.200$ mmol, 1.00 equiv), and ethyl 2-bromo-2-methylpropanoate $(58.7 \mu \mathrm{L}, 0.400 \mathrm{mmol}, 2.00$ equiv). The vial was sealed with a Teflon-lined cap, removed from the glove box, and heated at $80^{\circ} \mathrm{C}$ with stirring at 660 rpm for $24 \mathrm{~h}$. The crude mixture was then directly adsorbed onto diatomaceous earth $\left(\right.$ Celite $\left.^{\circledast}\right)$ and purified by flash column chromatography ( $2.5 \%$ ethyl acetate in hexanes). The product was obtained as a colorless oil ( $35.2 \mathrm{mg}, 50 \%)$.

$\mathrm{R}_{f}=0.20$ (2.5\% ethyl acetate in hexanes)

${ }^{1} \mathrm{H}$ NMR $\left(500 \mathrm{MHz}, \mathrm{CDCl}_{3}\right): \delta(\mathrm{ppm}) 7.38-7.28(\mathrm{~m}, 4 \mathrm{H}), 7.24-7.19(\mathrm{~m}, 1 \mathrm{H}), 7.12-7.02(\mathrm{~m}, 2 \mathrm{H})$, $6.68-6.60(\mathrm{~m}, 1 \mathrm{H}), 6.56-6.43(\mathrm{~m}, 2 \mathrm{H}), 5.53(\mathrm{ddd}, J=14.0,7.5,6.5 \mathrm{~Hz}, 1 \mathrm{H}), 5.38$ (ddd, $J=14.2,7.4$, $6.7 \mathrm{~Hz}, 1 \mathrm{H}), 4.35(\mathrm{dd}, J=7.8,5.0 \mathrm{~Hz}, 1 \mathrm{H}), 4.23(\mathrm{~s}, 1 \mathrm{H}), 4.11(\mathrm{qd}, J=7.1,1.5 \mathrm{~Hz}, 2 \mathrm{H}), 2.61-2.52(\mathrm{~m}$, $1 \mathrm{H}), 2.45(\mathrm{ddd}, J=14.5,7.7,6.7 \mathrm{~Hz}, 1 \mathrm{H}), 2.29-2.21(\mathrm{~m}, 2 \mathrm{H}), 1.22(\mathrm{t}, J=7.1 \mathrm{~Hz}, 3 \mathrm{H}), 1.17(\mathrm{~s}, 6 \mathrm{H})$

${ }^{13} \mathrm{C}$ NMR $\left(126 \mathrm{MHz}, \mathrm{CDCl}_{3}\right): \delta$ (ppm) 177.69, 147.65, 143.94, 130.34, 129.56, 129.28, 128.82, 127.14, $126.55,117.45,113.73,60.67,57.33,43.92,42.76,42.45,25.31,25.06,14.55$

HRMS (ESI-TOF) $m / z:\left[\mathrm{M}+\mathrm{H}^{+}\right]$calculated for $\mathrm{C}_{23} \mathrm{H}_{30} \mathrm{NO}_{2}, 352.2277$; found, 352.2276 
Ethyl 2-methyl-2-(1-(methyl(phenyl)amino)-2,3-dihydro-1H-inden-2-yl)propanoate (2am) (racemate)<smiles>CCOC(=O)C(C)(C)C1Cc2ccccc2C1N(C)c1ccccc1</smiles>

In a nitrogen-filled glove box, an oven-dried 4-mL reaction vial was charged with $\mathrm{Cu}(\mathrm{OTf})_{2}(3.6 \mathrm{mg}, 10$ $\mu \mathrm{mol}, 5.0 \mathrm{~mol} \%), \mathrm{K}_{3} \mathrm{PO}_{4}(46.6 \mathrm{mg}, 0.220 \mathrm{mmol}, 1.10$ equiv), and a stir bar. Next, $400 \mu \mathrm{L}$ of a DCE stock solution containing 2,2'-bipyridine $(0.025 \mathrm{M}, 10 \mu \mathrm{mol}, 5.0 \mathrm{~mol} \%)$ was added. This was followed by addition of indene $(23.3 \mu \mathrm{L}, 0.200 \mathrm{mmol}, 1.00$ equiv), $N$-methylaniline $(22.4 \mu \mathrm{L}, 0.200 \mathrm{mmol}, 1.00$ equiv), and ethyl 2-bromo-2-methylpropanoate ( $58.7 \mu \mathrm{L}, 0.400 \mathrm{mmol}, 2.00$ equiv). The vial was sealed with a Teflon-lined cap, removed from the glove box, and heated at $80^{\circ} \mathrm{C}$ with stirring at $660 \mathrm{rpm}$ for 24 $\mathrm{h}$. The crude mixture was then directly adsorbed onto diatomaceous earth (Celite ${ }^{\circledast}$ ) and purified by flash column chromatography (2.5\% ethyl acetate in hexanes). The product was obtained as a colorless oil ( $37.7 \mathrm{mg}, 56 \%,>20: 1$ d.r.). It was determined to be the trans isomer based on coupling constant analysis. See: Janody, S.; Jazzar, R.; Comte, A.; Holstein, P. M.; Vors, J-. P.; Ford, M. J.; Baudoin, O. Chem. Eur. J. 2014, 20, 11084-11090.

$\mathrm{R}_{f}=0.32$ (5\% ethyl acetate in hexanes)

Crude D.R. (GC): 50:1

${ }^{1} \mathrm{H}$ NMR (500 MHz, $\left.\mathrm{CDCl}_{3}\right): \delta(\mathrm{ppm}) 7.25(\mathrm{~m}, 2 \mathrm{H}), 7.20(\mathrm{~m}, 2 \mathrm{H}), 7.12(\mathrm{~m}, 1 \mathrm{H}), 7.03(\mathrm{~d}, 1 \mathrm{H}), 6.89$ $(\mathrm{m}, 2 \mathrm{H}), 6.72(\mathrm{~m}, 1 \mathrm{H}), 5.52(\mathrm{~d}, J=8.5 \mathrm{~Hz}, 1 \mathrm{H}), 3.94(\mathrm{dq}, J=10.8,7.1 \mathrm{~Hz}, 1 \mathrm{H}), 3.88(\mathrm{dq}, J=10.8,7.1$ $\mathrm{Hz}, 1 \mathrm{H}), 2.66(\mathrm{~s}, 3 \mathrm{H}), 3.02(\mathrm{dd}, J=15.1,8.7 \mathrm{~Hz}, 1 \mathrm{H}), 2.95(\mathrm{dd}, J=8.6,8.6 \mathrm{~Hz}, 1 \mathrm{H}), 2.78(\mathrm{dd}, J=15.1$, $8.9 \mathrm{~Hz}, 1 \mathrm{H}), 1.22(\mathrm{~s}, 3 \mathrm{H}), 1.22(\mathrm{~s}, 3 \mathrm{H}), 1.09(\mathrm{t}, J=7.1 \mathrm{~Hz}, 3 \mathrm{H})$

${ }^{13} \mathrm{C} \mathrm{NMR}\left(125 \mathrm{MHz}, \mathrm{CDCl}_{3}\right): \delta$ (ppm) 177.49, 150.70, 142.87, 141.61, 129.50, 127.90, 126.84, 124.99, $124.23,116.88,113.26,65.12,60.88,51.23,44.05,32.83,32.77,24.10,22.95,14.25$

HRMS (ESI-TOF) $m / z:\left[\mathrm{M}+\mathrm{H}^{+}\right]$calculated for $\mathrm{C}_{22} \mathrm{H}_{28} \mathrm{NO}_{2}$, 338.2120; found, 338.2117

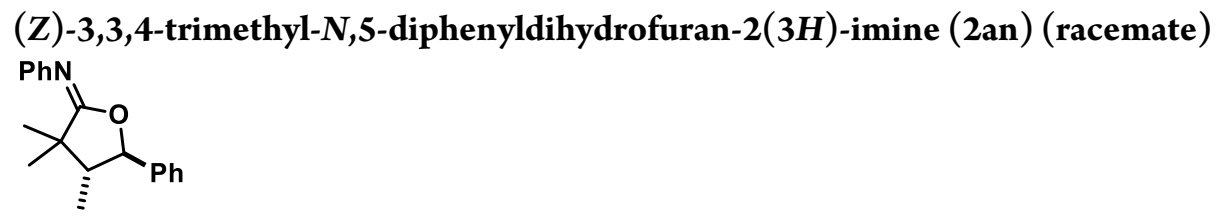

In a nitrogen-filled glove box, an oven-dried 4-mL reaction vial was charged with $\mathrm{Cu}(\mathrm{OTf})_{2}(3.6 \mathrm{mg}, 10$ $\mu \mathrm{mol}, 5.0 \mathrm{~mol} \%), \mathrm{K}_{3} \mathrm{PO}_{4}(46.6 \mathrm{mg}, 0.220 \mathrm{mmol}, 1.10$ equiv), and a stir bar. Next, $400 \mu \mathrm{L}$ of a DCE stock solution containing 2,2'-bipyridine $(0.025 \mathrm{M}, 10 \mu \mathrm{mol}, 5.0 \mathrm{~mol} \%)$ was added. This was followed by addition of cis- $\beta$-methylstyrene $(77.6 \mu \mathrm{L}, 0.600 \mathrm{mmol}, 3.00$ equiv), aniline $(18.3 \mu \mathrm{L}, 0.200 \mathrm{mmol}, 1.00$ equiv), and ethyl 2-bromo-2-methylpropanoate $(88.1 \mu \mathrm{L}, 0.600 \mathrm{mmol}, 3.00$ equiv). The vial was sealed with a Teflon-lined cap, removed from the glove box, and heated at $100^{\circ} \mathrm{C}$ with stirring at $660 \mathrm{rpm}$ for 24 $\mathrm{h}$. The crude mixture was then directly adsorbed onto diatomaceous earth (Celite ${ }^{\oplus}$ ) and purified by flash column chromatography (4-7.5\% ethyl acetate in hexanes). The product was obtained as a white solid, which contained $10 \%$ lactone as an impurity $(45.2 \mathrm{mg}, 73 \%,>20: 1$ d.r.). It was determined to be the cis isomer based on coupling constant analysis. See: Fang, J-. M.; Chen, S-. T.; Chen, I-. H. J. Organomet. Chem. 1990, 398, 219-224. 
$\mathrm{R}_{f}=0.27$ (5\% ethyl acetate in hexanes)

Crude D.R. (GC): 25:1

$\mathrm{mp}: 72-78^{\circ} \mathrm{C}$

Peaks for the lactone impurity are denoted by \{\}

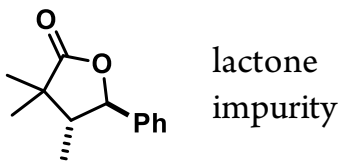

${ }^{1} \mathrm{H} \operatorname{NMR}\left(500 \mathrm{MHz}, \mathrm{CDCl}_{3}\right): \delta(\mathrm{ppm}) 7.40-7.32(\mathrm{~m}, 3 \mathrm{H}), 7.32-7.25(\mathrm{~m}, 4 \mathrm{H}), 7.17-7.07(\mathrm{~m}, 2 \mathrm{H})$, $7.06-6.99(\mathrm{~m}, 1 \mathrm{H}),\{4.88(\mathrm{~d}, J=10.3 \mathrm{~Hz}, 0.10 \mathrm{H})\}, 4.83(\mathrm{~d}, J=10.3 \mathrm{~Hz}, 1 \mathrm{H}),\{2.16-2.07(\mathrm{~m}, 0.10 \mathrm{H})\}$, $2.05(\mathrm{dq}, J=10.4,6.8 \mathrm{~Hz}, 1 \mathrm{H}), 1.37(\mathrm{~s}, 3 \mathrm{H}), 1.26(\mathrm{~s}, 3 \mathrm{H}), 0.99(\mathrm{~d}, J=6.8 \mathrm{~Hz}, 3 \mathrm{H})$

${ }^{13} \mathrm{C} \mathrm{NMR}\left(126 \mathrm{MHz}, \mathrm{CDCl}_{3}\right): \delta(\mathrm{ppm}) 168.54,147.39,139.07,128.90,128.78,128.65,126.47,123.63$, $122.99,86.70,51.10,44.17,24.82,20.66,9.62$

HRMS (ESI-TOF) $m / z:\left[\mathrm{M}+\mathrm{H}^{+}\right]$calculated for $\mathrm{C}_{19} \mathrm{H}_{22} \mathrm{NO}, 280.1701$; found, 280.1696

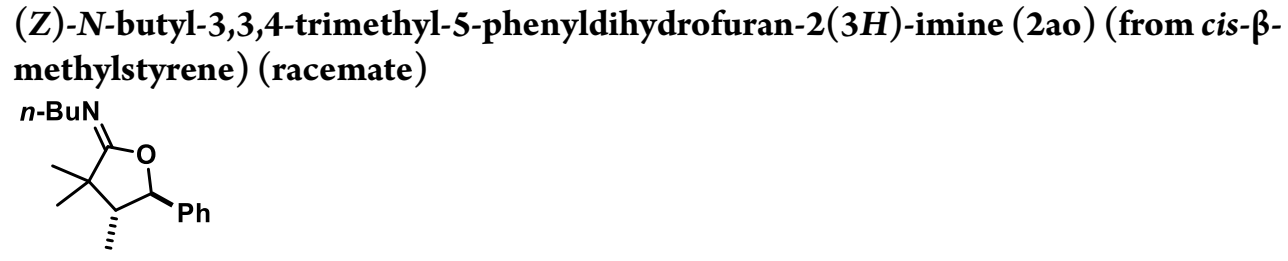

In a nitrogen-filled glove box, an oven-dried 4-mL reaction vial was charged with $\mathrm{Cu}(\mathrm{OTf})_{2}(1.8 \mathrm{mg}, 5.0$ $\mu \mathrm{mol}, 5.0 \mathrm{~mol} \%), \mathrm{K}_{3} \mathrm{PO}_{4}(23.3 \mathrm{mg}, 0.110 \mathrm{mmol}, 1.10$ equiv), and a stir bar. Next, $200 \mu \mathrm{L}$ of a DCE stock solution containing 2,2'-bipyridine $(0.025 \mathrm{M}, 5.0 \mu \mathrm{mol}, 5.0 \mathrm{~mol} \%)$ was added. This was followed by addition of cis- $\beta$-methylstyrene $(38.8 \mu \mathrm{L}, 0.300 \mathrm{mmol}, 3.00$ equiv), butylamine $(9.90 \mu \mathrm{L}, 0.100 \mathrm{mmol}$, 1.00 equiv), and ethyl 2-bromo-2-methylpropanoate ( $44.1 \mu \mathrm{L}, 0.300 \mathrm{mmol}, 3.00$ equiv). The vial was sealed with a Teflon-lined cap, removed from the glove box, and heated at $100^{\circ} \mathrm{C}$ with stirring at $660 \mathrm{rpm}$ for $24 \mathrm{~h}$. The crude mixture was then directly adsorbed onto diatomaceous earth (Celite ${ }^{\oplus}$ ) and purified by flash column chromatography ( $10 \%$ ethyl acetate in hexanes). The product was obtained as a colorless oil ( $12.5 \mathrm{mg}, 48 \%$, > $20: 1$ d.r.).

$\mathrm{R}_{f}=0.30$ ( $10 \%$ ethyl acetate in hexanes)

Crude D.R. (GC): 32:1

${ }^{1} \mathrm{H} \mathrm{NMR}\left(500 \mathrm{MHz}, \mathrm{CDCl}_{3}\right): \delta(\mathrm{ppm}) 7.41-7.29(\mathrm{~m}, 5 \mathrm{H}), 4.72(\mathrm{~d}, J=10.3 \mathrm{~Hz}, 1 \mathrm{H}), 3.35(\mathrm{dt}, J=12.4$, $7.3 \mathrm{~Hz}, 1 \mathrm{H}), 3.30(\mathrm{dt}, J=12.4,7.2 \mathrm{~Hz}, 1 \mathrm{H}), 1.90(\mathrm{dq}, J=10.3,6.8 \mathrm{~Hz}, 1 \mathrm{H}), 1.55(\mathrm{dtd}, J=14.6,7.3,3.2$ $\mathrm{Hz}, 2 \mathrm{H}), 1.40-1.29(\mathrm{~m}, 2 \mathrm{H}), 1.21(\mathrm{~s}, 3 \mathrm{H}), 1.11(\mathrm{~s}, 3 \mathrm{H}), 0.96-0.87(\mathrm{~m}, 6 \mathrm{H})$

${ }^{13} \mathrm{C}$ NMR $\left(126 \mathrm{MHz}, \mathrm{CDCl}_{3}\right): \delta(\mathrm{ppm}) 168.14,139.82,128.79,128.57,126.53,85.51,51.40,46.94$, $43.22,33.37,24.85,20.91,20.68,14.35,9.58$

HRMS (ESI-TOF) $m / z:\left[\mathrm{M}+\mathrm{H}^{+}\right]$calculated for $\mathrm{C}_{17} \mathrm{H}_{26} \mathrm{NO}, 260.2014$; found, 260.2015 
(Z)-N-butyl-3,3,4-trimethyl-5-phenyldihydrofuran-2(3H)-imine (2ao) (from trans- $\beta$ methylstyrene) (racemate)

n-BuN<smiles>C[C@H]1C(c2ccccc2)OC(=N[SeH])C1(C)C</smiles>

In a nitrogen-filled glove box, an oven-dried 4-mL reaction vial was charged with $\mathrm{Cu}(\mathrm{OTf})_{2}(1.8 \mathrm{mg}, 5.0$ $\mu \mathrm{mol}, 5.0 \mathrm{~mol} \%), \mathrm{K}_{3} \mathrm{PO}_{4}(23.3 \mathrm{mg}, 0.110 \mathrm{mmol}, 1.10$ equiv), and a stir bar. Next, $200 \mu \mathrm{L}$ of a DCE stock solution containing $2,2^{\prime}$-bipyridine $(0.025 \mathrm{M}, 5.0 \mu \mathrm{mol}, 5.0 \mathrm{~mol} \%)$ was added. This was followed by addition of trans- $\beta$-methylstyrene $(38.9 \mu \mathrm{L}, 0.300 \mathrm{mmol}, 3.00$ equiv $)$, butylamine $(9.90 \mu \mathrm{L}, 0.100 \mathrm{mmol}$, 1.00 equiv), and ethyl 2-bromo-2-methylpropanoate ( $44.1 \mu \mathrm{L}, 0.300 \mathrm{mmol}, 3.00$ equiv). The vial was sealed with a Teflon-lined cap, removed from the glove box, and heated at $100{ }^{\circ} \mathrm{C}$ with stirring at $660 \mathrm{rpm}$ for $24 \mathrm{~h}$. The crude mixture was then directly adsorbed onto diatomaceous earth (Celite ${ }^{\circledR}$ ) and purified by flash column chromatography ( $10 \%$ ethyl acetate in hexanes). The product was obtained as a colorless oil ( $14.5 \mathrm{mg}, 56 \%,>20: 1$ d.r.).

$\mathrm{R}_{f}=0.30$ (10\% ethyl acetate in hexanes)

Crude D.R. (GC): 24:1

${ }^{1} \mathrm{H}$ NMR $\left(500 \mathrm{MHz}, \mathrm{CDCl}_{3}\right): \delta(\mathrm{ppm}) 7.41-7.29(\mathrm{~m}, 5 \mathrm{H}), 4.72(\mathrm{~d}, J=10.3 \mathrm{~Hz}, 1 \mathrm{H}), 3.35(\mathrm{dt}, J=12.4$, $7.3 \mathrm{~Hz}, 1 \mathrm{H}), 3.30(\mathrm{dt}, J=12.4,7.2 \mathrm{~Hz}, 1 \mathrm{H}), 1.90(\mathrm{dq}, J=10.3,6.8 \mathrm{~Hz}, 1 \mathrm{H}), 1.55$ (dtd, $J=14.6,7.3,3.2$

$\mathrm{Hz}, 2 \mathrm{H}), 1.40-1.29(\mathrm{~m}, 2 \mathrm{H}), 1.21(\mathrm{~s}, 3 \mathrm{H}), 1.11(\mathrm{~s}, 3 \mathrm{H}), 0.96-0.87(\mathrm{~m}, 6 \mathrm{H})$

${ }^{13} \mathrm{C} \mathrm{NMR}\left(126 \mathrm{MHz}, \mathrm{CDCl}_{3}\right): \delta(\mathrm{ppm}) 168.14,139.82,128.79,128.57,126.53,85.51,51.40,46.94$, $43.22,33.37,24.85,20.91,20.68,14.35,9.58$

HRMS (ESI-TOF) $m / z:\left[\mathrm{M}+\mathrm{H}^{+}\right]$calculated for $\mathrm{C}_{17} \mathrm{H}_{26} \mathrm{NO}, 260.2014$; found, 260.2003

Ethyl 3-(4-(bromomethyl)-1-phenylpyrrolidin-3-yl)-2,2-dimethylpropanoate (2ap)<smiles>CCOCC(C)(C)CC1CN(c2ccccc2)CC1CBr</smiles>

In a nitrogen-filled glove box, an oven-dried 4-mL reaction vial was charged with $\mathrm{Cu}(\mathrm{OTf})_{2}(3.6 \mathrm{mg}, 10$ $\mu \mathrm{mol}, 5.0 \mathrm{~mol} \%), \mathrm{K}_{3} \mathrm{PO}_{4}(46.6 \mathrm{mg}, 0.220 \mathrm{mmol}, 1.10$ equiv), and a stir bar. Next, $400 \mu \mathrm{L}$ of a DCE stock solution containing 2,2'-bipyridine $(0.025 \mathrm{M}, 10 \mu \mathrm{mol}, 5.0 \mathrm{~mol} \%)$ was added. This was followed by addition of $N, N$-diallylaniline $(36.7 \mu \mathrm{L}, 0.200 \mathrm{mmol}, 1.00$ equiv), aniline $(18.3 \mu \mathrm{L}, 0.200 \mathrm{mmol}, 1.00$ equiv), and ethyl 2-bromo-2-methylpropanoate $(58.7 \mu \mathrm{L}, 0.400 \mathrm{mmol}, 2.00$ equiv). The vial was sealed with a Teflon-lined cap, removed from the glove box, and heated at $100^{\circ} \mathrm{C}$ with stirring at $660 \mathrm{rpm}$ for 24 $\mathrm{h}$. The crude mixture was then directly adsorbed onto diatomaceous earth $\left(\mathrm{Celite}^{\circledast}\right)$ and purified by flash column chromatography ( $3 \%$ ethyl acetate in hexanes) to produce both product diastereomers as a yellow oil (50.8 mg, 69\%).

$\mathrm{R}_{f}=0.20$ (3\% ethyl acetate in hexanes)

Diastereomer 1: ${ }^{1} \mathrm{H}$ NMR $\left(500 \mathrm{MHz}, \mathrm{CDCl}_{3}\right): \delta(\mathrm{ppm}) 7.23(\mathrm{dd}, J=8.6,7.2 \mathrm{~Hz}, 2 \mathrm{H}), 6.70(\mathrm{t}, J=7.3 \mathrm{~Hz}$, $1 \mathrm{H}), 6.55(\mathrm{~d}, J=8.1 \mathrm{~Hz}, 2 \mathrm{H}), 4.19-4.11(\mathrm{~m}, 2 \mathrm{H}), 3.61(\mathrm{dd}, J=10.0,3.7 \mathrm{~Hz}, 1 \mathrm{H}), 3.49(\mathrm{dd}, J=9.7,2.9$ $\mathrm{Hz}, 1 \mathrm{H}), 3.40(\mathrm{dd}, J=9.7,5.9 \mathrm{~Hz}, 1 \mathrm{H}), 3.36(\mathrm{dd}, J=9.2,7.7 \mathrm{~Hz}, 1 \mathrm{H}), 3.29(\mathrm{t}, J=10.5 \mathrm{~Hz}, 1 \mathrm{H}), 3.04(\mathrm{t}, J$ 
$=9.0 \mathrm{~Hz}, 1 \mathrm{H}), 2.66(\mathrm{dtdd}, J=10.5,6.1,4.1,2.9 \mathrm{~Hz}, 1 \mathrm{H}), 2.49-2.39(\mathrm{~m}, 1 \mathrm{H}), 1.84(\mathrm{dd}, J=14.0,4.2 \mathrm{~Hz}$, $1 \mathrm{H}), 1.67(\mathrm{dd}, J=14.0,8.8 \mathrm{~Hz}, 1 \mathrm{H}), 1.27(\mathrm{t}, J=7.1 \mathrm{~Hz}, 3 \mathrm{H}), 1.24(\mathrm{~s}, 6 \mathrm{H})$

Diastereomer 1: ${ }^{13} \mathrm{C}$ NMR $(126 \mathrm{MHz}, \mathrm{CDCl} 3) \delta 177.78,147.55,129.49,111.79,77.30,61.05,52.57$, $51.94,45.55,42.26,39.16,38.89,32.98,26.36,25.72,14.47$

Diastereomer 2: ${ }^{1} \mathrm{H}$ NMR $\left(500 \mathrm{MHz}, \mathrm{CDCl}_{3}\right): \delta(\mathrm{ppm}) 7.23(\mathrm{dd}, J=8.7,7.1 \mathrm{~Hz}, 2 \mathrm{H}), 6.75-6.66(\mathrm{~m}$, $1 \mathrm{H}), 6.60-6.51(\mathrm{~m}, 2 \mathrm{H}), 4.16(\mathrm{q}, J=7.1 \mathrm{~Hz}, 2 \mathrm{H}), 3.64(\mathrm{dd}, J=10.3,3.9 \mathrm{~Hz}, 1 \mathrm{H}), 3.61(\mathrm{dd}, J=9.2,7.6$ $\mathrm{Hz}, 1 \mathrm{H}), 3.57-3.53(\mathrm{~m}, 1 \mathrm{H}), 3.40(\mathrm{dd}, J=10.3,8.2 \mathrm{~Hz}, 1 \mathrm{H}), 3.18(\mathrm{t}, J=8.8 \mathrm{~Hz}, 1 \mathrm{H}), 3.06-2.97(\mathrm{~m}$, $1 \mathrm{H}), 2.39-2.30(\mathrm{~m}, 1 \mathrm{H}), 2.15-2.04(\mathrm{~m}, 1 \mathrm{H}), 1.90(\mathrm{dd}, J=14.1,2.5 \mathrm{~Hz}, 1 \mathrm{H}), 1.67(\mathrm{dd}, J=14.1,9.6 \mathrm{~Hz}$, $1 \mathrm{H}), 1.29(\mathrm{t}, J=7.1 \mathrm{~Hz}, 3 \mathrm{H}), 1.24(\mathrm{~s}, 3 \mathrm{H}), 1.24(\mathrm{~s}, 3 \mathrm{H})$

Diastereomer 2: ${ }^{13} \mathrm{C}$ NMR (126 MHz, CDCl3) $\delta 177.81,129.50,112.17,108.04,77.30,61.01,47.21$, $44.10,42.45,39.93,39.18,38.90,34.59,26.12,26.07,14.52$

HRMS (ESI-TOF) $m / z$ : $\left[\mathrm{M}+\mathrm{H}^{+}\right]$calculated for $\mathrm{C}_{18} \mathrm{H}_{27} \mathrm{NO}_{2} \mathrm{Br}$, 368.1218; found, 368.1225

\section{F. Procedure for Large Scale Carboamination Reaction: 10 mmol scale: Ethyl 2,2-dimethyl-4-(methyl(phenyl)amino)-4-phenylbutanoate (2a) \\ $\mathrm{Ph}$.<smiles>CCOC(=O)CC(C)(C)CC(NC)c1ccccc1</smiles>

In a glovebox, an oven dried 100-mL bomb containing a football shaped magnetic stir bar was charged with $\mathrm{K}_{3} \mathrm{PO}_{4}\left(2.33 \mathrm{~g}, 11.0 \mathrm{mmol}, 1.10\right.$ equiv), $\mathrm{Cu}(\mathrm{OTf})_{2}(180.8 \mathrm{mg}, 0.500 \mathrm{mmol}, 5.00 \mathrm{~mol} \%)$, and 2,2 bipyridyl $(78.1 \mathrm{mg}, 0.500 \mathrm{mmol}, 5.00 \mathrm{~mol} \%)$. 1,2-dichloroethane $(20.0 \mathrm{~mL})$ was then added to the bomb, followed by styrene $(1.15 \mathrm{~mL}, 10.0 \mathrm{mmol}, 1.00$ equiv), $N$-methylaniline $(1.08 \mathrm{~mL}, 10.0 \mathrm{mmol}$, 1.00 equiv), and ethyl 2-bromo-2-methylpropanoate $(2.93 \mathrm{~mL}, 20.0 \mathrm{mmol}, 2.00$ equiv). The bomb was sealed with a screw cap, removed from the glovebox, and heated at $80^{\circ} \mathrm{C}$ for 24 hours. The contents of the bomb were diluted with dichloromethane and filtered through a pad of Celite (height $=2 \mathrm{~cm}$, diameter $=7 \mathrm{~cm}$ ), which was washed several times with dichloromethane. The resultant purple liquid was combined with diatomaceous earth $\left(\mathrm{Celite}^{\circledast}\right)$ in a 250 -mL round bottom flask, concentrated by rotary evaporation to yield a dry powder, and loaded directly onto a column of silica gel for flash column chromatography (2.5\% ethyl acetate in hexanes). The product fractions were collected and the residue was subjected to flash column chromatography (2.5\% ethyl acetate in hexanes) a second time, which yields the product as a pale-yellow oil (3.076 g, 95\% yield). Characterization data was identical to that reported above.

\section{G. Deprotection of $2 q$ to form the N-H Lactam}

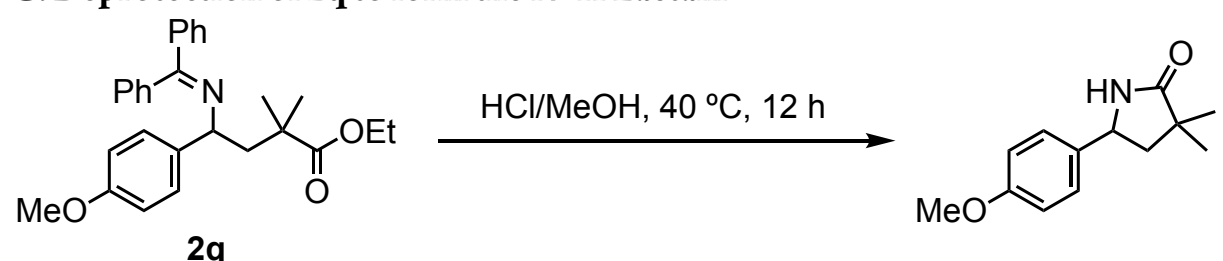

The crude reaction to form $\mathbf{2 q}$ was charged with concentrated $\mathrm{HCl}(\sim 2 \mathrm{~mL})$ and $\mathrm{MeOH}(\sim 2 \mathrm{~mL})$ and was stirred at $40{ }^{\circ} \mathrm{C}$ overnight $(12 \mathrm{~h})$. The mixture was then cooled and basified with $\mathrm{NaOH}(\mathrm{aq}, 5 \mathrm{M})$. GC-MS analysis (shown below) of the organic layer indicated complete conversion to the $\mathrm{N}-\mathrm{H}$ lactam and benzophenone. The mass spectrum for the $\mathrm{N}-\mathrm{H}$ lactam is provided. 


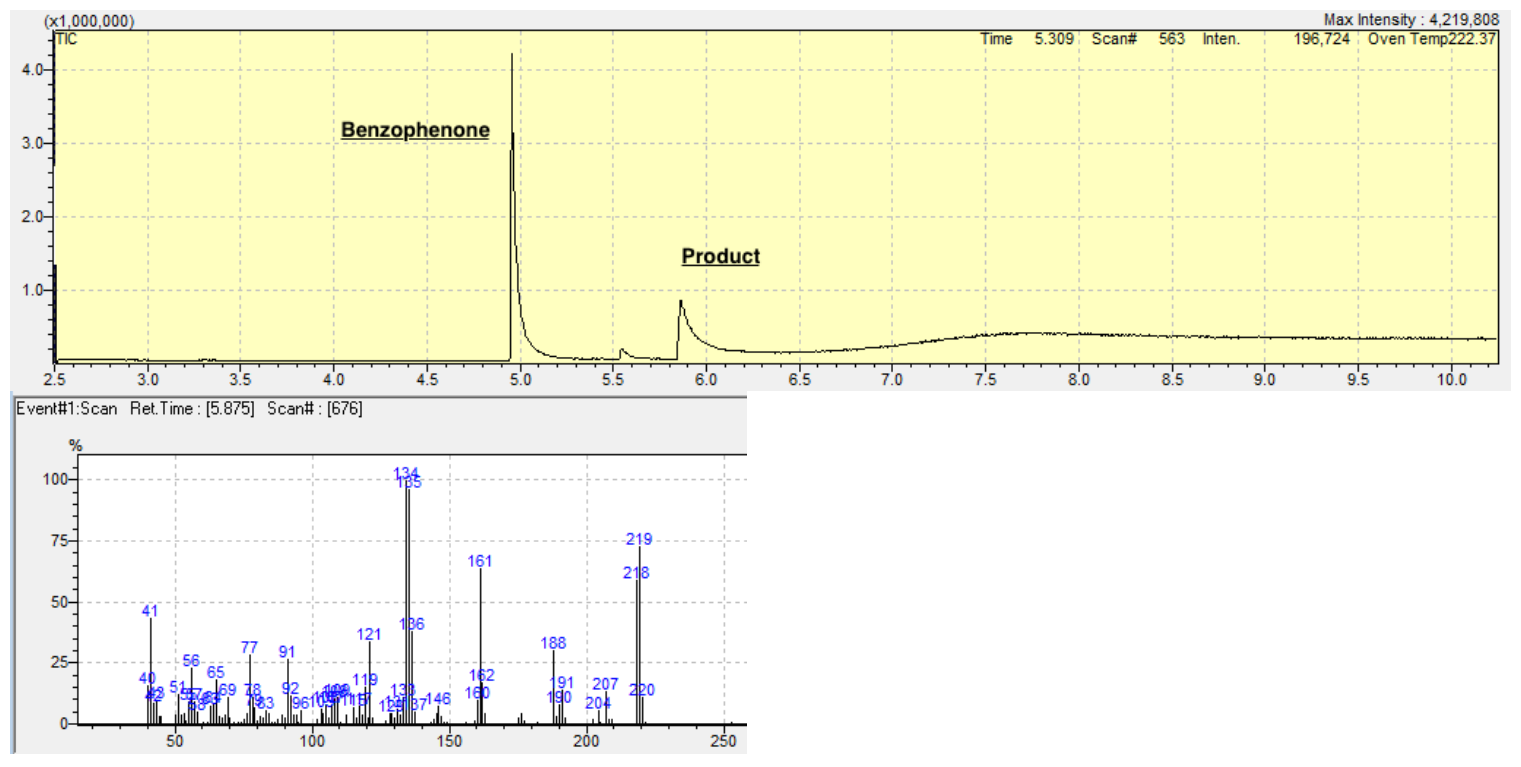




\section{H. Determination of Spirocycle Diastereoisomer}

A full discussion on the determination of the relative stereochemistry for the spirocyclic iminolactone product (2aj) is provided below. For a quick summary outlining key data, please see Figure $\mathbf{S 6}$ on page S56.

For 2aj, COSY and NOESY spectra enabled a clear determination of the relative stereochemistry of the major diastereomer. The ${ }^{1} \mathrm{H}$ NMR spectrum is shown below with the relevant signals labeled (Figure S1):

和

ninisininisin

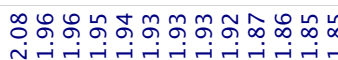

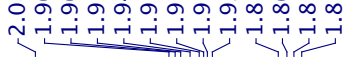

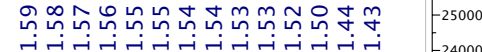

ill

s)
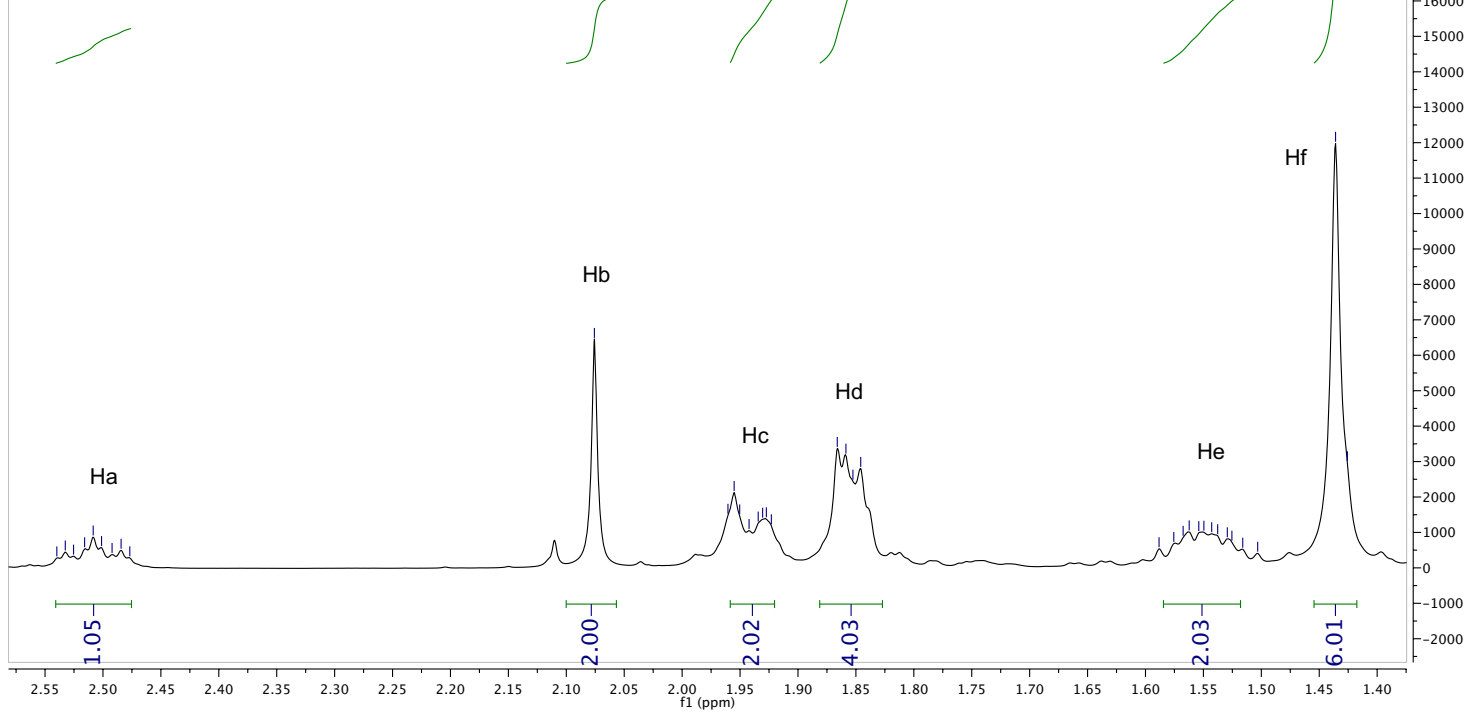

Figure S1. ${ }^{1} \mathrm{H}$ NMR spectrum of $\mathbf{2 a j}$.

The signal for $\mathbf{H}_{\mathbf{a}}$ is a triple triplet with coupling constants of 12.2 and $3.70 \mathrm{~Hz}$. This is indicative of a proton that is coupled to identical axial and equatorial protons. Based on this coupling pattern, the chemical shift, and the integration of the signal, $\mathbf{H}_{\mathbf{a}}$ can be assigned as the benzylic proton.

Investigation of the COSY spectrum (Figure S2) indicates $\mathbf{H}_{\mathbf{a}}$ is coupled to $\mathbf{H}_{\mathrm{c}}$ and $\mathbf{H}_{\mathbf{e}}$. Therefore, $\mathbf{H}_{\mathbf{c}}$ and $\mathbf{H}_{\mathrm{e}}$ can be assigned to the equatorial and axial protons on the carbon adjacent to that bearing $\mathbf{H}_{\mathbf{a}} \cdot \mathbf{H}_{\mathbf{e}}$ is assigned as the axial protons adjacent to $\mathbf{H}_{\mathbf{a}}$, given that the cross-peak for that coupling is large. $\mathbf{H}_{\mathbf{c}}$ is therefore the equatorial protons adjacent to $\mathbf{H}_{\mathbf{a}}$. Importantly, $\mathbf{H}_{\mathrm{e}}$ also couples strongly with $\mathbf{H}_{\mathrm{c}}$.

Consistent with these assignments, $\mathbf{H}_{\mathrm{e}}$ and $\mathbf{H}_{\mathrm{c}}$ also couple with $\mathbf{H}_{\mathrm{d}}$, which must be the 4 protons in the two remaining methylene units. Importantly, $\mathbf{H}_{\mathbf{d}}$ does not couple with $\mathbf{H}_{\mathbf{a}}$, which supports that $\mathbf{H}_{\mathbf{d}}$ must be the methylene protons distal to $\mathbf{H}_{\mathbf{a}}$ and that $\mathbf{H}_{\mathrm{e}}$ and $\mathbf{H}_{\mathrm{c}}$ are indeed the methylene units proximal to $\mathbf{H}_{\mathbf{a}}$. 


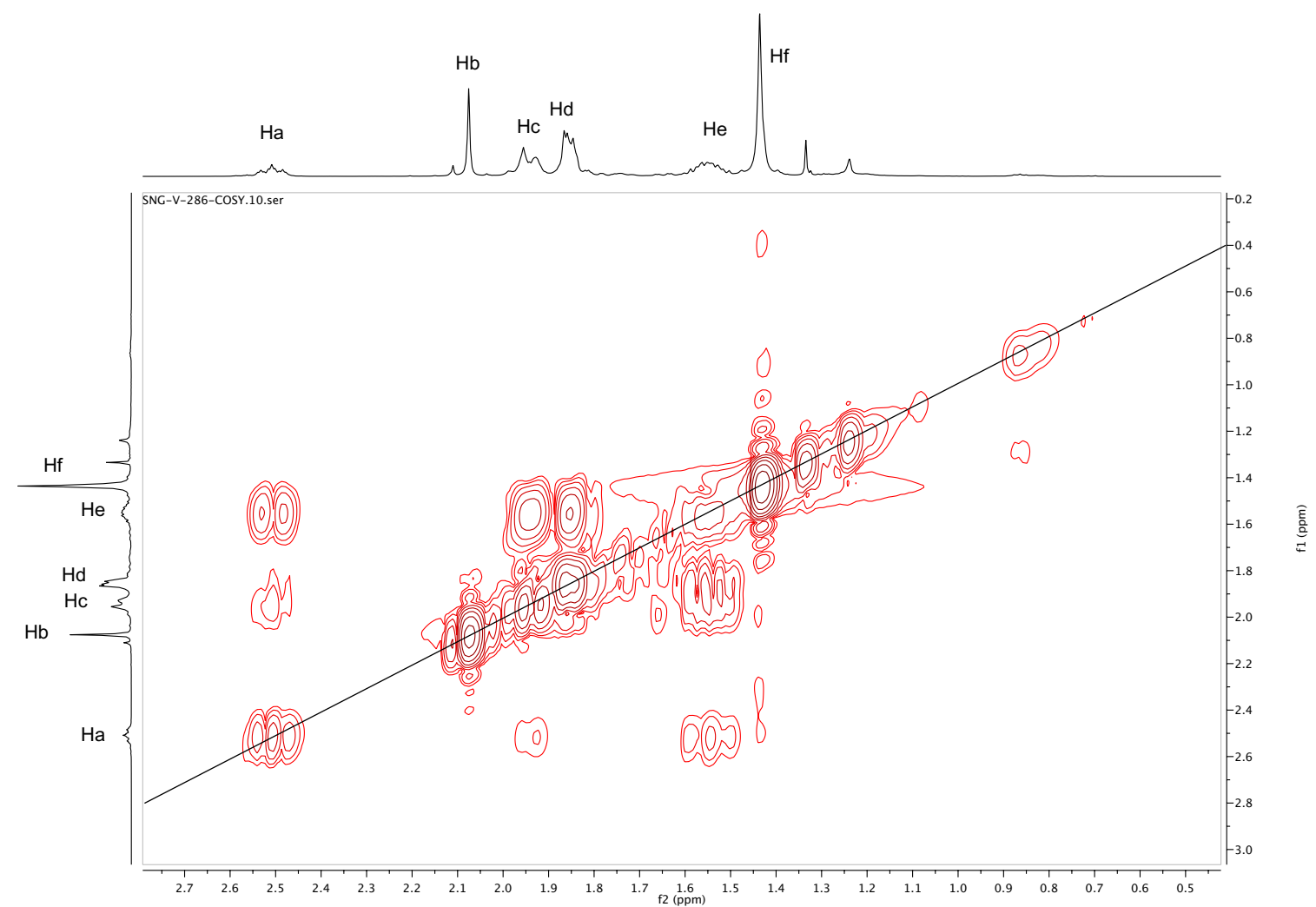

Figure S2. COSY spectrum of 2aj.

Finally, $\mathbf{H}_{\mathrm{b}}$ and $\mathbf{H}_{\mathrm{f}}$ do not display any coupling and are assigned as the protons in the iminolactone methylene and methyl units, respectively. Based on these analyses, the protons in the product structure can be assigned as shown below (Figure S3). A summary of the couplings is also provided.

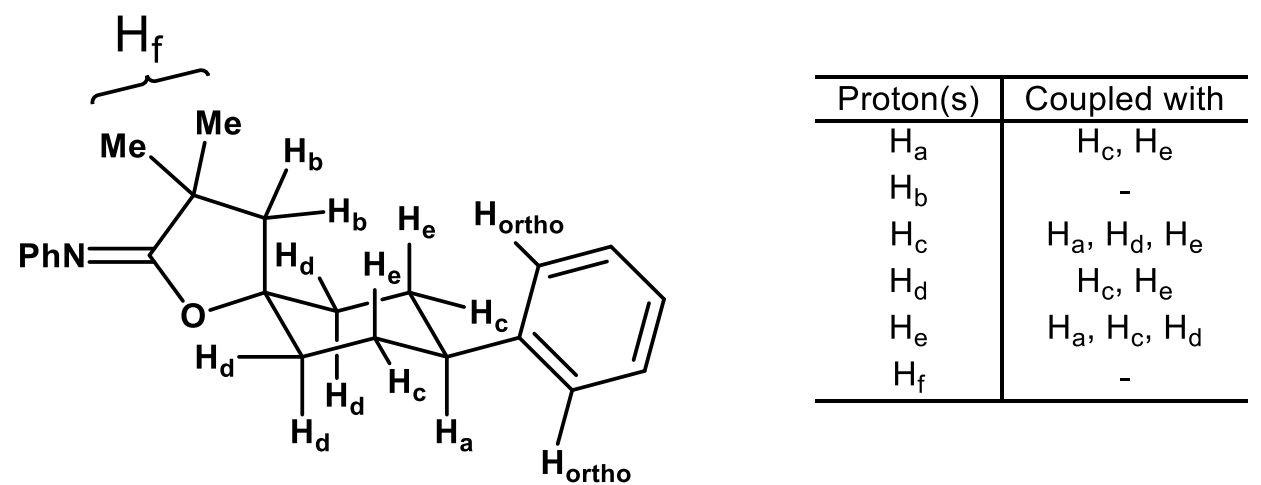

Figure S3. Summary of coupling in 2aj.

With the protons identified, the NOESY spectrum can be analyzed to provide insight into the relative stereochemistry (Figure $\mathbf{S 4})$. The methyl groups $\left(\mathbf{H}_{\mathrm{f}}\right)$ show an NOE with the iminolactone methylene unit $\left(\mathbf{H}_{b}\right)$. Following this network, $\mathbf{H}_{b}$ shows a strong NOE with $\mathbf{H}_{e}$. This NOE network is sufficient to place $\mathbf{H}_{\mathrm{e}}$ and $\mathbf{H}_{\mathrm{b}}$ on the same side of the molecule and to therefore assign the stereochemistry as trans. To solidify this assignment, we note that $\mathbf{H}_{\mathrm{e}}$ shows an NOE with aryl protons, likely the ortho protons on the trans-phenyl unit. Importantly, $\mathbf{H}_{\mathbf{a}}$ shows a clear NOE with the same aryl protons. This particular arrangement is only possible with the structure proposed abov e. The NOE correlations are summarized below (Figure S5). 


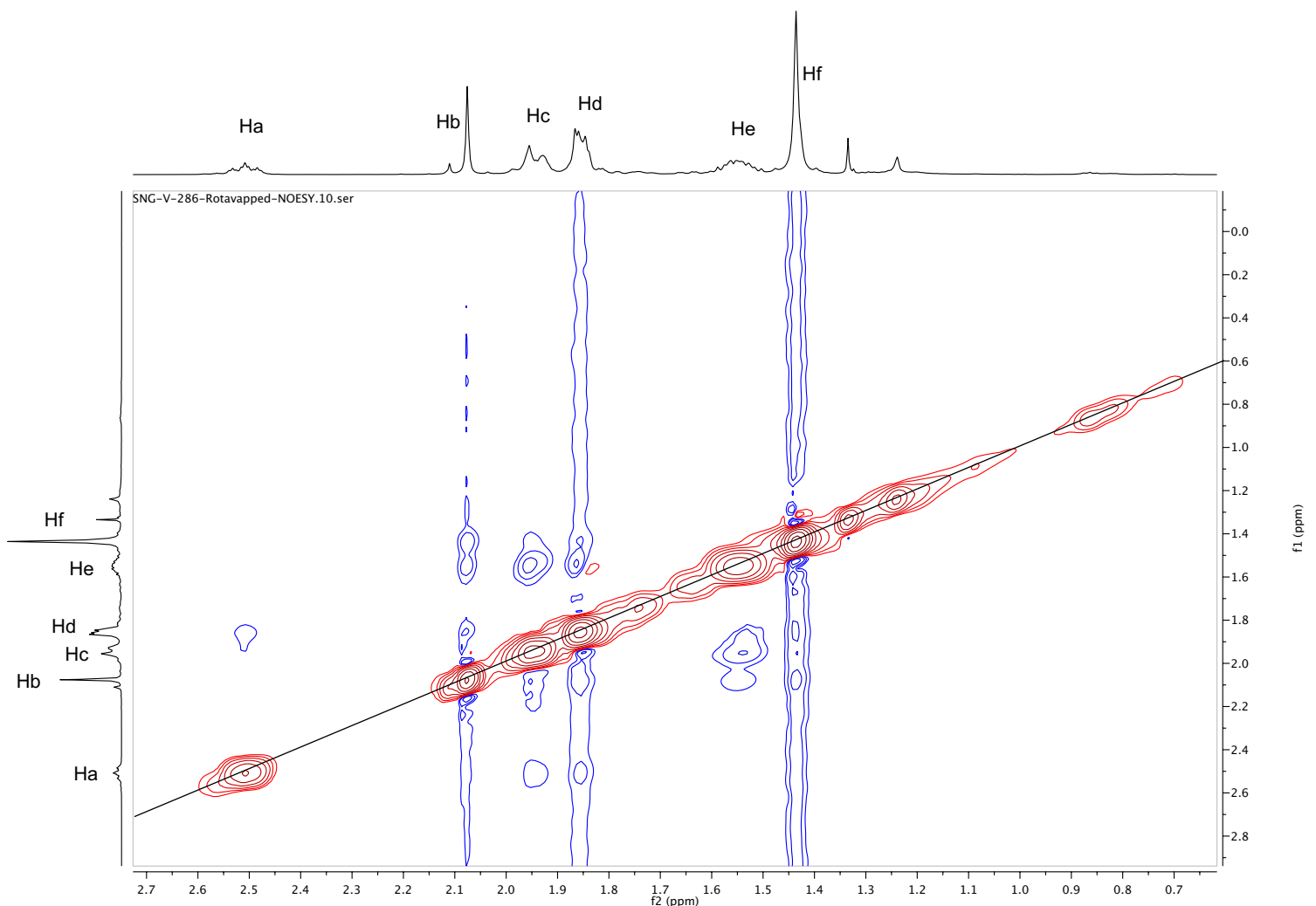

Figure S4. NOESY spectrum of 2aj.

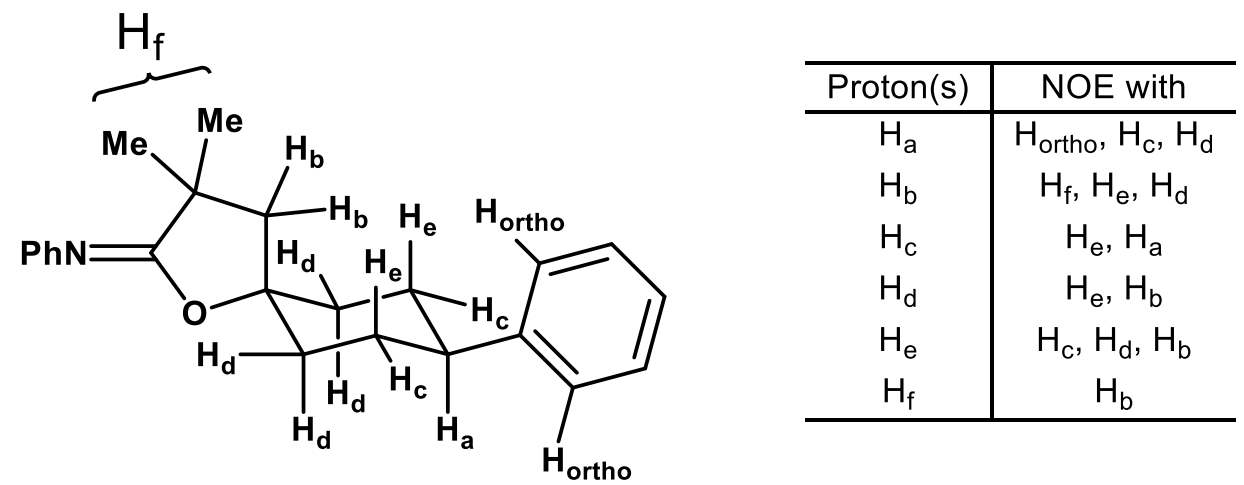

Figure S5. Summary of NOESY correlations in 2aj.

A summary of this entire analysis is shown below with the key findings that enable the determination of the relative stereochemistry (Figure S6). 


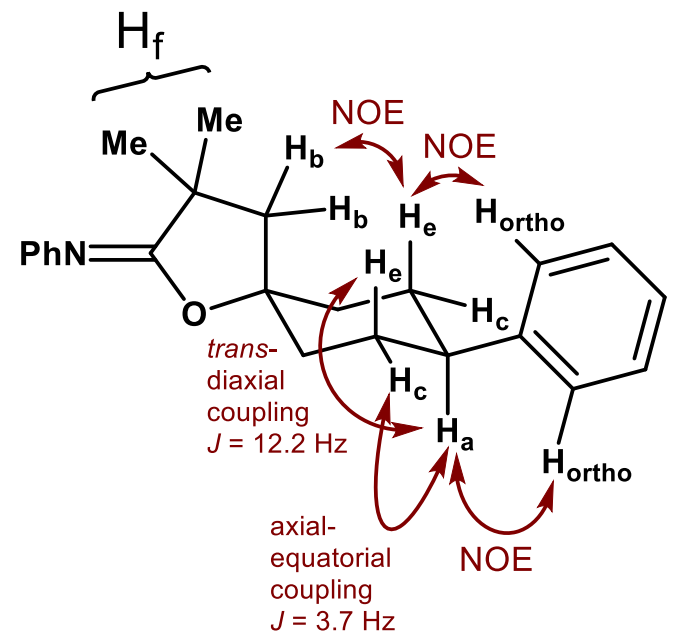

Figure S6. Key spectral data for assignment of relative stereochemistry for $\mathbf{2} \mathbf{a j}$. 


\section{Additional Mechanistic Information and Catalytic Cycle}

This information is intended to supplement the mechanistic experimentation detailed in the manuscript.

\section{Probing the Intermediacy of the Oxocarbenium}

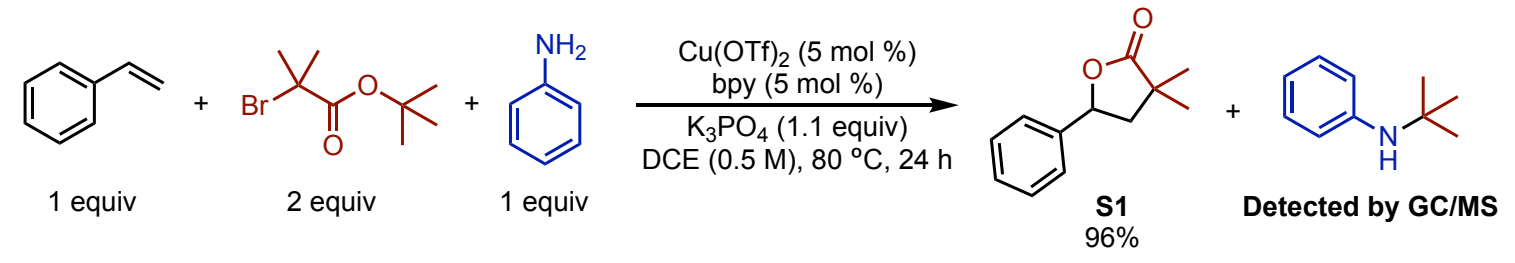

Figure S7. S1 formation from tert-butyl 2-bromoisobutyrate.

When tert-butyl 2-bromoisobutyrate, styrene, and aniline are subjected to the reaction conditions, the usual carboamination product (the corresponding iminolactone) is not obtained. Rather, nearly all the styrene is converted to the above $\gamma$-lactone (S1, Figure S7). Additionally, $N$-tert-butylaniline is observed by GC/MS analysis of the crude reaction mixture. We attribute the formation of these products to the intermediacy of the oxocarbenium species, which can either undergo E2 elimination or nucleophilic substitution to produce the observed lactone and alkylated nucleophile (Figure S8). Since the tertbutylaniline is detected only in minute quantities, we conclude that elimination is the predominant pathway of $\mathbf{S} 1$ formation.

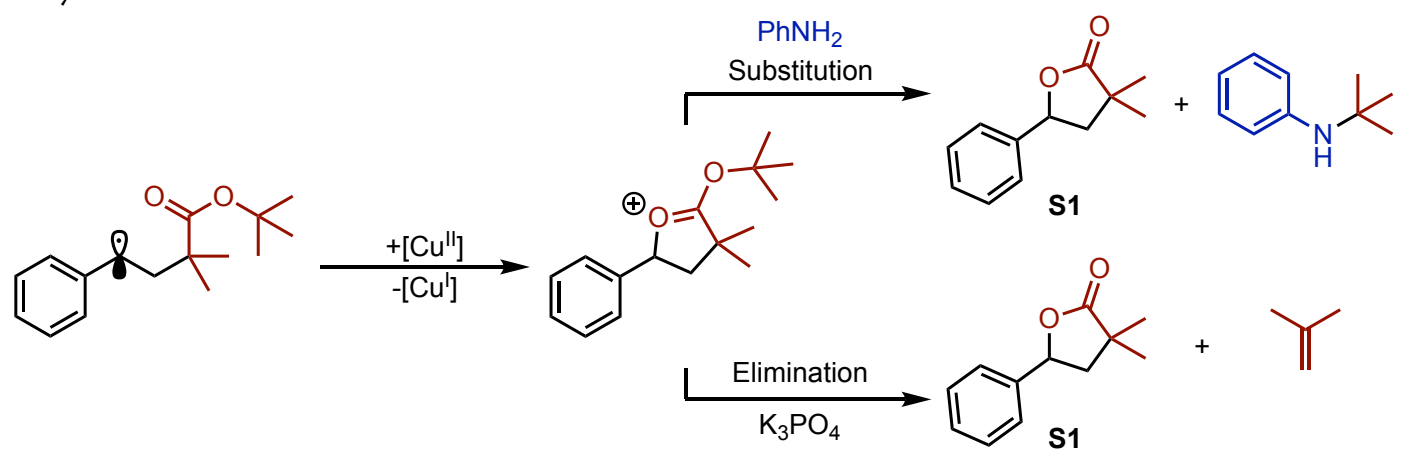

Figure S8. Proposed mechanism of lactone and $\mathrm{N}$-tert-butylaniline formation.

When the reaction is conducted in the absence of an amine nucleophile, the oxocarbenium would still be expected to form. However, without a nucleophile present, alternative decomposition pathways would be anticipated to occur to form other observable products. Indeed, it was observed that in the absence of the amine, the Heck-type product $\mathbf{S 2}$ is formed, albeit in very low yield. However, when the reaction is conducted with $5 \mathrm{~mol} \%$ added aniline or $\mathrm{N}$-methylaniline, $\mathbf{S 1}$ is formed in very high yield. This result suggests that the amine nucleophile is itself incorporated into the catalyst and influencing activity. Our current hypothesis is that $\mathbf{S 1}$ is formed through elimination to form ethylene and $\mathbf{S 2}$ is formed through elimination from the homobenzylic site.

These results are still consistent with the formation of oxocarbenium intermediates. In such intermediates, resonance stabilization of the ketyl-type carbon will distribute the positive charge between each oxygen group. In the case of the tert-butyl ester, the oxygen bearing the tert-butyl group takes on more positive charge, as the tert-butyl group can inductively stabilize the neighboring cationic oxygen. In this case, elimination from the tert-butyl group affords predominantly the lactone product. In the case of the ethyl ester in the absence of amine nucleophiles, the oxygen at the benzylic position likely takes on more of the positive charge, as the neighboring benzylic carbon can better stabilize the cationic oxygen. 
In this case, elimination occurs from the homobenzylic site to form the observed Heck-type product. The results of these studies are outlined below (Figure S9).

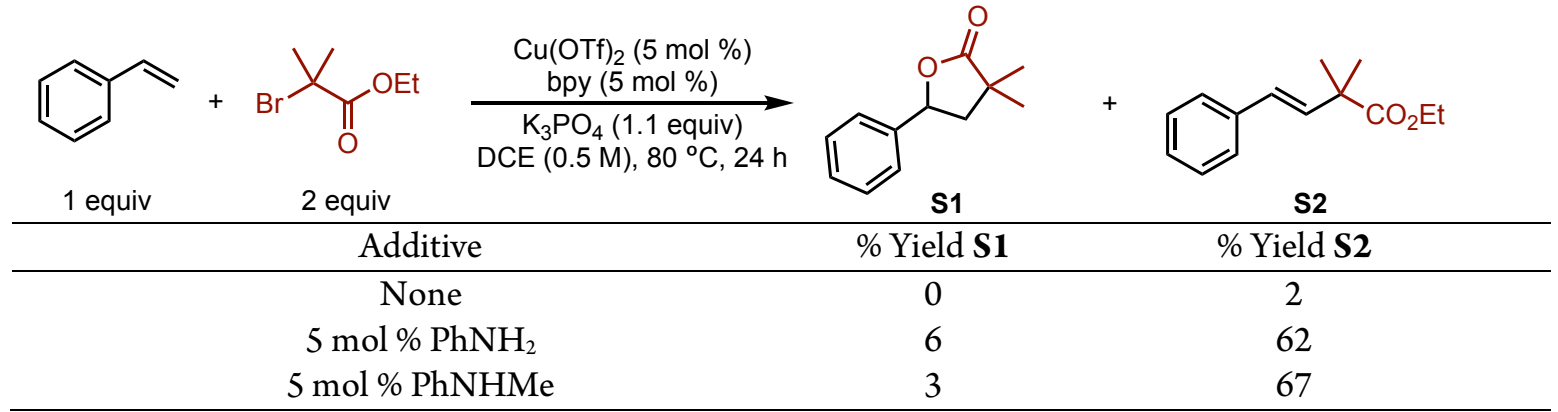

Figure S9. Reactions conducted in the absence of a stoichiometric nucleophile.
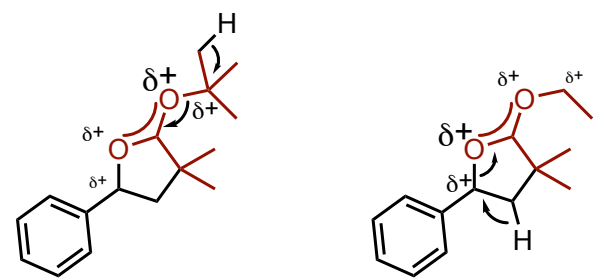

Figure S10. Distribution of charge in oxocarbenium intermediates.

A catalytic cycle for this transformation is proposed below. The reaction initiates with activation of the alkyl halide substrate to produce a carbon-centered radical. This radical then combines with the olefin in an addition reaction to form a radical addition intermediate. Subsequent oxidation affords the key oxocarbenium ion intermediate and closes the catalytic cycle. Quenching of the oxocarbenium with the amine nucleophile thus produces the product.

Plausible Catalytic Cycle

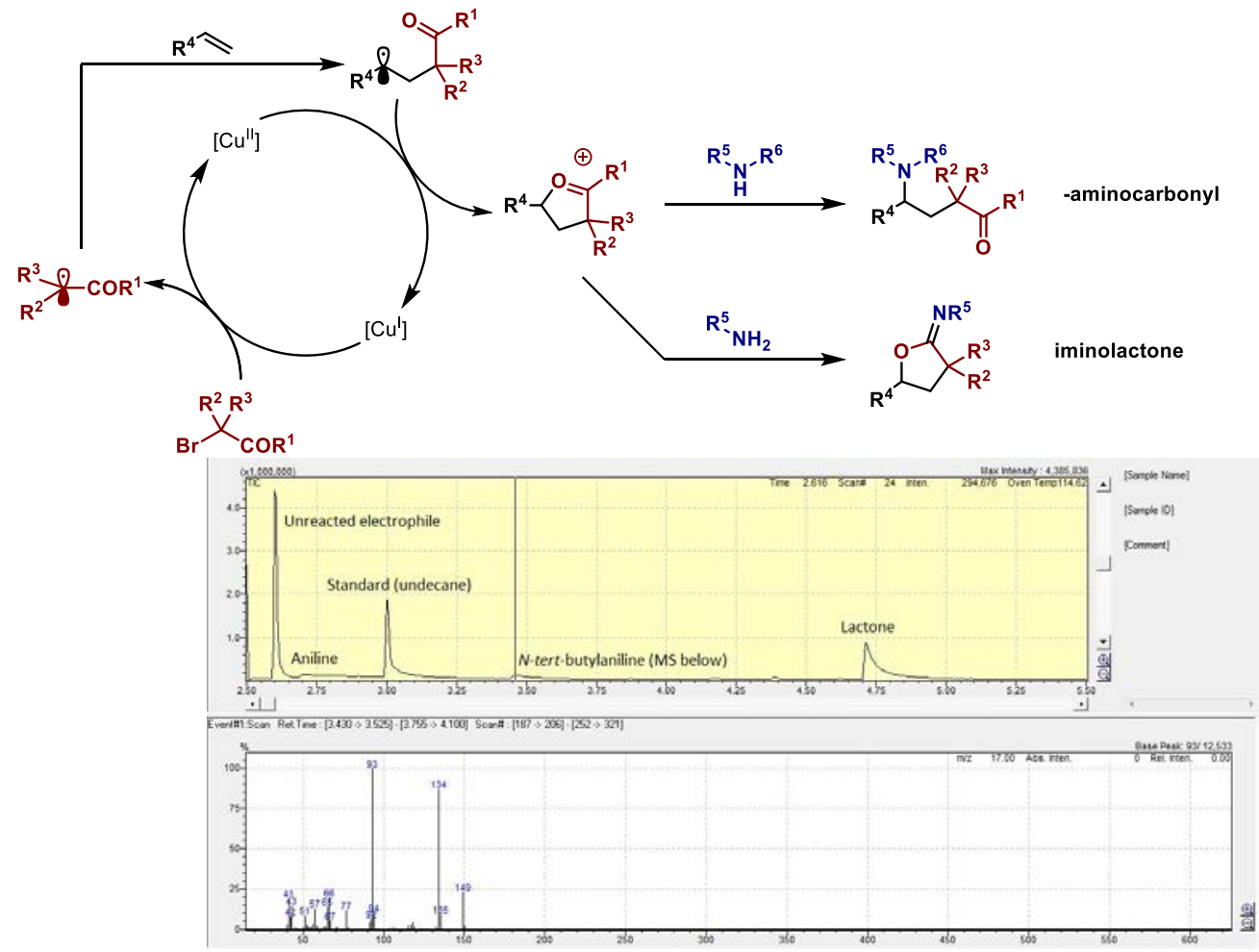



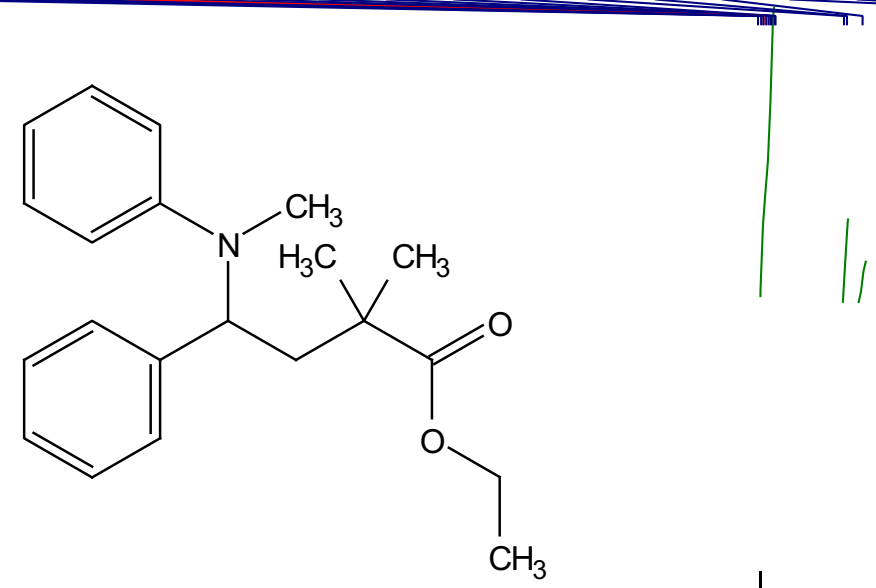

$2 \mathbf{a}$

${ }^{1} \mathrm{H} N M R, 500 \mathrm{MHz}, \mathrm{CDCl}_{3}$
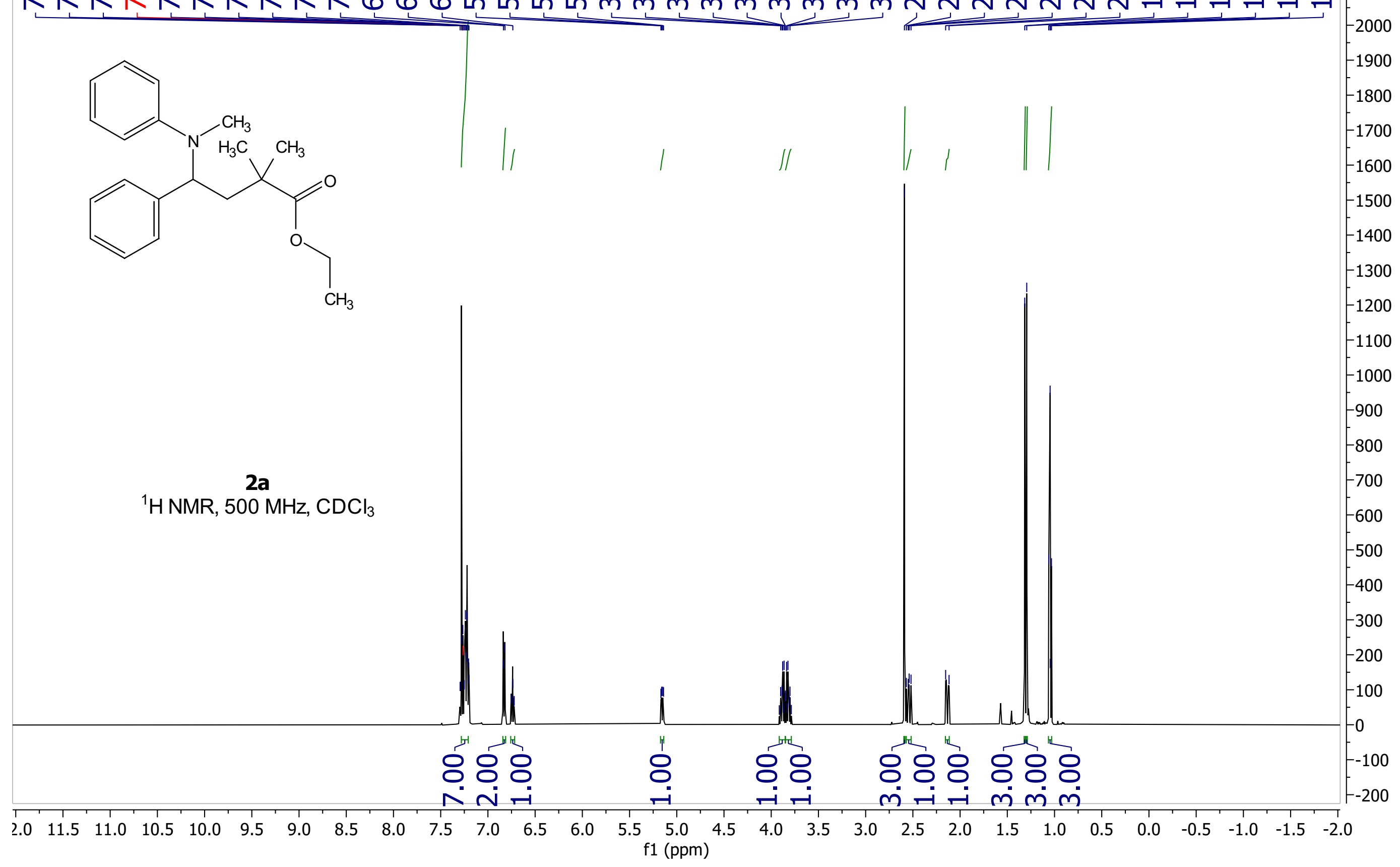


\section{$m$}
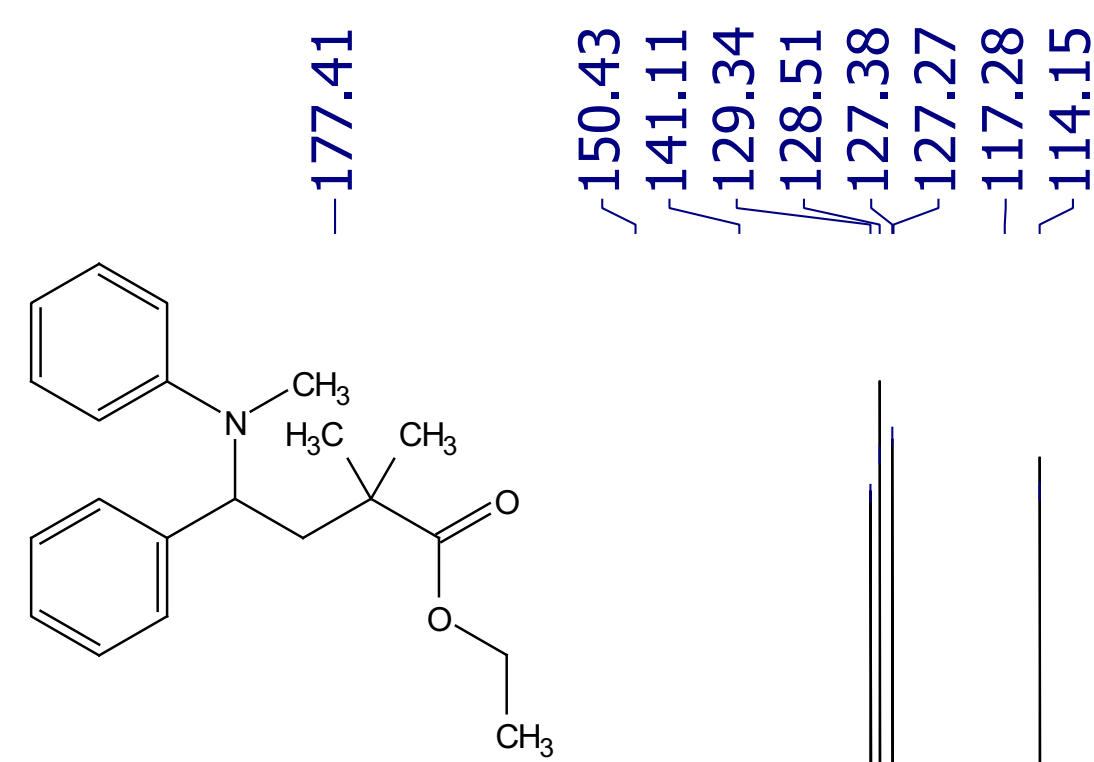

$2 a$

${ }^{13} \mathrm{C} \mathrm{NMR}, 126 \mathrm{MHz}, \mathrm{CDCl}_{3}$

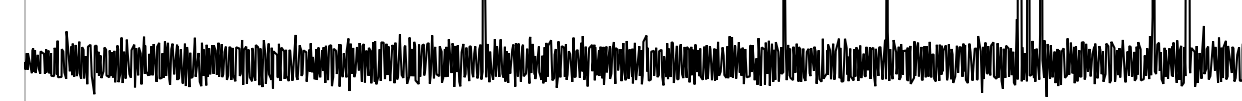

$\infty 6$

$\infty \infty$

ب
乐

ๆั 


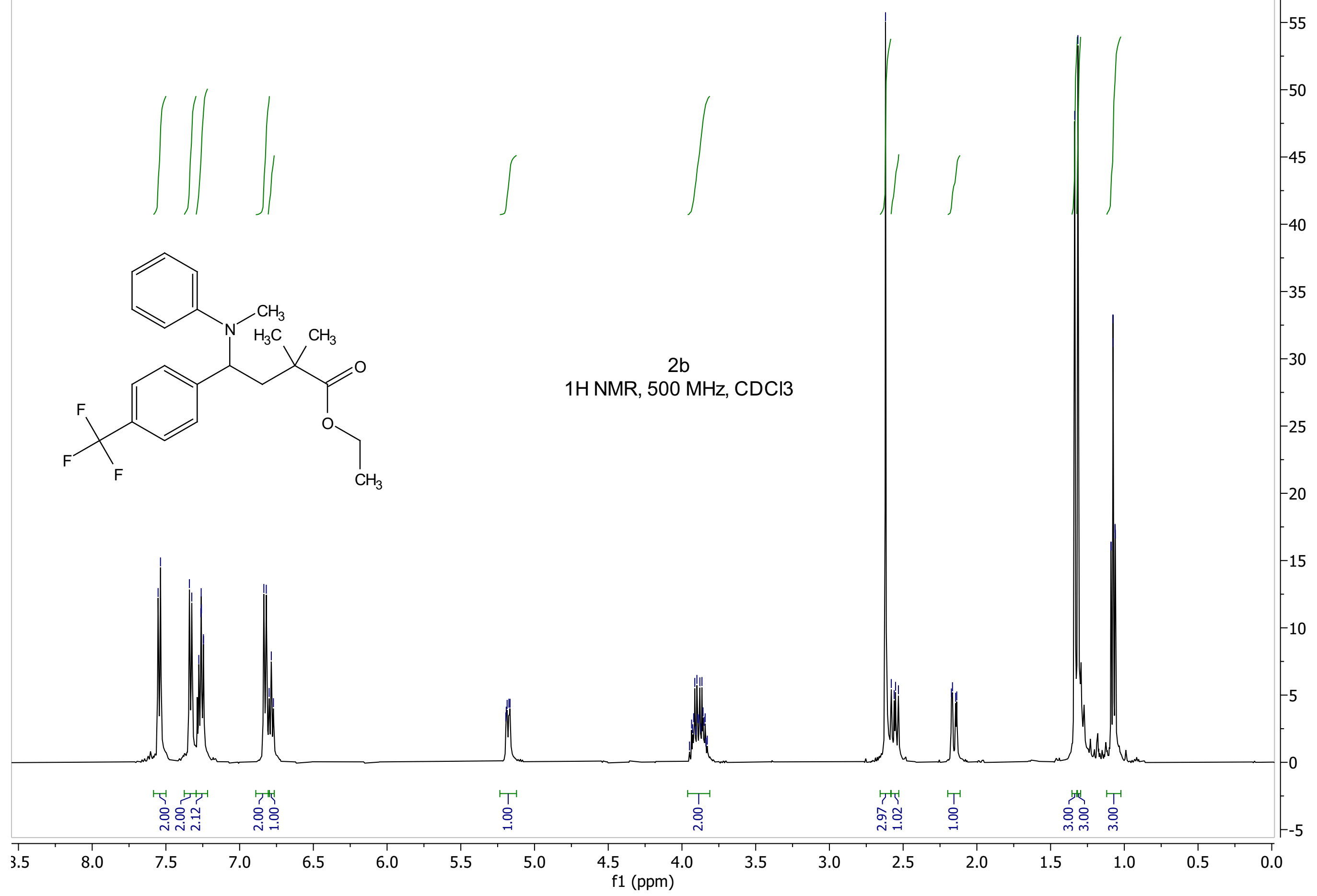


<smiles>C=CCN(C)C(CC(C)(C)C(=O)OCC)c1ccc(C(F)(F)F)cc1</smiles>

13C NMR, $126 \mathrm{MHz}, \mathrm{CDCl} 3$ 
<smiles>CCOC(=O)C(C)(C)CC(c1ccc(C(F)(F)F)cc1)N(C)c1ccccc1</smiles> 


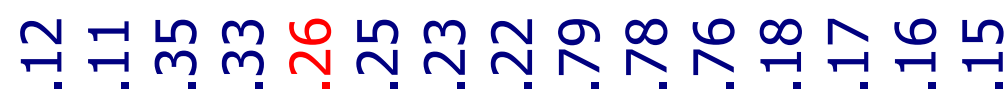
$\infty \underbrace{\infty} N$ N N

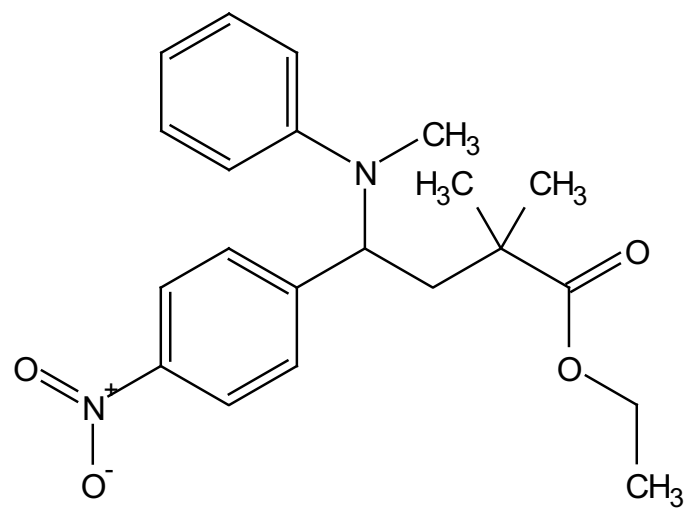

$2 c$

${ }^{1} \mathrm{H} \mathrm{NMR}, 500 \mathrm{MHz}, \mathrm{CDCl}_{3}$

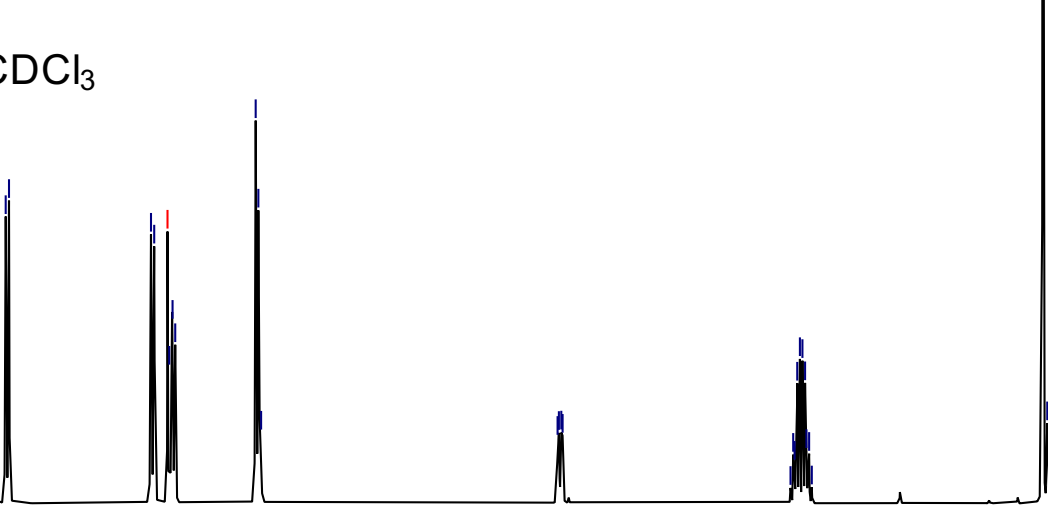
L

の

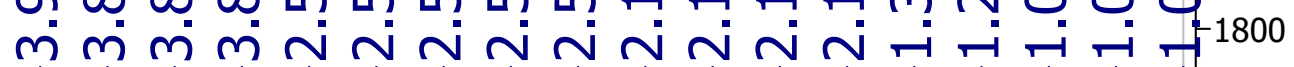
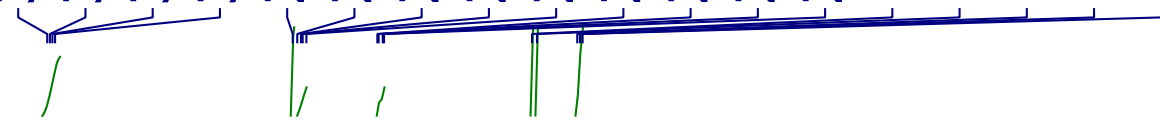

ㄱ 8808

$8.0 \quad 7.5$ 

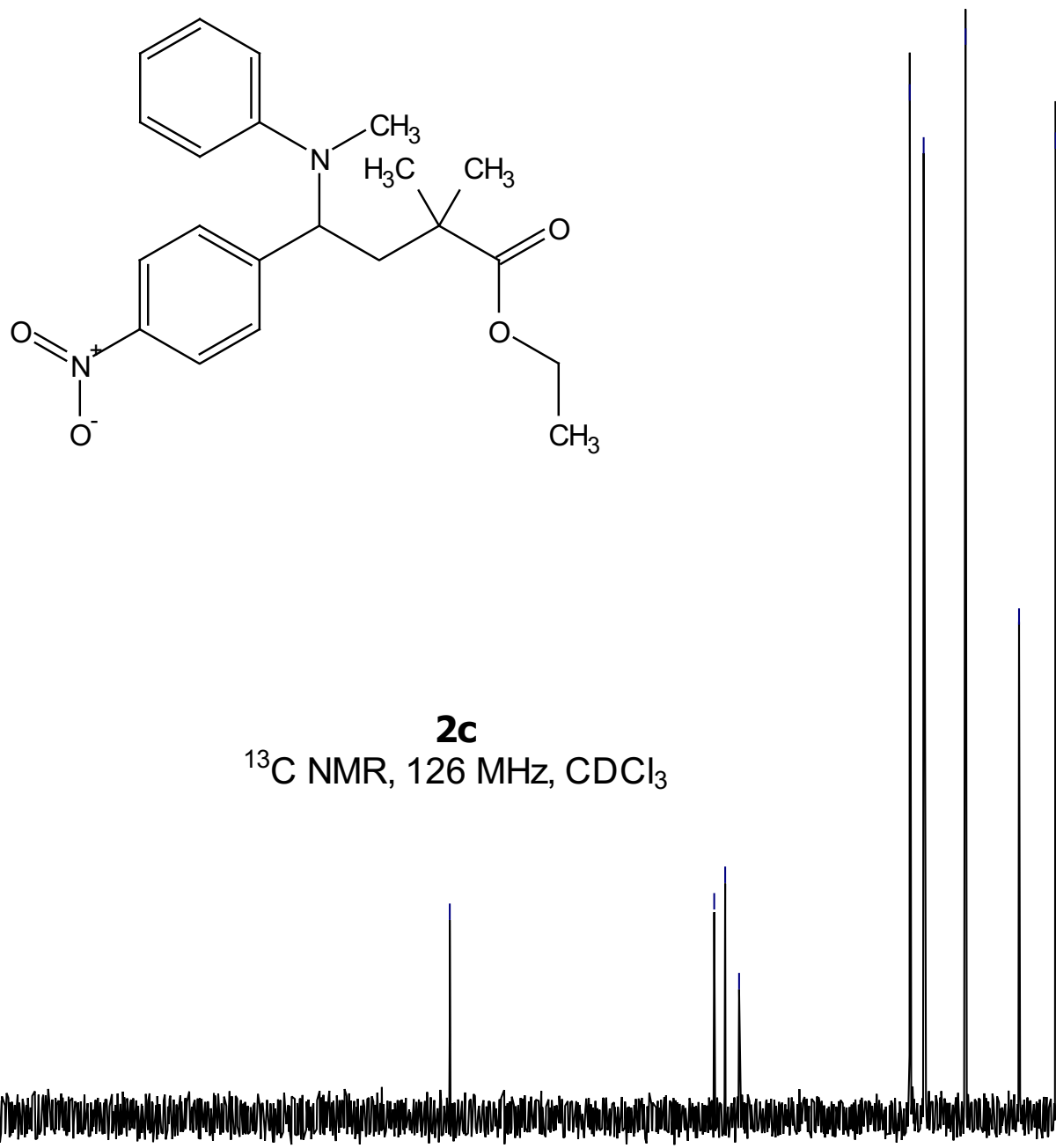

${ }^{13} \mathrm{C}$ NMR, $126 \mathrm{MHz}, \mathrm{CDCl}_{3}$$$
\text { z }
$$

$220 \quad 210 \quad 200$
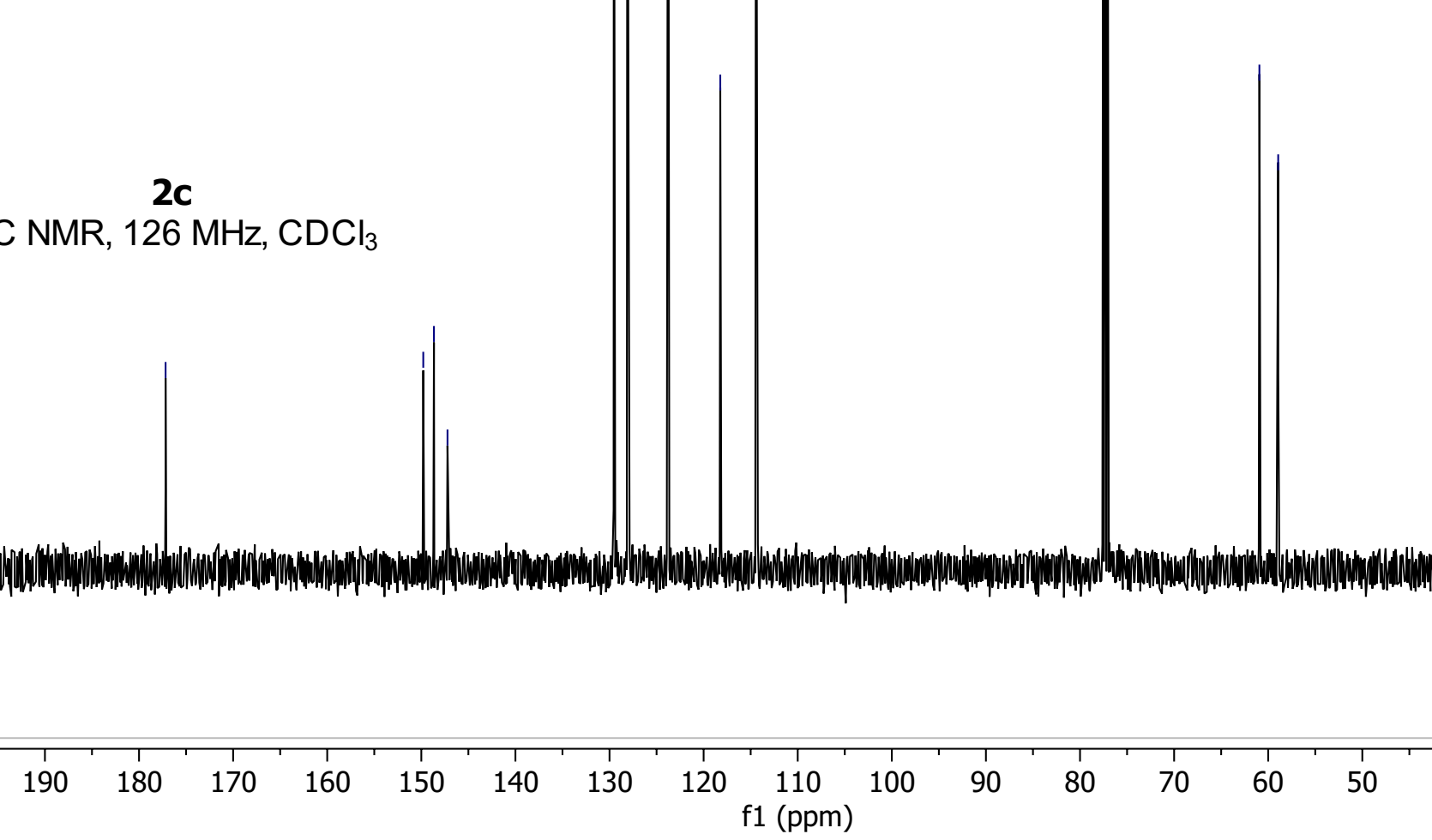

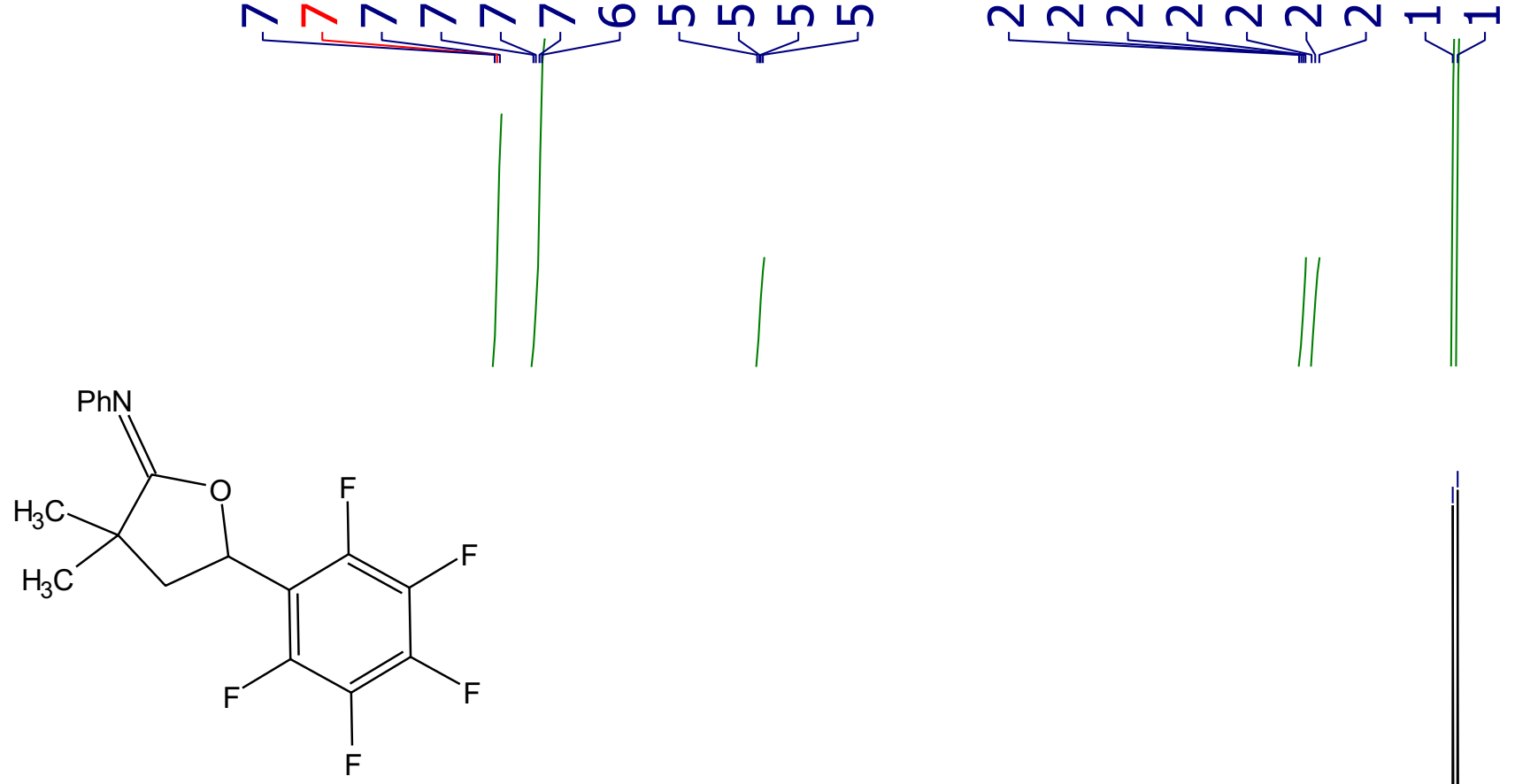

${ }^{1} \mathrm{H}$ NMR, $500 \mathrm{MHz}, \mathrm{CDCl}_{3}$

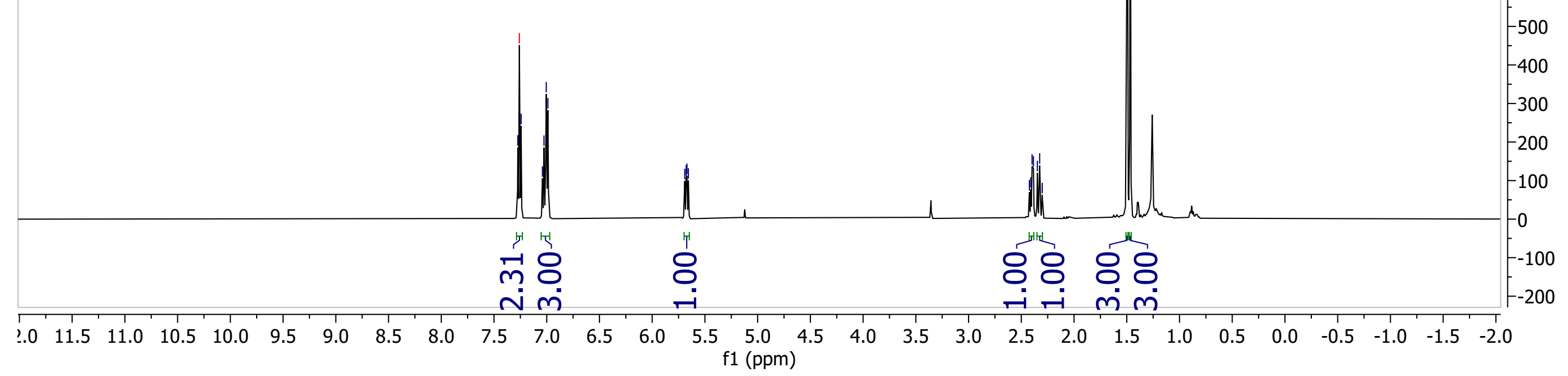




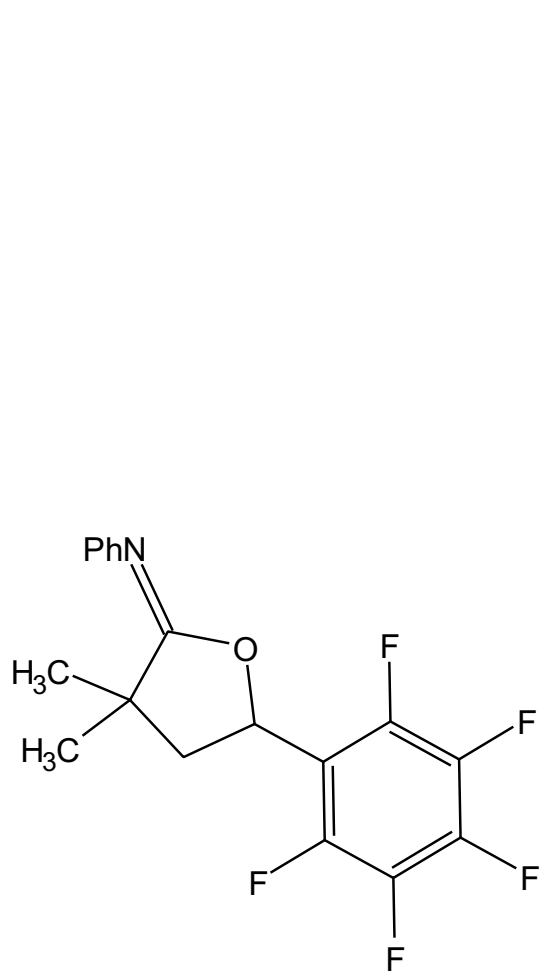

2d

${ }^{19} \mathrm{~F} \mathrm{NMR}, 471 \mathrm{MHz}, \mathrm{CDCl}_{3}$

\section{ơํำ}




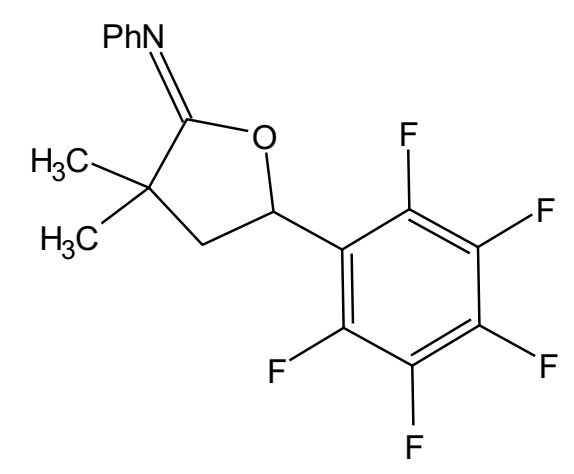

\section{SNG-VI-27-2-13C1.10.fid}

๖๊

m

N. ㄱ. ㄴ. טิ

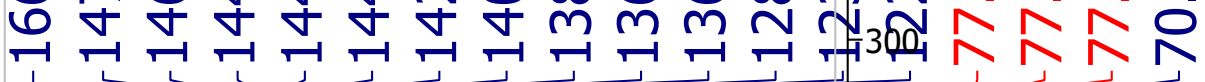

ఠ)

ஓं

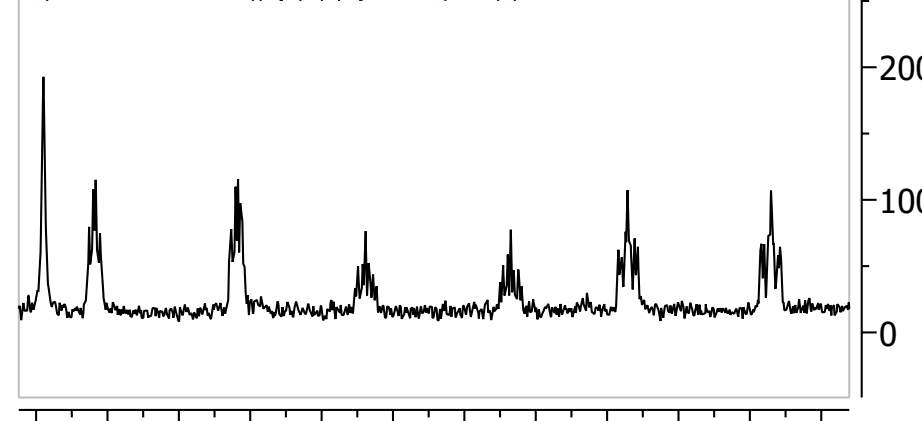

$\begin{array}{llllllllllll}147 & 146 & 145 & 144 & 143 & 142 & 141 & 140 & 139 & 138 & 137 & 136\end{array}$ f1 (ppm)

2d

${ }^{13} \mathrm{C} \mathrm{NMR}, 126 \mathrm{MHz}, \mathrm{CDCl}_{3}$

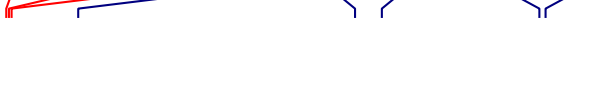



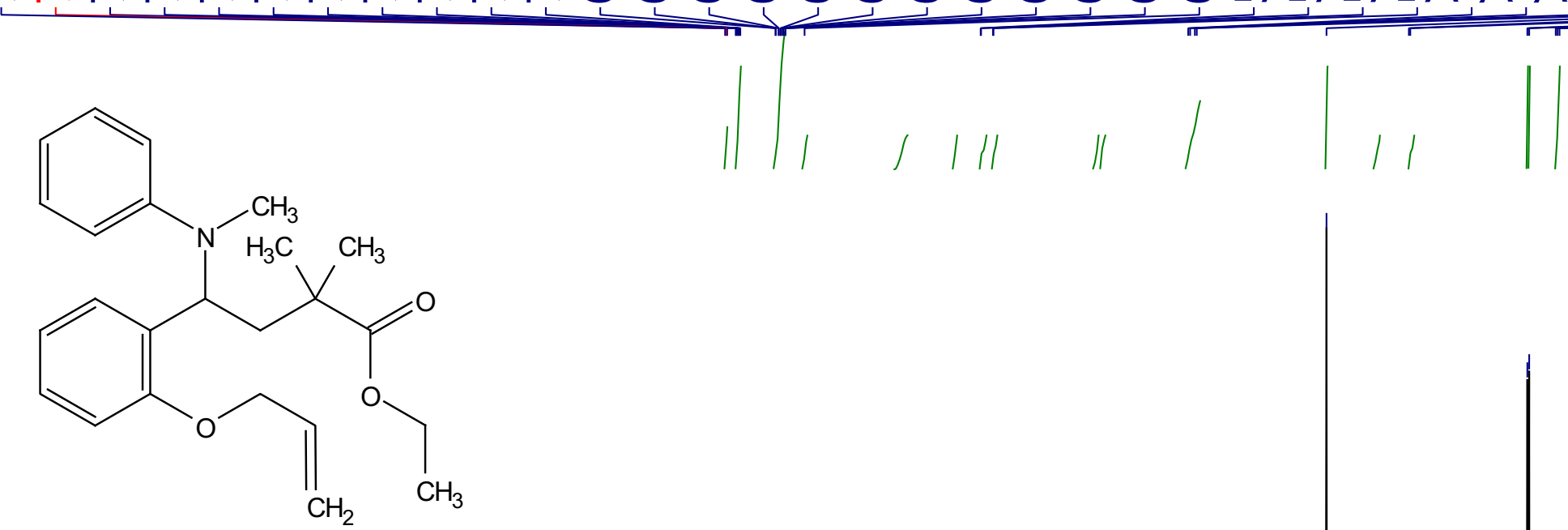

$2 e$

${ }^{1} \mathrm{HNMR}, 500 \mathrm{MHz}, \mathrm{CDCl}_{3}$

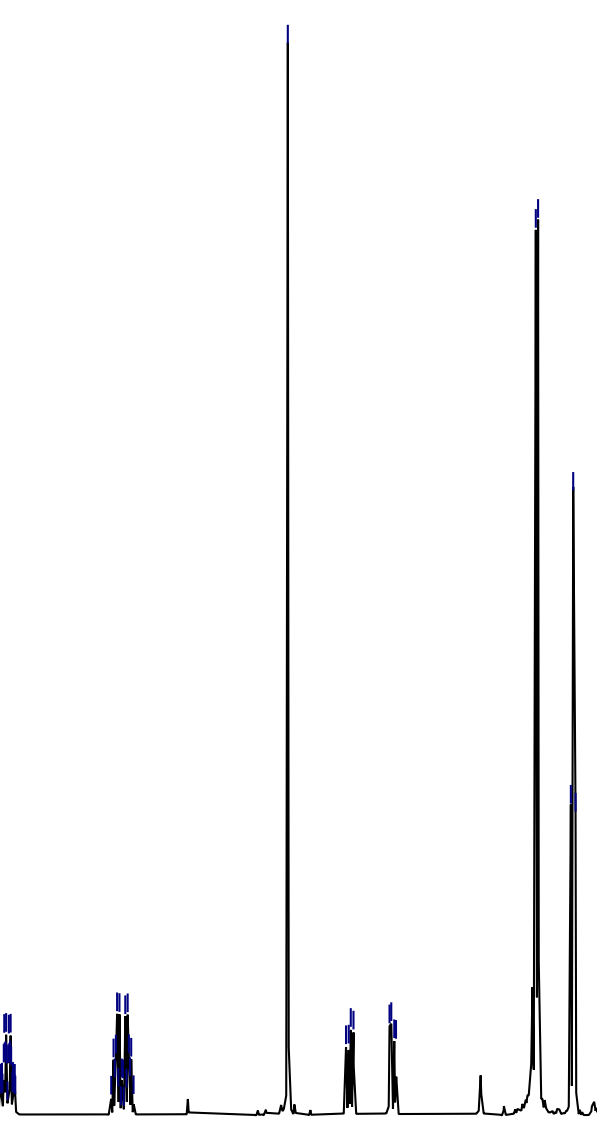




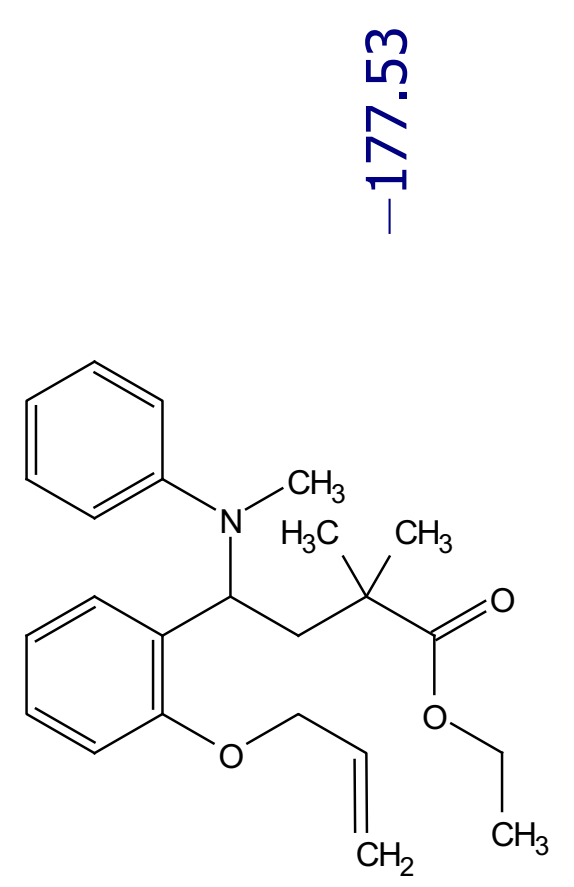

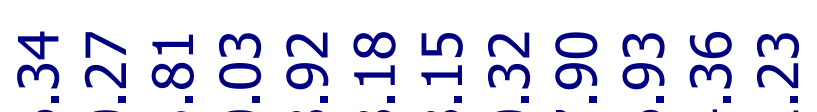
ம்

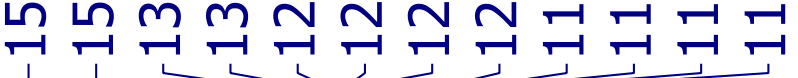

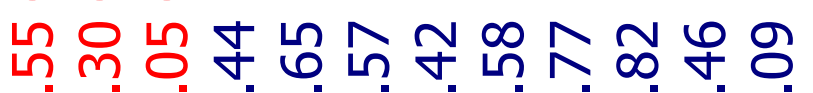

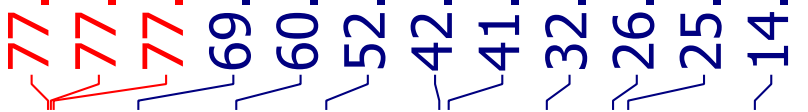

$2 \mathrm{e}$

${ }^{13} \mathrm{C} \mathrm{NMR}, 126 \mathrm{MHz}, \mathrm{CDCl}_{3}$

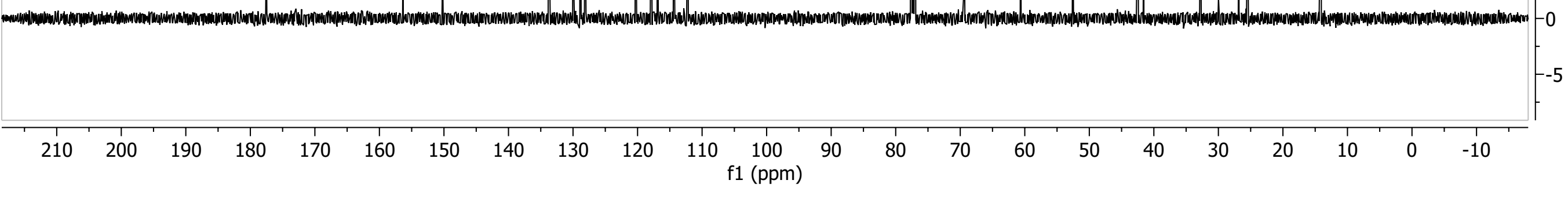



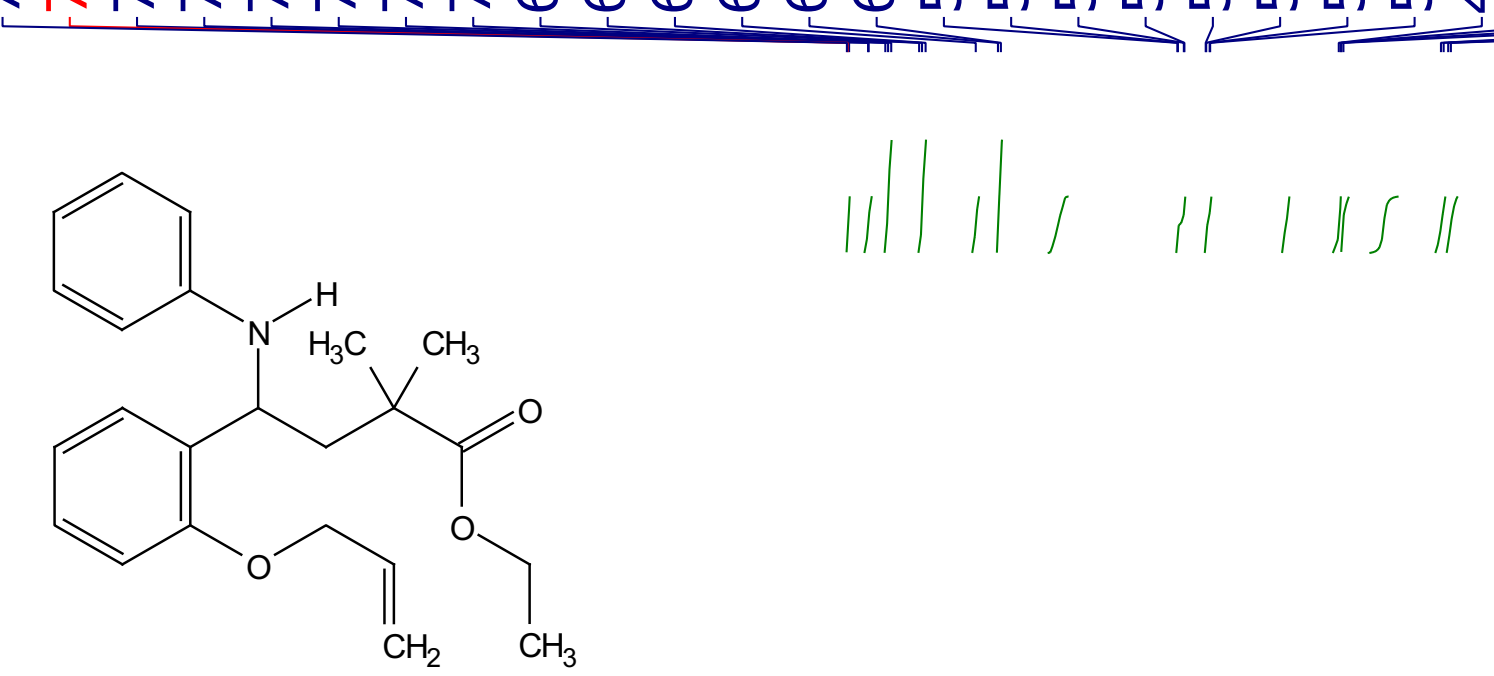

$2 f$

${ }^{1} \mathrm{H} \mathrm{NMR}, 500 \mathrm{MHz}, \mathrm{CDCl}_{3}$

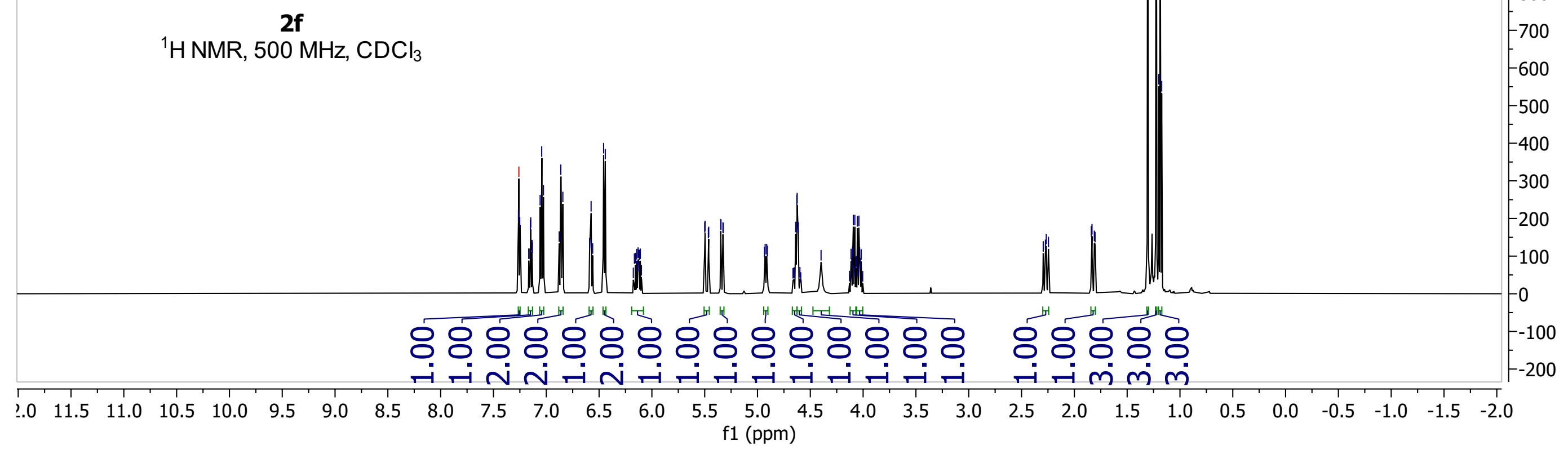




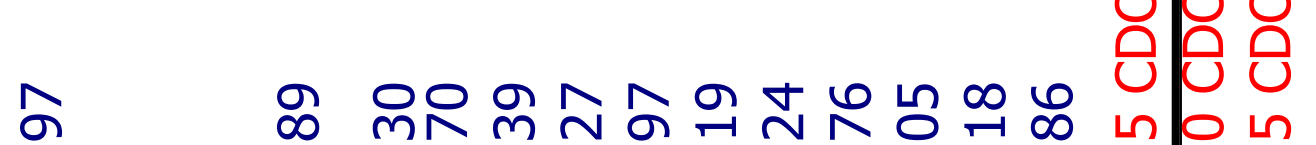

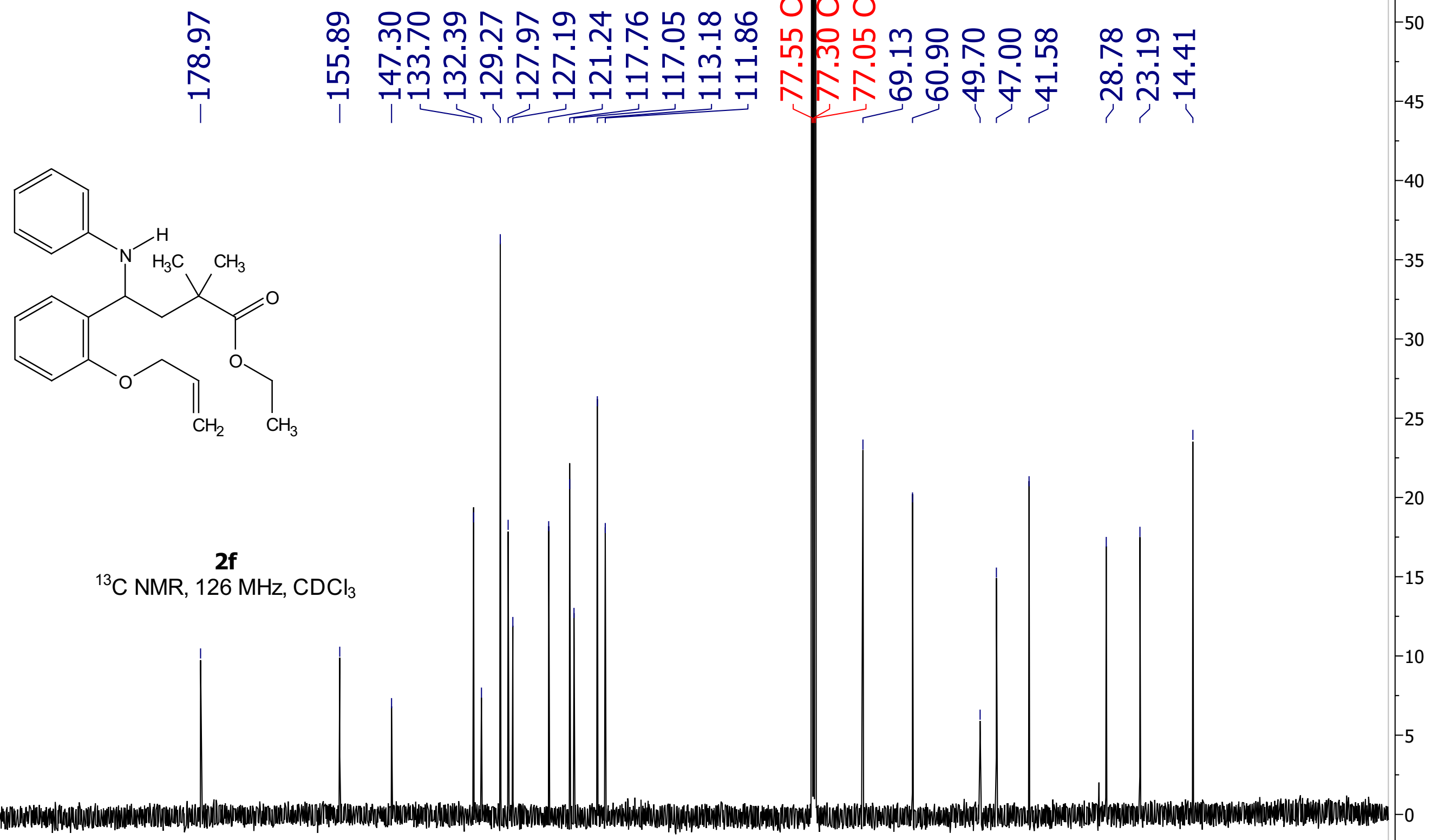

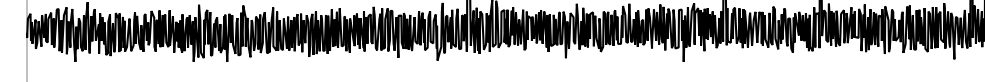

$210 \quad 200$

$190 \quad 180$

170

150

140

120

$110 \quad 100$ f1 (ppm) 
hullthith.

$M \mu \sim \mathrm{L}$

a n

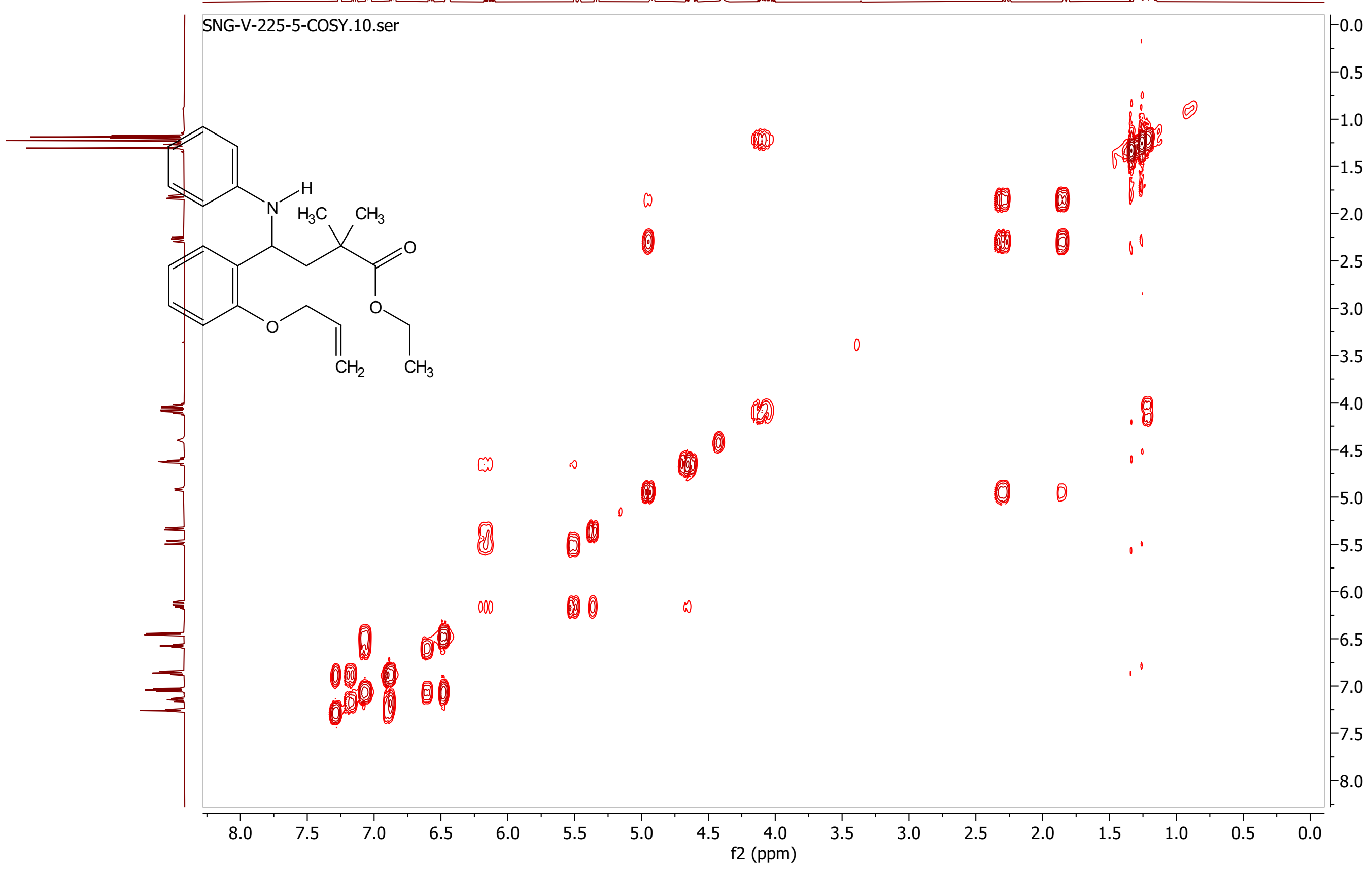

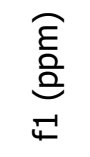




\section{$2 f$}

HSQC spectrum

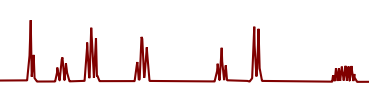

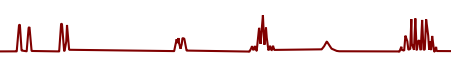

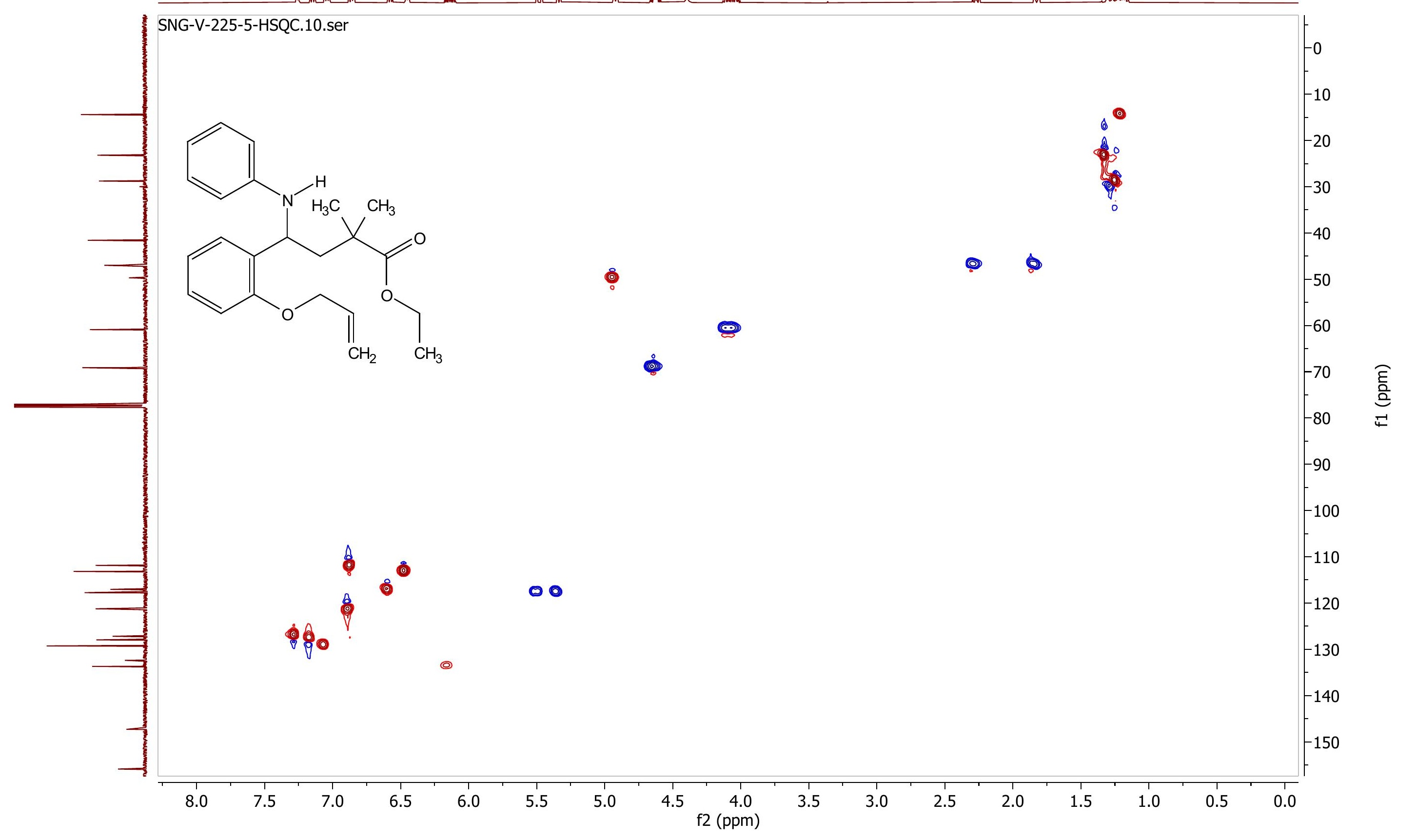




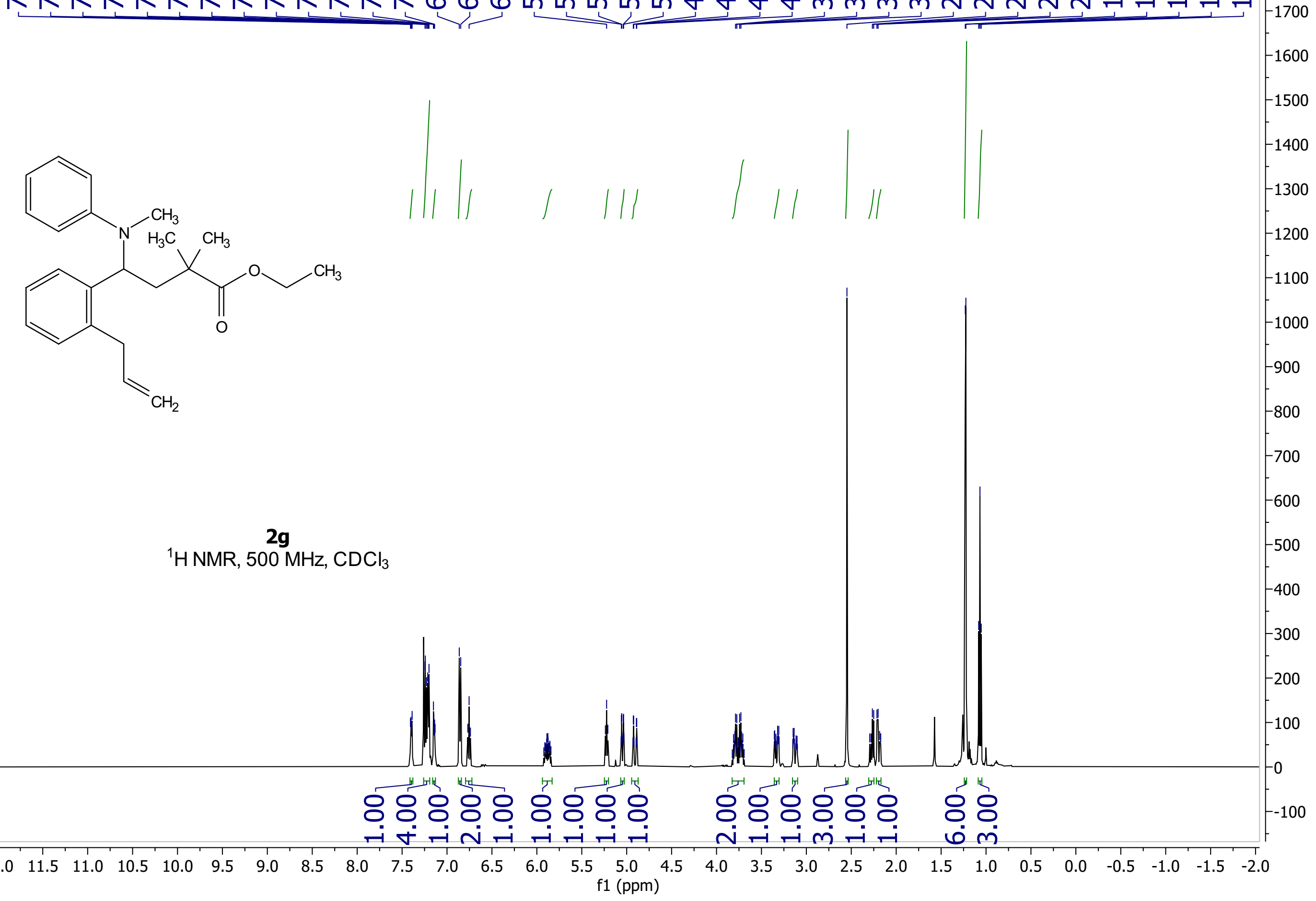


当 ন

N

극
率采

○需已

ㄴํำ

Nㅗ

$2 \mathrm{~g}$

${ }^{13} \mathrm{C} \mathrm{NMR}, 126 \mathrm{MHz}, \mathrm{CDCl}_{3}$

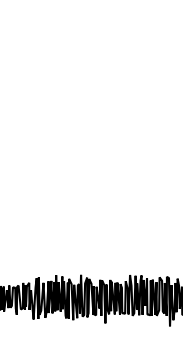



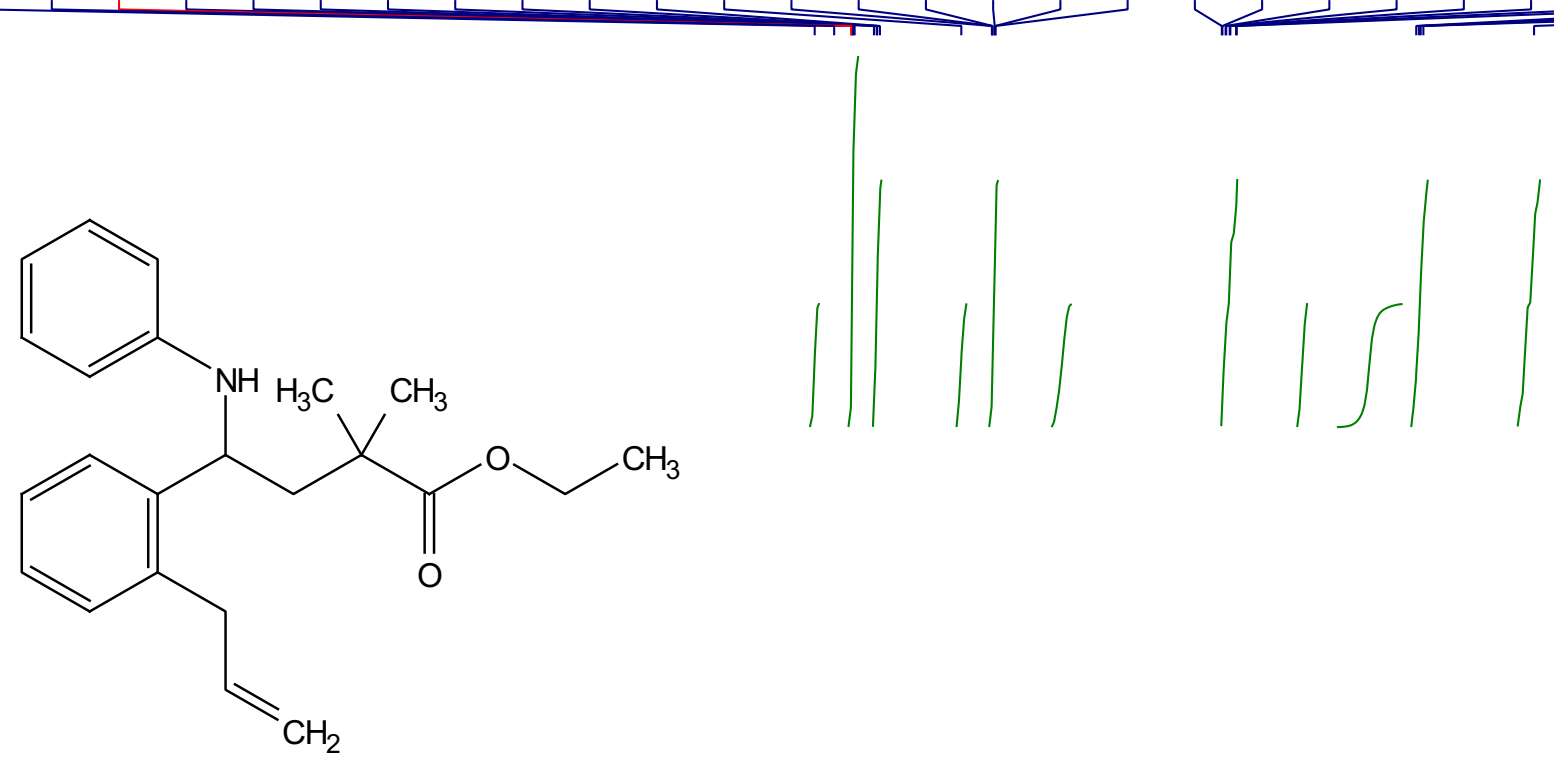

$2 \mathbf{h}$

${ }^{1} \mathrm{H} \mathrm{NMR}, 500 \mathrm{MHz}, \mathrm{CDCl}_{3}$

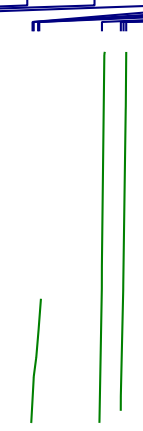


ㄷำ

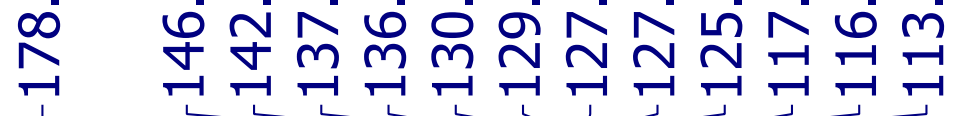

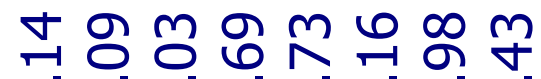

NN

I

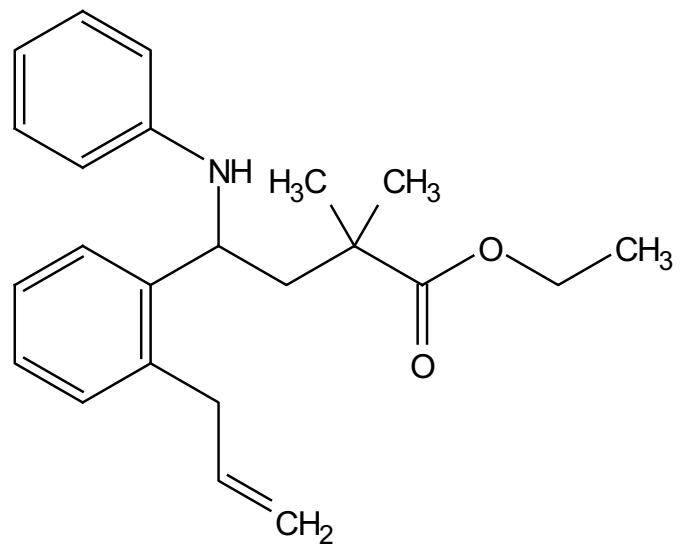

2h

${ }^{13} \mathrm{C} \mathrm{NMR}, 126 \mathrm{MHz}, \mathrm{CDCl}_{3}$ 


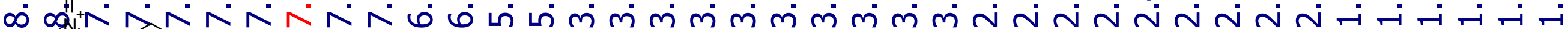
年<smiles>C=CC=C(CC)N(C)C(CC(C)(C)C(=O)OCC)c1ccccc1</smiles>

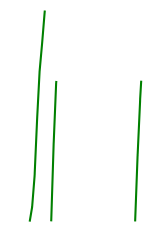

$2 \mathrm{i}$

${ }^{1} \mathrm{H} \mathrm{NMR}, 500 \mathrm{MHz}, \mathrm{CDCl}_{3}$
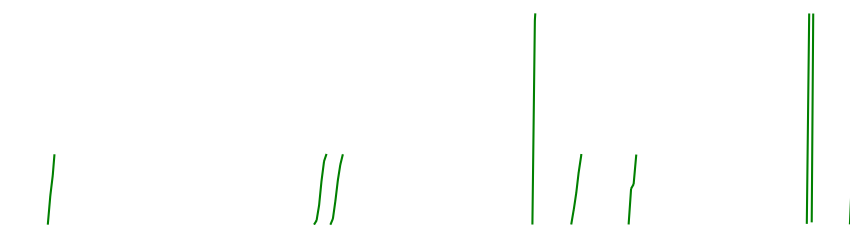


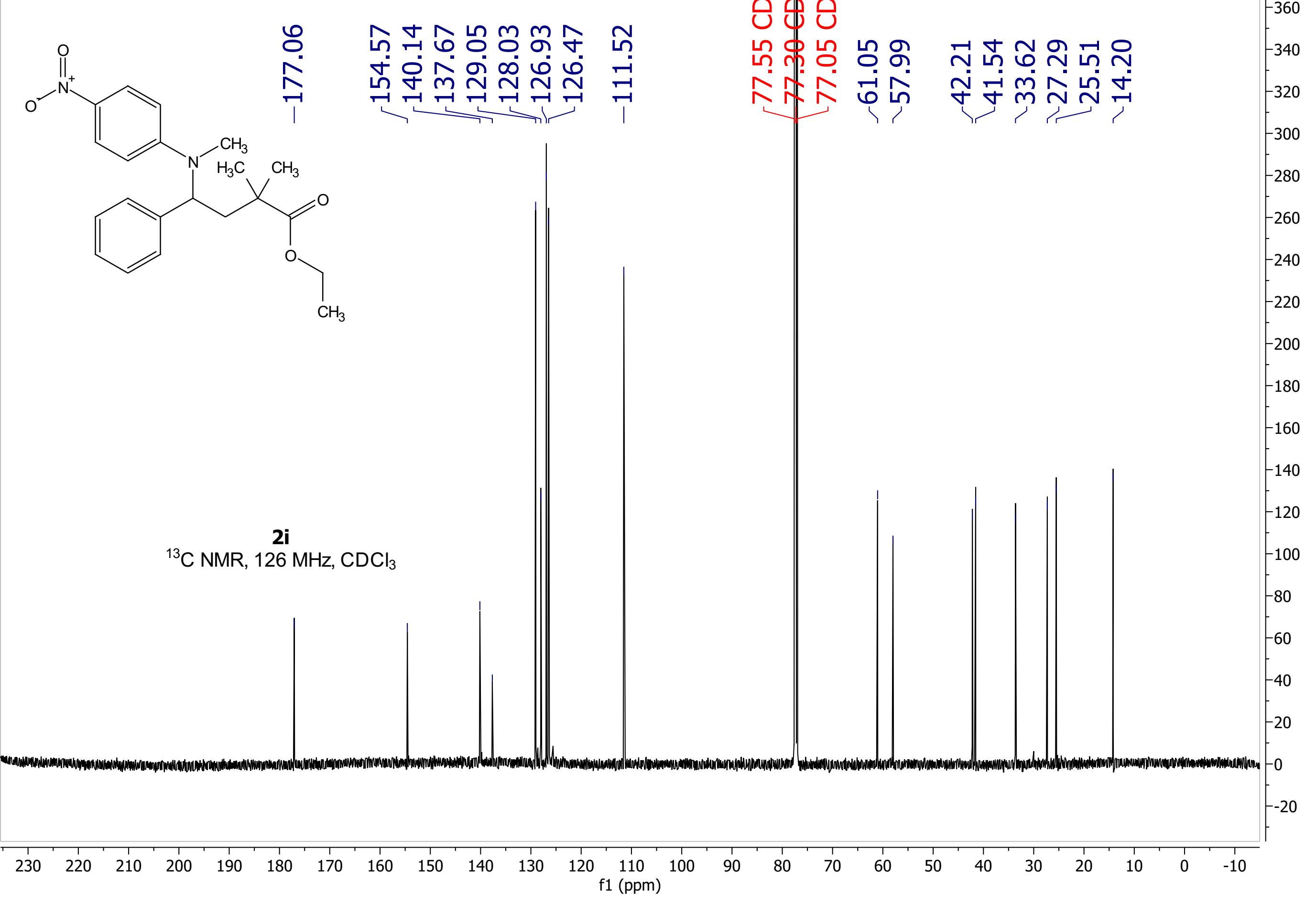


2j

1H NMR, $500 \mathrm{MHz}, \mathrm{CDCl} 3$

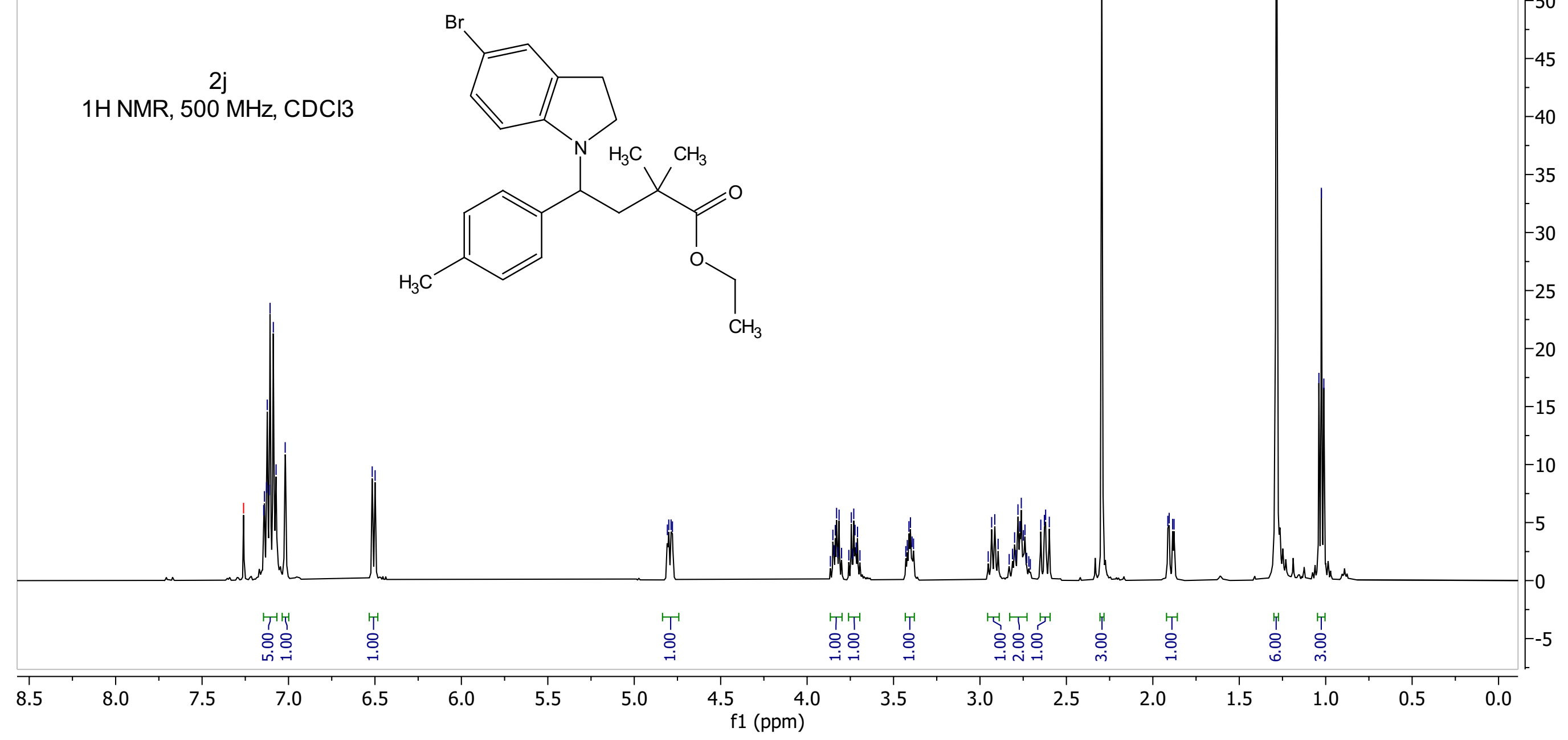


<smiles>CCOC(=O)C(C)(C)CC(c1ccc(Cl)cc1)N1CCc2cc(Br)ccc21</smiles>

2j

1H NMR, $500 \mathrm{MHz}, \mathrm{CDCl} 3$
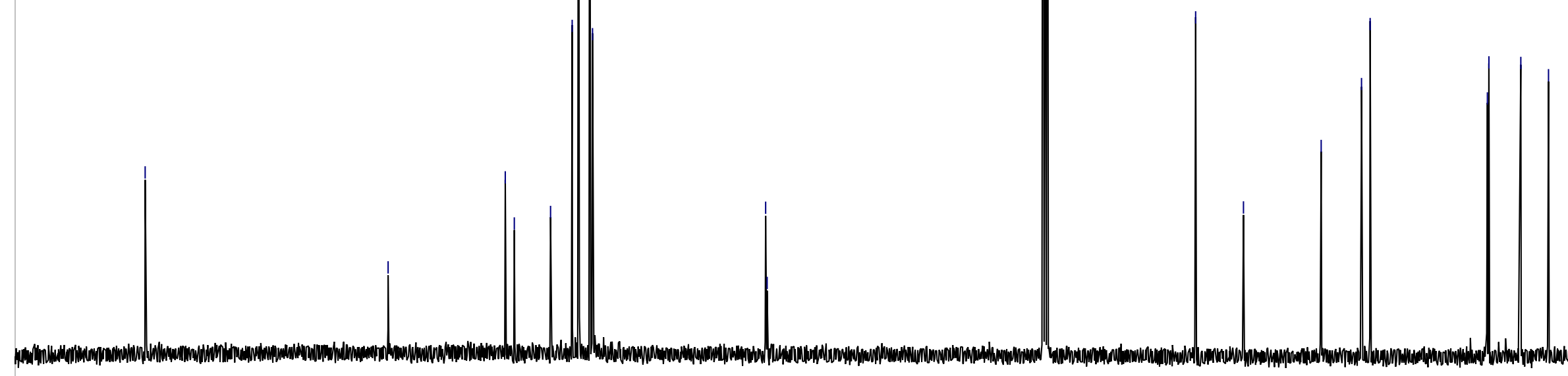


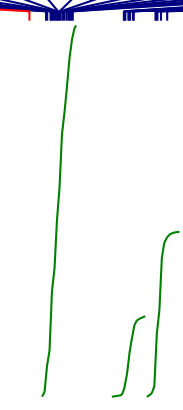

\section{$\int$}
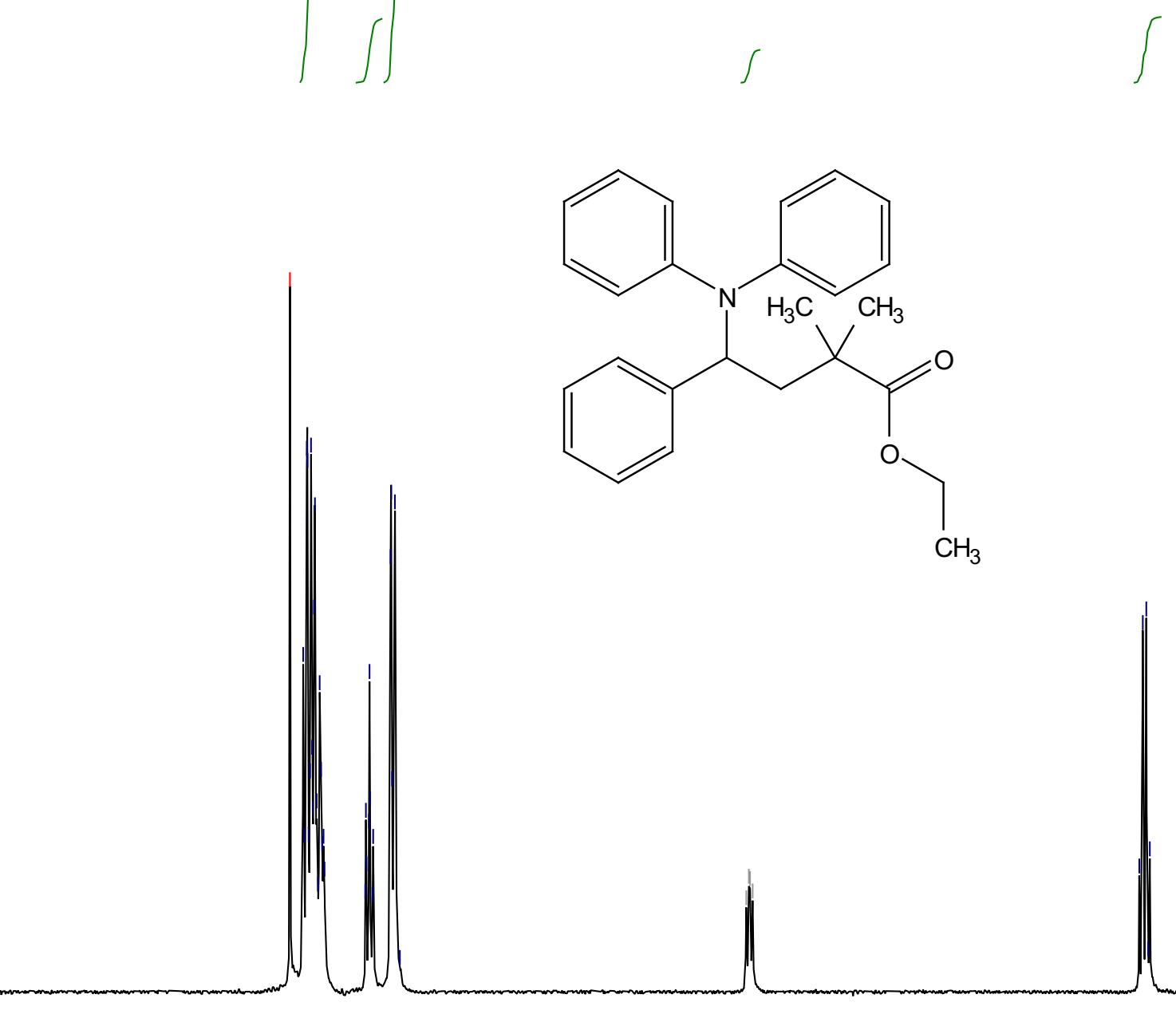

$2 \mathrm{k}$

$1 \mathrm{H} \mathrm{NMR,}, 500 \mathrm{MHz}, \mathrm{CDCl} 3$
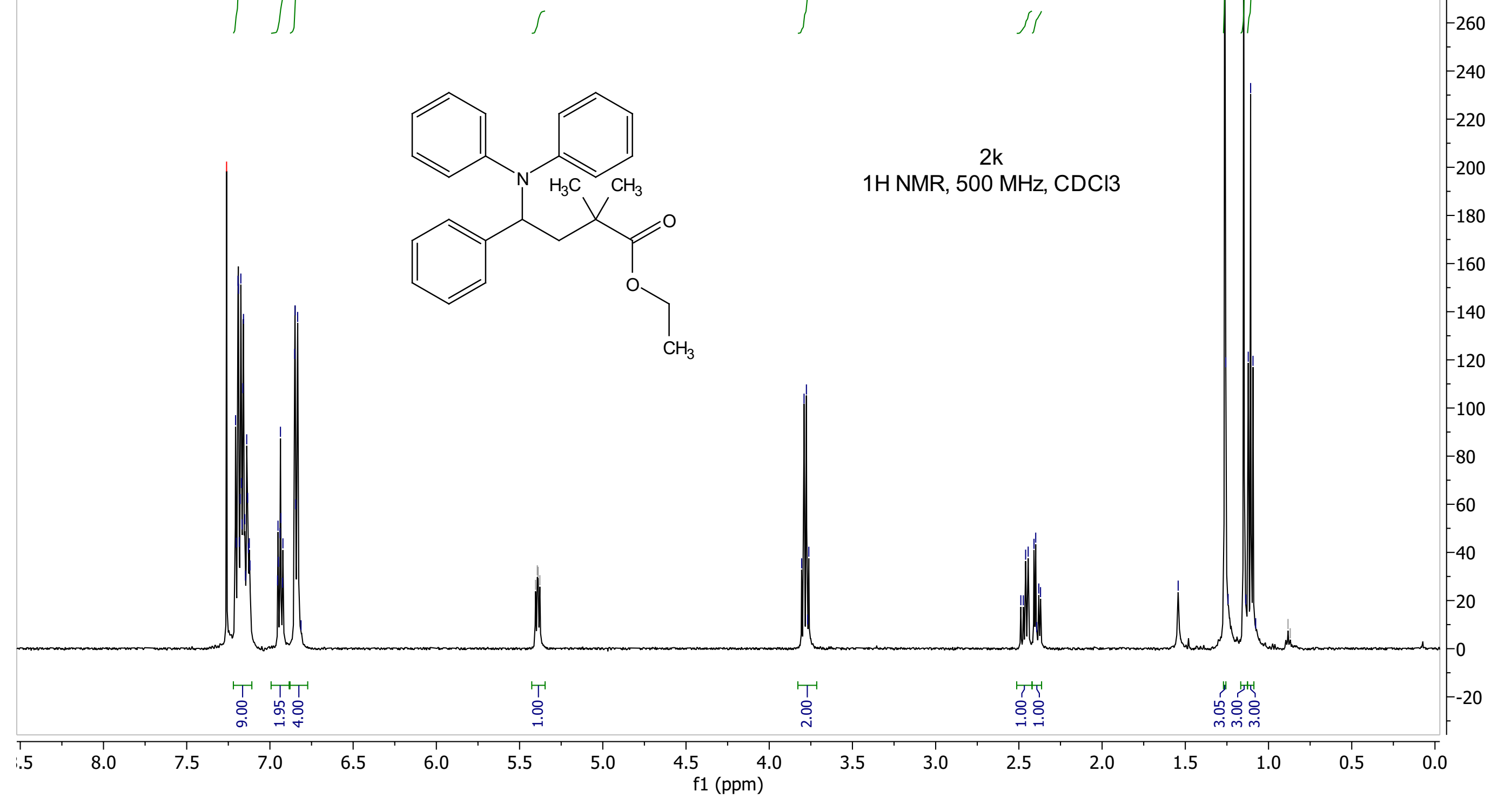
TLB-2-208-3-13C-32.10.fid

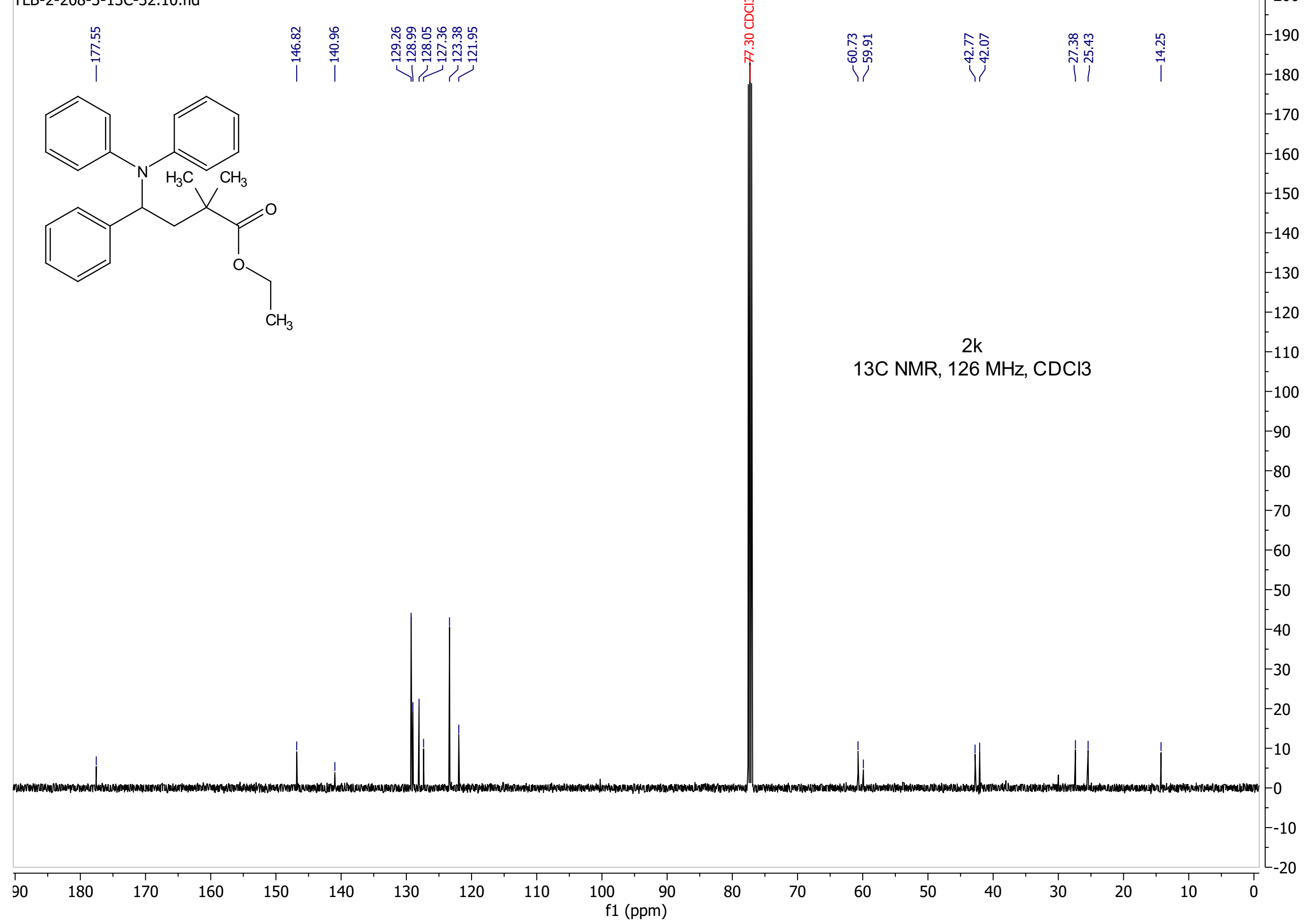


<smiles>CCOC(=O)C(C)(C)CC(c1ccc(OC)cc1)n1c2ccccc2c2ccccc21</smiles> 
<smiles>CCC(C)C</smiles><smiles>CCOC(=O)C(C)(C)CC(Nc1c(F)c(F)c(F)c(F)c1F)c1ccc(OC)cc1</smiles>

$2 \mathrm{~m}$

1H NMR, $500 \mathrm{MHz}, \mathrm{CDCl} 3$ 


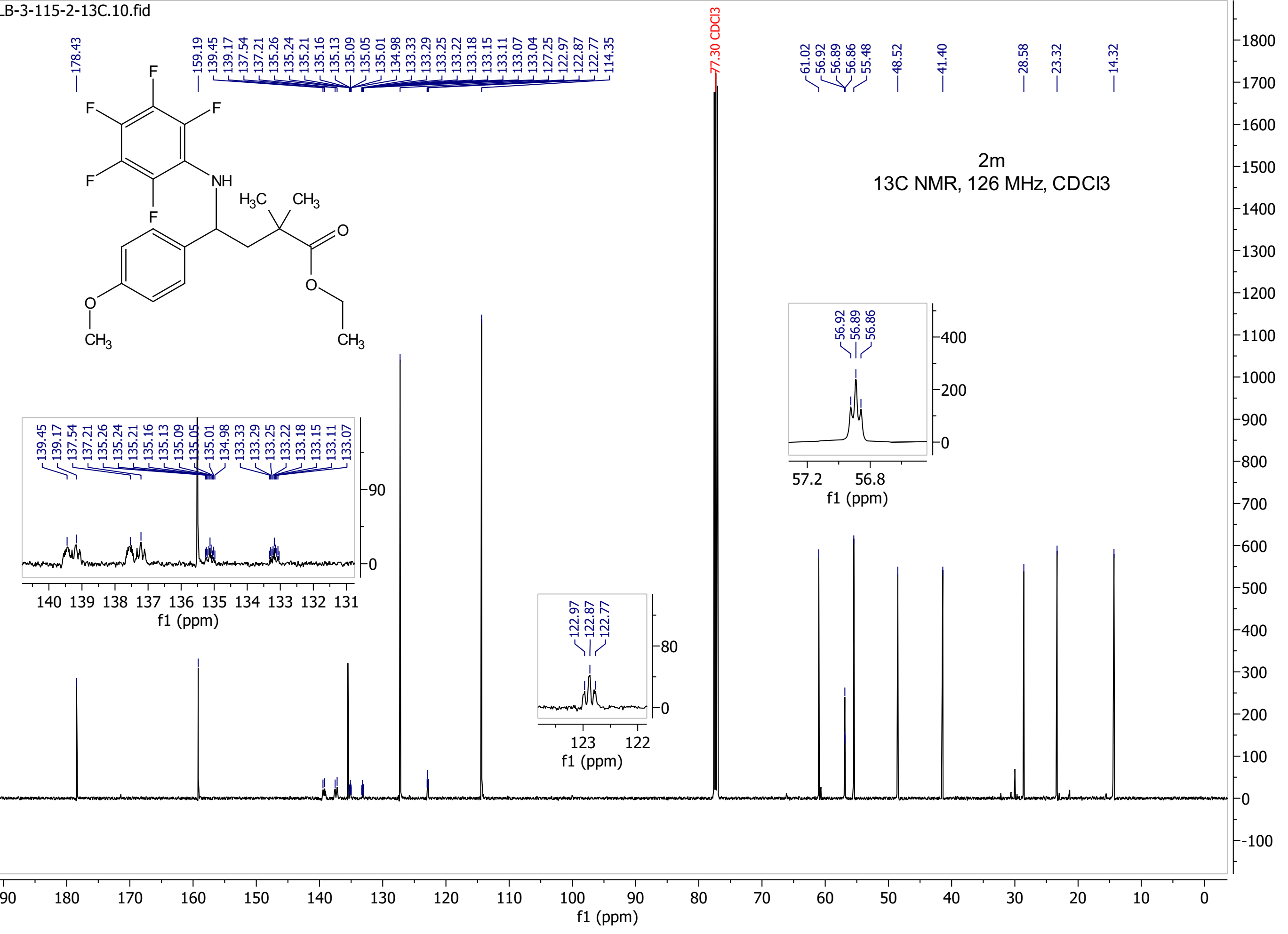




\section{$2 \mathrm{~m}$}

19F NMR, $471 \mathrm{MHz}, \mathrm{CDCl} 3$<smiles>CCOC(=O)C(C)(C)CC(Nc1c(F)c(F)c(F)c(F)c1F)c1ccc(OC)cc1</smiles> 


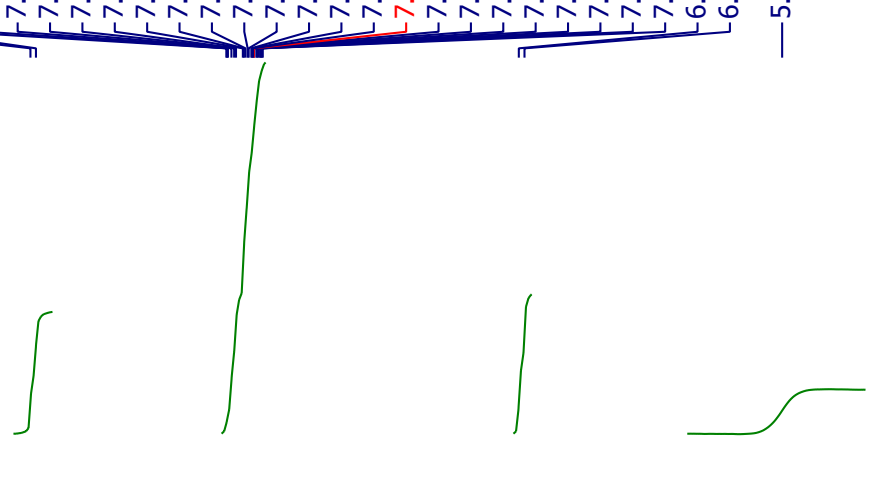

$2 n$

$1 \mathrm{H} \mathrm{NMR}, 500 \mathrm{MHz}, \mathrm{CDCl} 3$<smiles>CCOC(=O)C(C)(C)CC(Nc1ccc([N+](=O)[O-])cc1)c1ccccc1</smiles>

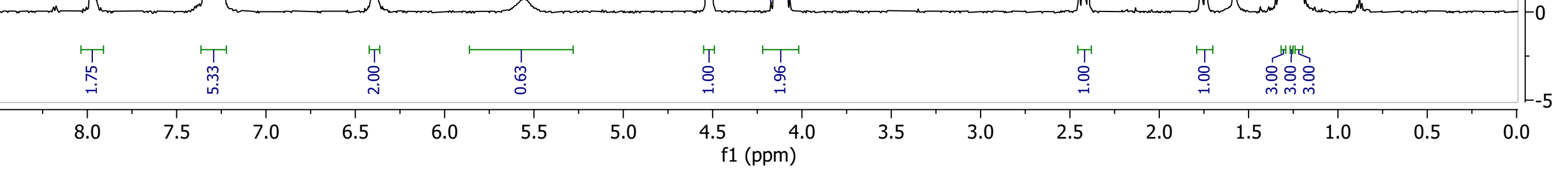


<smiles>CCOC(=O)C(C)(C)CC(Nc1ccc([N+](=O)[O-])cc1)c1ccccc1</smiles>

13C NMR, $126 \mathrm{MHz}, \mathrm{CDCl} 3$

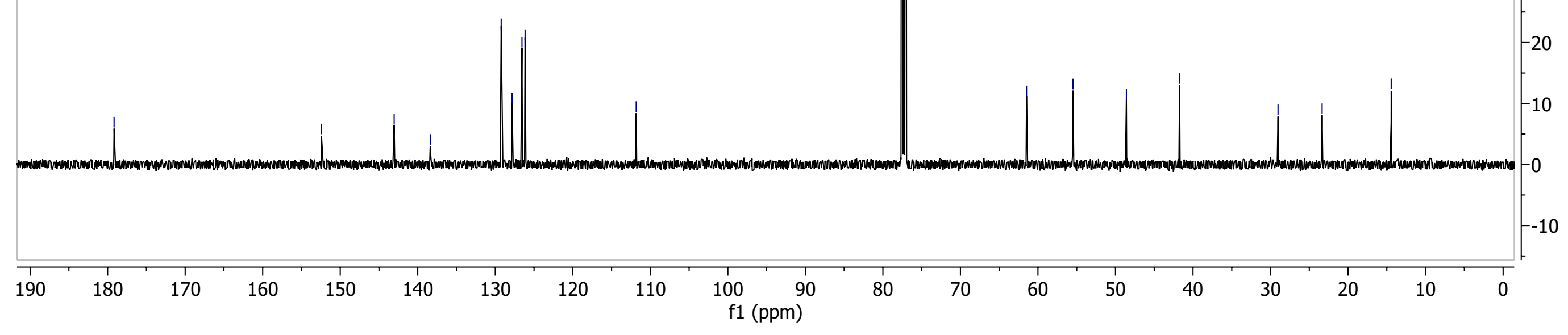


m

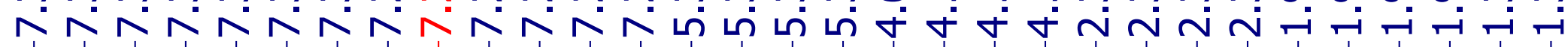

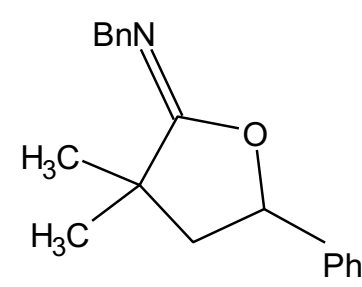

20

${ }^{1} \mathrm{H} \mathrm{NMR}, 500 \mathrm{MHz}, \mathrm{CDCl}_{3}$
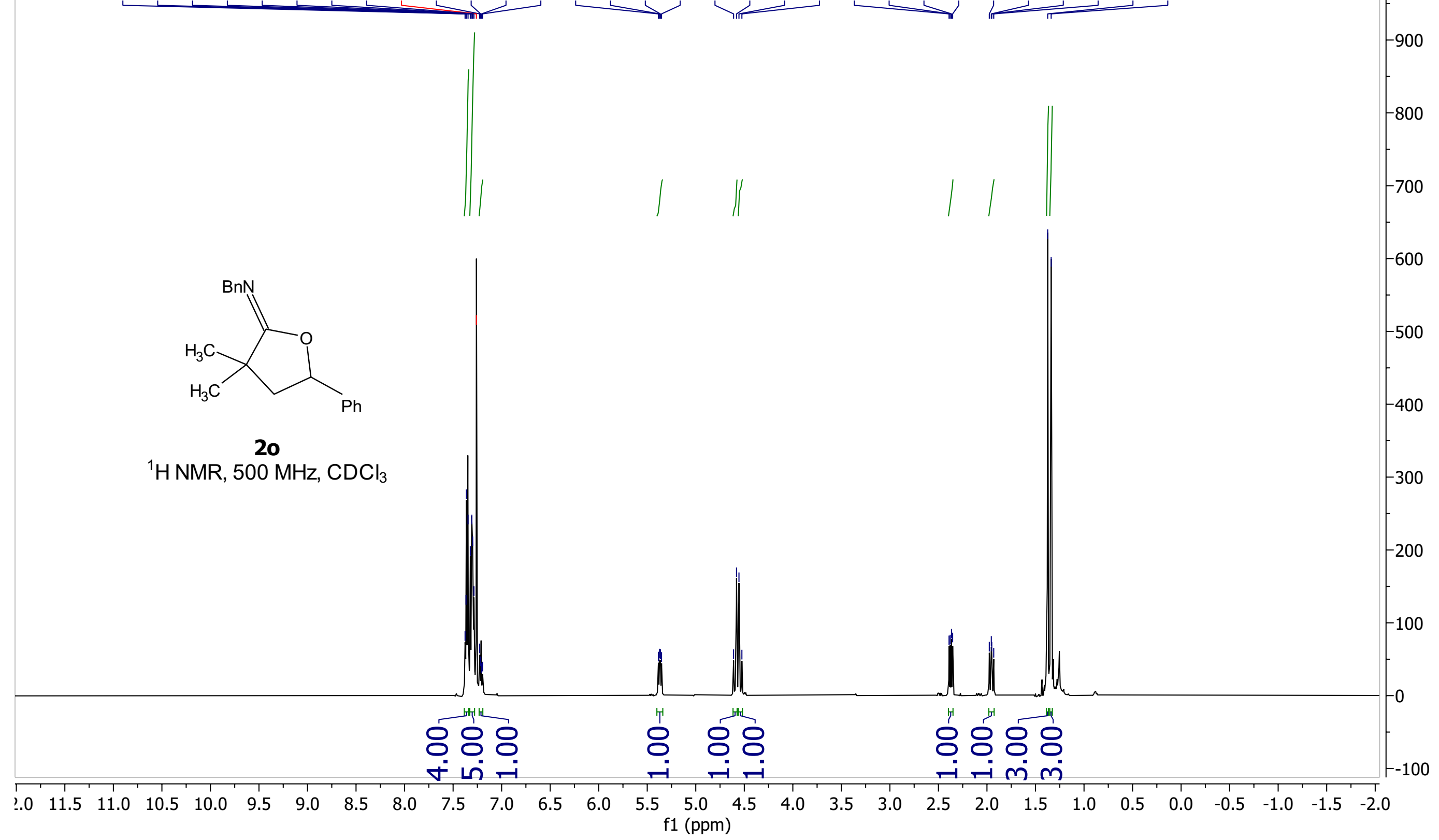


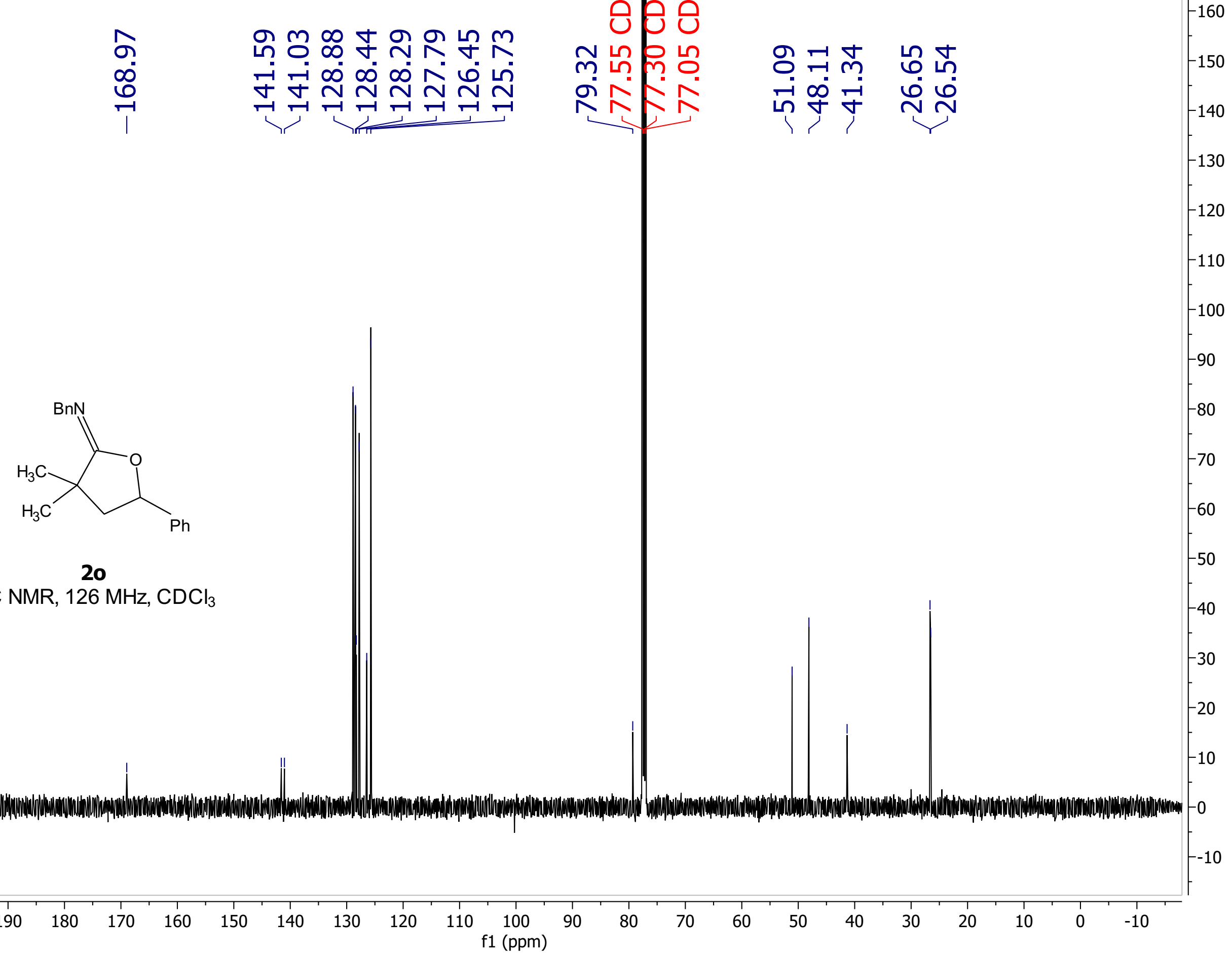



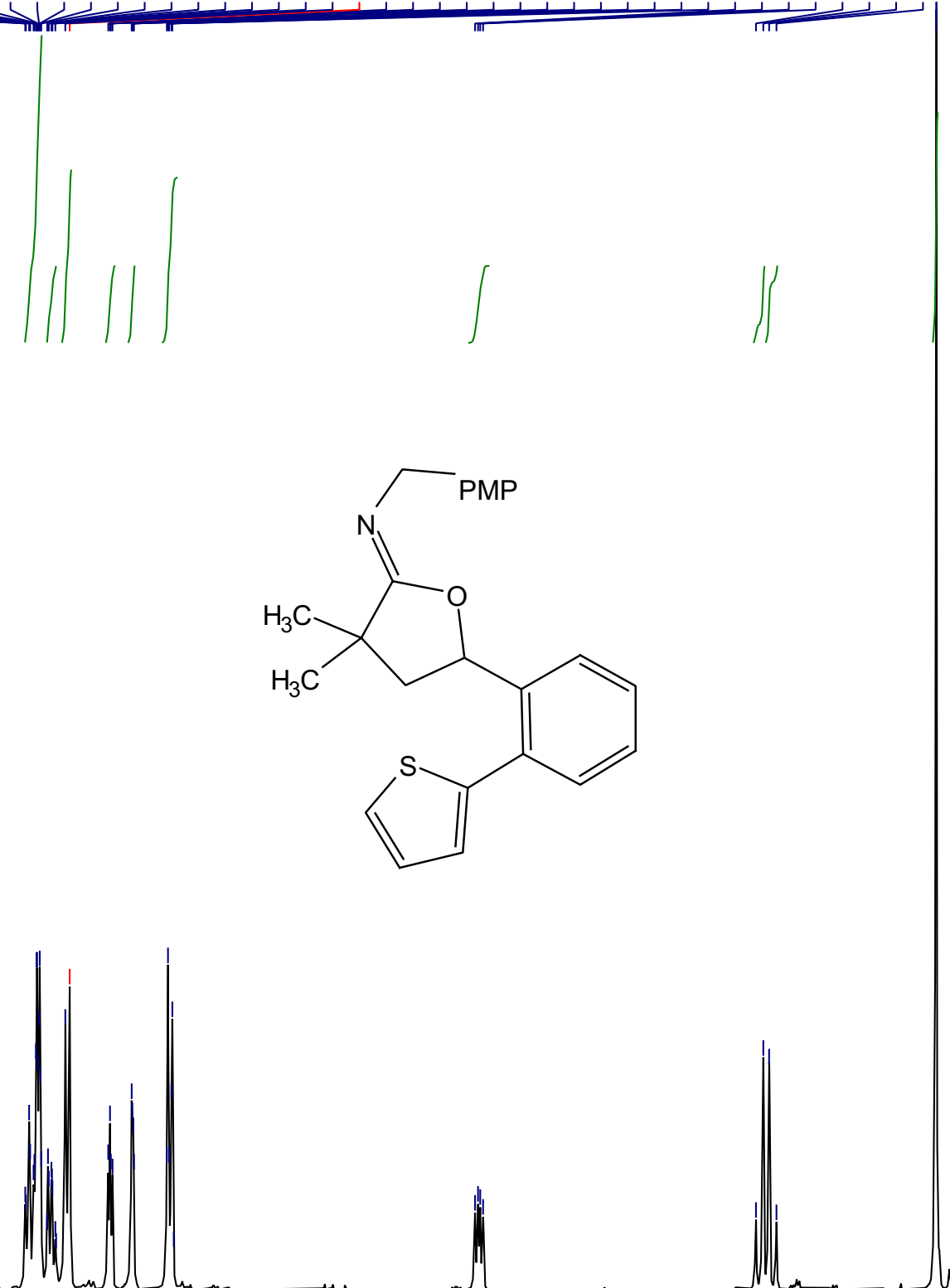

$2 p$

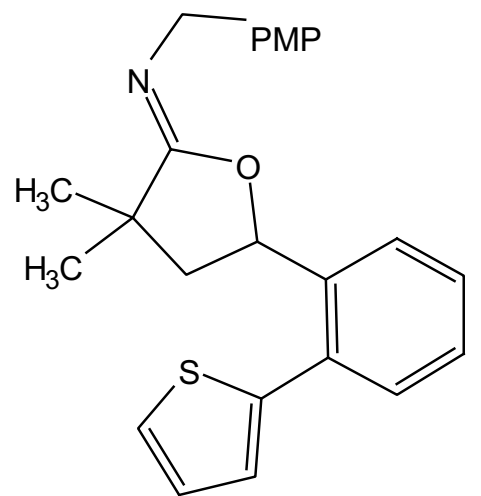

1H NMR, $500 \mathrm{MHz}, \mathrm{CDCl} 3$

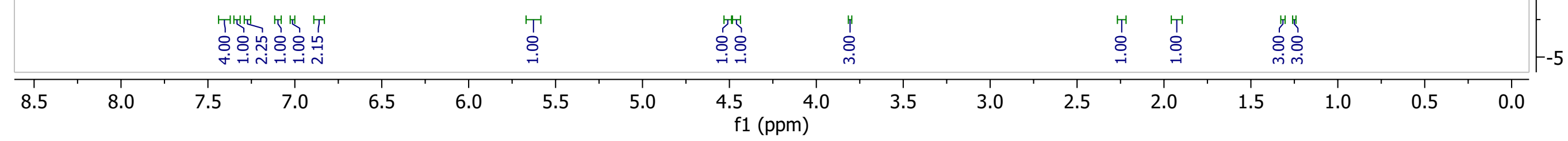




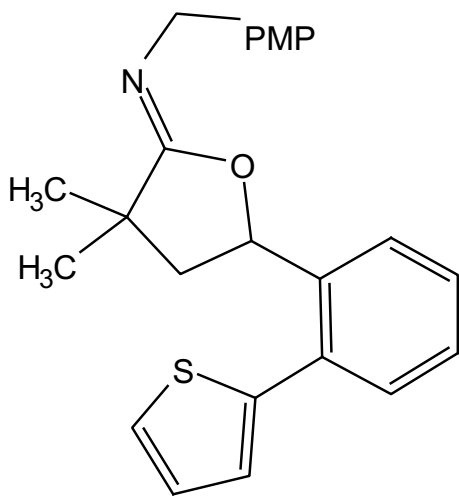

$2 \mathrm{p}$

13C NMR, $126 \mathrm{MHz}$, $\mathrm{CDCl} 3$ 
6

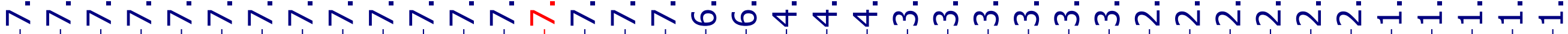
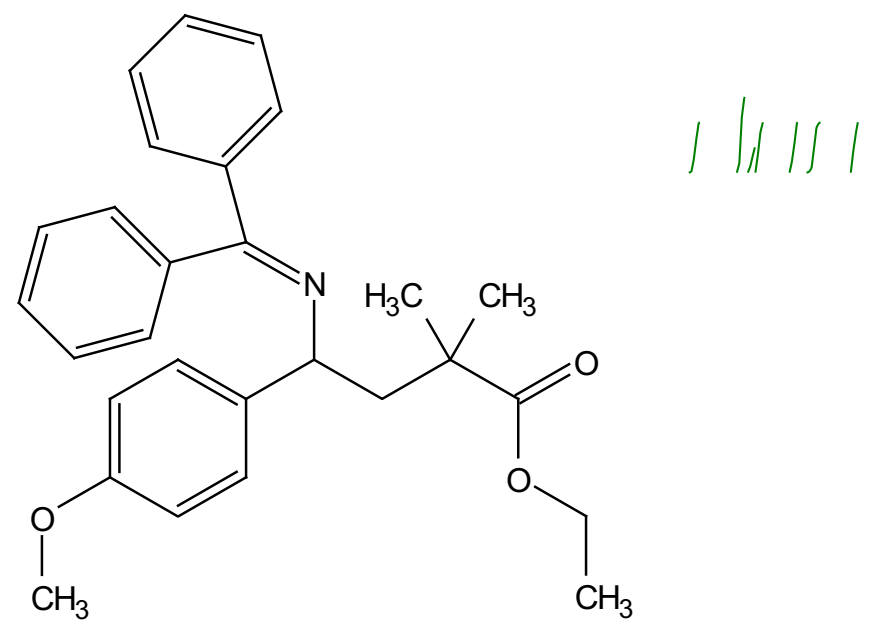

$2 q$

${ }^{1} \mathrm{H} \mathrm{NMR}, 500 \mathrm{MHz}, \mathrm{CDCl}_{3}$
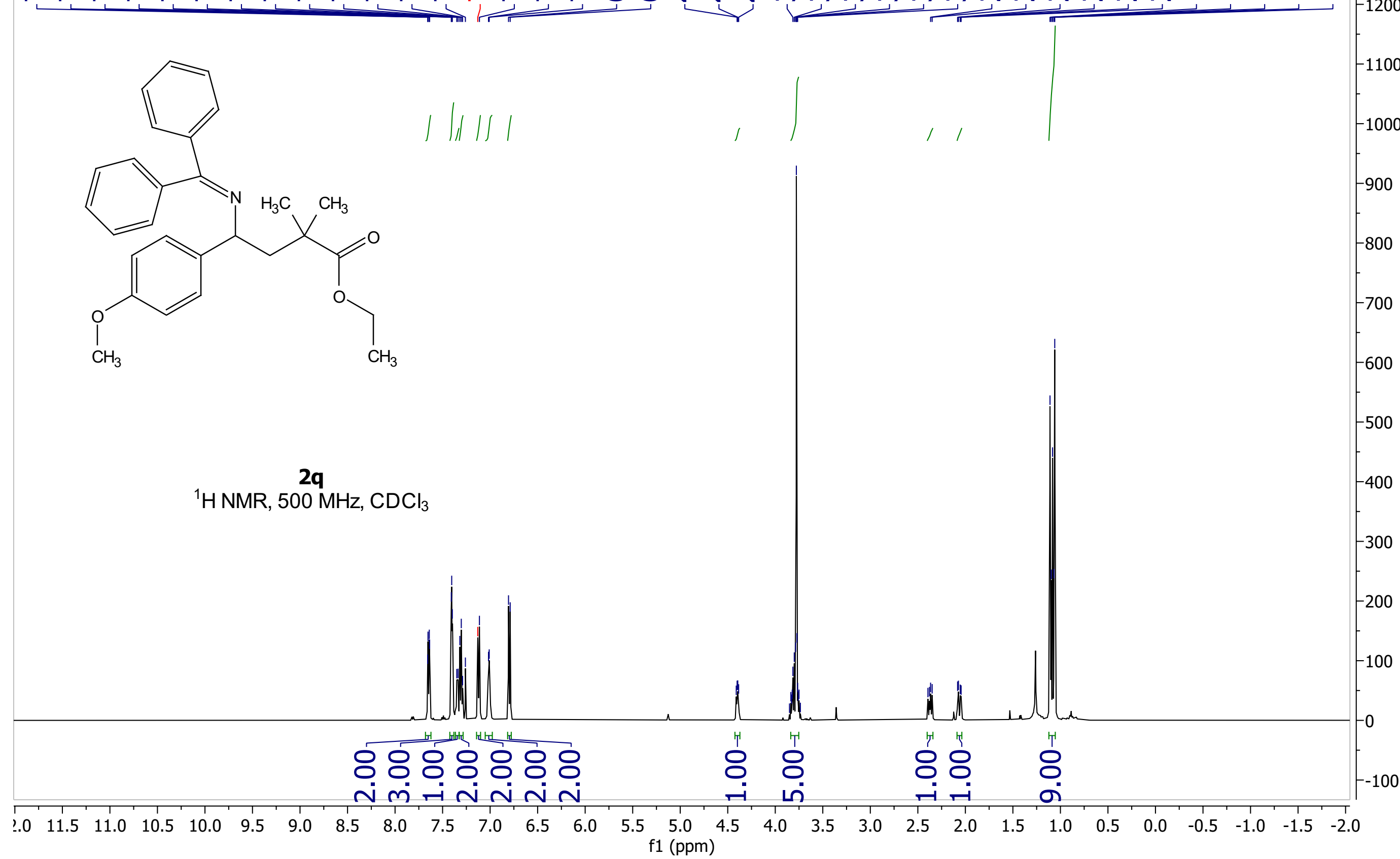
б ซ N

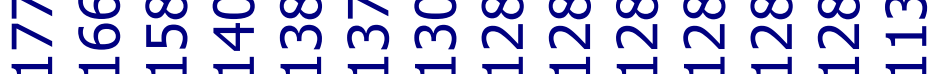

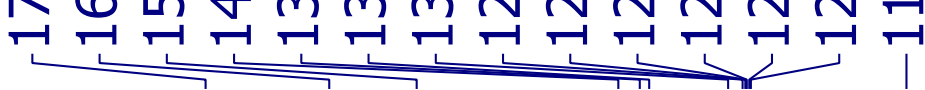

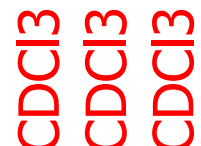

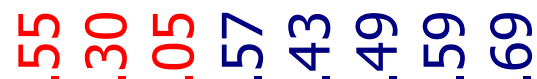
NNNmo vo

№
กNำ

ต

N

${ }^{13} \mathrm{C} \mathrm{NMR}, 126 \mathrm{MHz}, \mathrm{CDCl}_{3}$
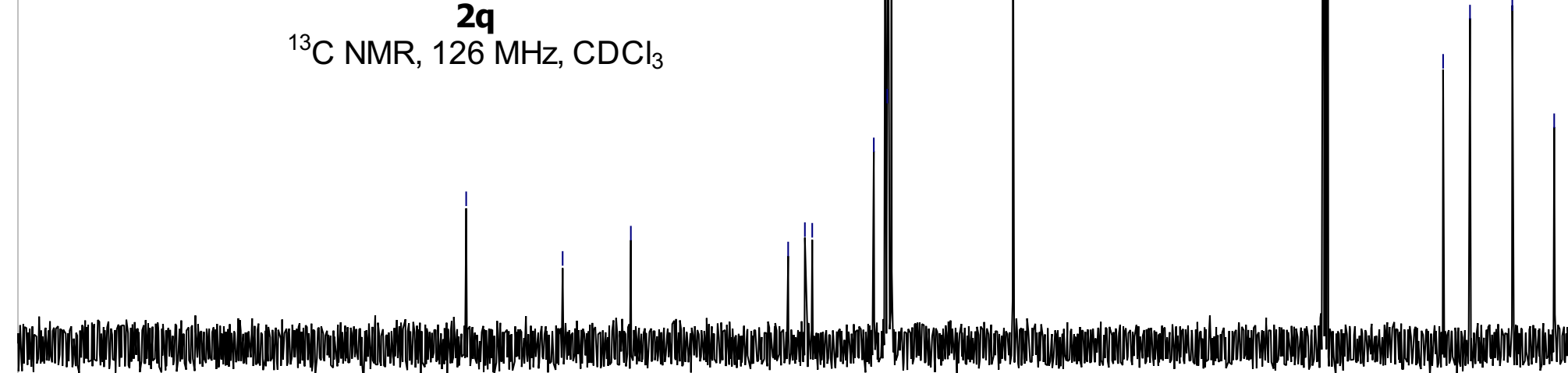


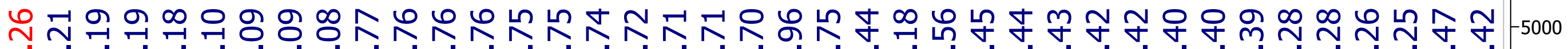

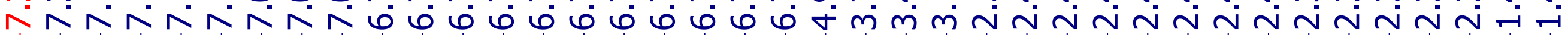
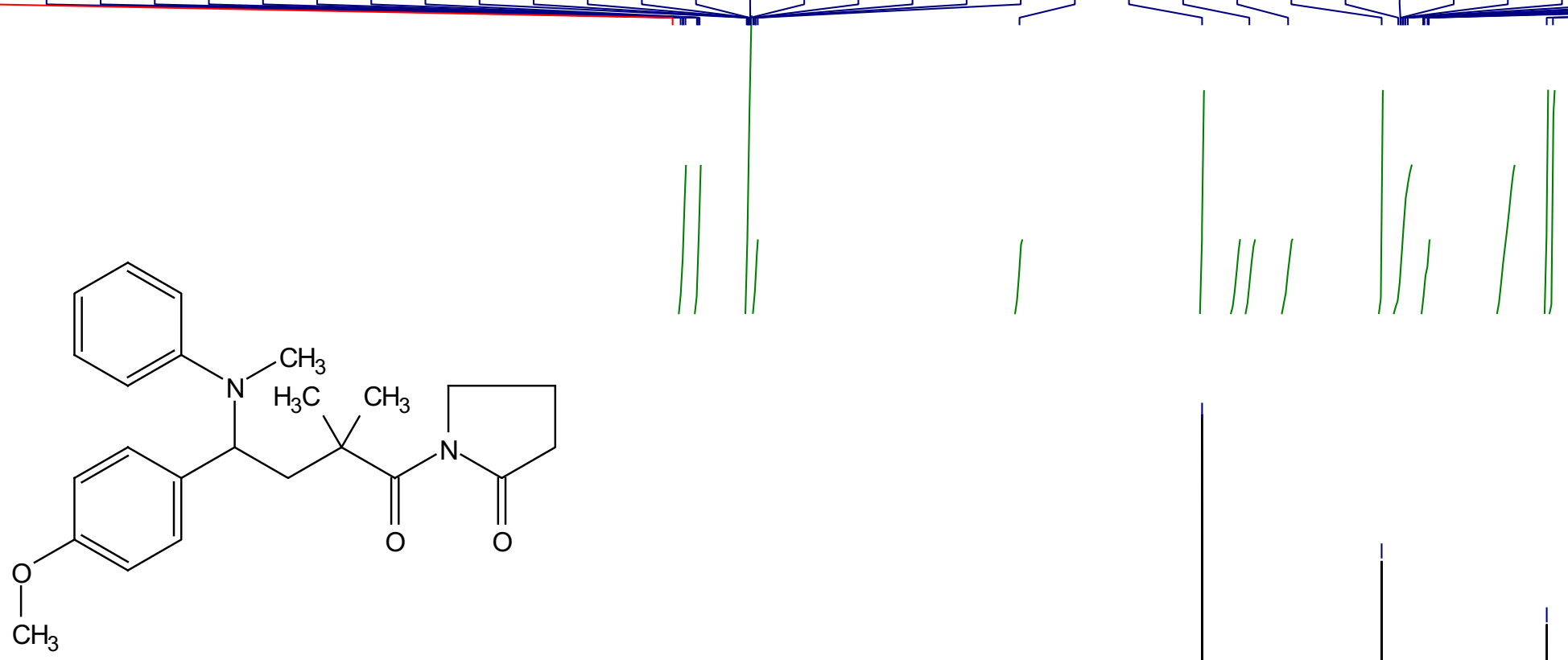

$2 r$

${ }^{1} \mathrm{HNMR}, 500 \mathrm{MHz}, \mathrm{CDCl}_{3}$

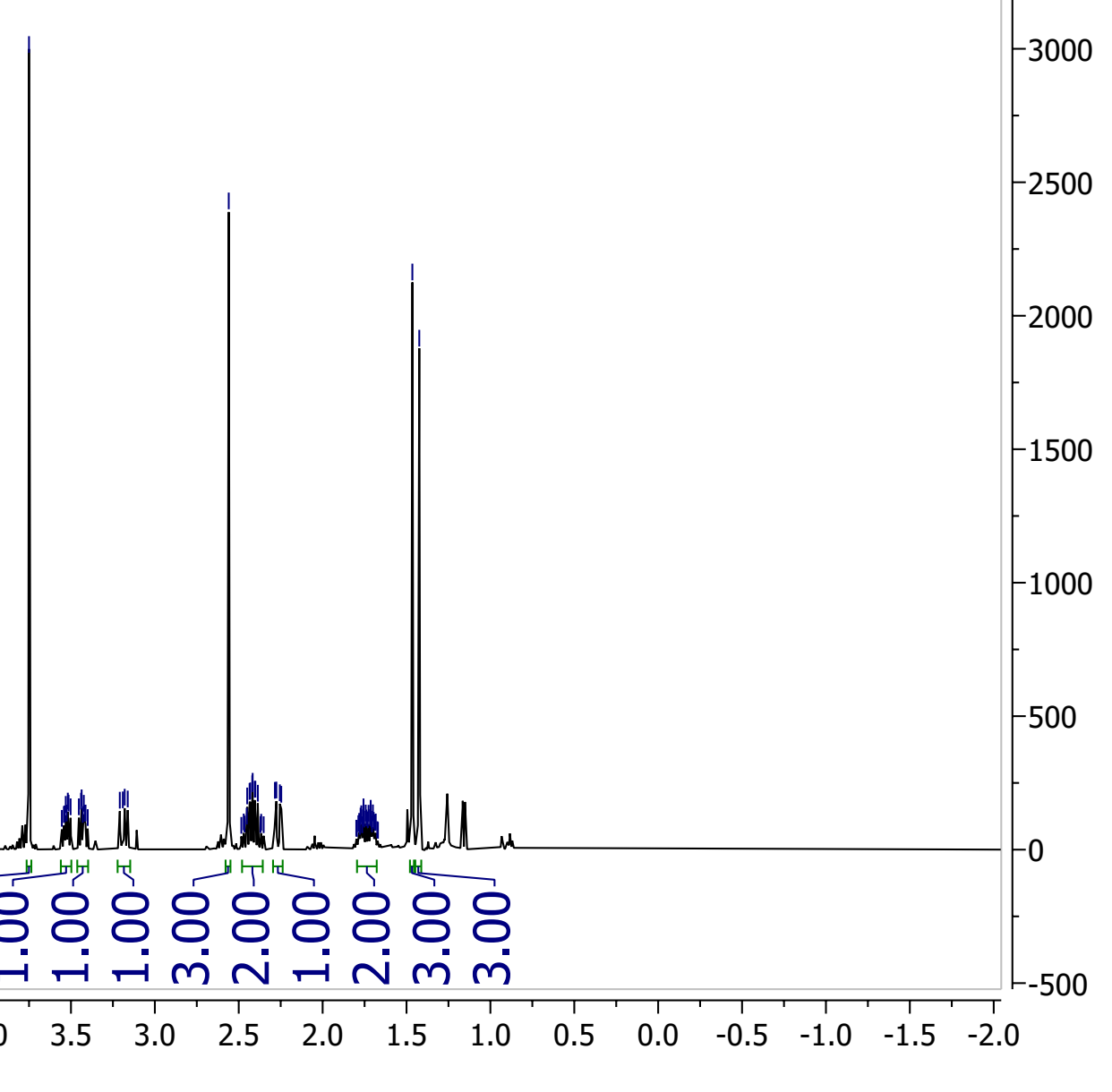




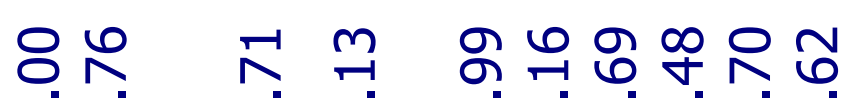

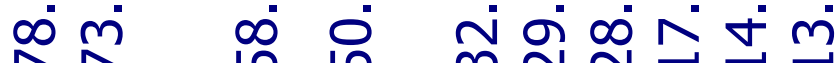

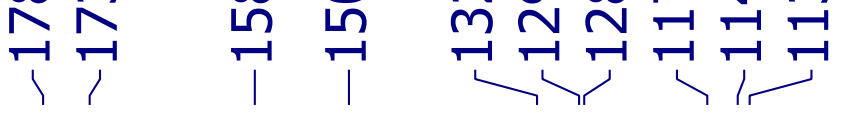

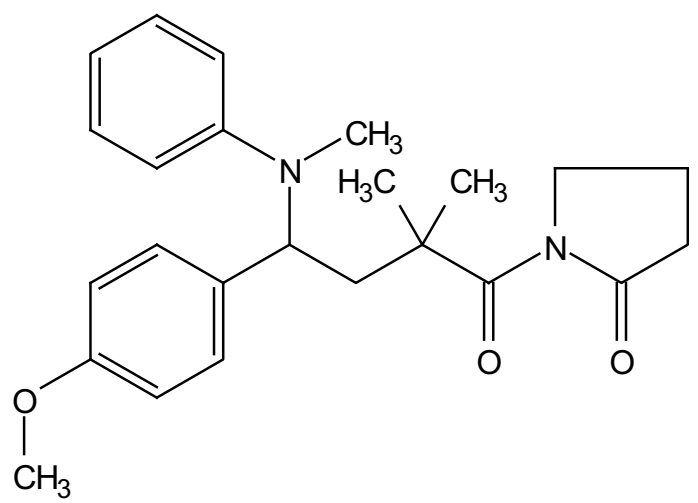

$2 r$

${ }^{13} \mathrm{C} \mathrm{NMR}, 126 \mathrm{MHz}, \mathrm{CDCl}_{3}$

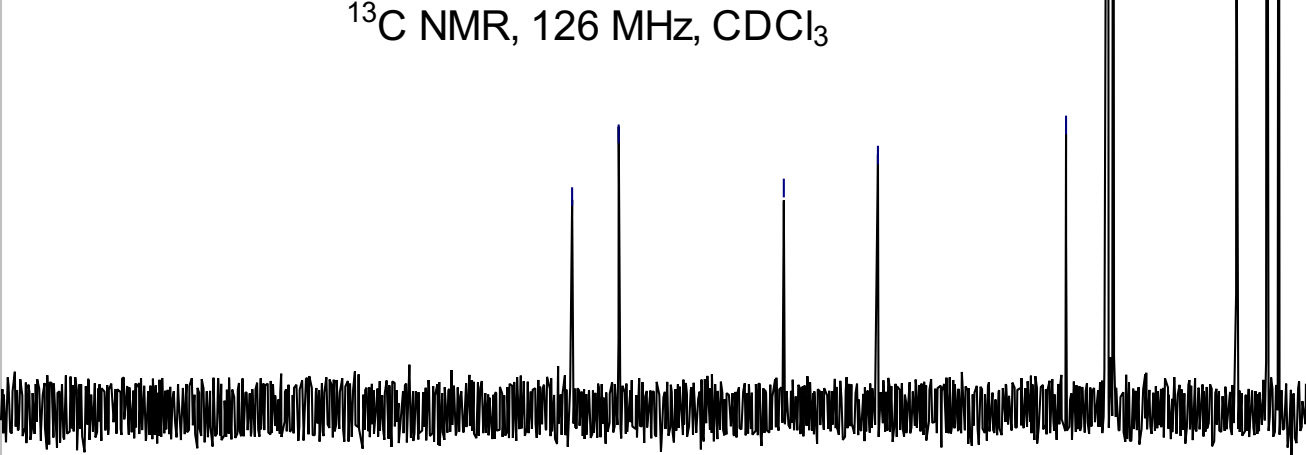

๖ํํำ

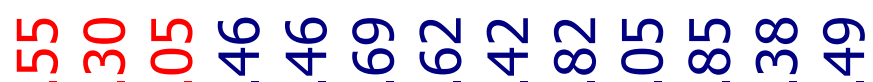
손

YNN

$190 \quad 180$

150

$120 \quad 110$

90

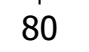

70

60
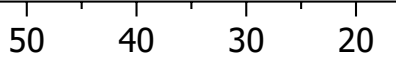


\section{TLB-2-221-1Ppactualpab}

STANDARD PROTON PARAMETERS

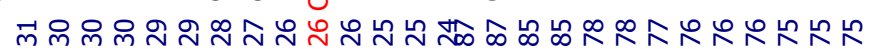

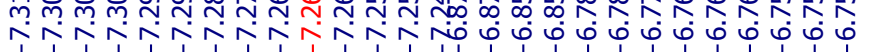

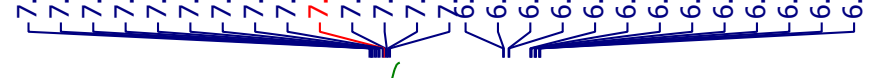

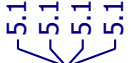

$\iint$

2s

1H NMR, $500 \mathrm{MHz}, \mathrm{CDCl} 3$
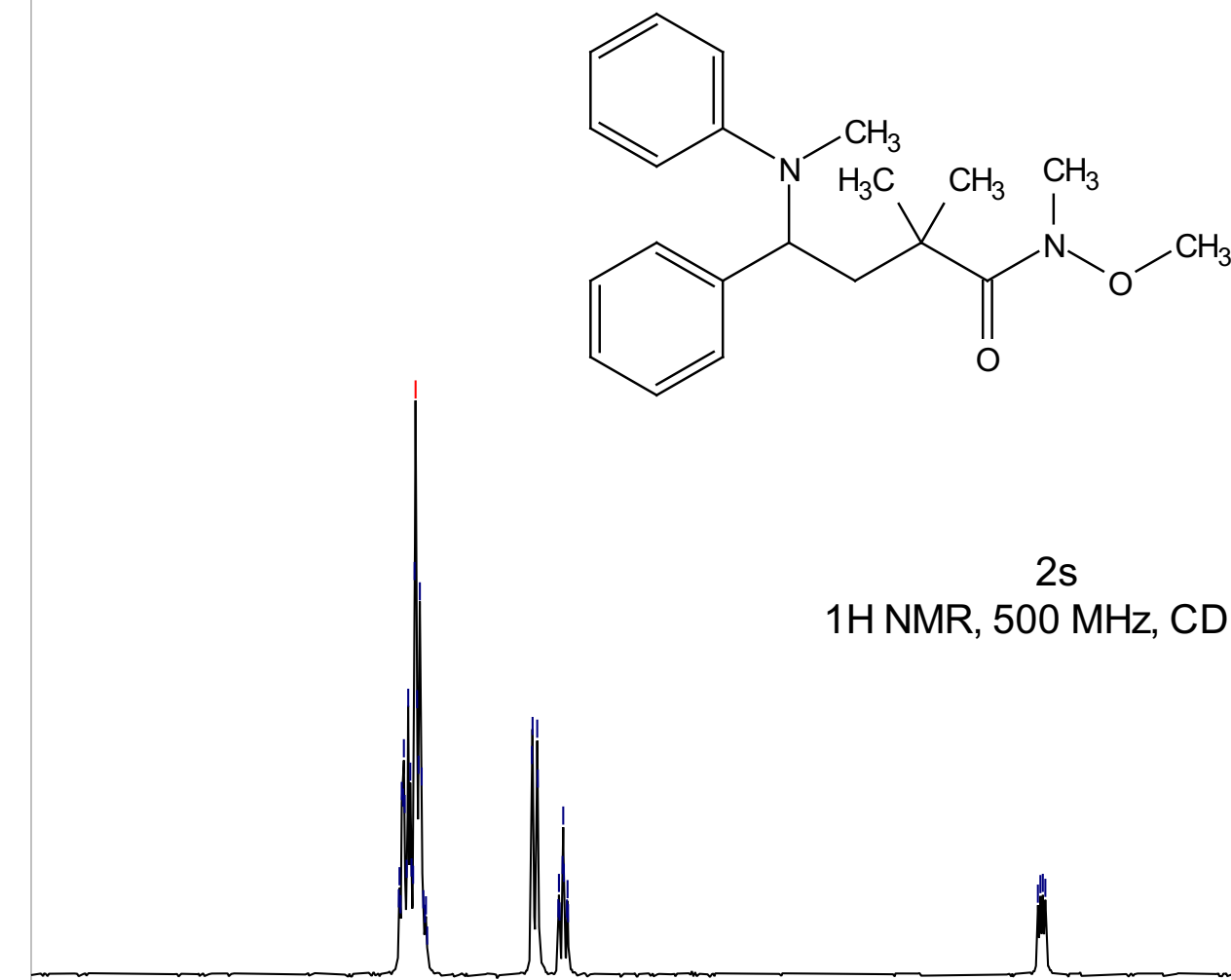

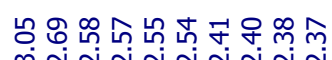

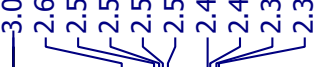

ij

85
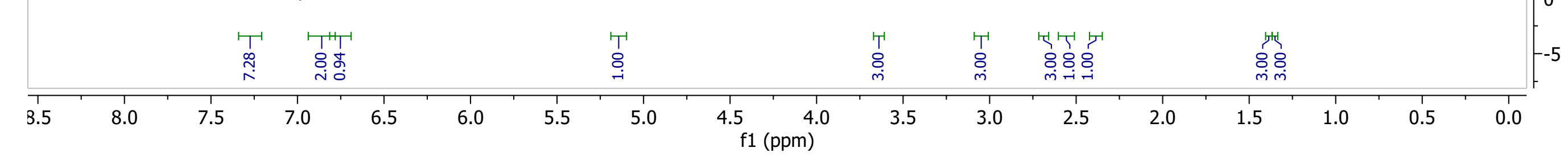


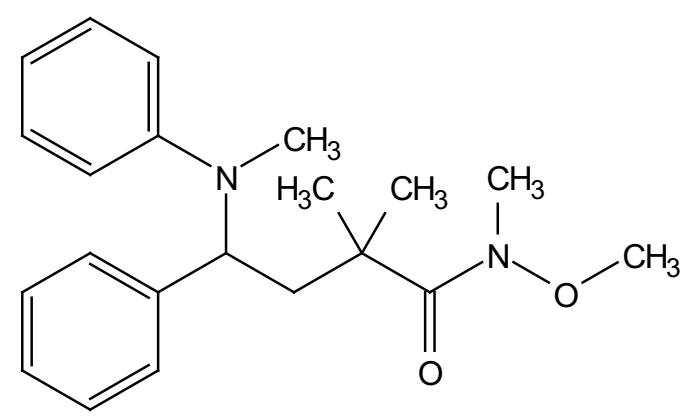

2s

13C NMR, $126 \mathrm{MHz}, \mathrm{CDCl} 3$ 

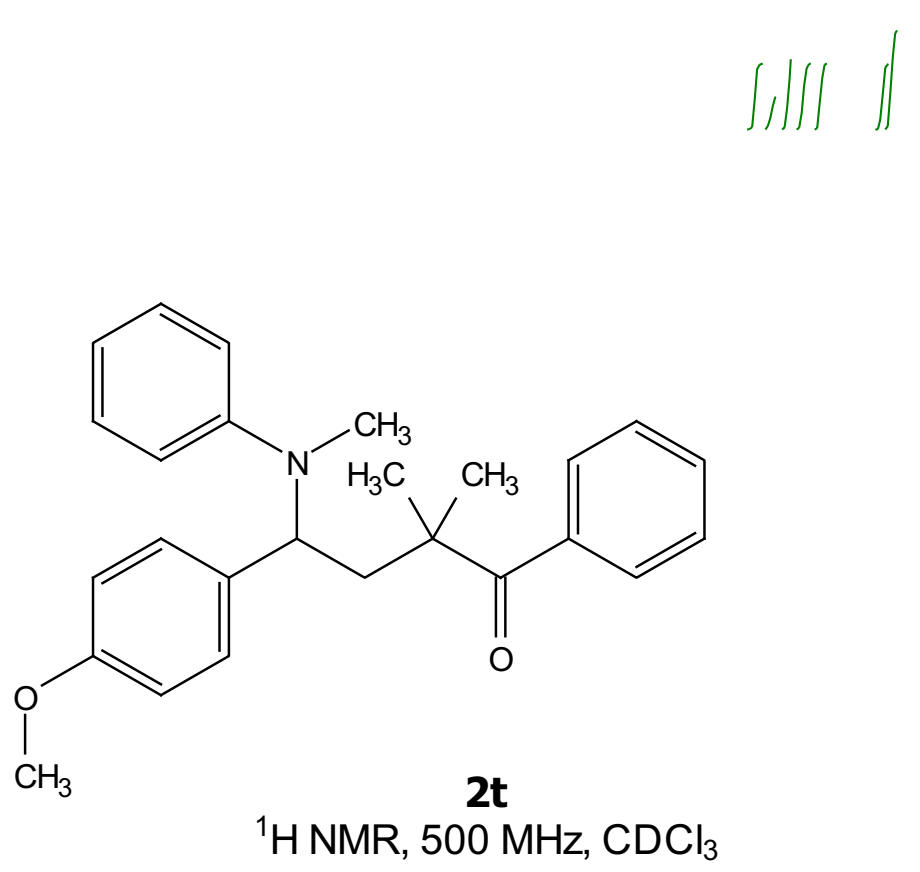

${ }^{1} \mathrm{HNMR}, 500 \mathrm{MHz}, \mathrm{CDCl}_{3}$

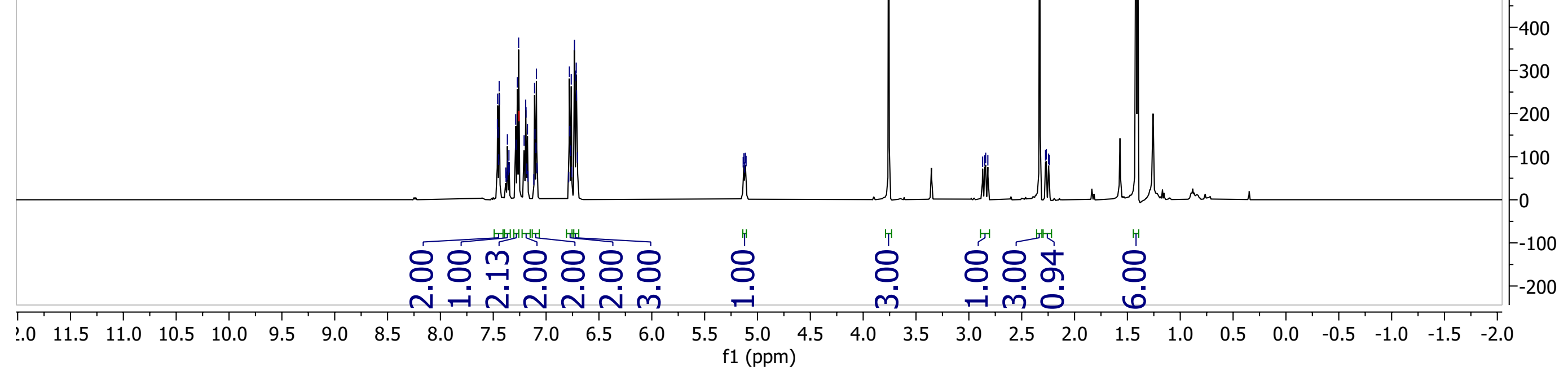




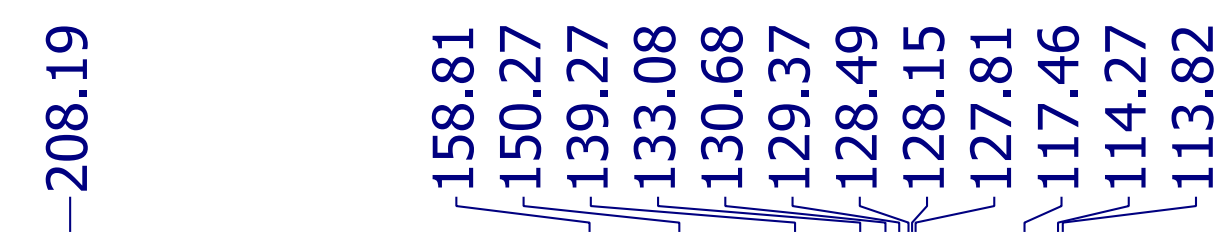

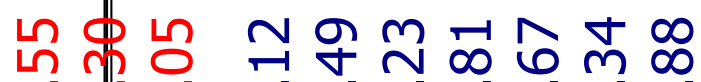

$\therefore N \infty$ ก
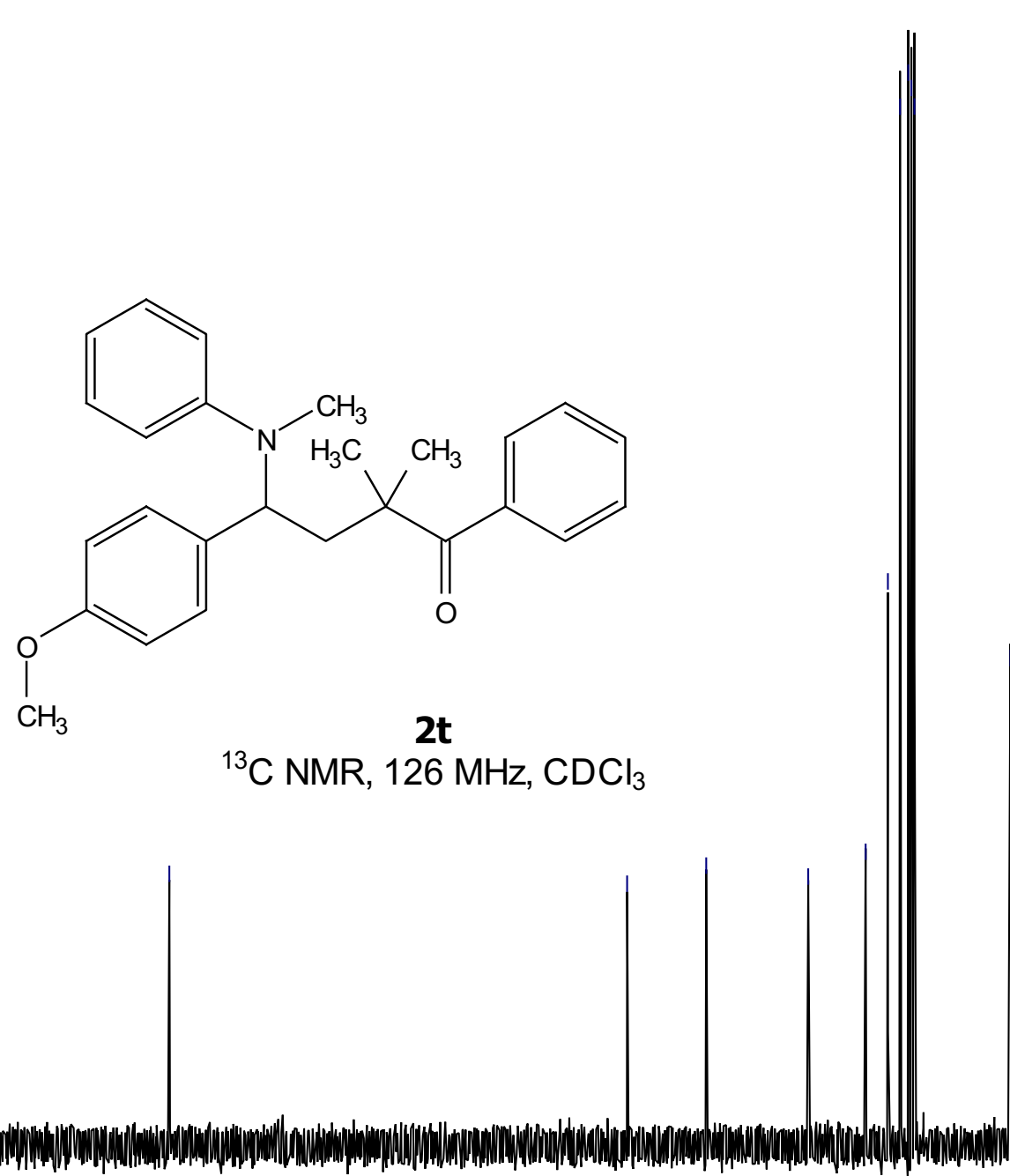

${ }^{13} \mathrm{C} \mathrm{NMR}, 126 \mathrm{MHz}, \mathrm{CDCl}_{3}$
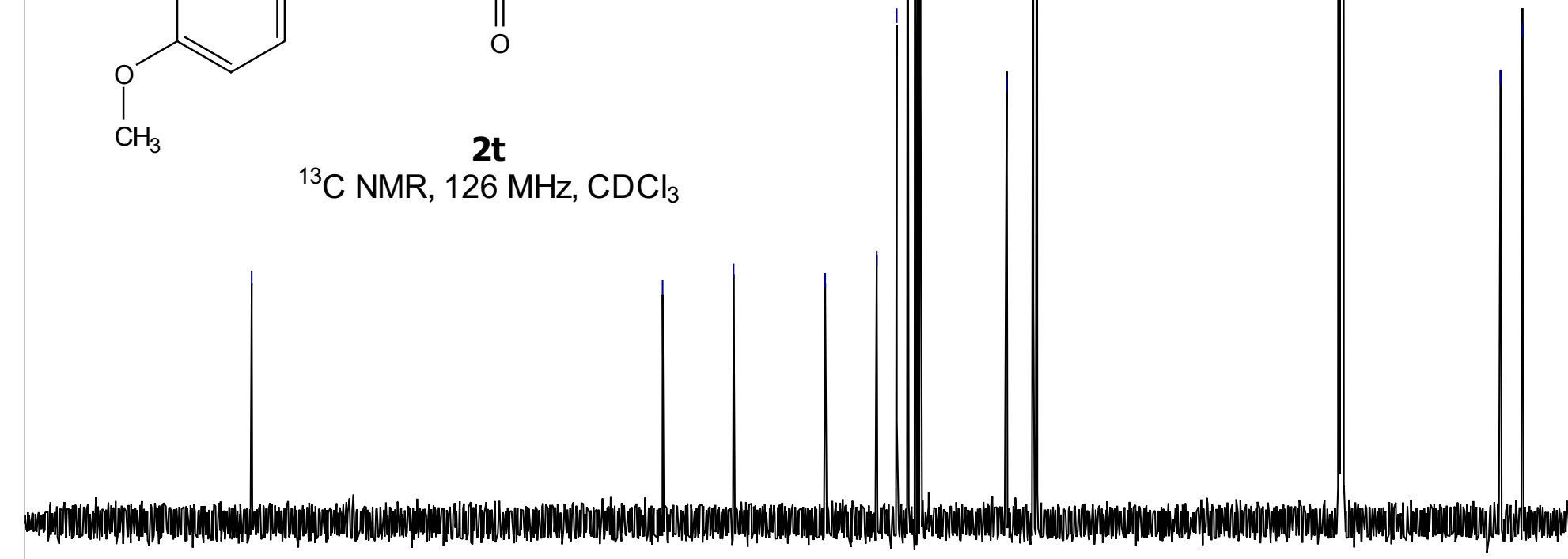

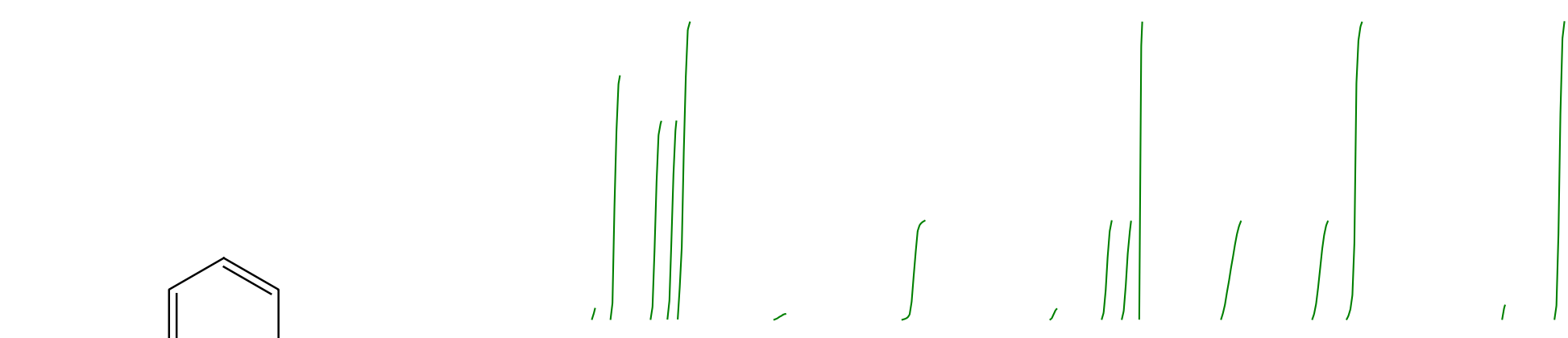<smiles>C/C=C\N(C)C(CC(F)(F)C(=O)OCC)c1ccc(OC)cc1</smiles>

$2 u$

${ }^{1} \mathrm{H} \mathrm{NMR}, 500 \mathrm{MHz}, \mathrm{CDCl}_{3}$

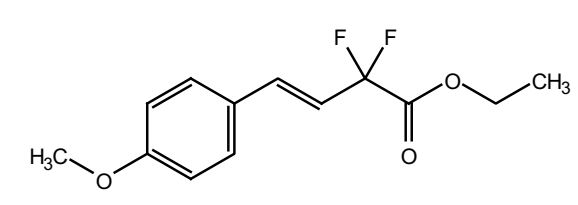

$-1000$ 


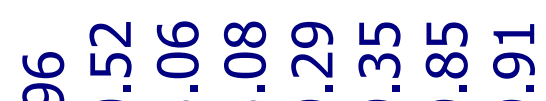

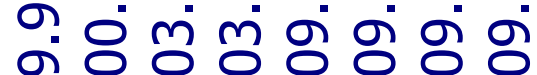

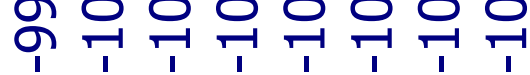
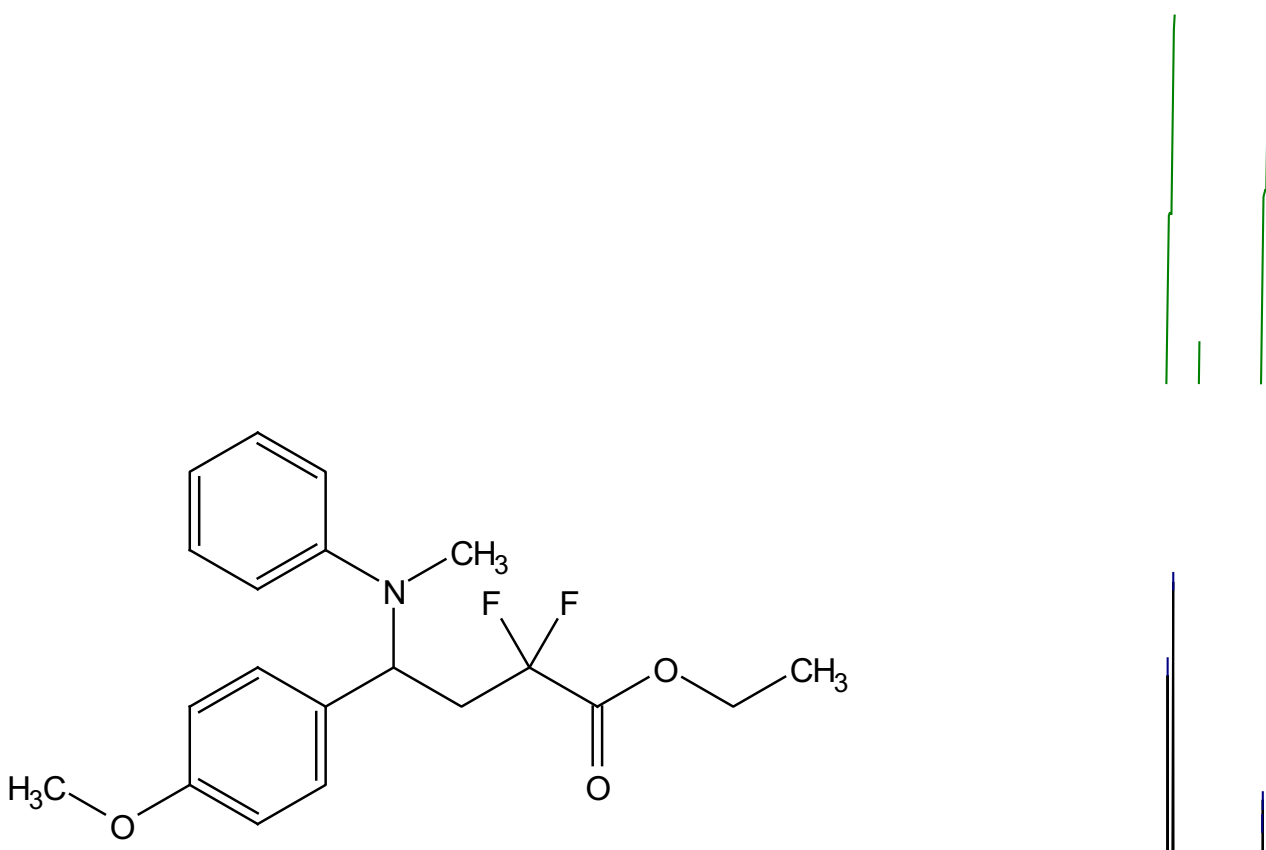

$2 \mathbf{u}$

${ }^{19} \mathrm{~F} \mathrm{NMR}, 470 \mathrm{MHz}, \mathrm{CDCl}_{3}$

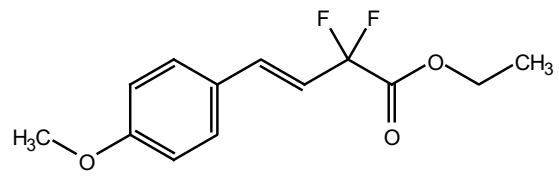

(6\% impurity)

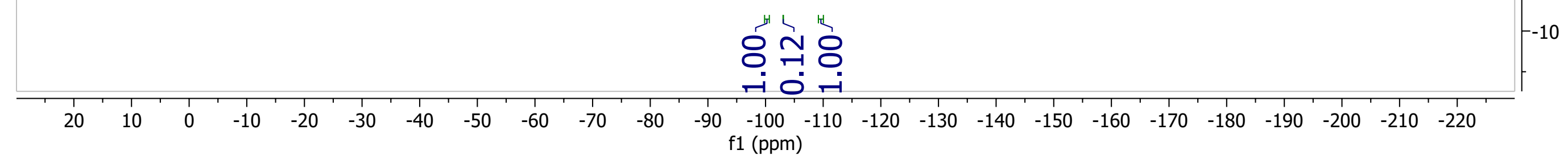




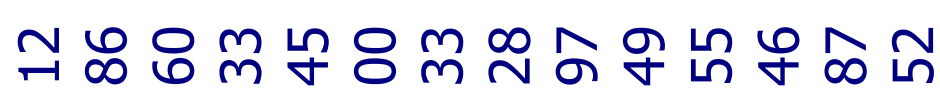

ナm m

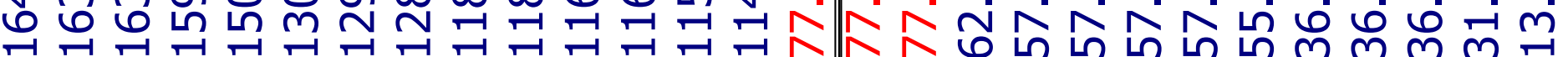

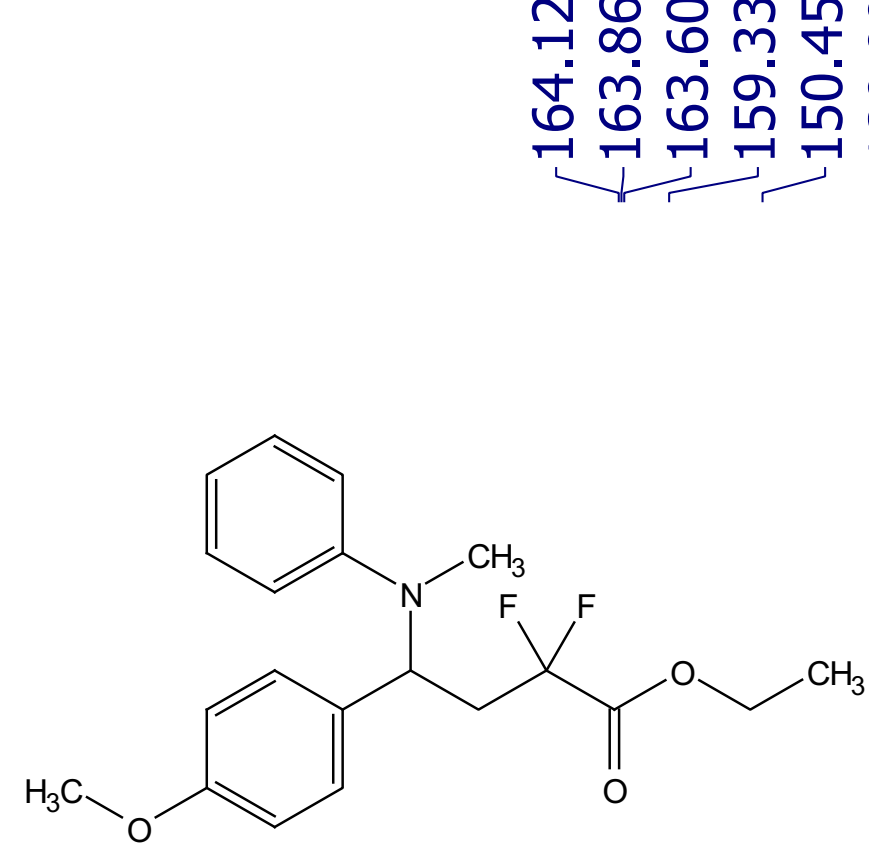

$2 \mathbf{u}$

${ }^{13} \mathrm{C} \mathrm{NMR}, 126 \mathrm{MHz}, \mathrm{CDCl}_{3}$

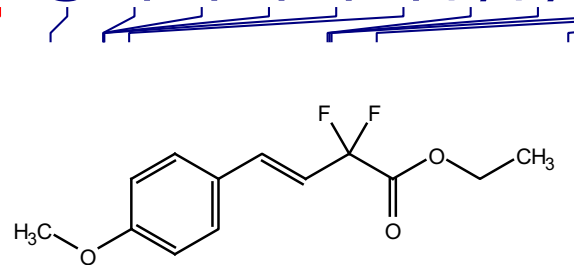

(6\% impurity) 


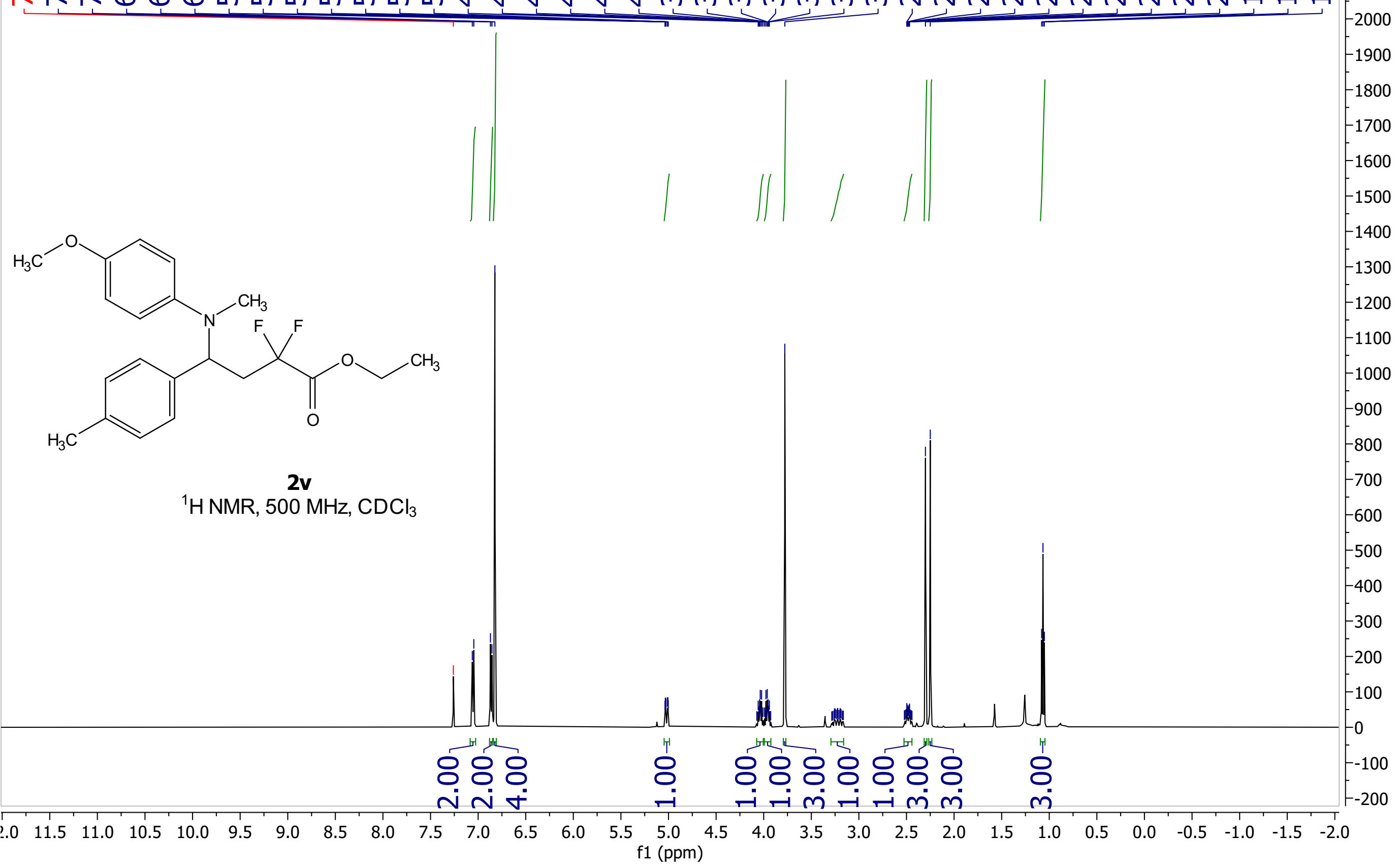




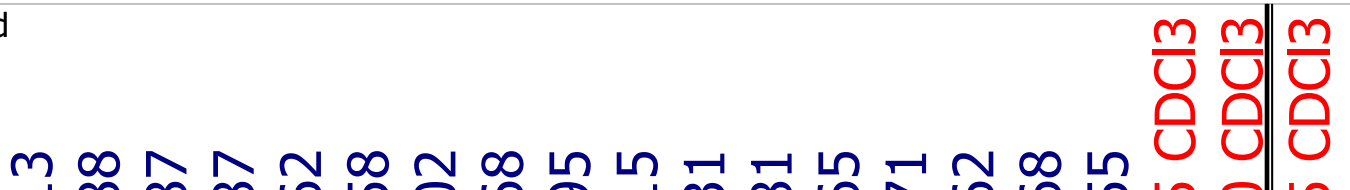

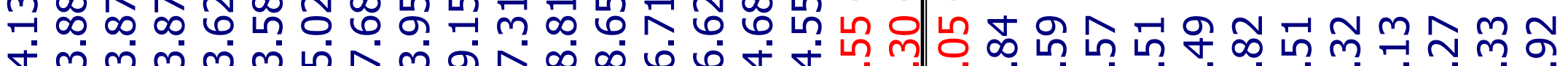
ஏ

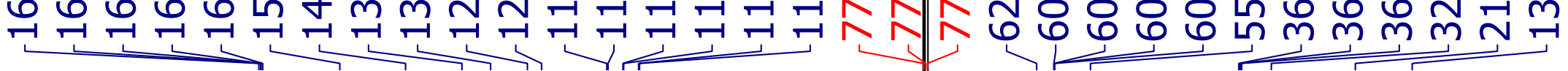

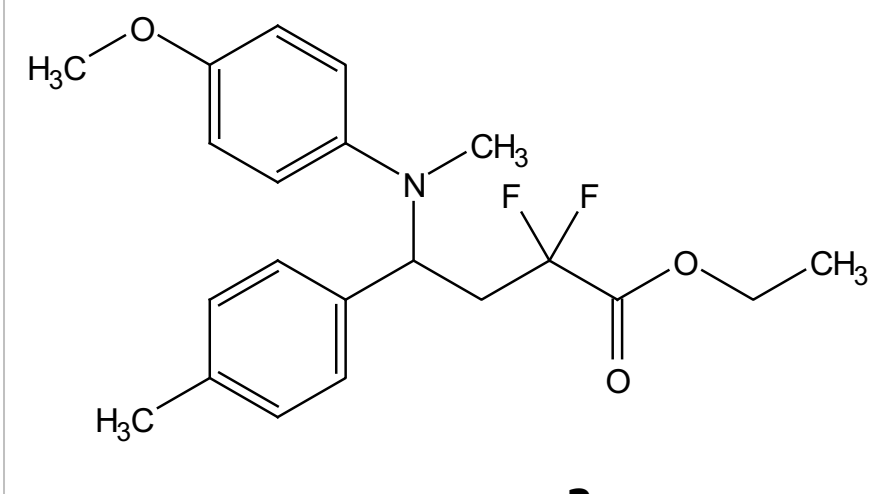

2v

${ }^{13} \mathrm{C} \mathrm{NMR}, 126 \mathrm{MHz}, \mathrm{CDCl}_{3}$

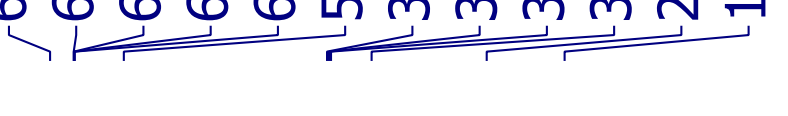




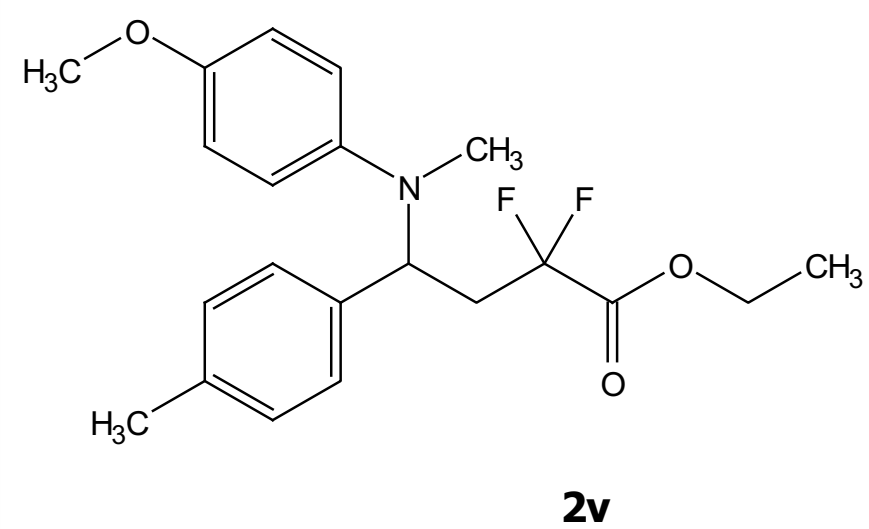

${ }^{19} \mathrm{~F} \mathrm{NMR}, 471 \mathrm{MHz}, \mathrm{CDCl}_{3}$ 


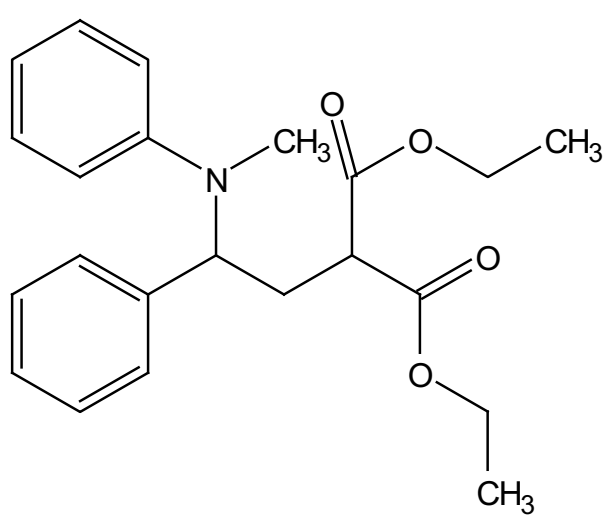

$2 w$

${ }^{1} \mathrm{H} N M R, 500 \mathrm{MHz}, \mathrm{CDCl}_{3}$
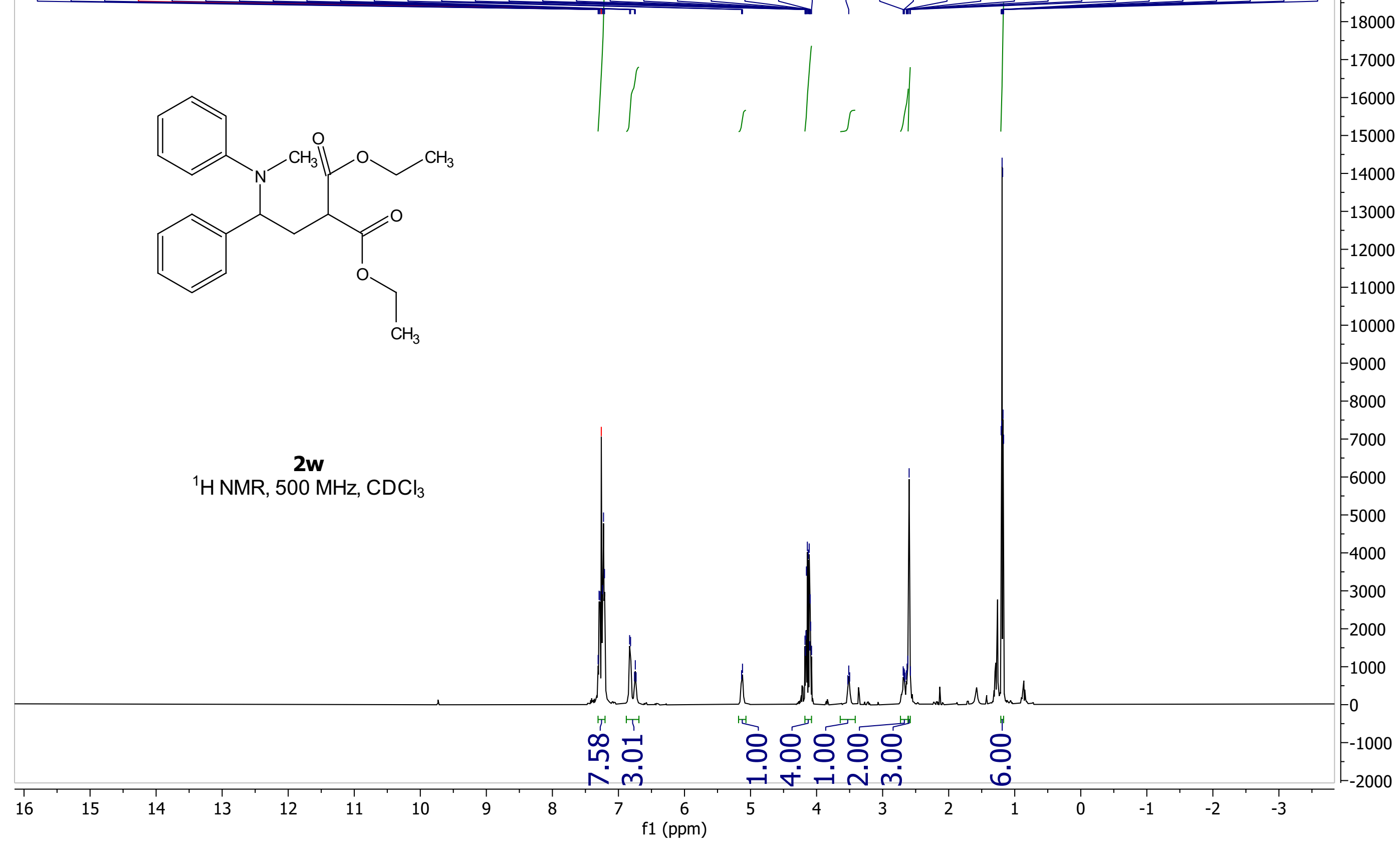
ํㅏㅁำ

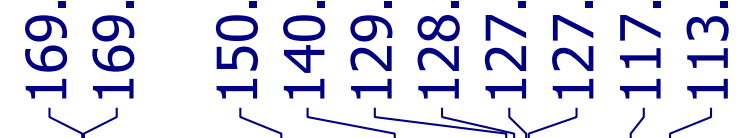

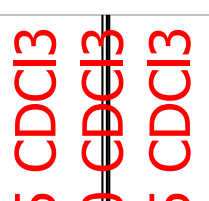

는용ํㅇ 농

$\infty$ ก

^N

№
요

नiं

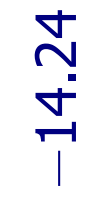

$2 w$

${ }^{13} \mathrm{C} \mathrm{NMR}, 126 \mathrm{MHz}, \mathrm{CDCl}_{3}$ 

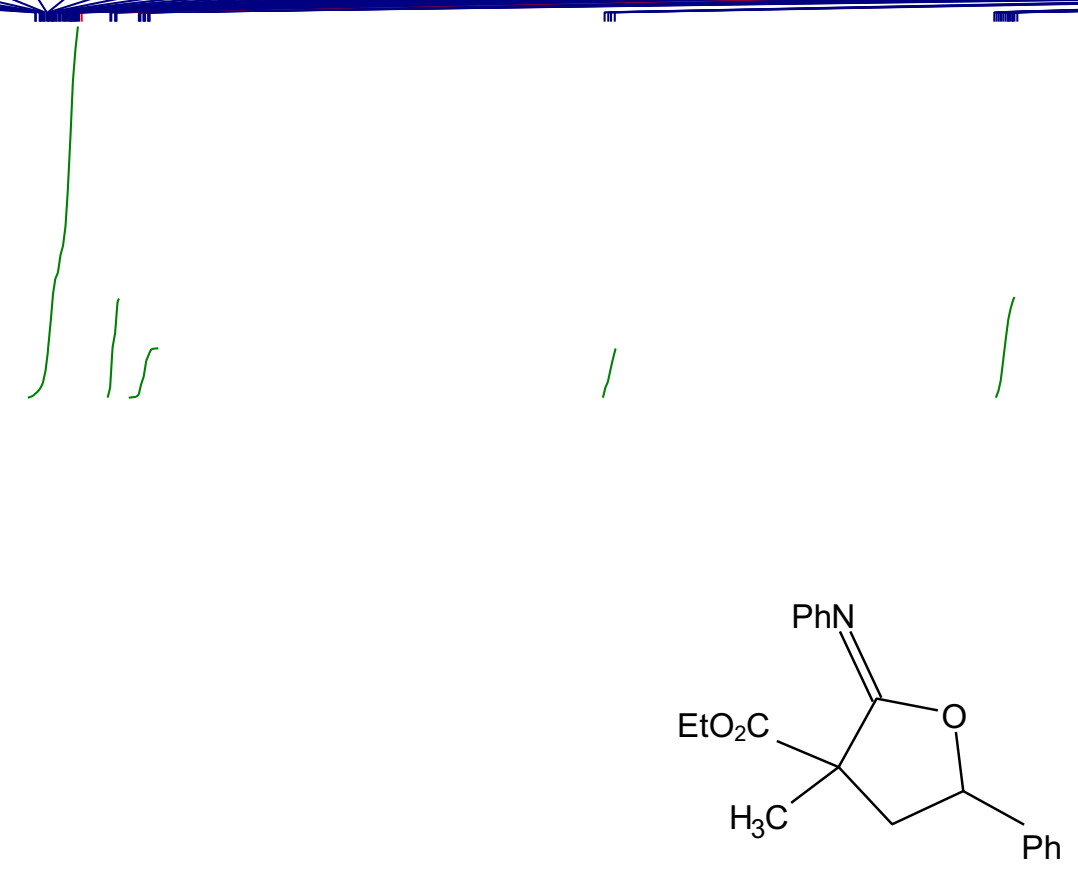

$$
2 x
$$

$1 \mathrm{H} \mathrm{NMR}, 500 \mathrm{MHz}, \mathrm{CDCl} 3$
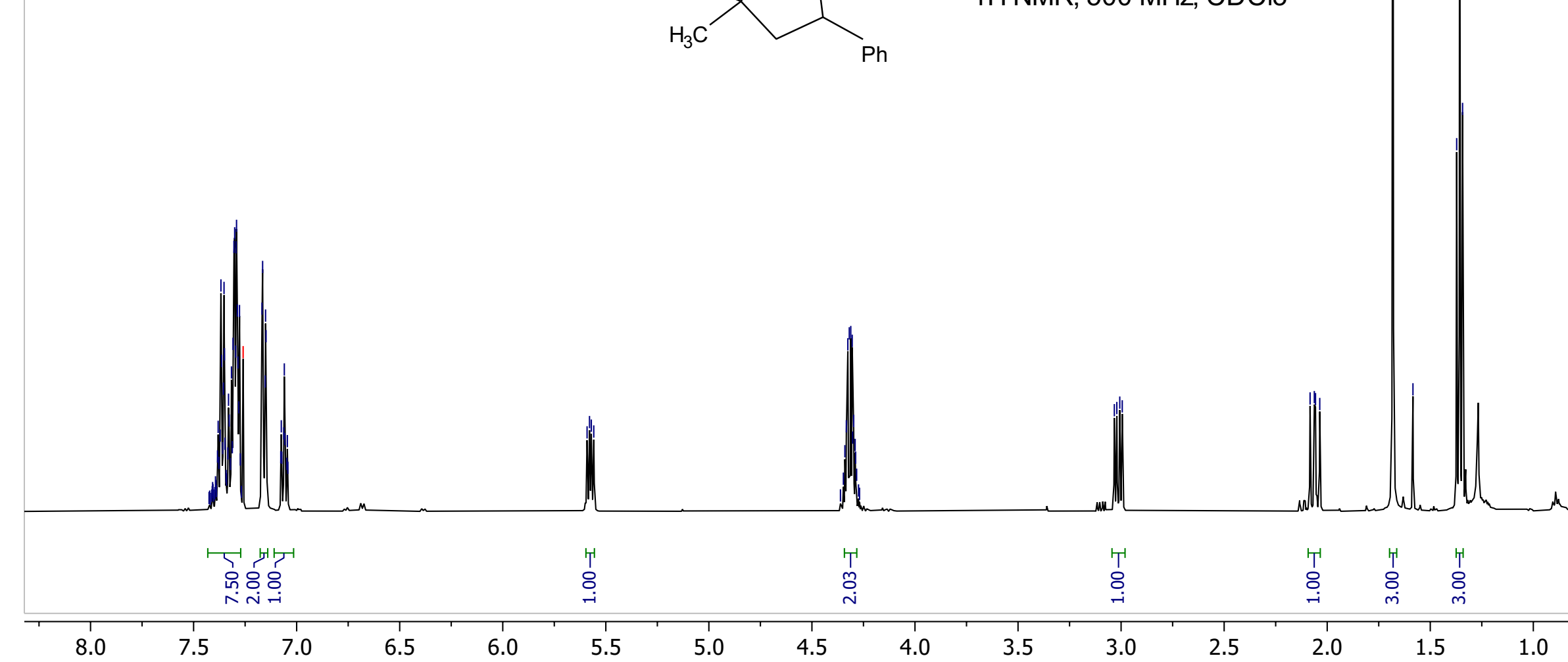

6000

$-4000$

2000 
<smiles>CCOC(=O)C1(C)CC(c2ccccc2)OC1=NO</smiles>

$2 x$

13C NMR, $126 \mathrm{MHz}, \mathrm{CDCl} 3$
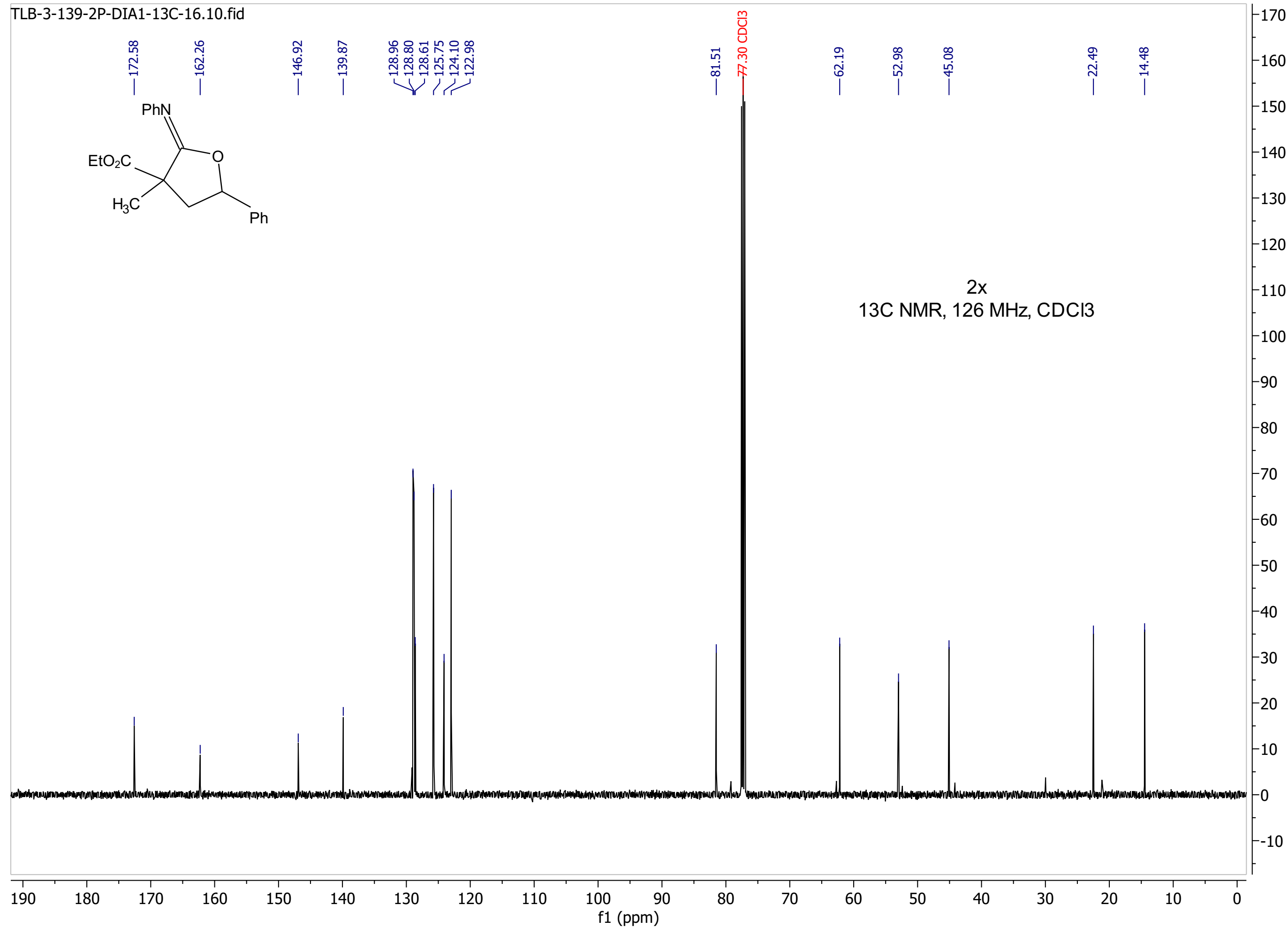


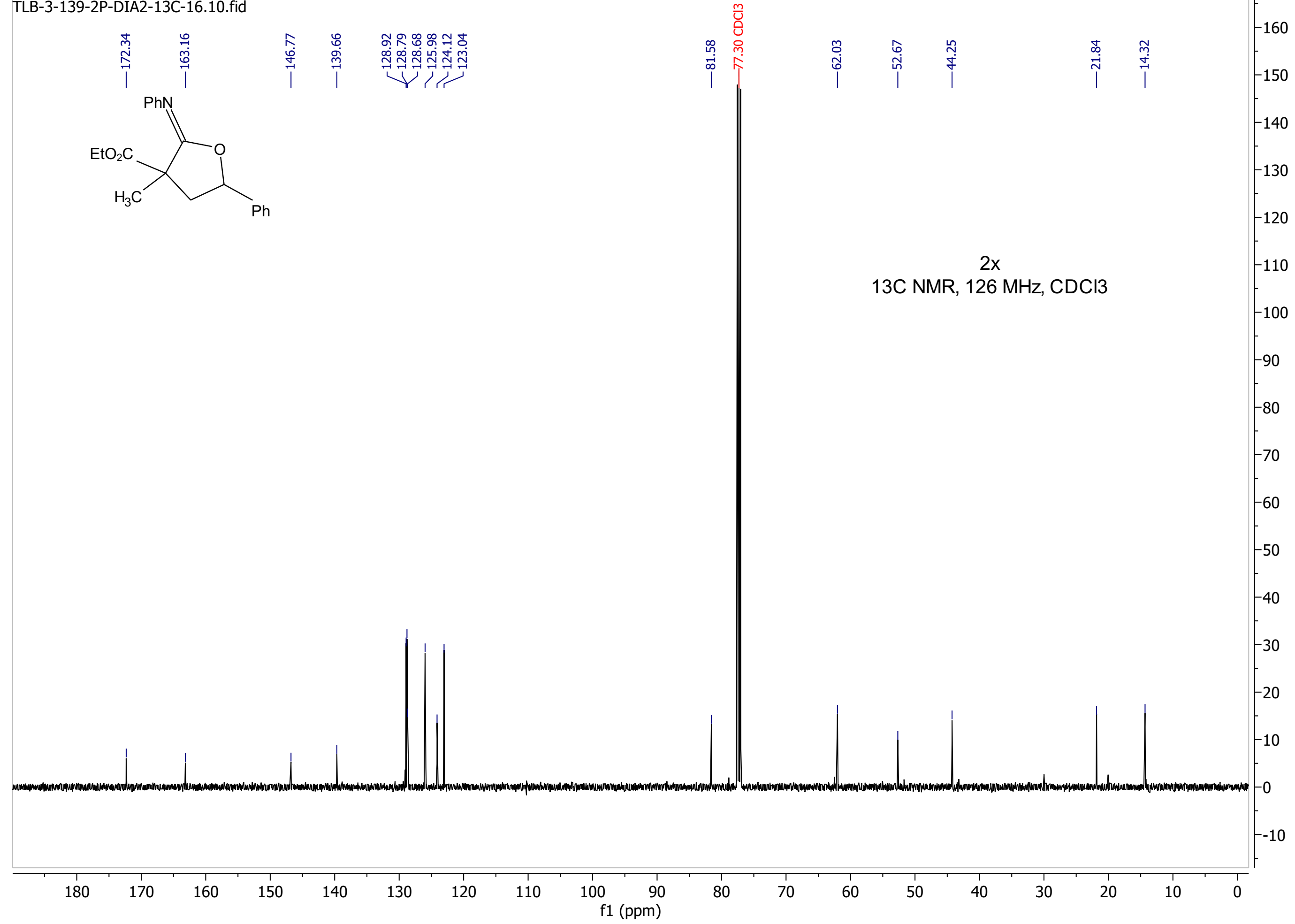




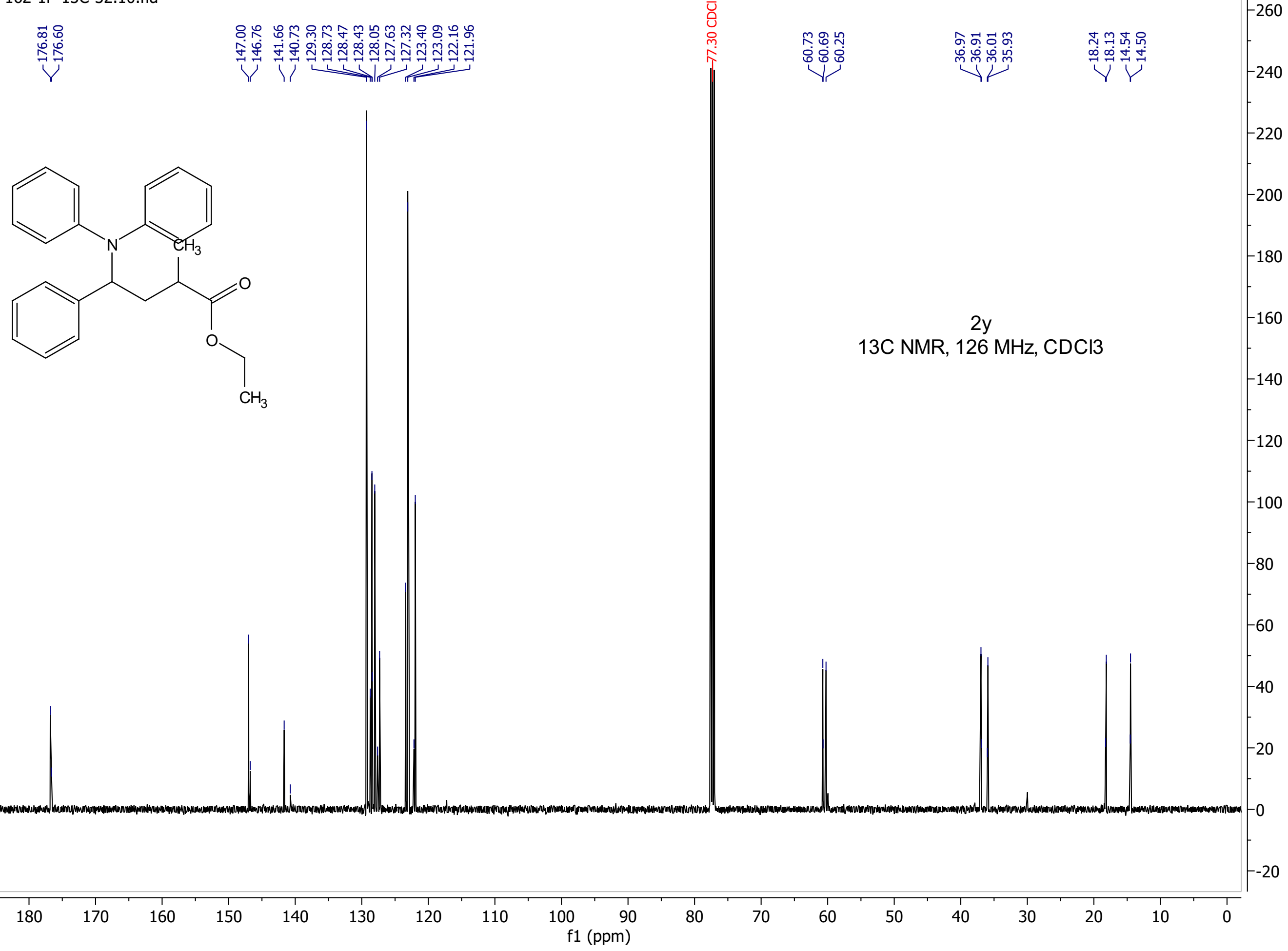




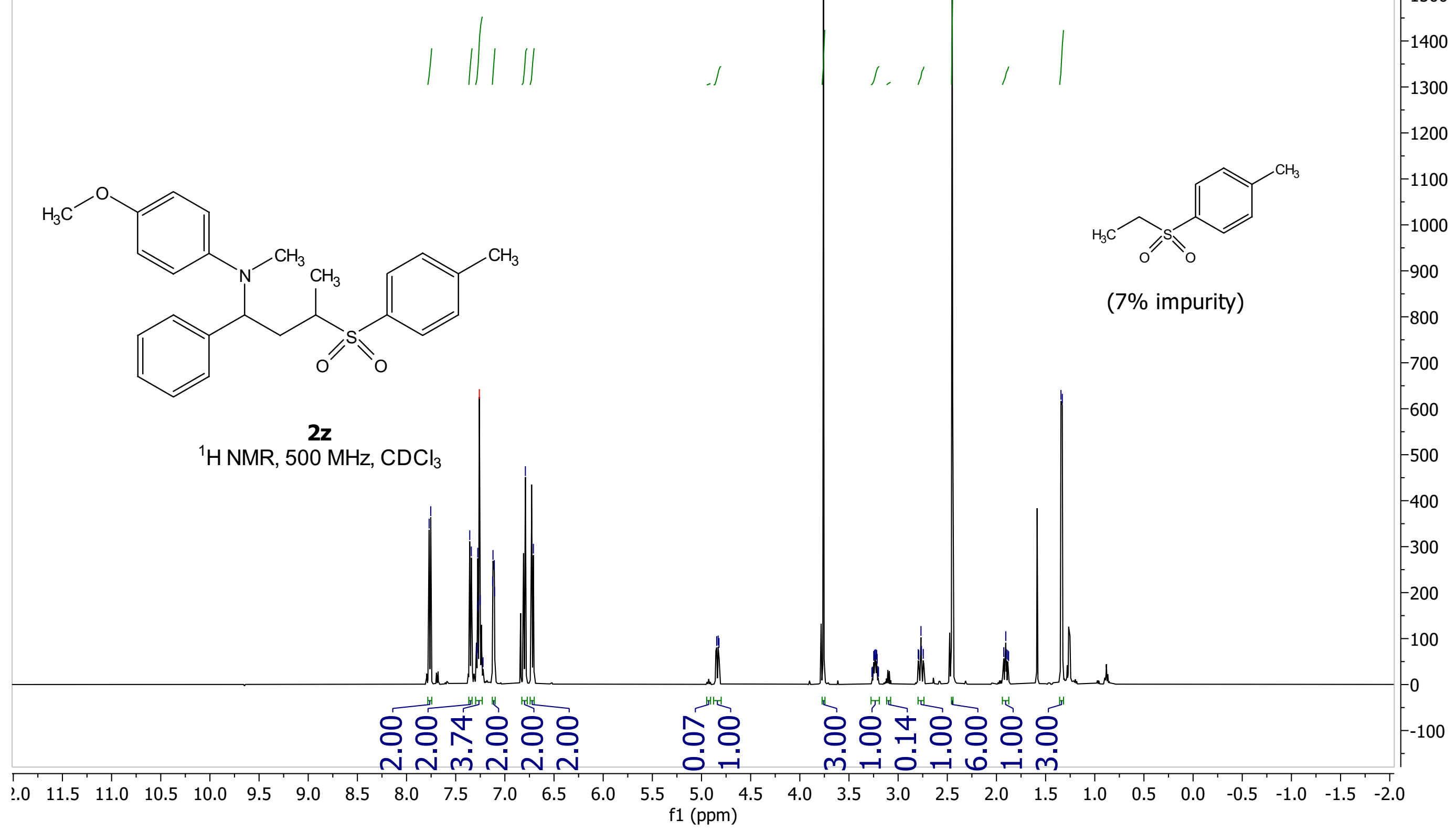




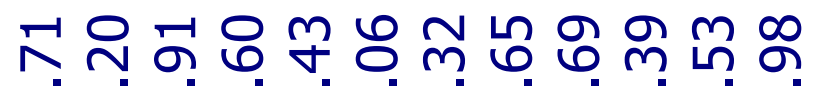

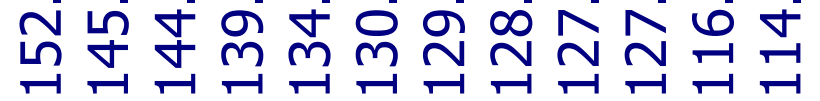

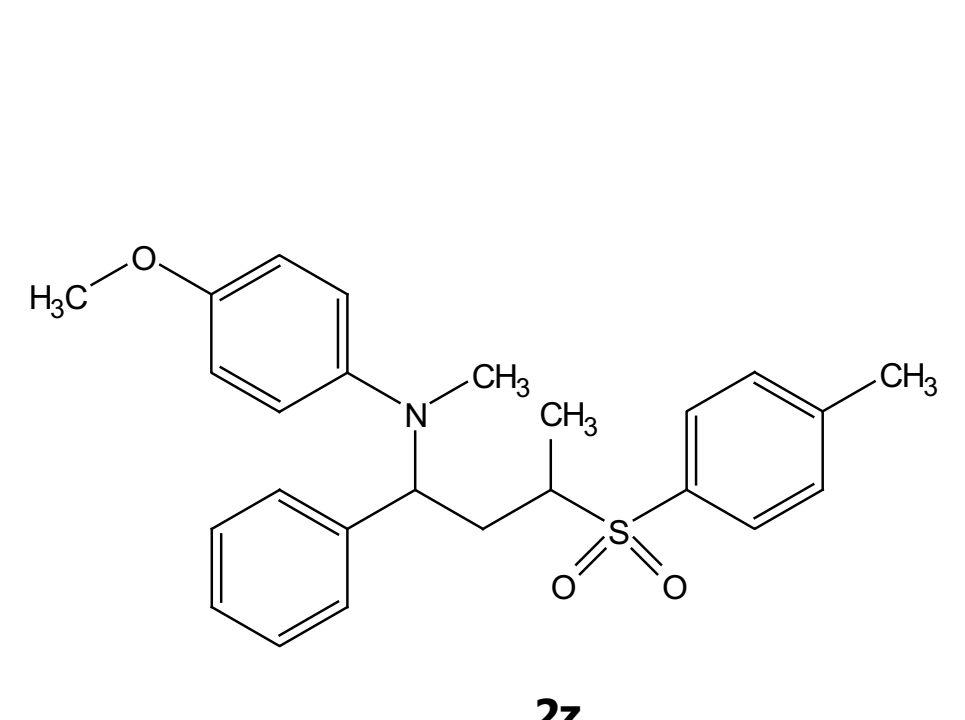

${ }^{13} \mathrm{C} \mathrm{NMR}, 126 \mathrm{MHz}, \mathrm{CDCl}_{3}$

$m$
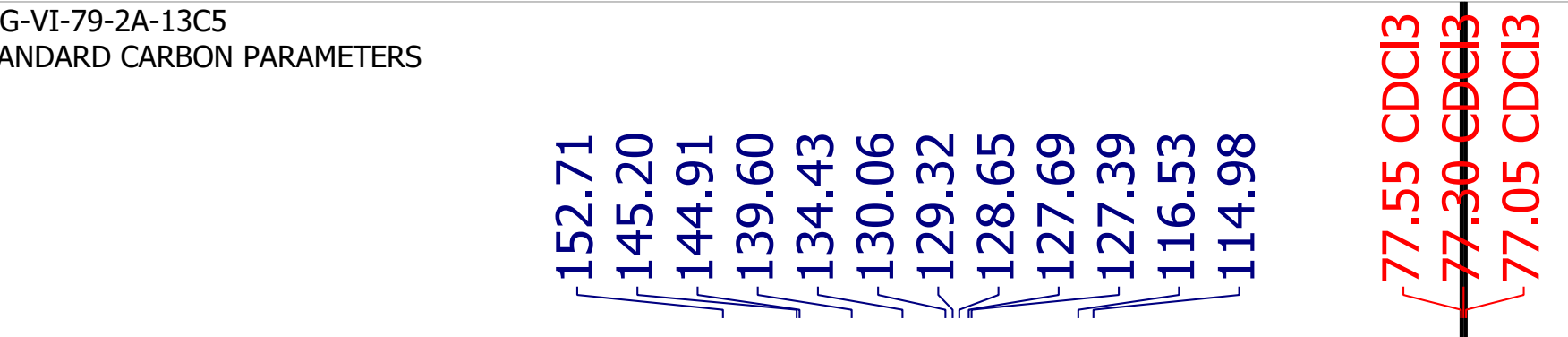

เก็象ํํㅇำ

ヘNㅇํำ

ดำ ํㅗ

mे

$m m \sim$

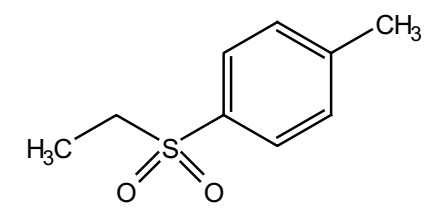

(7\% impurity)

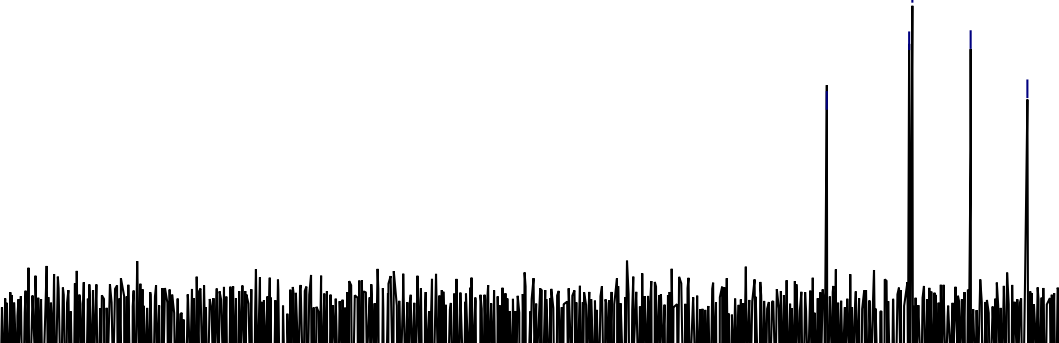




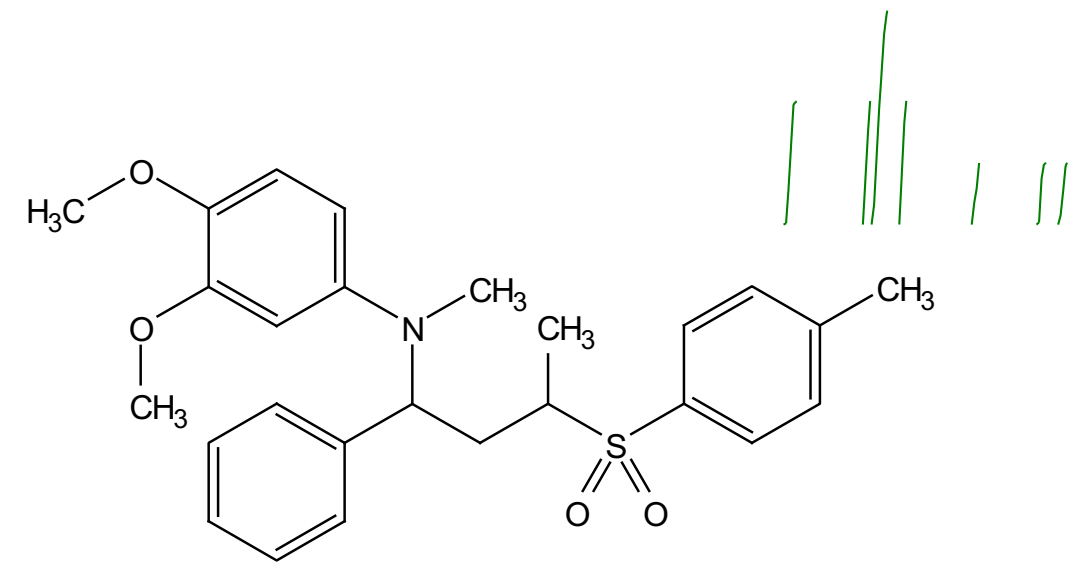

2aa

${ }^{1} \mathrm{H} \mathrm{NMR}, 500 \mathrm{MHz}, \mathrm{CDCl}_{3}$
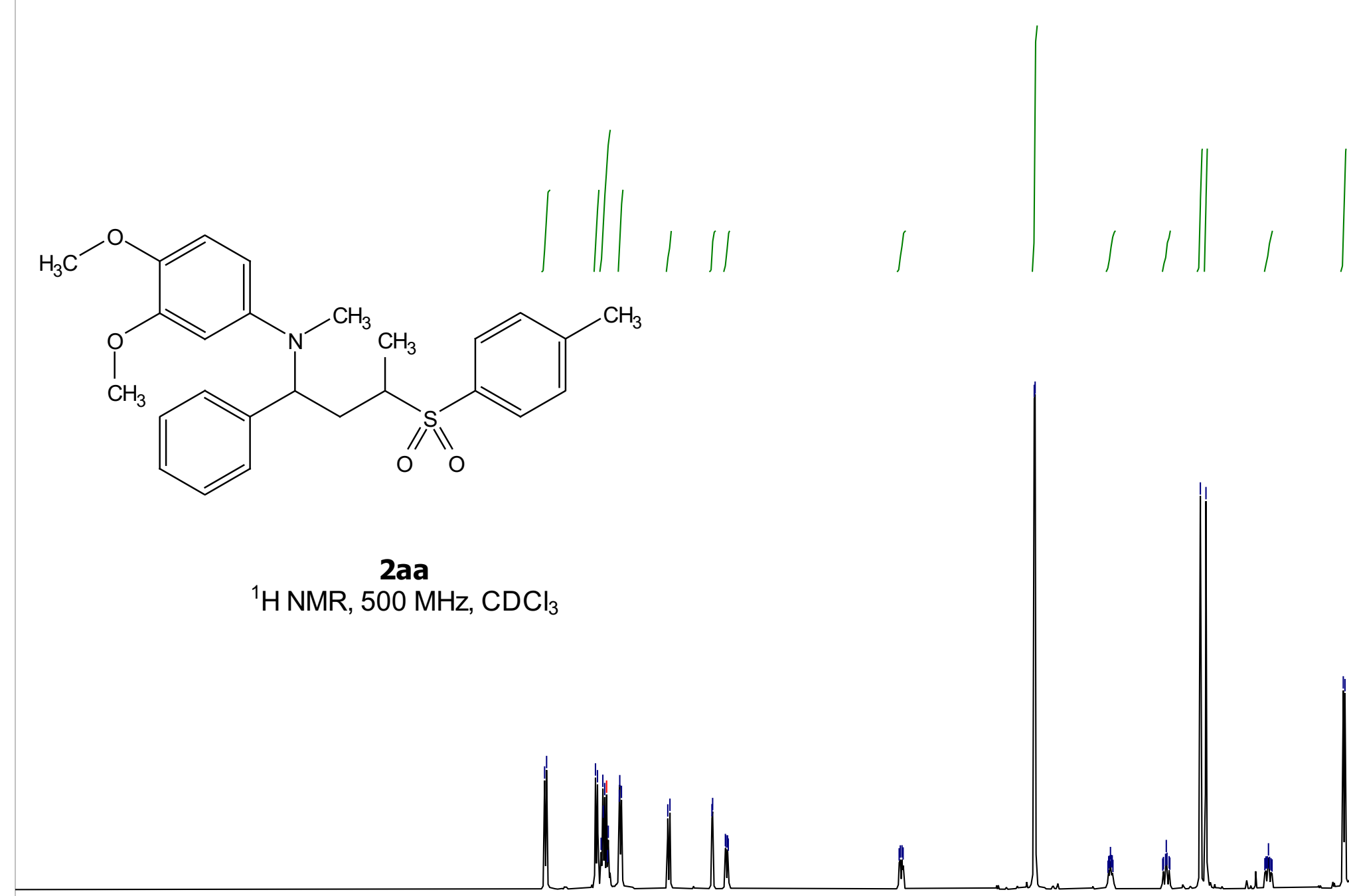


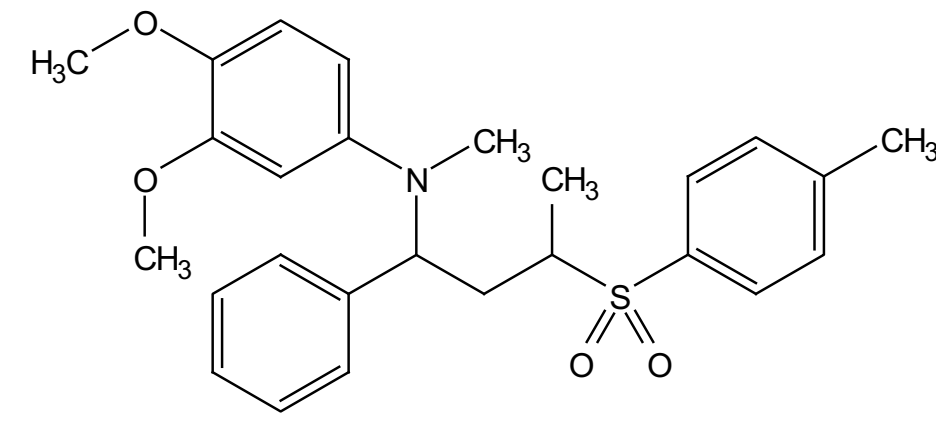

$2 a a$

${ }^{13} \mathrm{C} \mathrm{NMR,}, 126 \mathrm{MHz}, \mathrm{CDCl}_{3}$

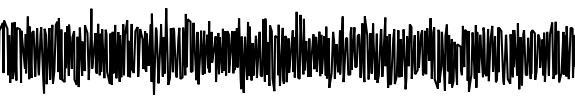

$$
\mathrm{MHz}_{3}
$$




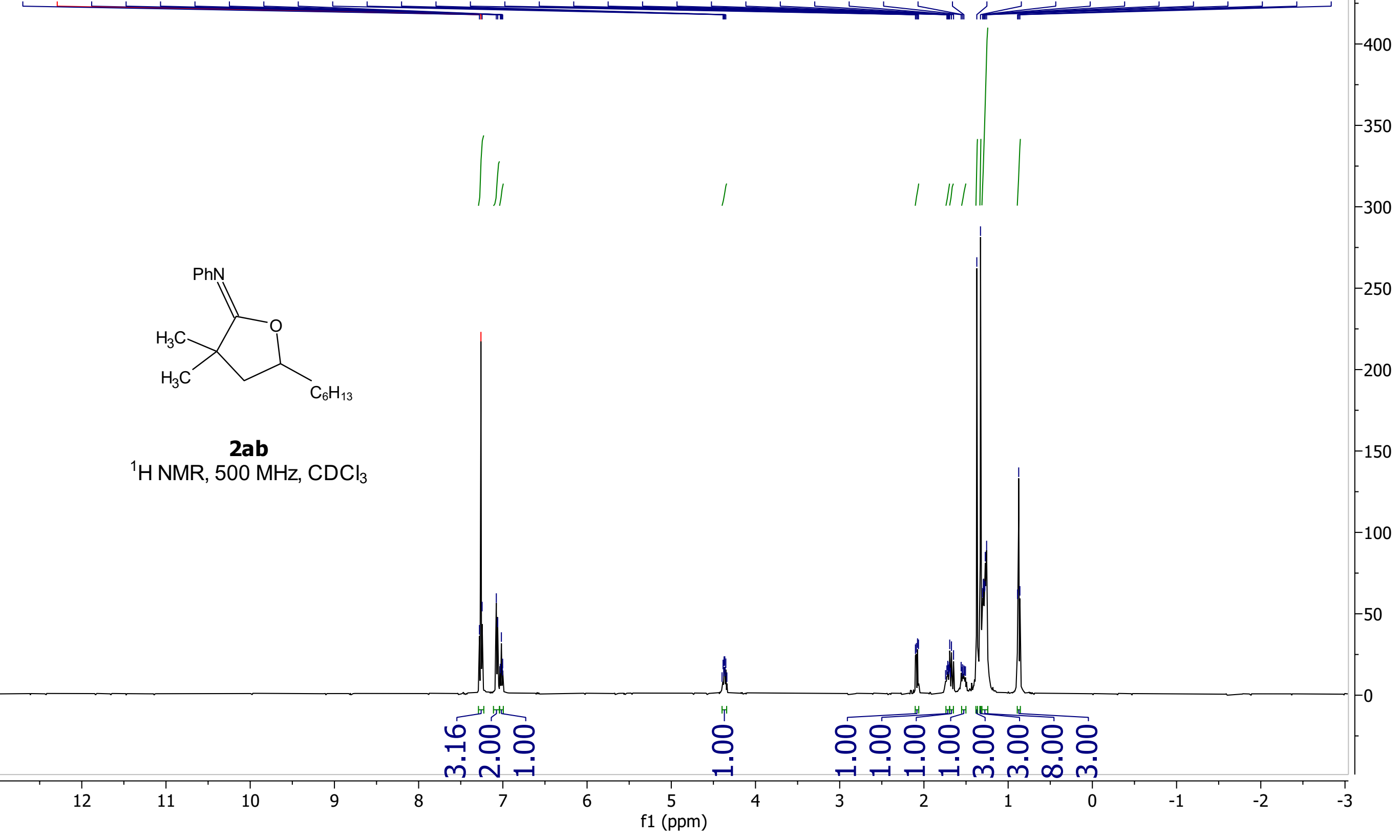




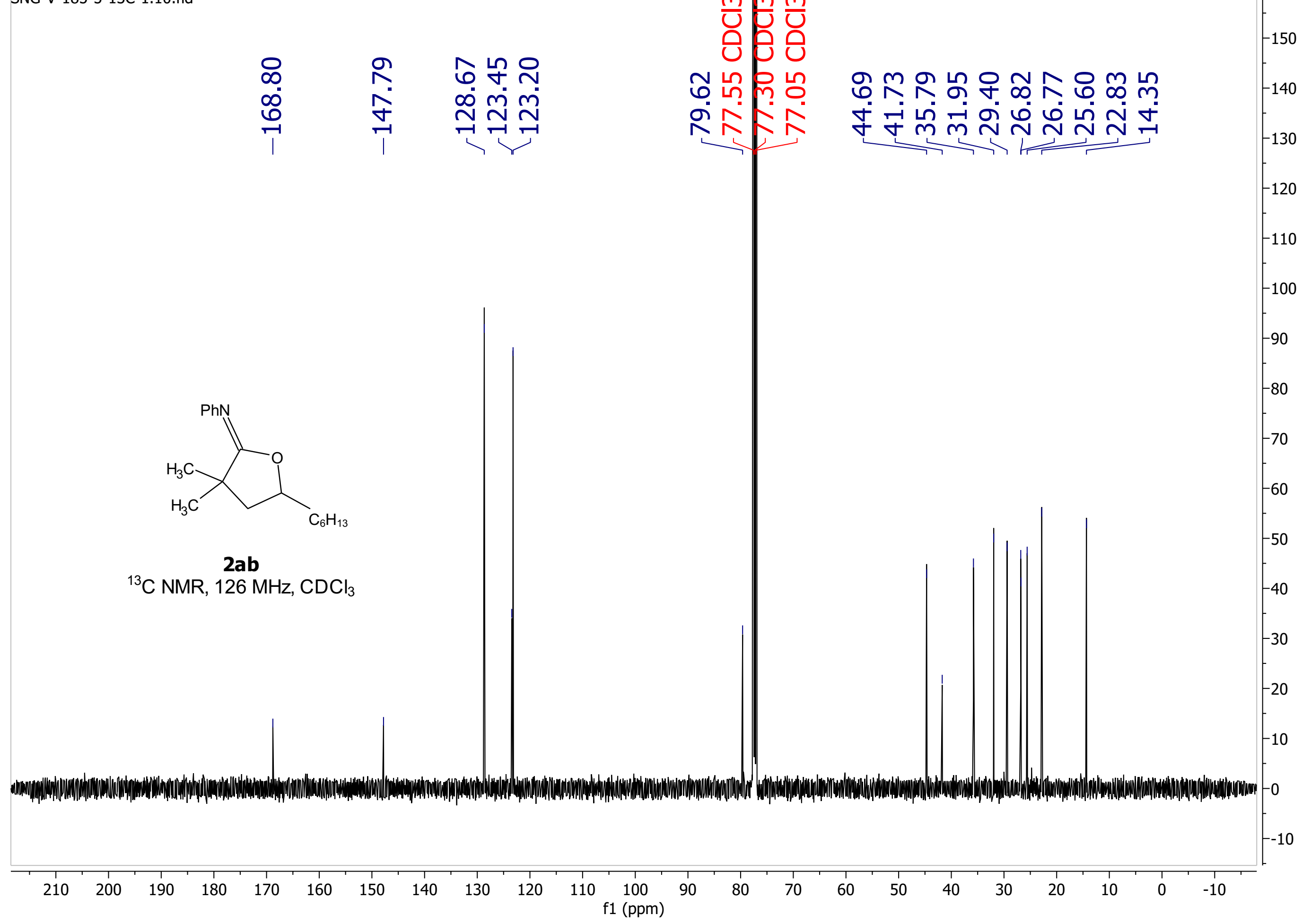




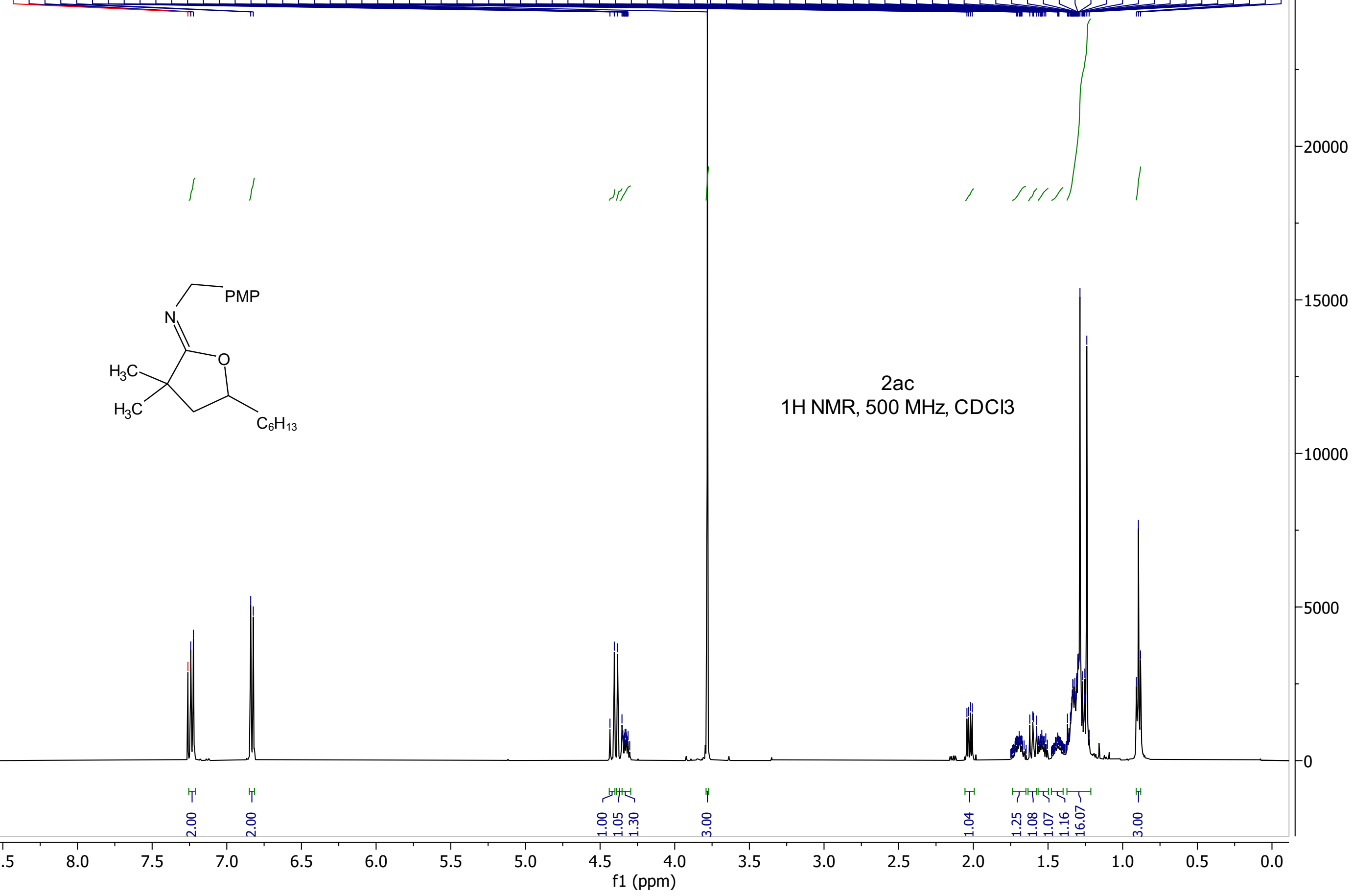




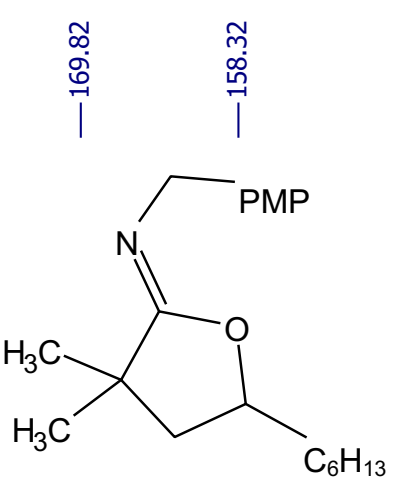

$2 \mathrm{ac}$

13C NMR, $126 \mathrm{MHz}, \mathrm{CDCl} 3$

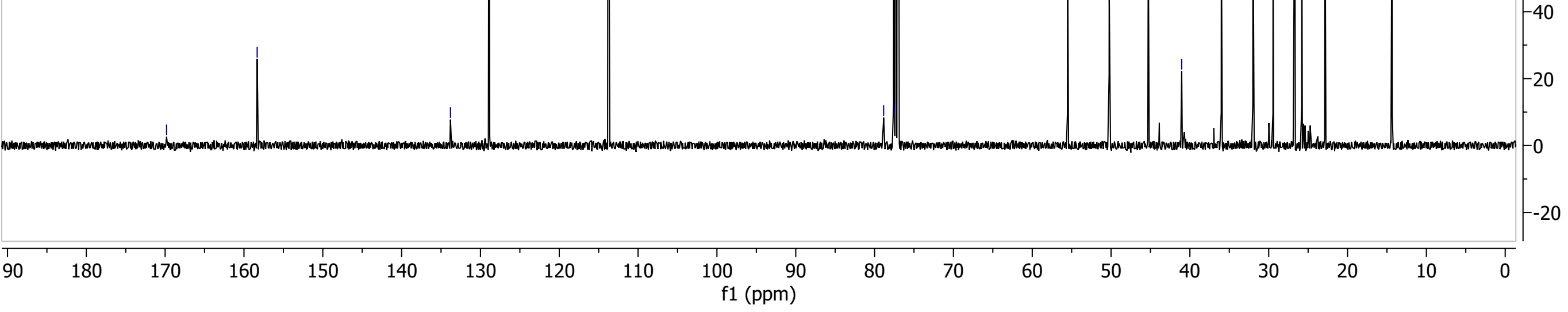




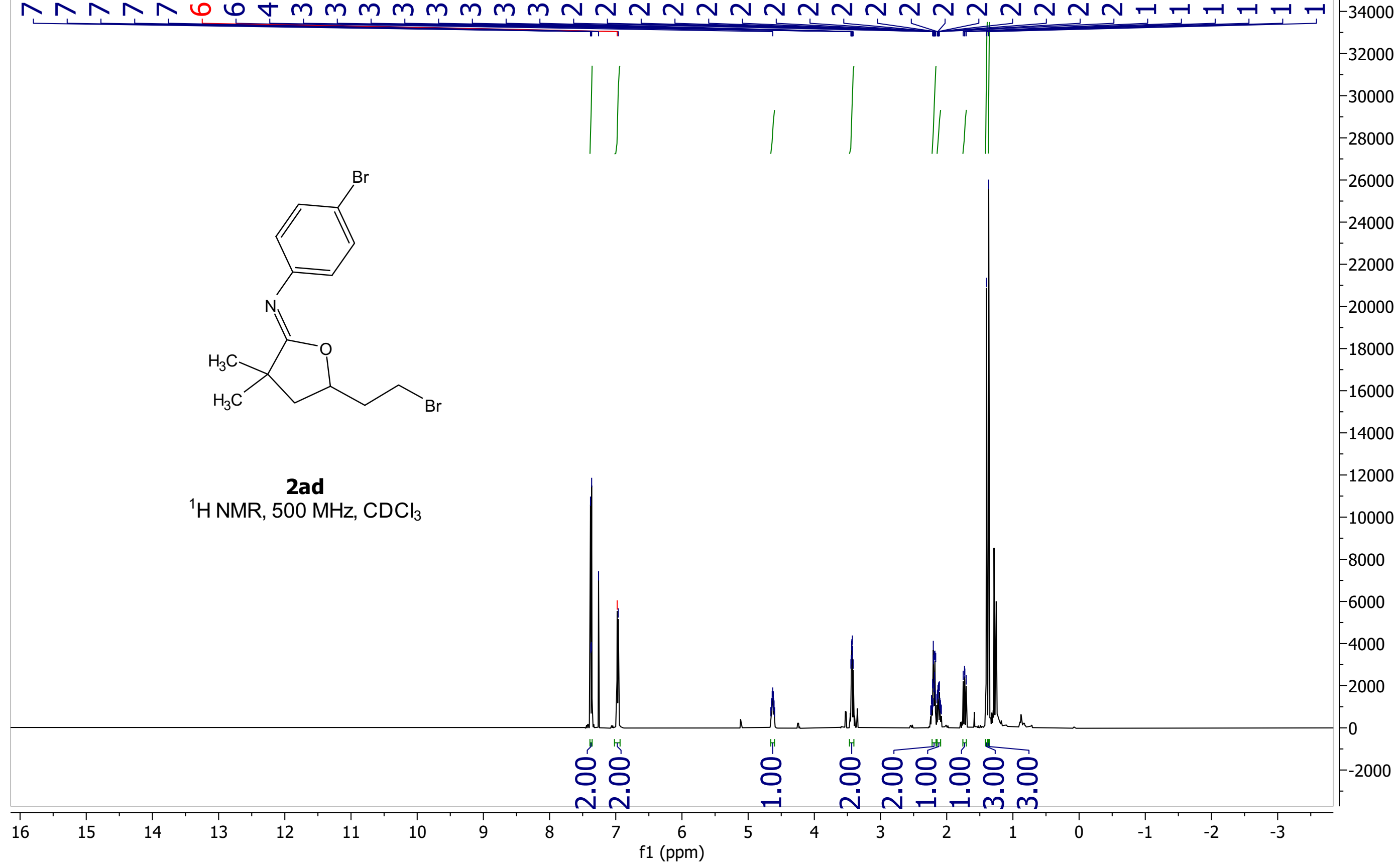




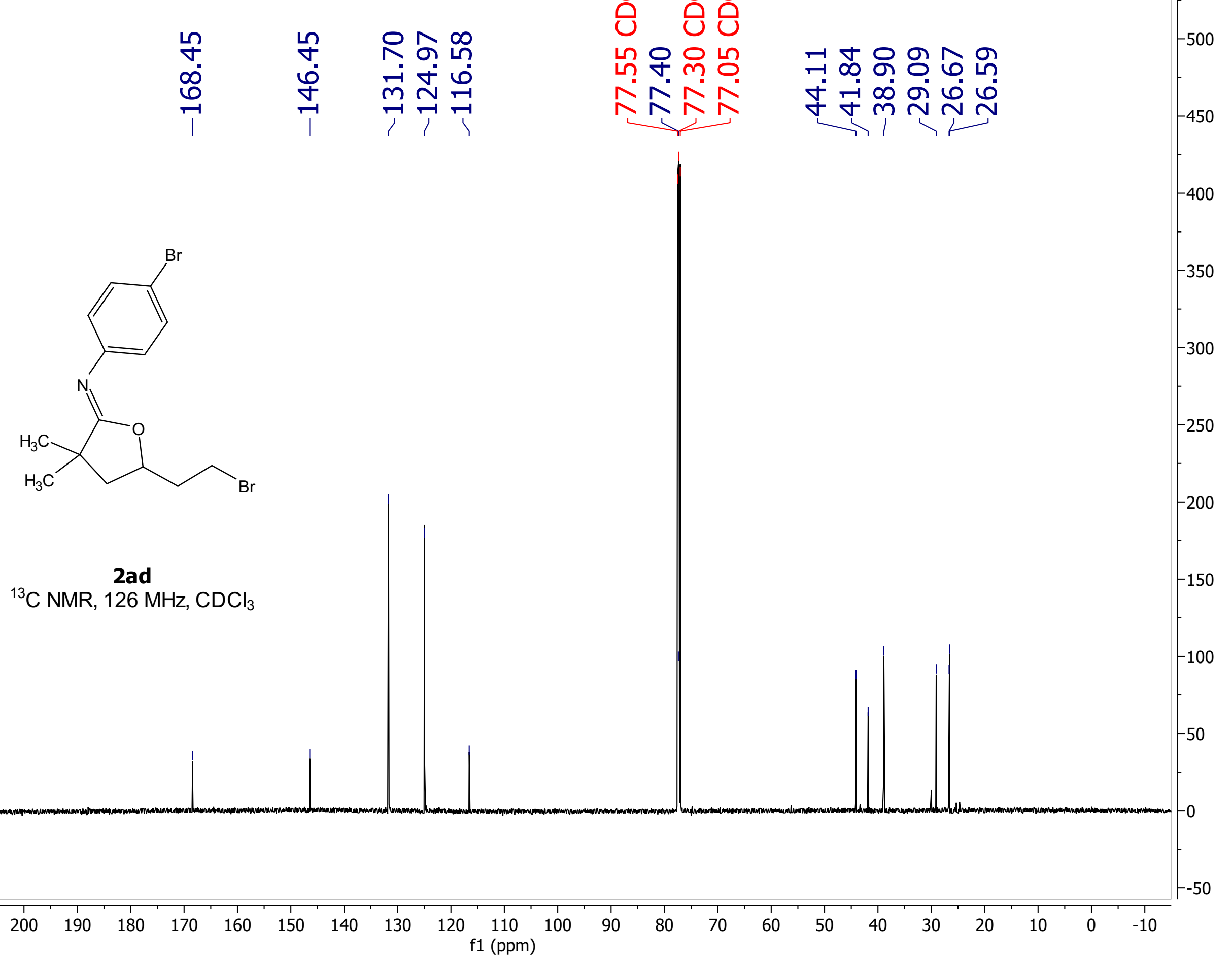



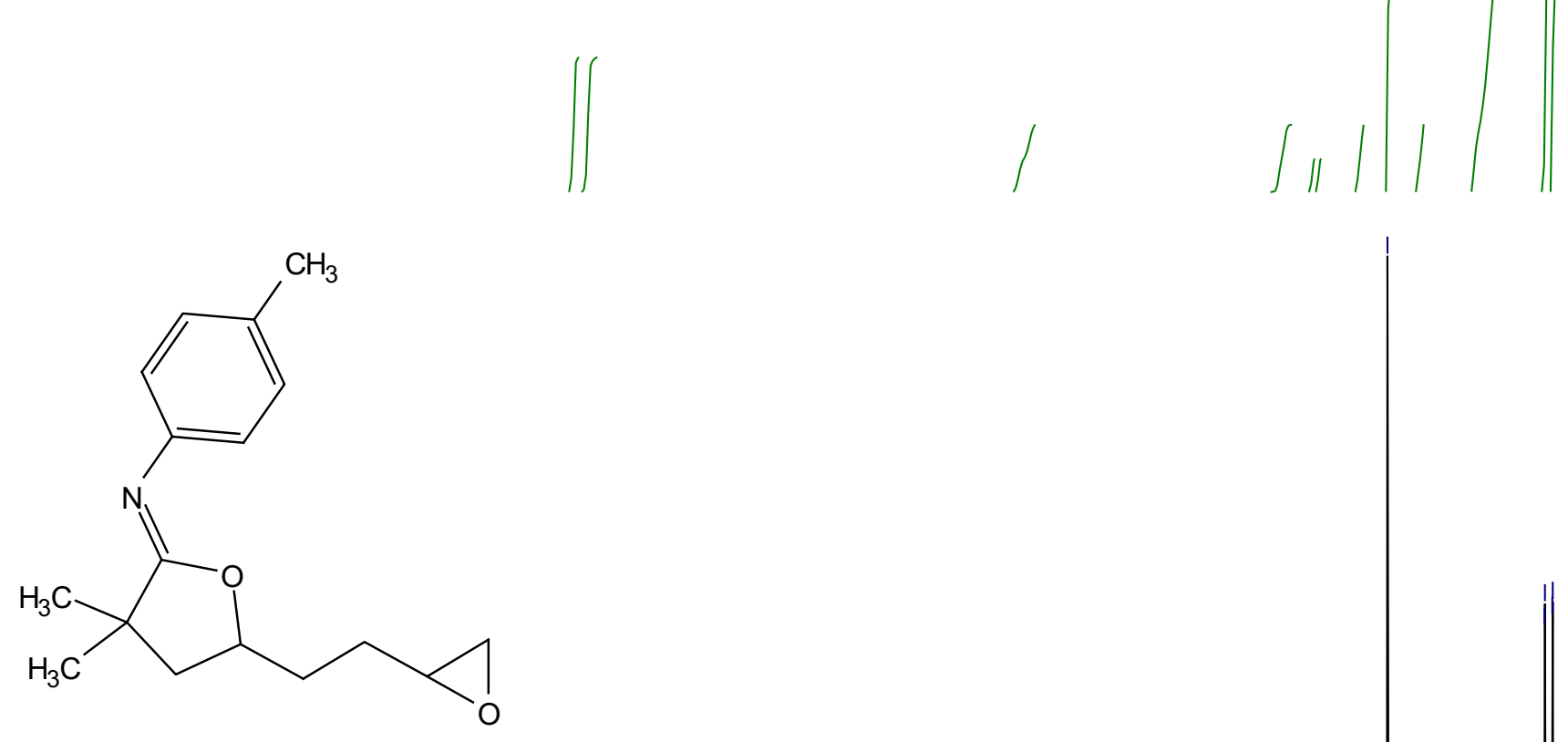

2ae

${ }^{1} \mathrm{H}$ NMR, $500 \mathrm{MHz}, \mathrm{CDCl}_{3}$

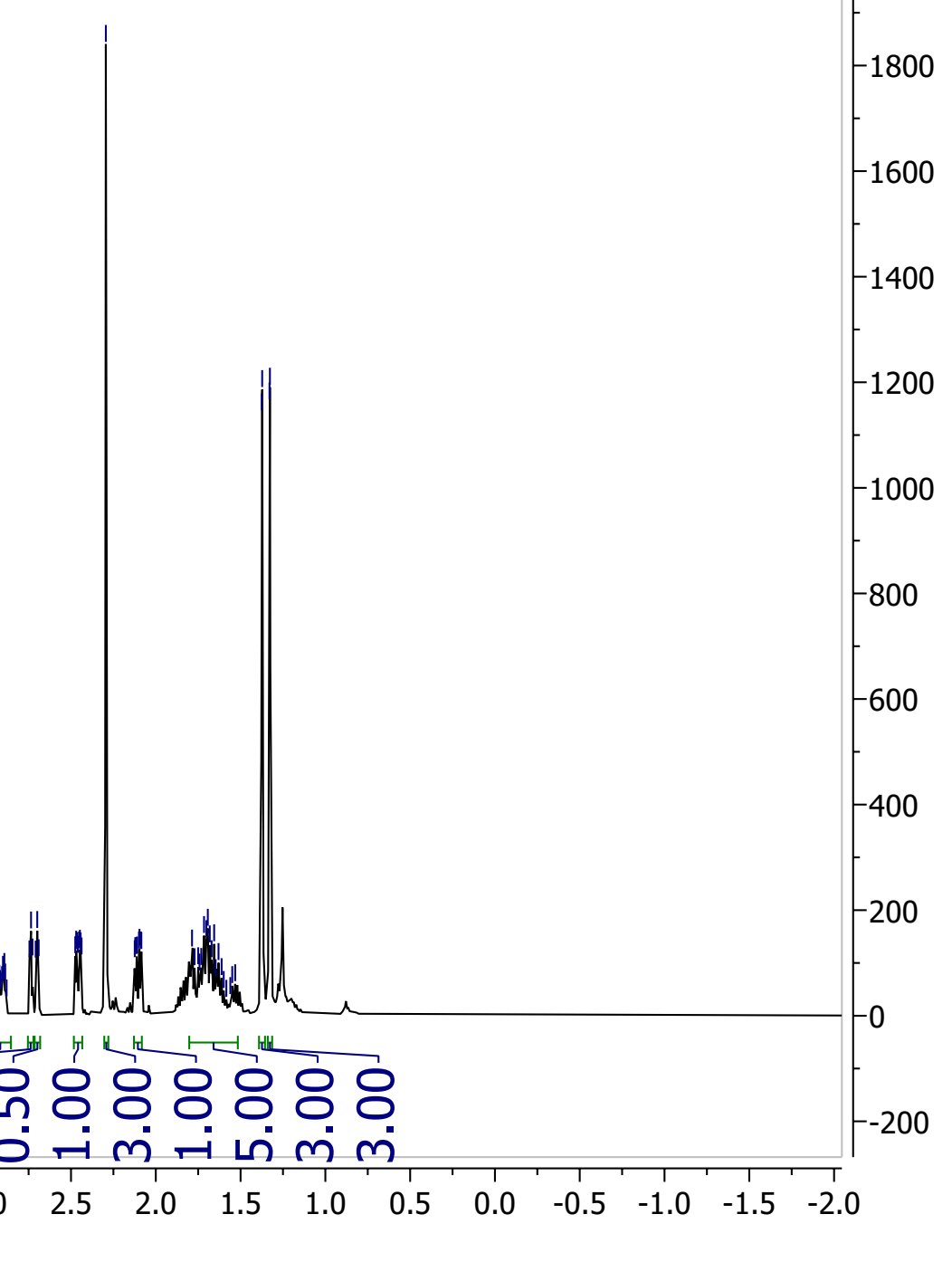




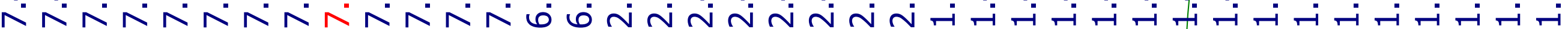

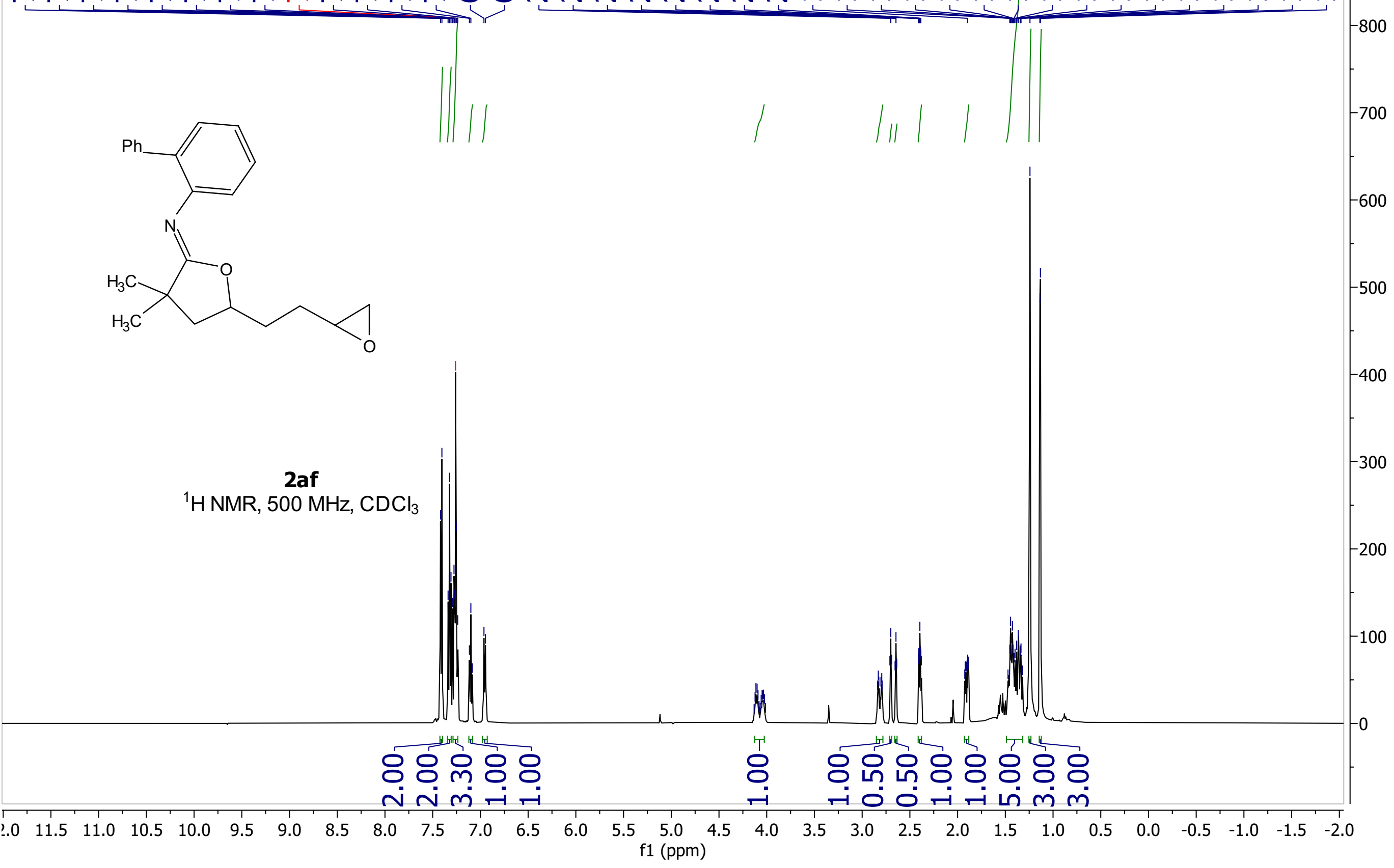




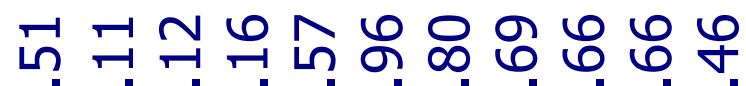


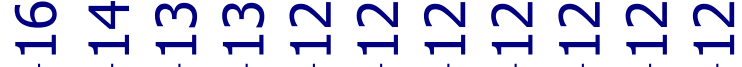
ᄋ $\infty \infty N$

유 ํㅝ

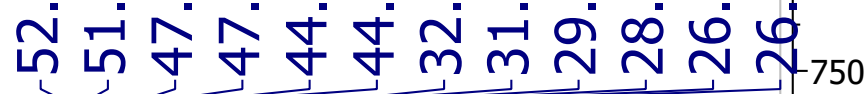

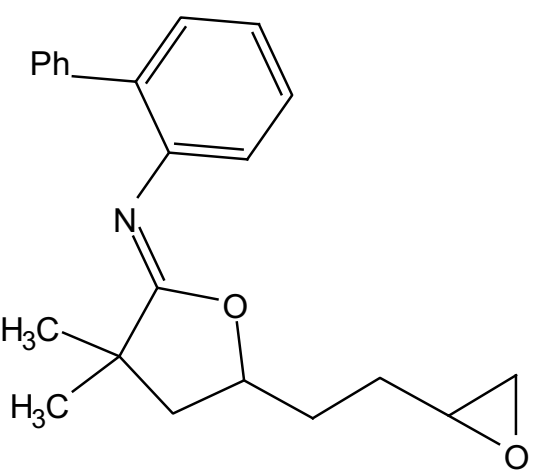

2af

${ }^{13} \mathrm{C} \mathrm{NMR}, 126 \mathrm{MHz}, \mathrm{CDCl}_{3}$
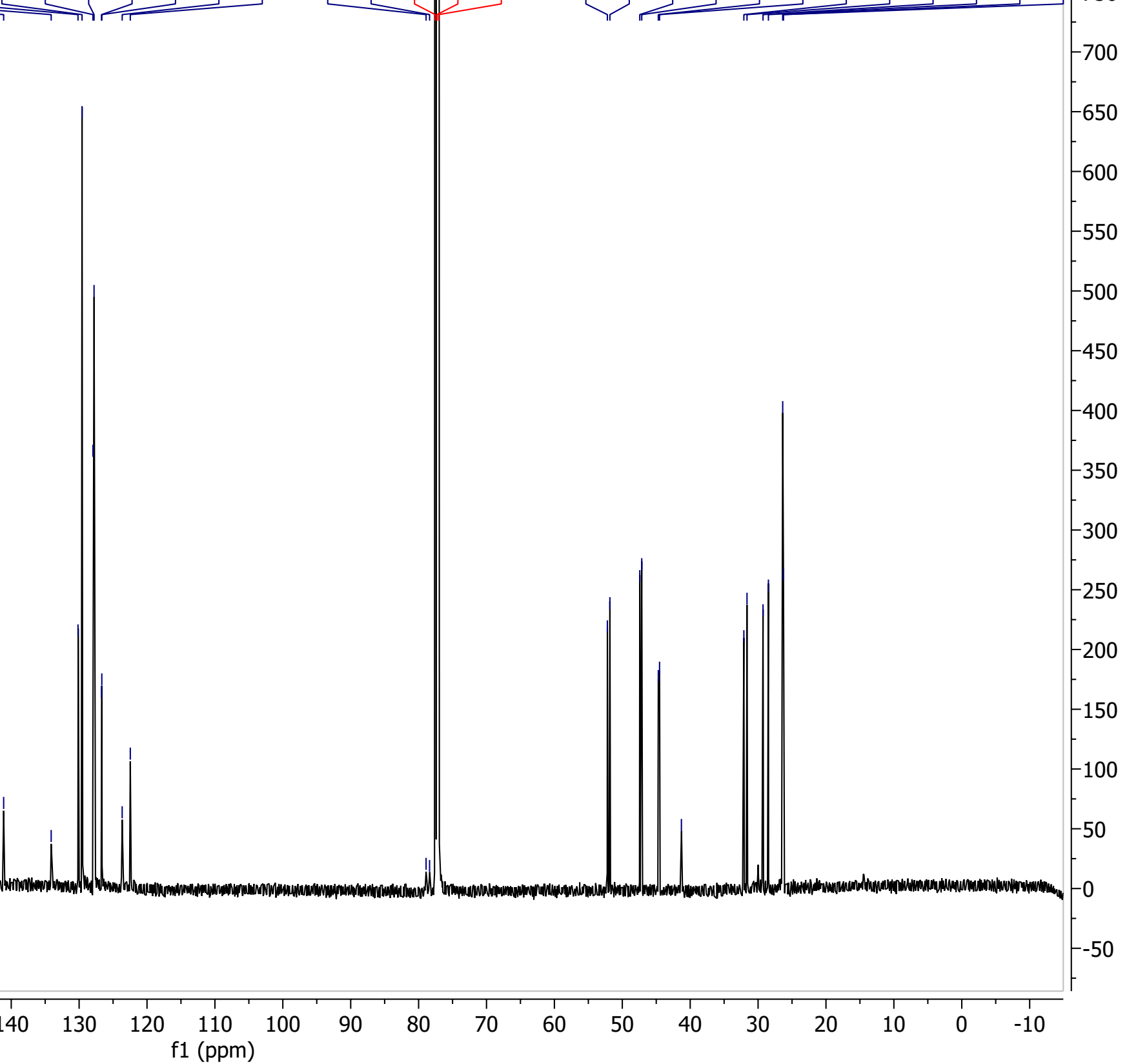


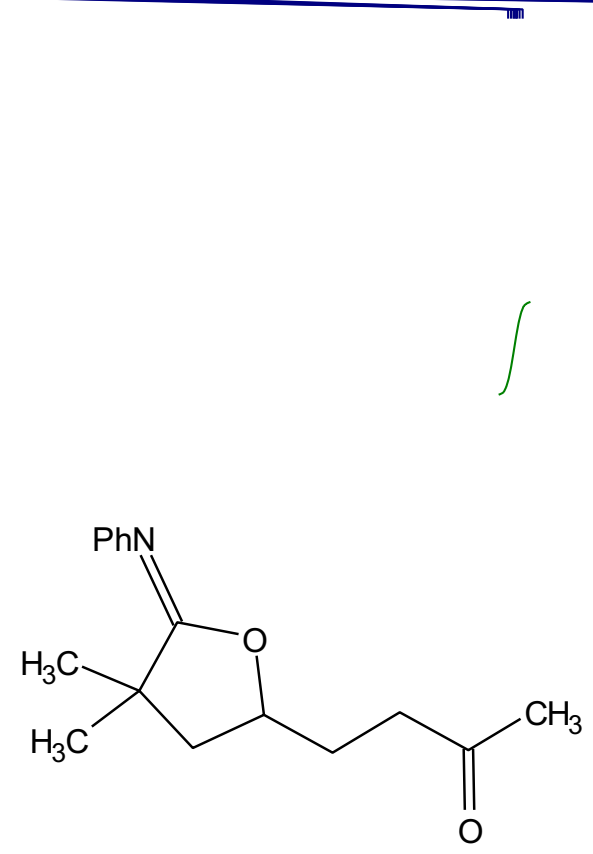

2 ag

$1 \mathrm{H} \mathrm{NMR}, 500 \mathrm{MHz}, \mathrm{CDCl} 3$

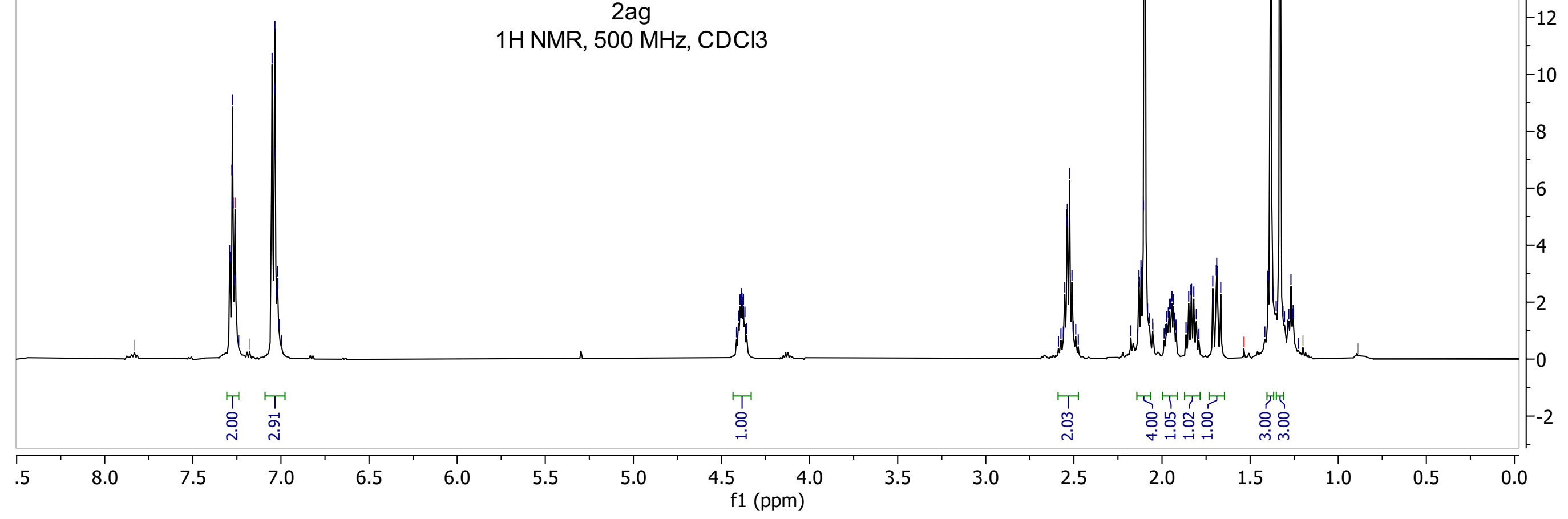



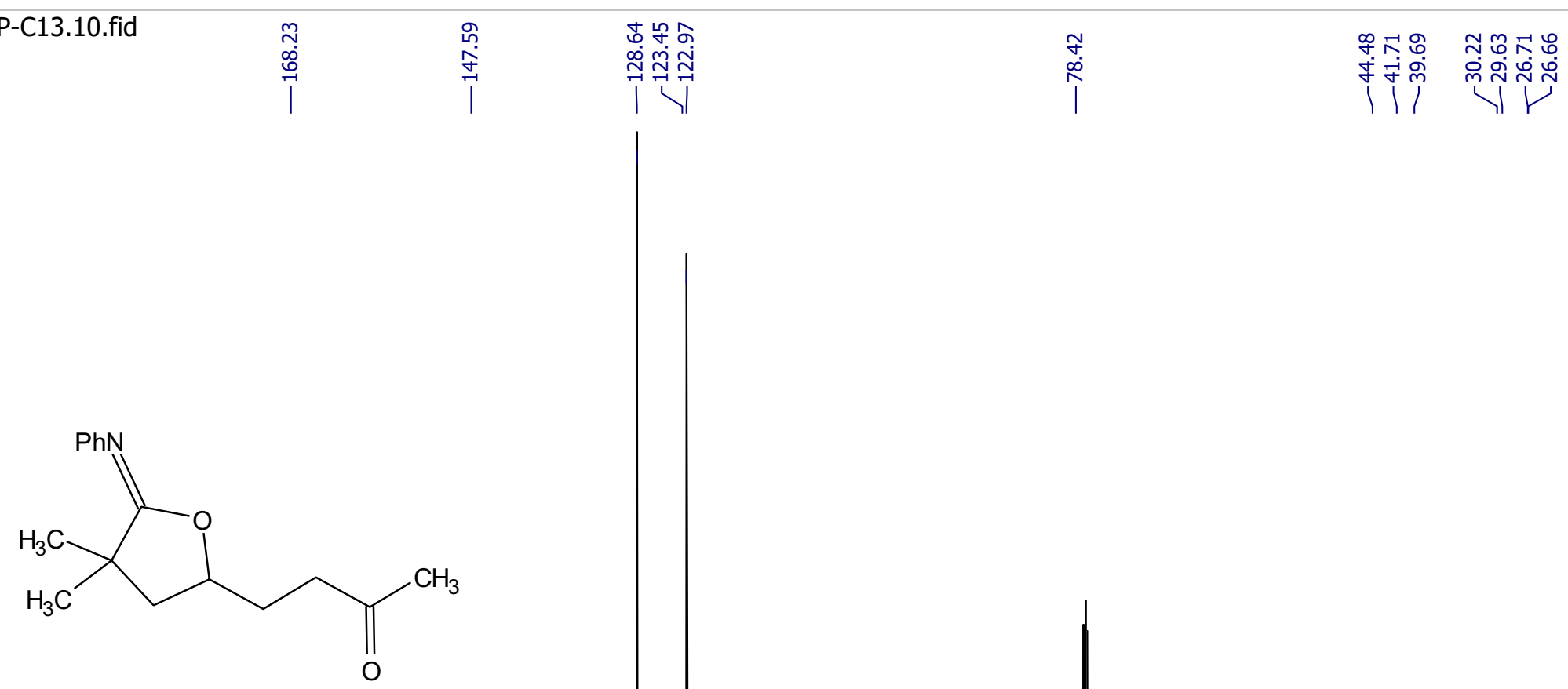

$2 \mathrm{ag}$

1H NMR, $126 \mathrm{MHz}, \mathrm{CDCl} 3$

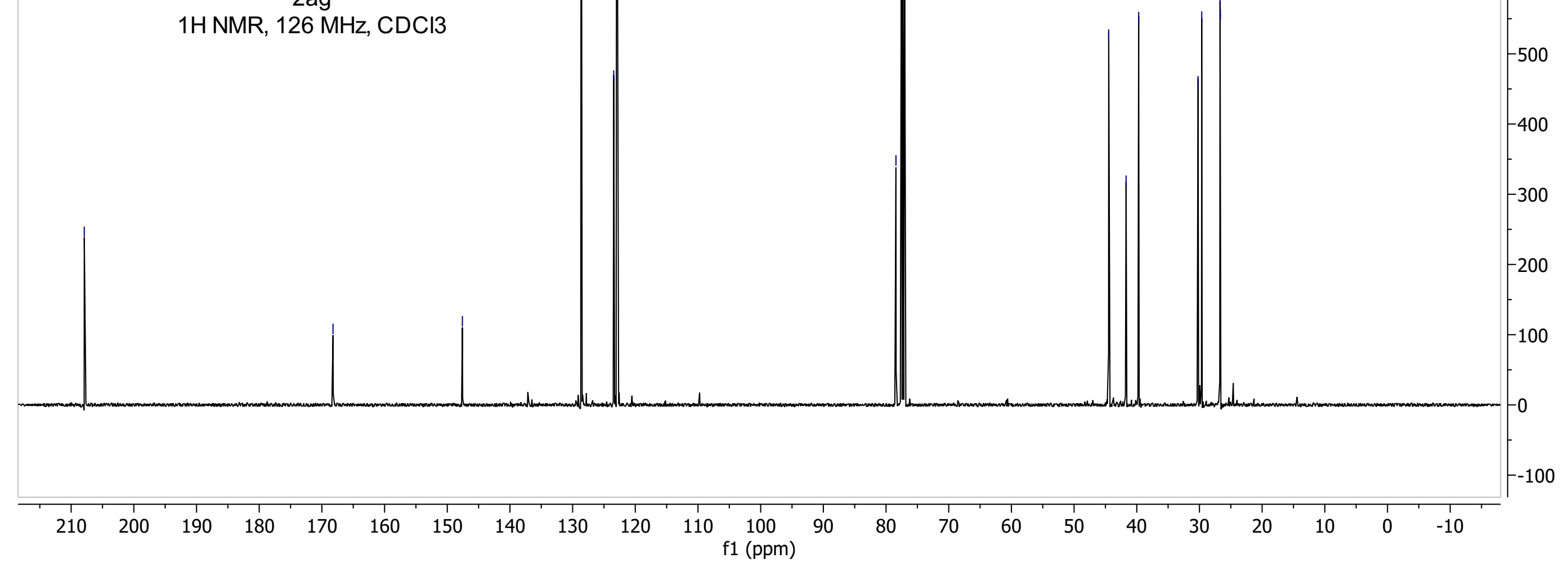


SNG-VI-14-6-4I N

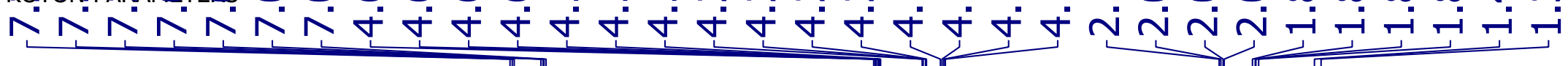
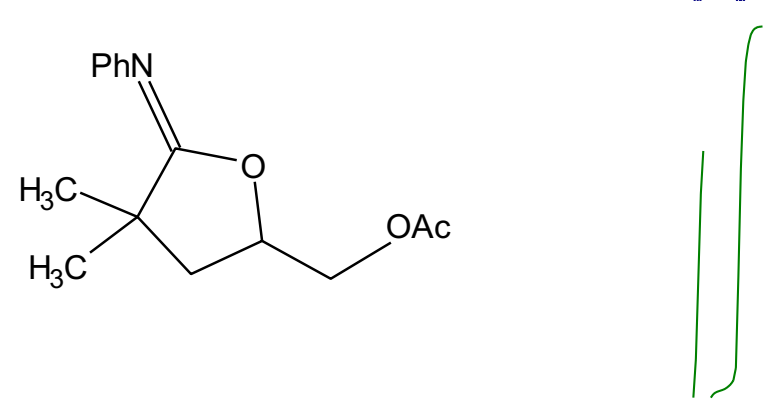

2ah

${ }^{1} \mathrm{H} N M R, 500 \mathrm{MHz}, \mathrm{CDCl}_{3}$
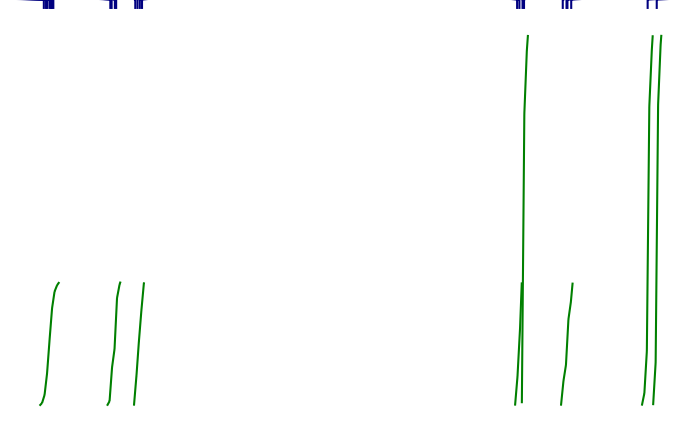

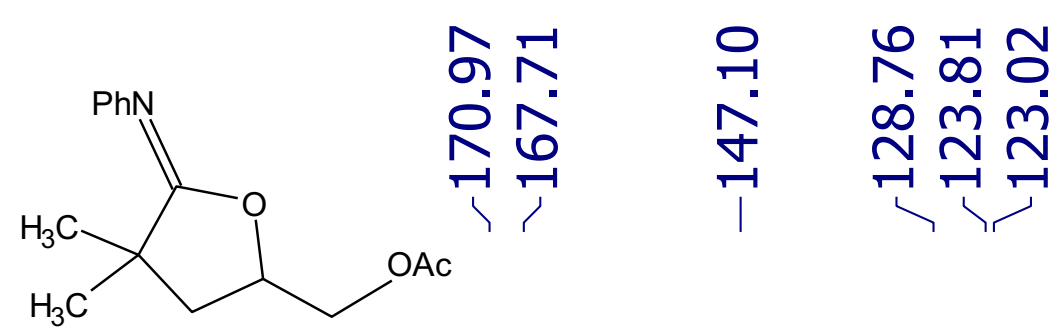

2ah

${ }^{13} \mathrm{C} \mathrm{NMR,}, 126 \mathrm{MHz}, \mathrm{CDCl}_{3}$ 


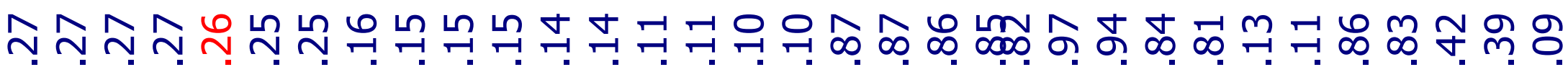

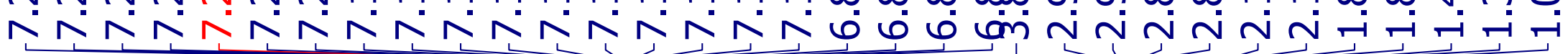
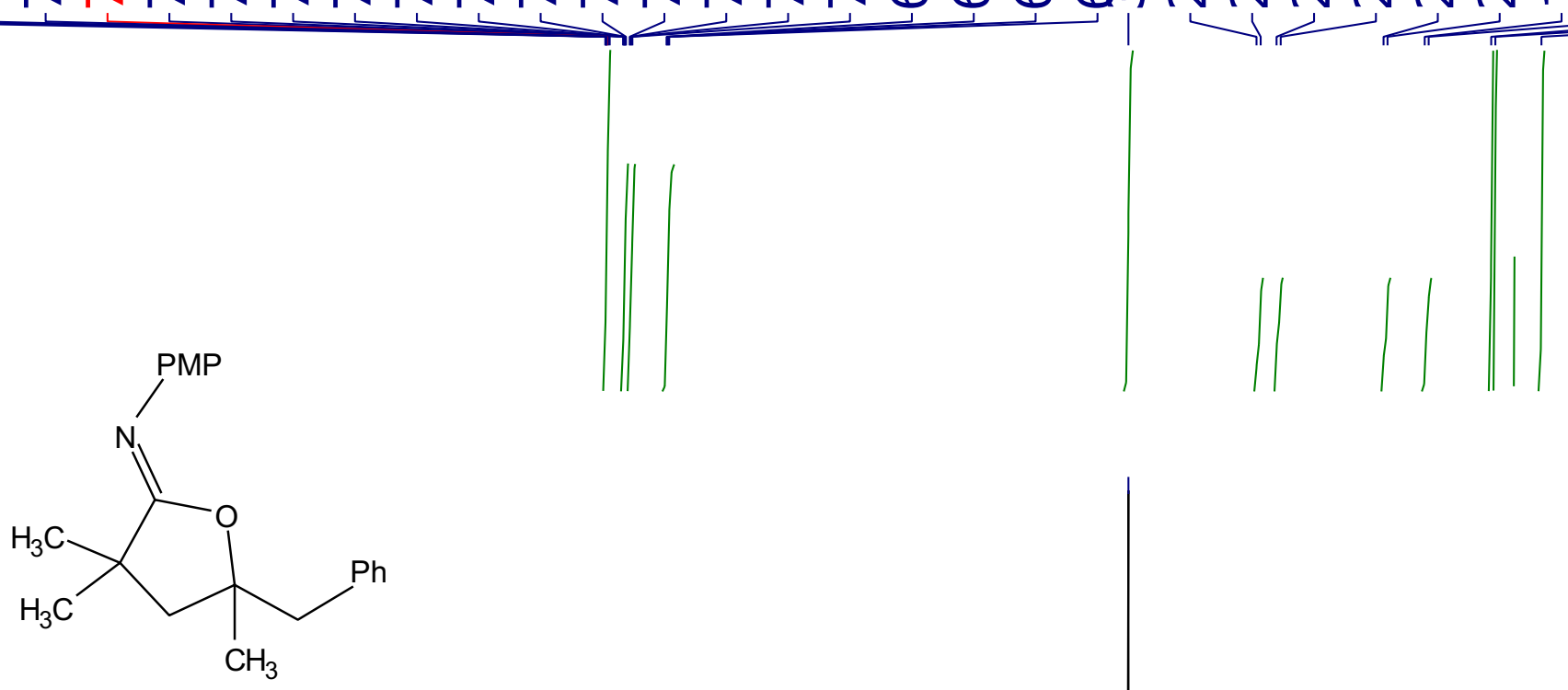

$2 a i$

${ }^{1} \mathrm{HNMR}, 500 \mathrm{MHz}, \mathrm{CDCl}_{3}$
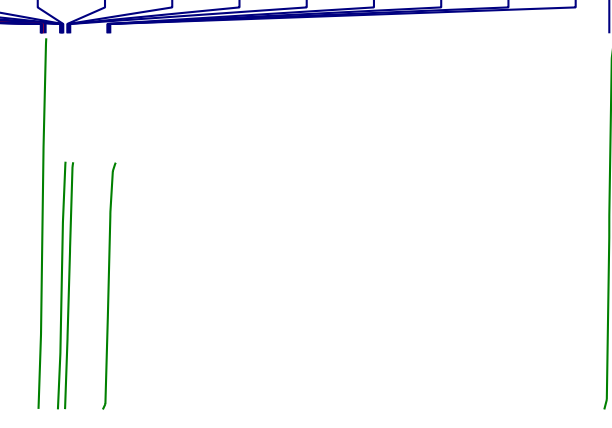


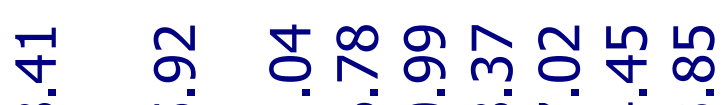

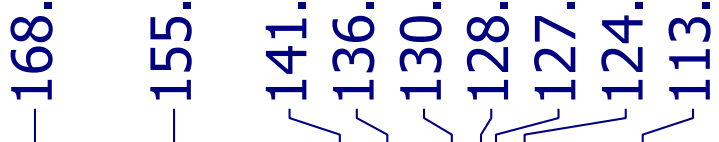

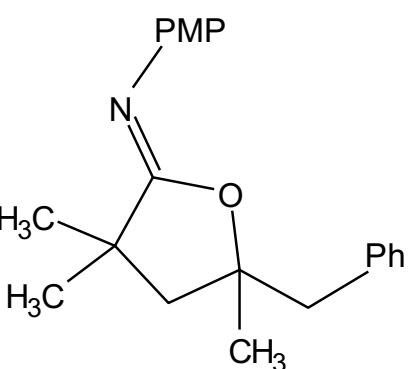

\section{$2 a i$}

${ }^{13} \mathrm{C} \mathrm{NMR}, 126 \mathrm{MHz}, \mathrm{CDCl}_{3}$

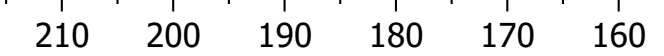

$150 \quad 140$

130 


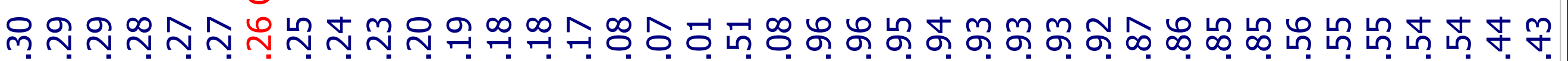

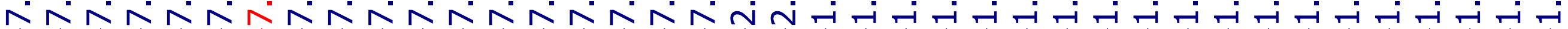

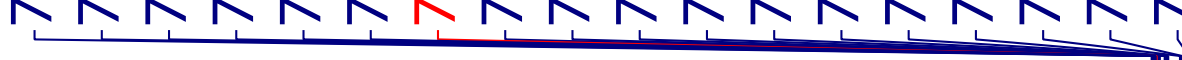
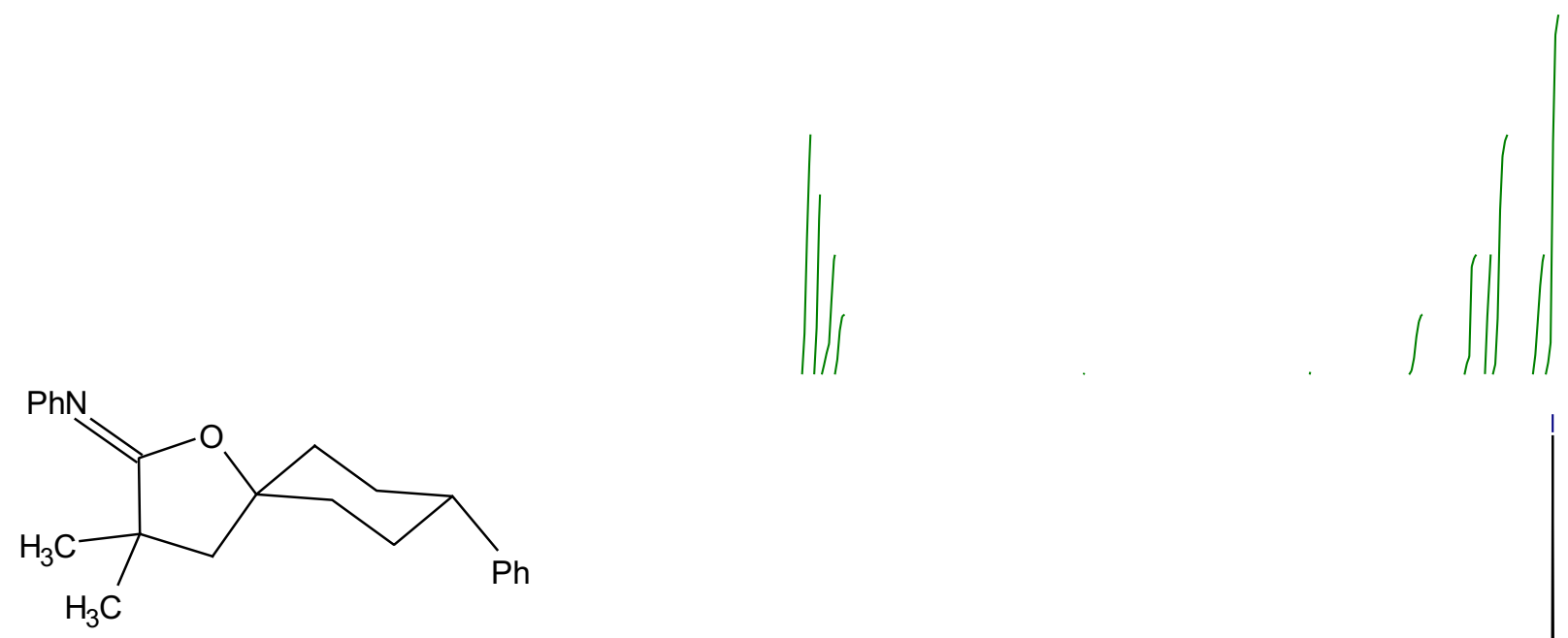

2aj

${ }^{1} \mathrm{H} \mathrm{NMR}, 500 \mathrm{MHz}, \mathrm{CDCl}_{3}$

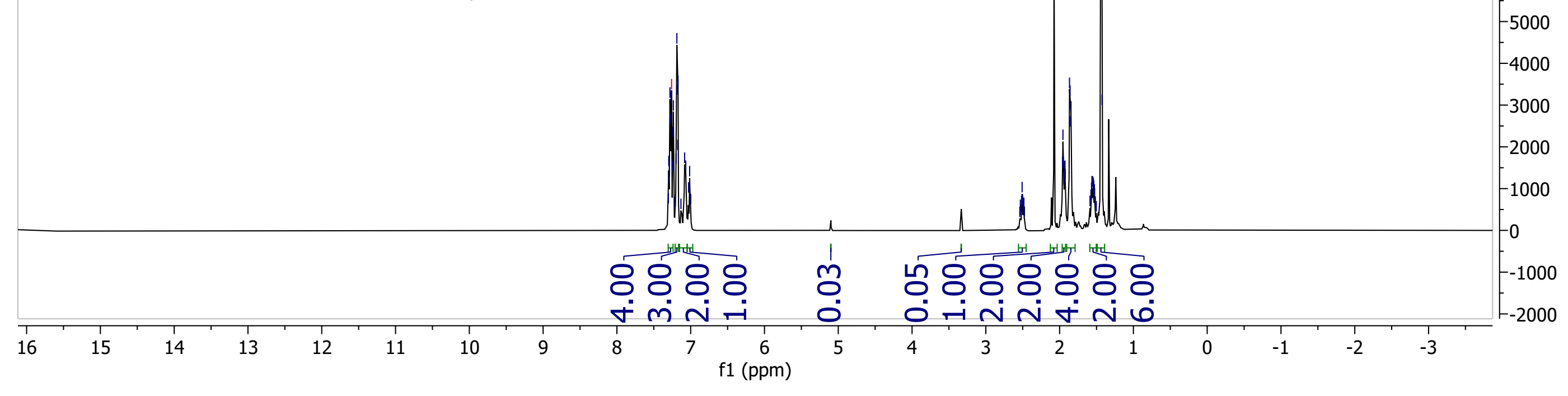




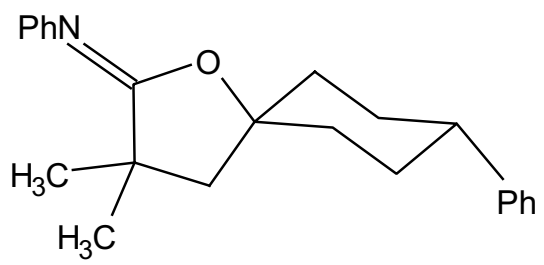

2aj

${ }^{13} \mathrm{C} \mathrm{NMR}, 126 \mathrm{MHz}, \mathrm{CDCl}_{3}$

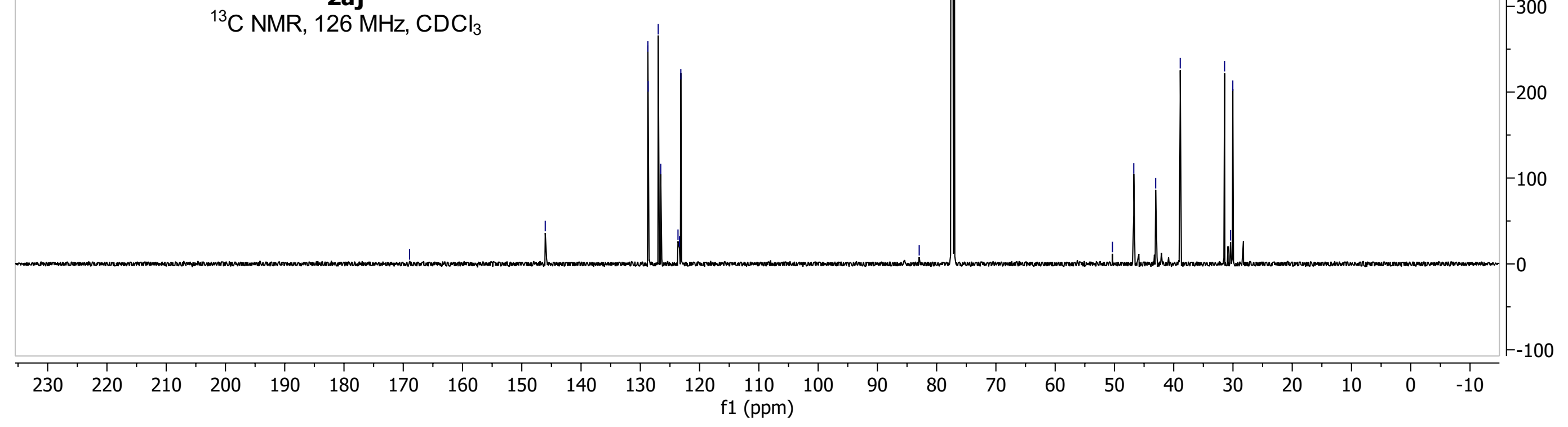


2aj

COSY spectrum

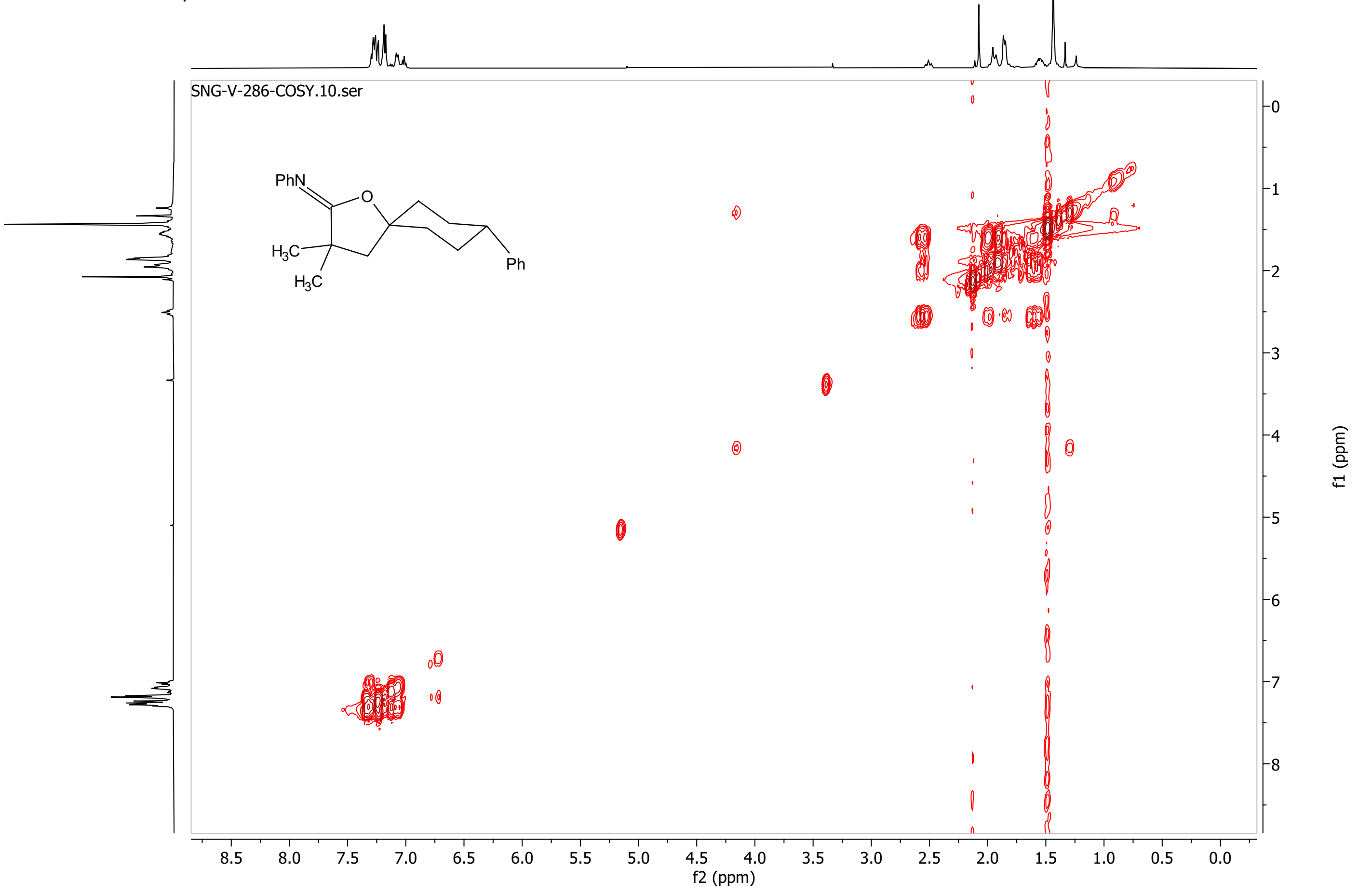


2aj

NOESY spectrum

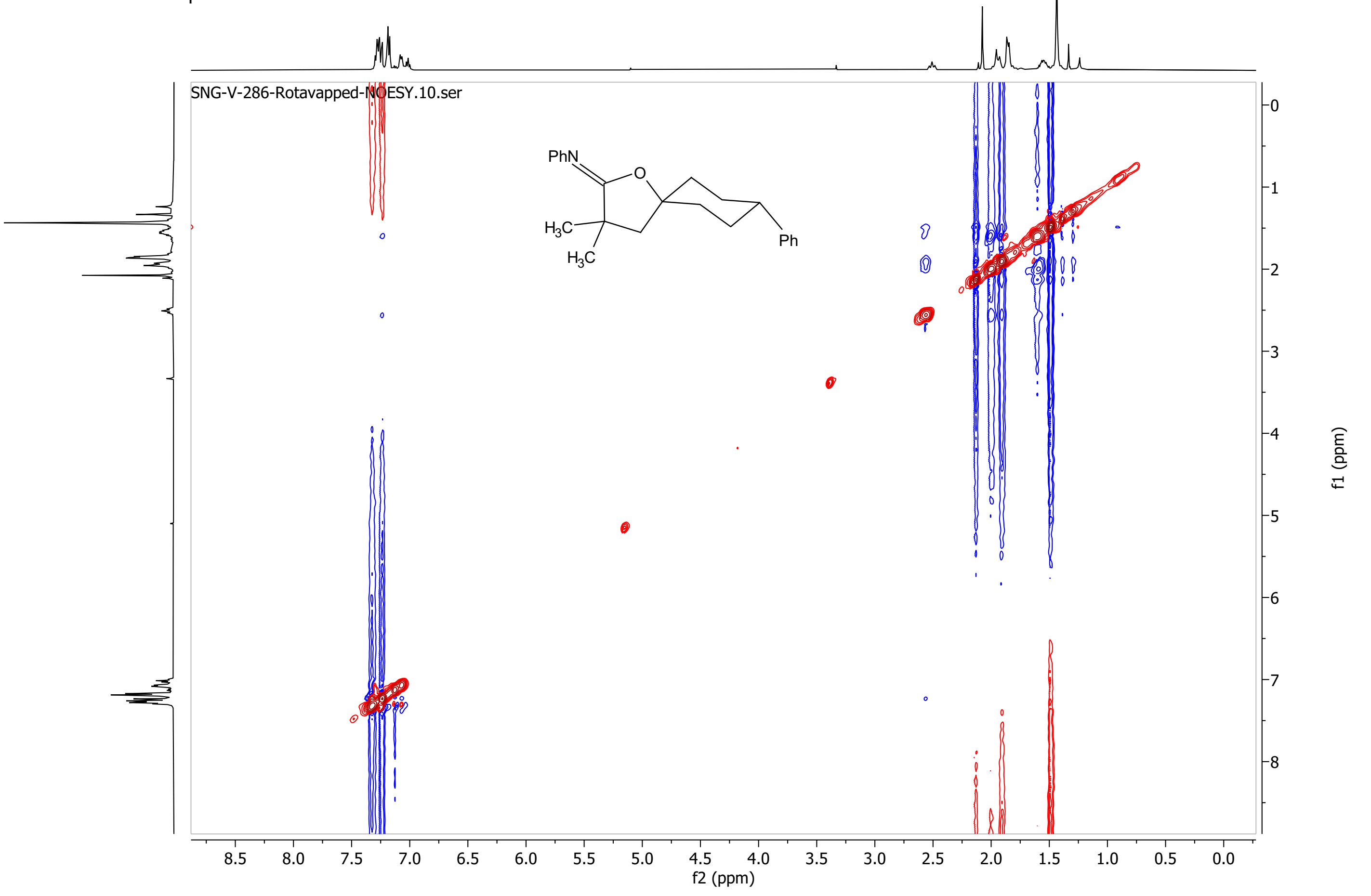




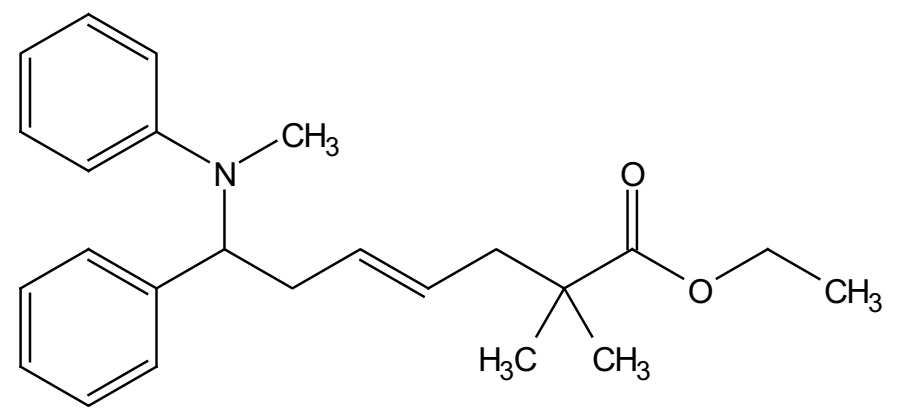

\section{2ak}

${ }^{1} \mathrm{H} N M R, 500 \mathrm{MHz}, \mathrm{CDCl}_{3}$

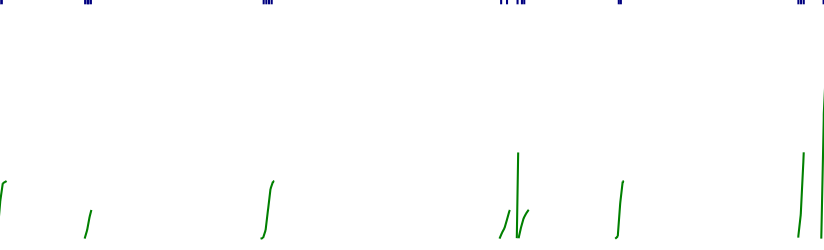




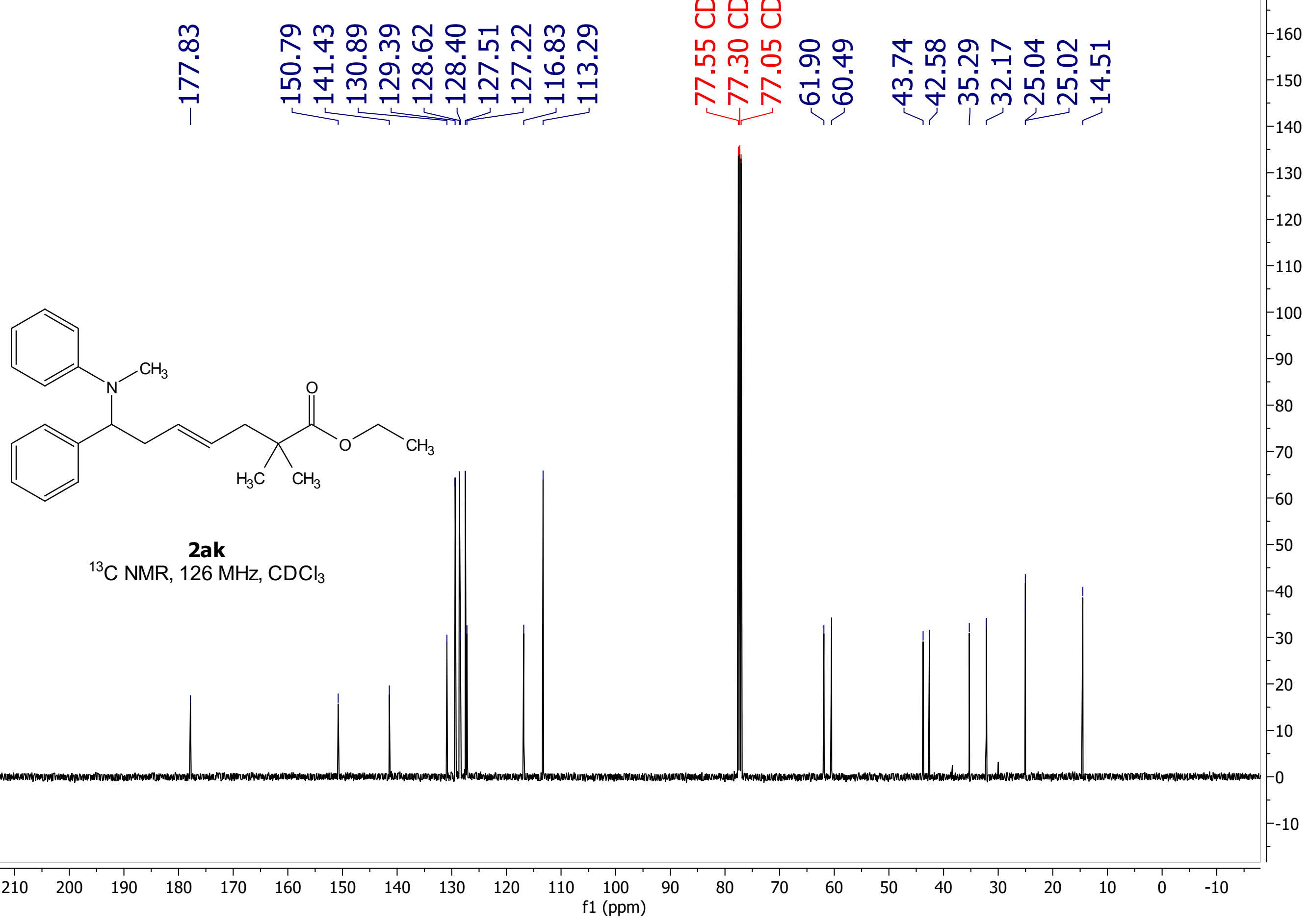

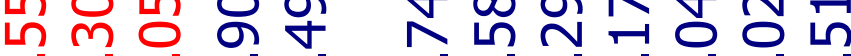
mก ก $\mathrm{N}$ ก 


\section{2ak}

COSY spectrum

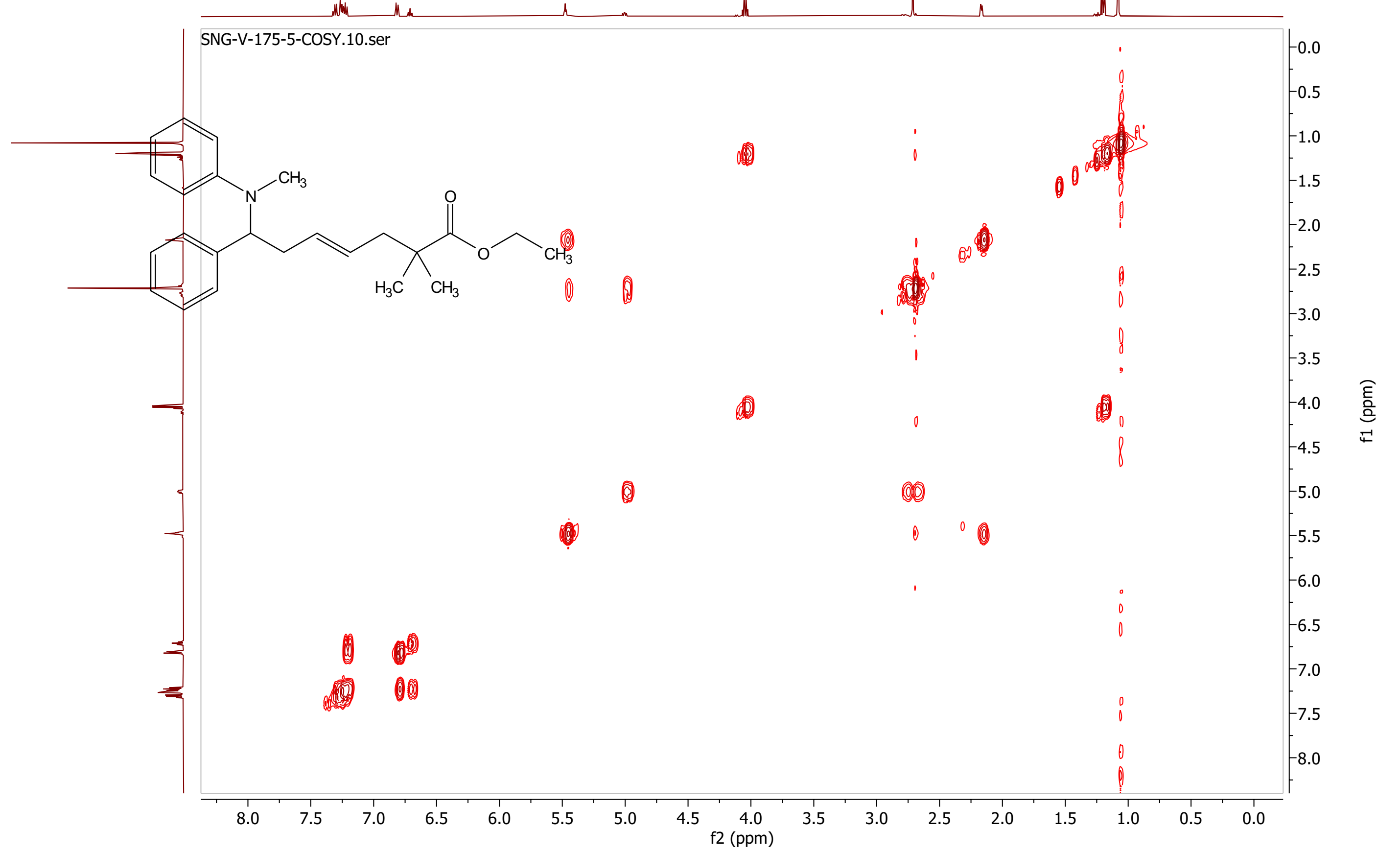




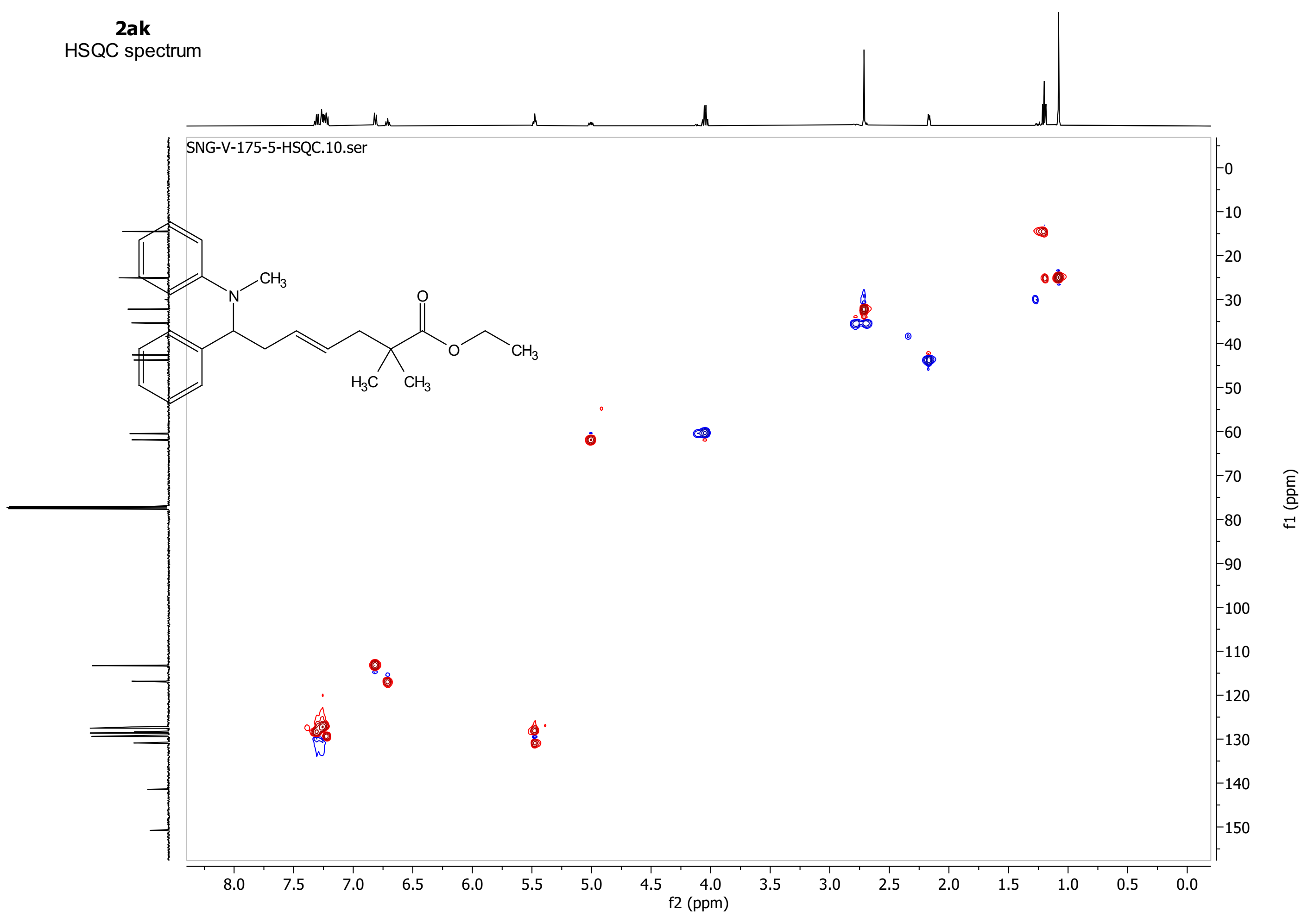


m

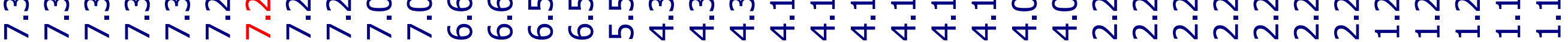

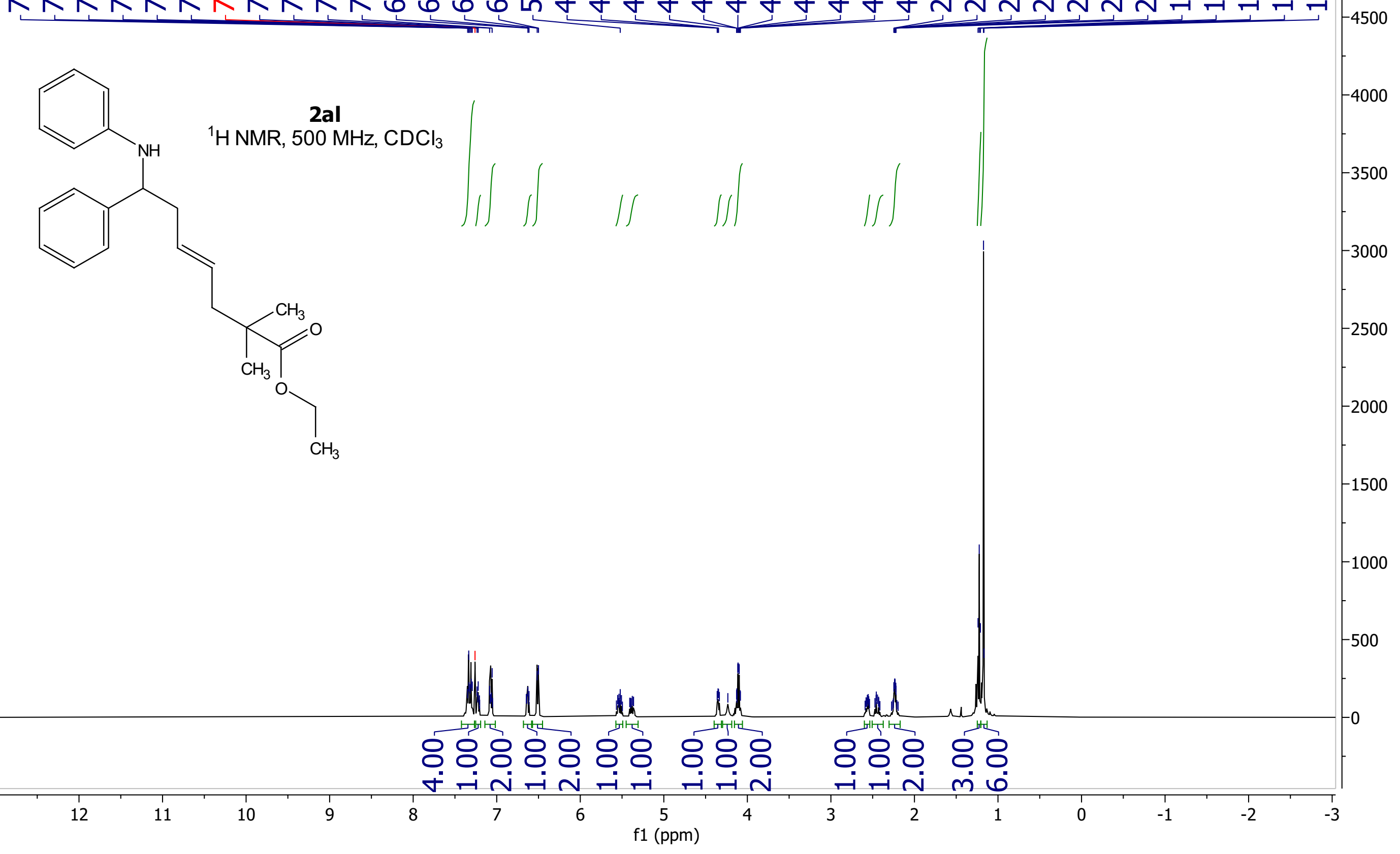




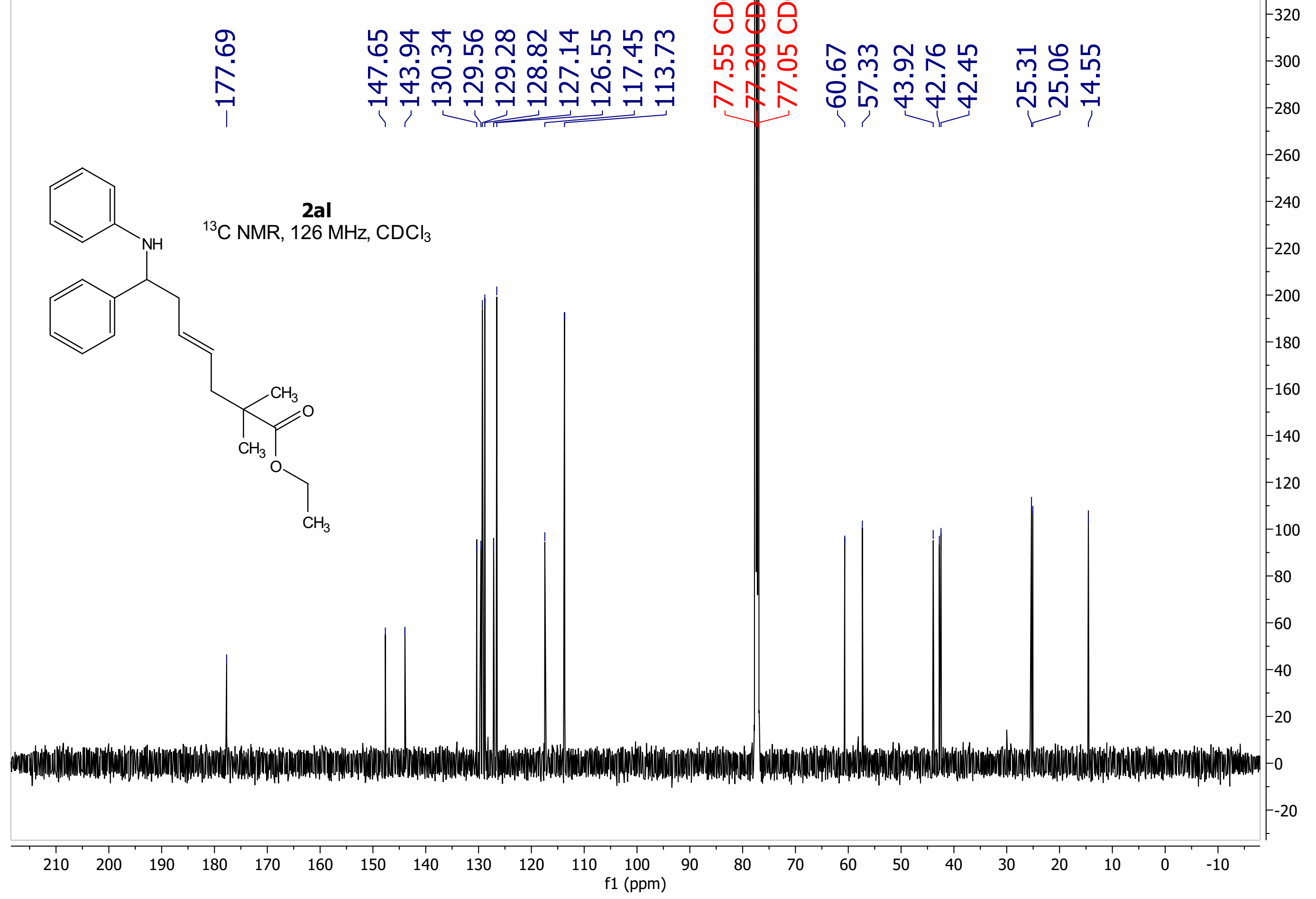


2al

COSY spectrum

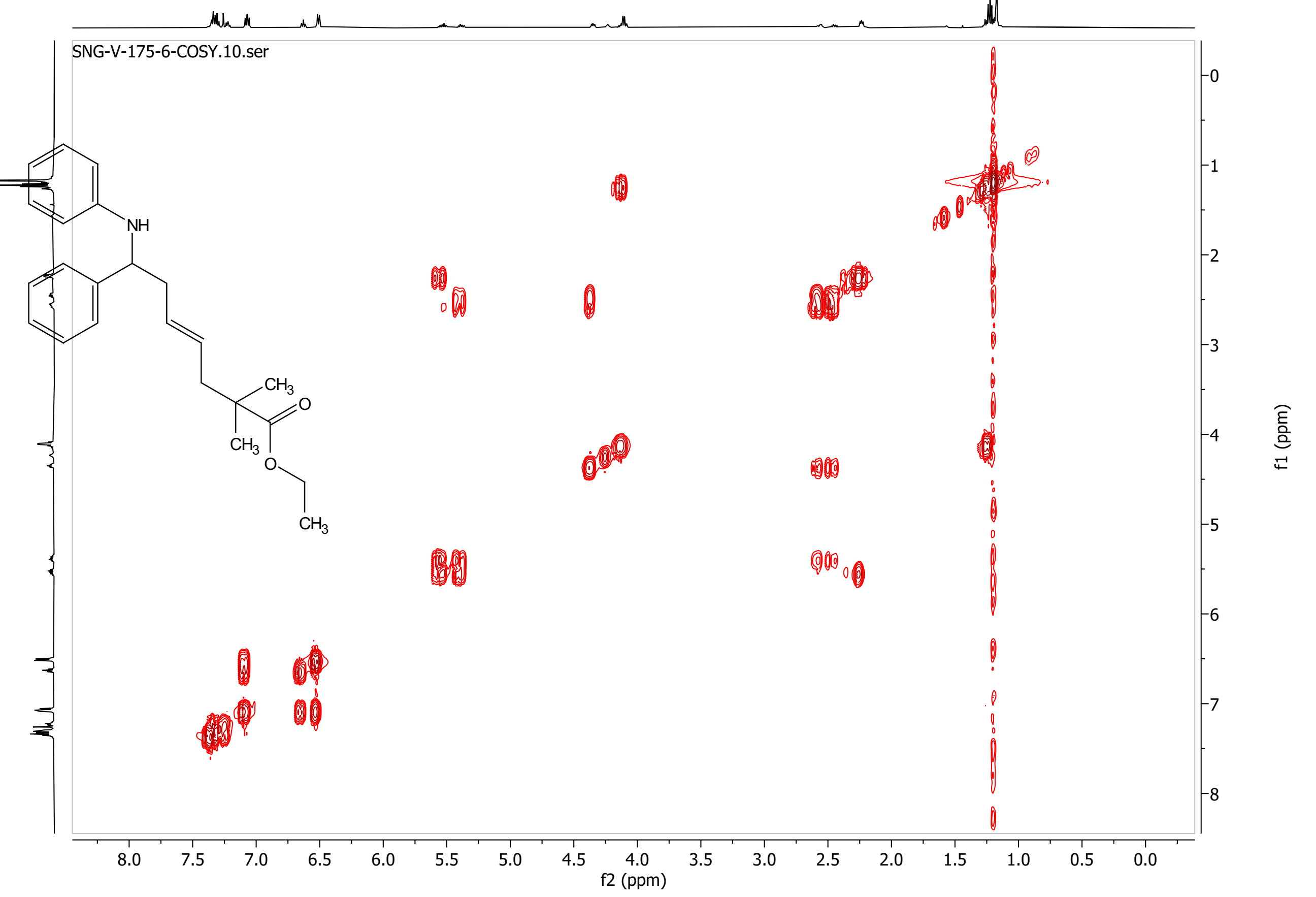




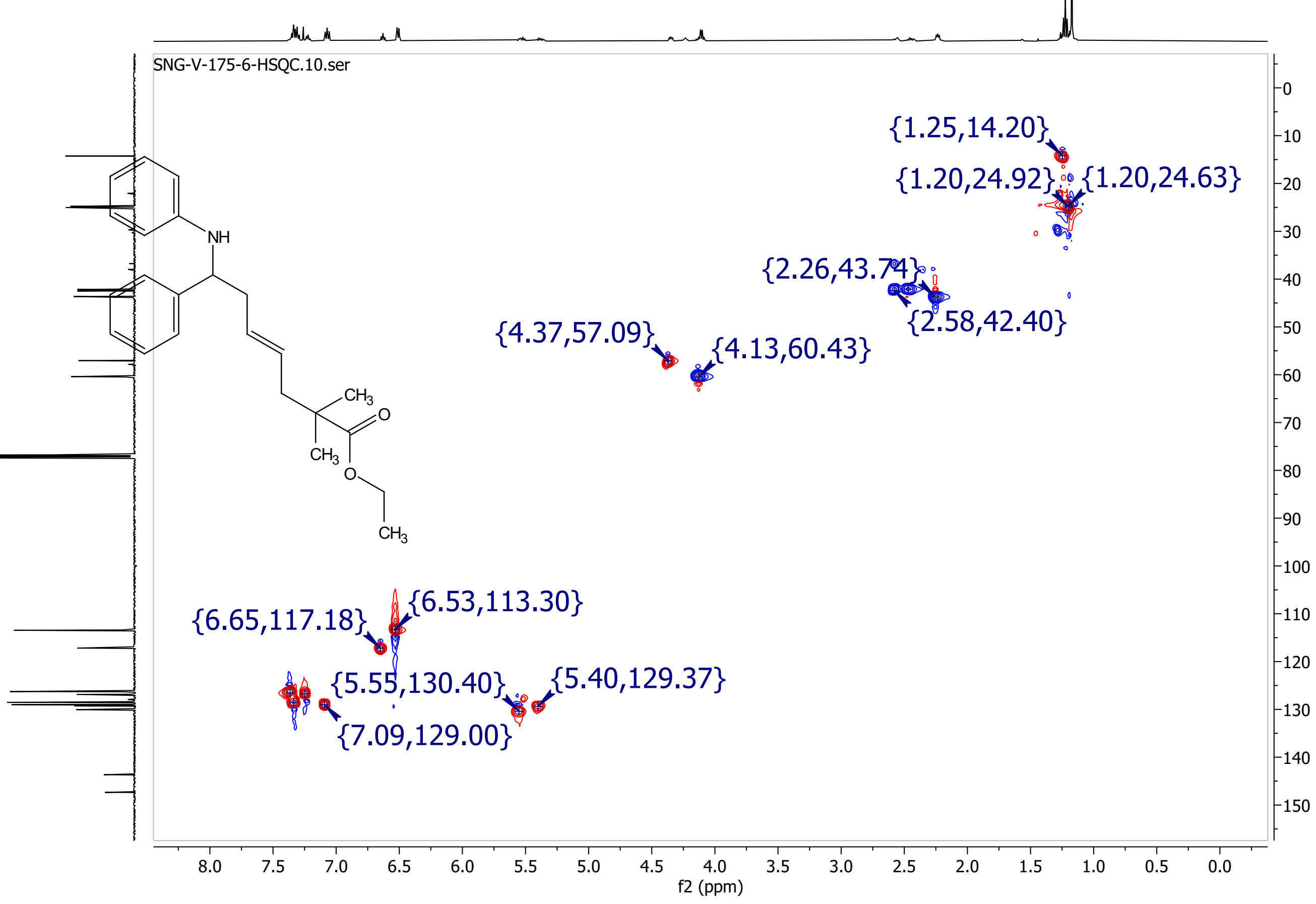


ย

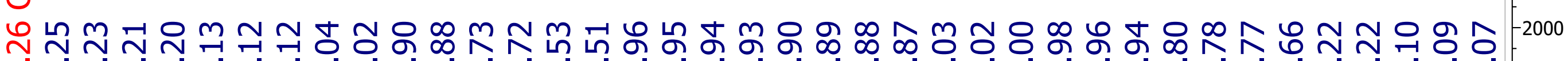

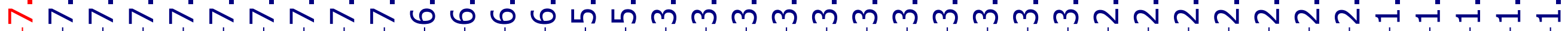<smiles>CCOC(=O)C(C)(C)[C@H]1Cc2ccccc2[C@H]1N(C)c1ccccc1</smiles>

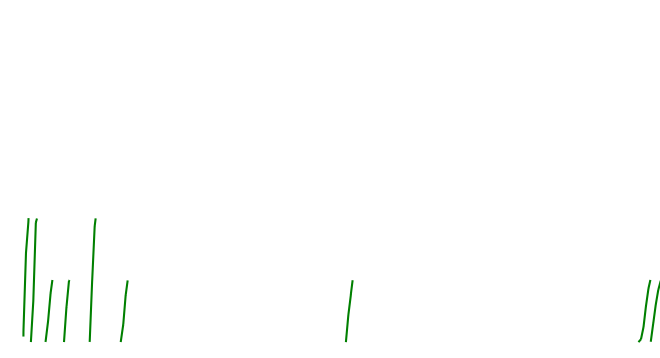

2am

${ }^{1} \mathrm{H} \mathrm{NMR}, 500 \mathrm{MHz}, \mathrm{CDCl}_{3}$
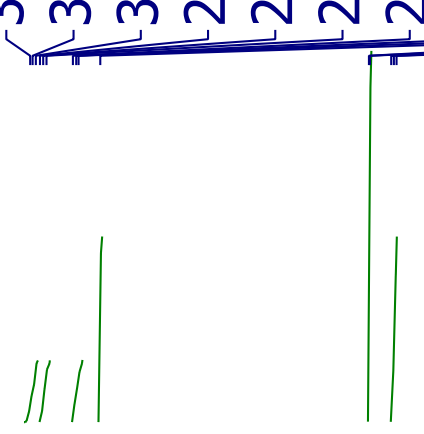

\section{0}

(1)

808080

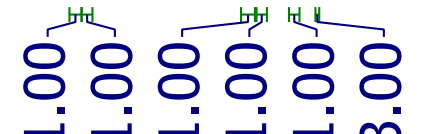

8 


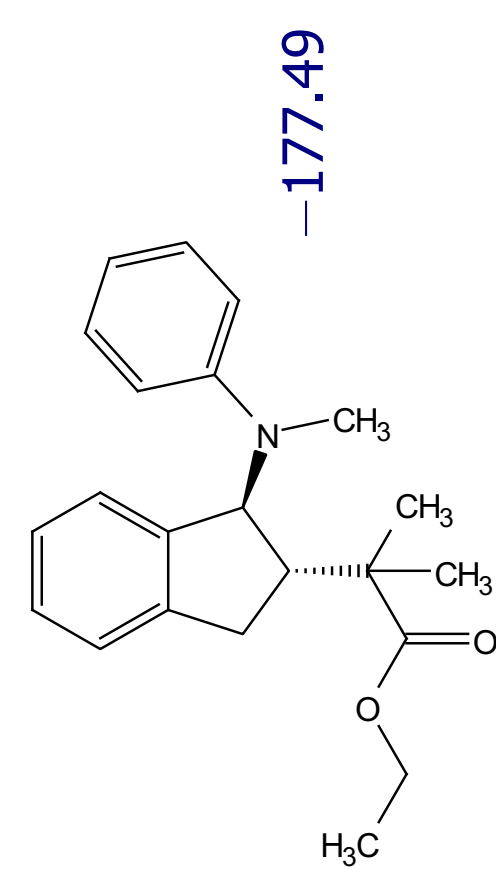

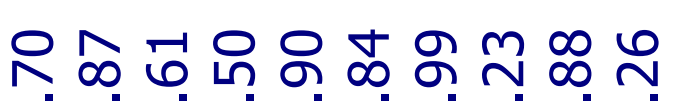

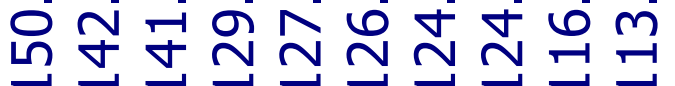

$2 a m$

${ }^{13} \mathrm{C} \mathrm{NMR}, 126 \mathrm{MHz}, \mathrm{CDCl}_{3}$

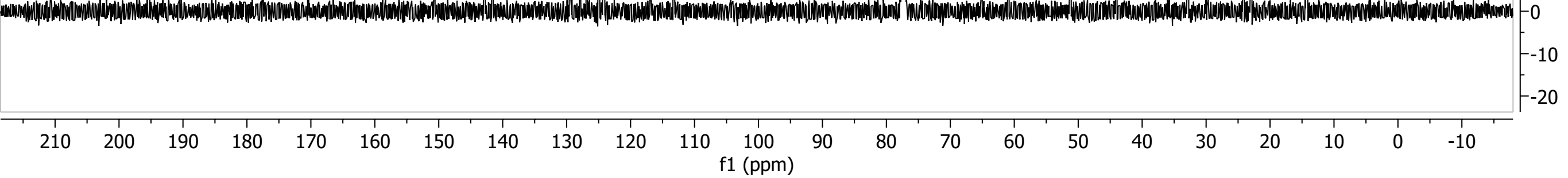


ิㅡํ m

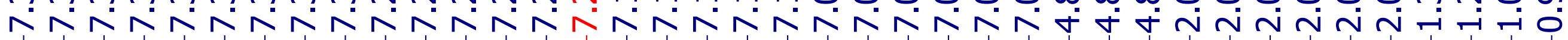
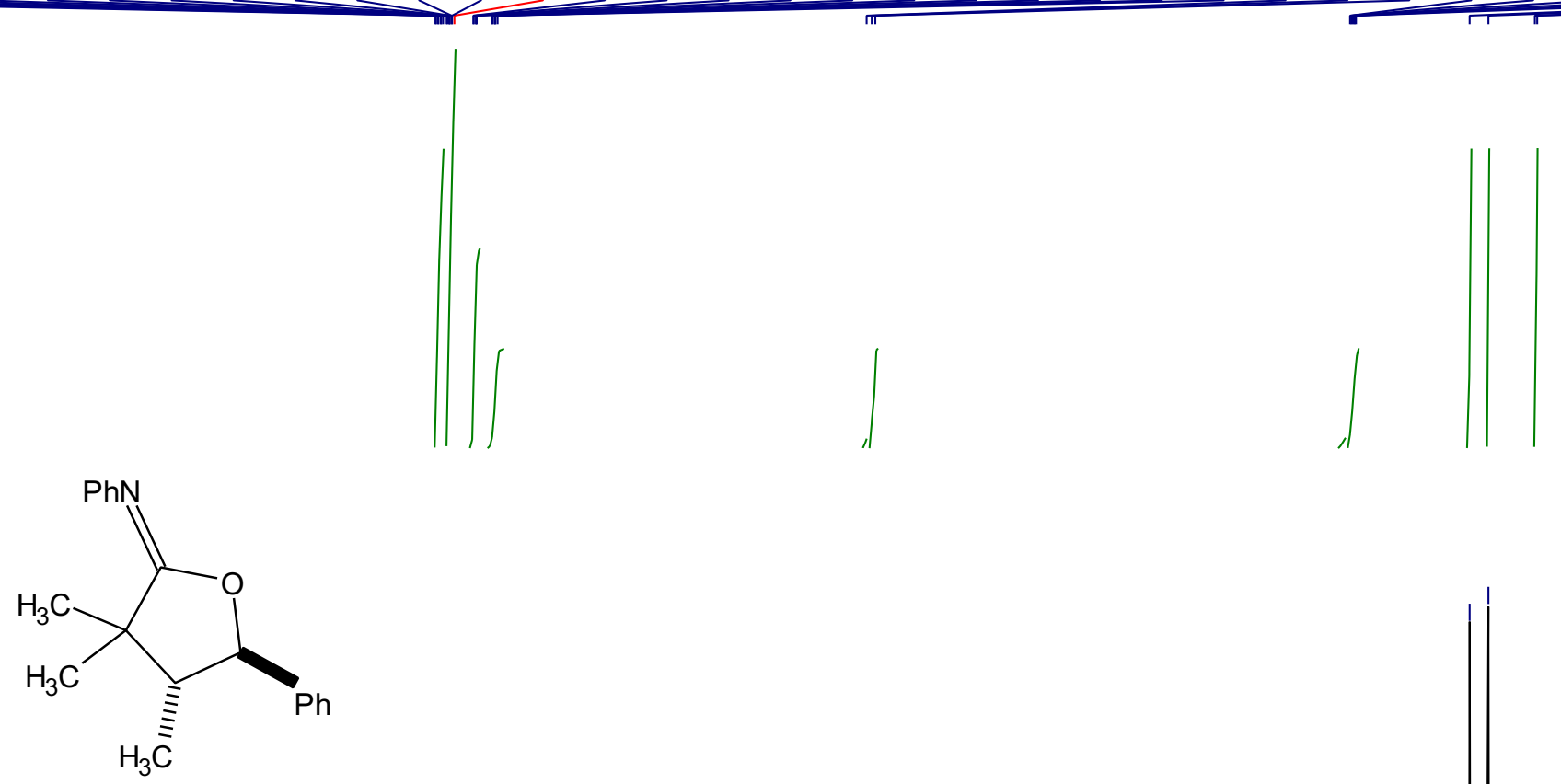

2an (from (Z)- $\beta$-methylstyrene) ${ }^{1} \mathrm{HNMR}, 500 \mathrm{MHz}, \mathrm{CDCl}_{3}$

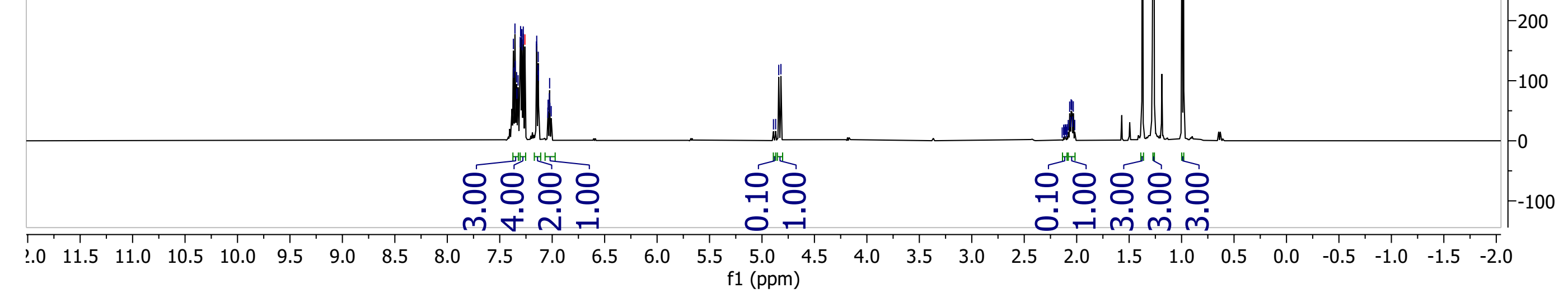




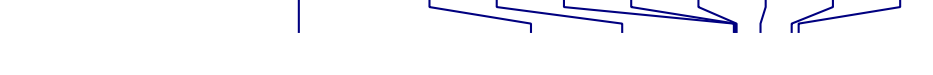<smiles>C[C@H]1[C@H](P)OC(=N[OH2+])C1(C)C</smiles>

2an (from (Z)- $\beta$-methylstyrene)

${ }^{13} \mathrm{C} \mathrm{NMR}, 126 \mathrm{MHz}, \mathrm{CDCl}_{3}$

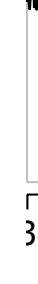




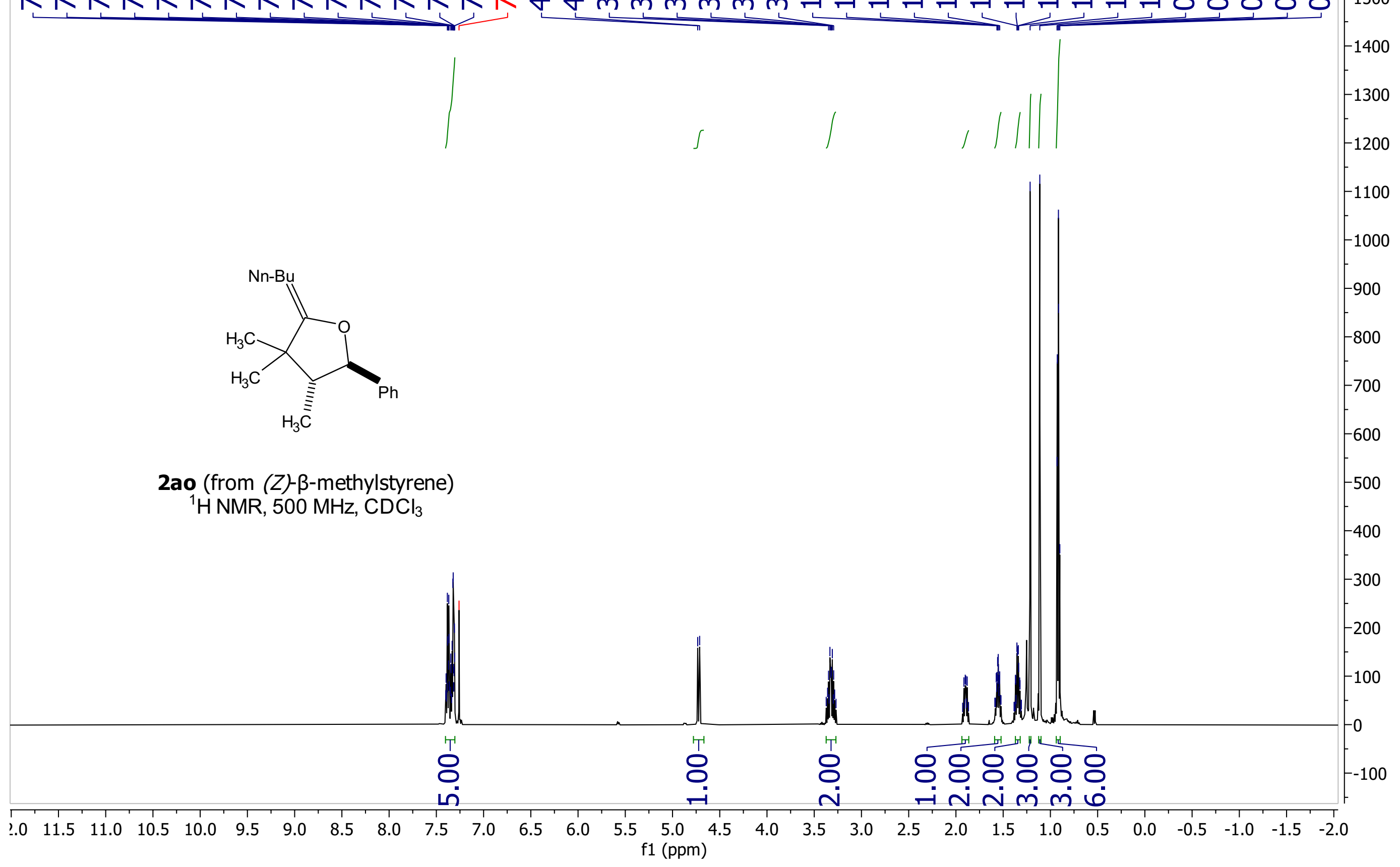




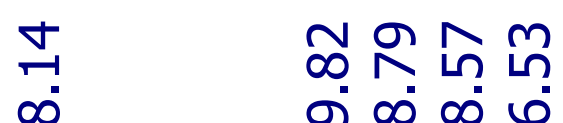

$$
\begin{aligned}
& \text { b } \quad \text { ं } \\
& \text { 각각 }
\end{aligned}
$$

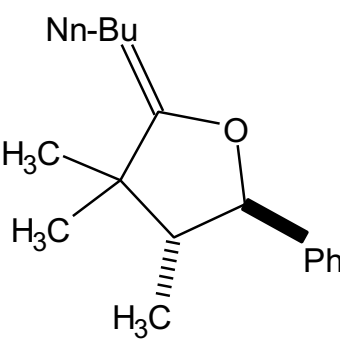

2ao (from (Z)- $\beta$-methylstyrene)

${ }^{13} \mathrm{C} \mathrm{NMR}, 126 \mathrm{MHz}, \mathrm{CDCl}_{3}$

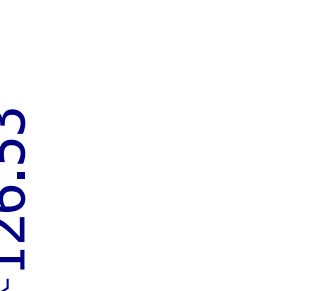

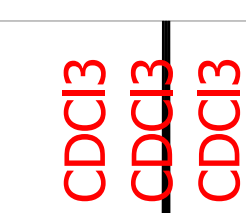
ㄷํ เก ले है மீกN초

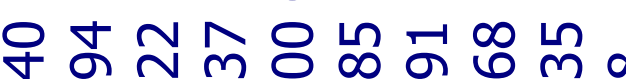
นี 


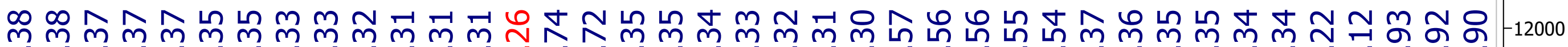

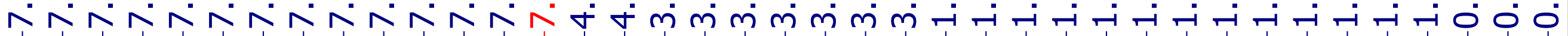

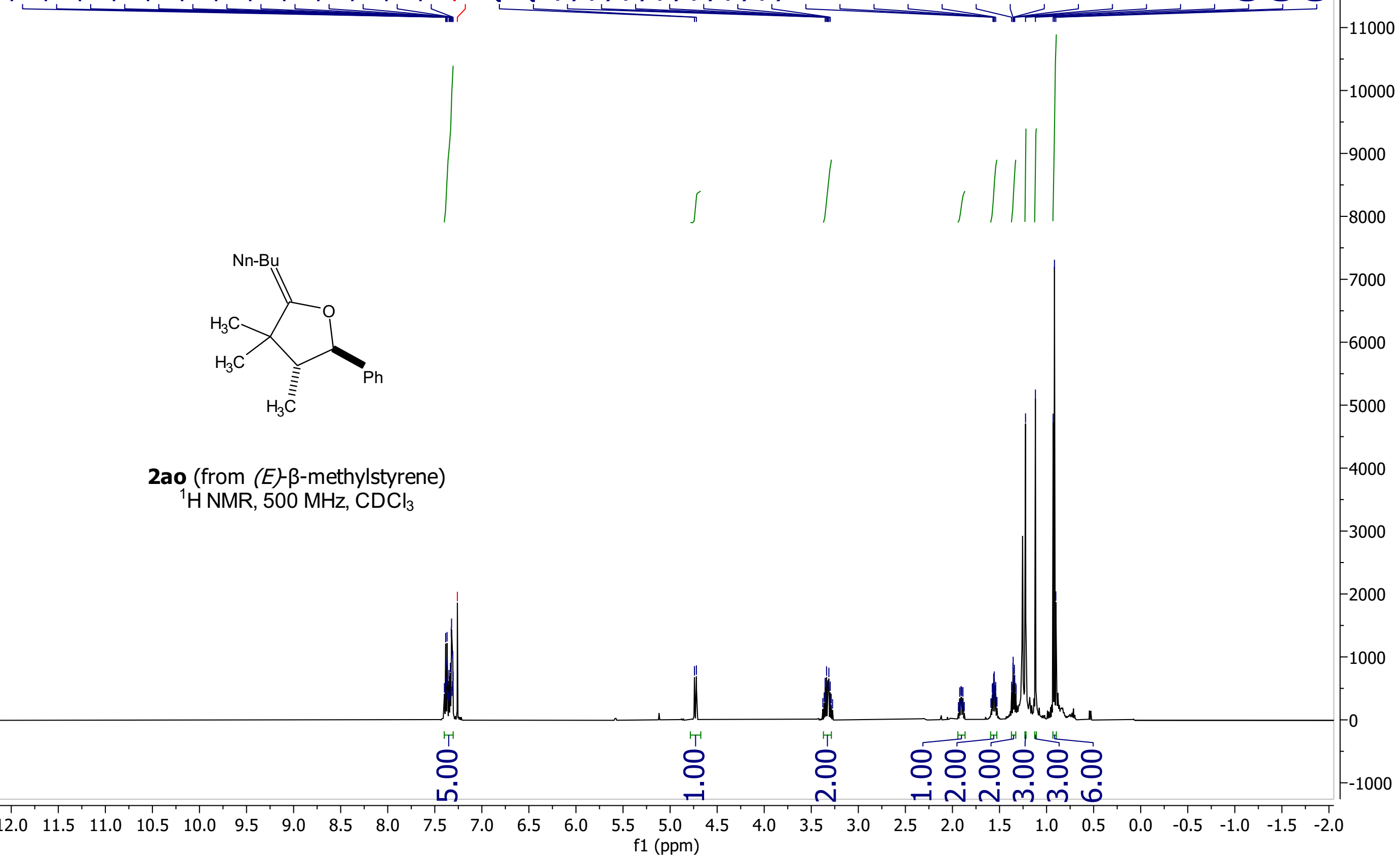




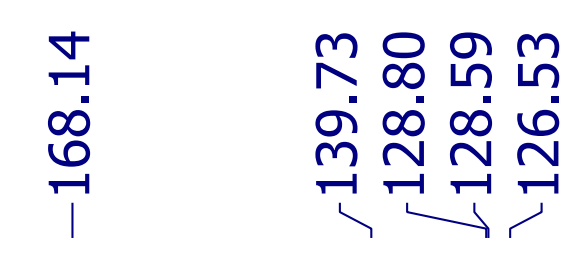

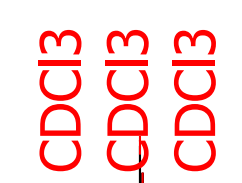

ซึ คำ ำ

ก

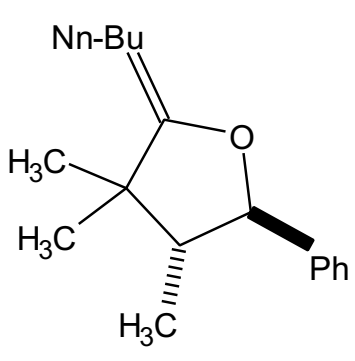

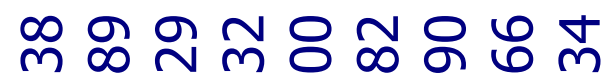
เก

2 ao (from (E)- $\beta$-methylstyrene) ${ }^{1} \mathrm{H} \mathrm{NMR}, 500 \mathrm{MHz}, \mathrm{CDCl}_{3}$ 
<smiles>CCCCCC</smiles>

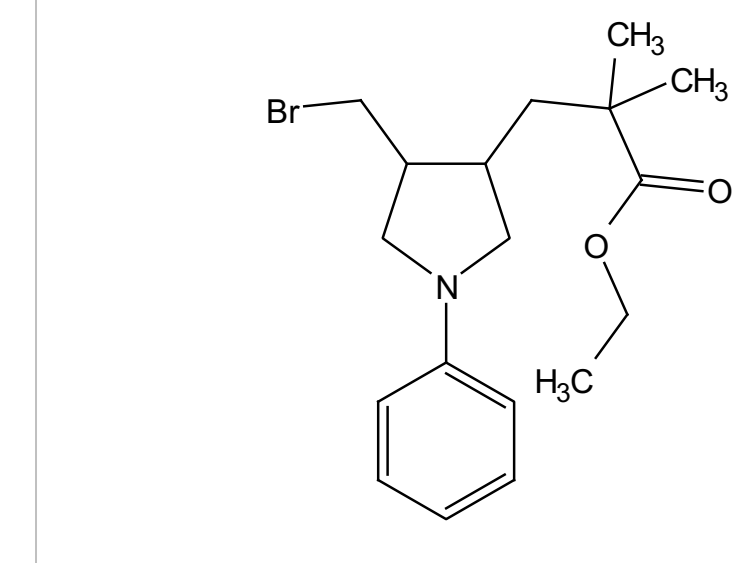

$2 a p$

$1 \mathrm{HNMR}, 500 \mathrm{MHz}, \mathrm{CDCl} 3$

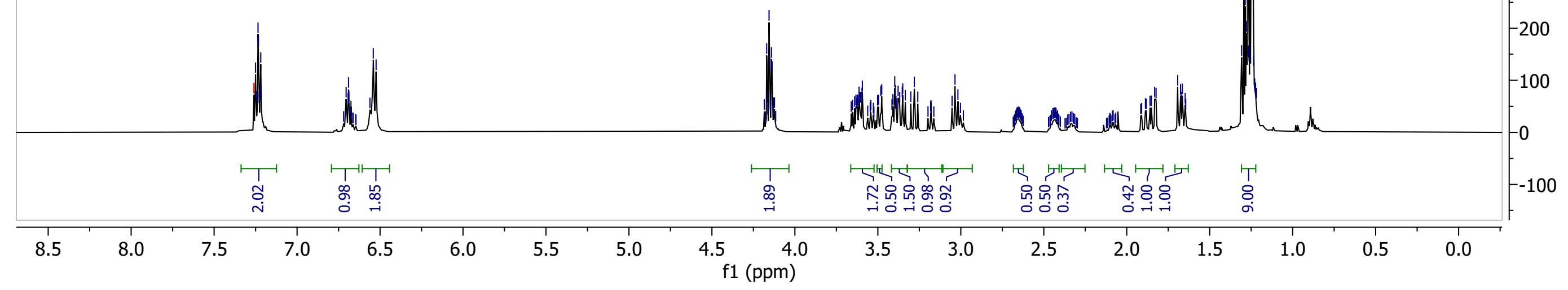


2ap

13C NMR, $126 \mathrm{MHz}, \mathrm{CDCl} 3$

Diastereomer 1

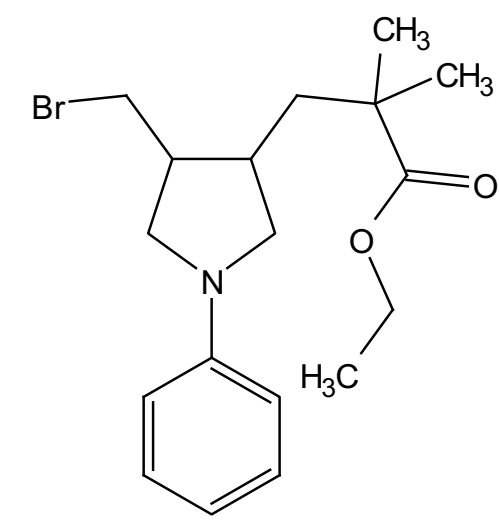


$2 a p$

13C NMR, $126 \mathrm{MHz}, \mathrm{CDCl} 3$

Diastereomer 2

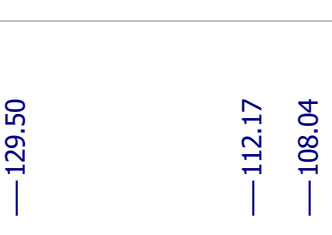

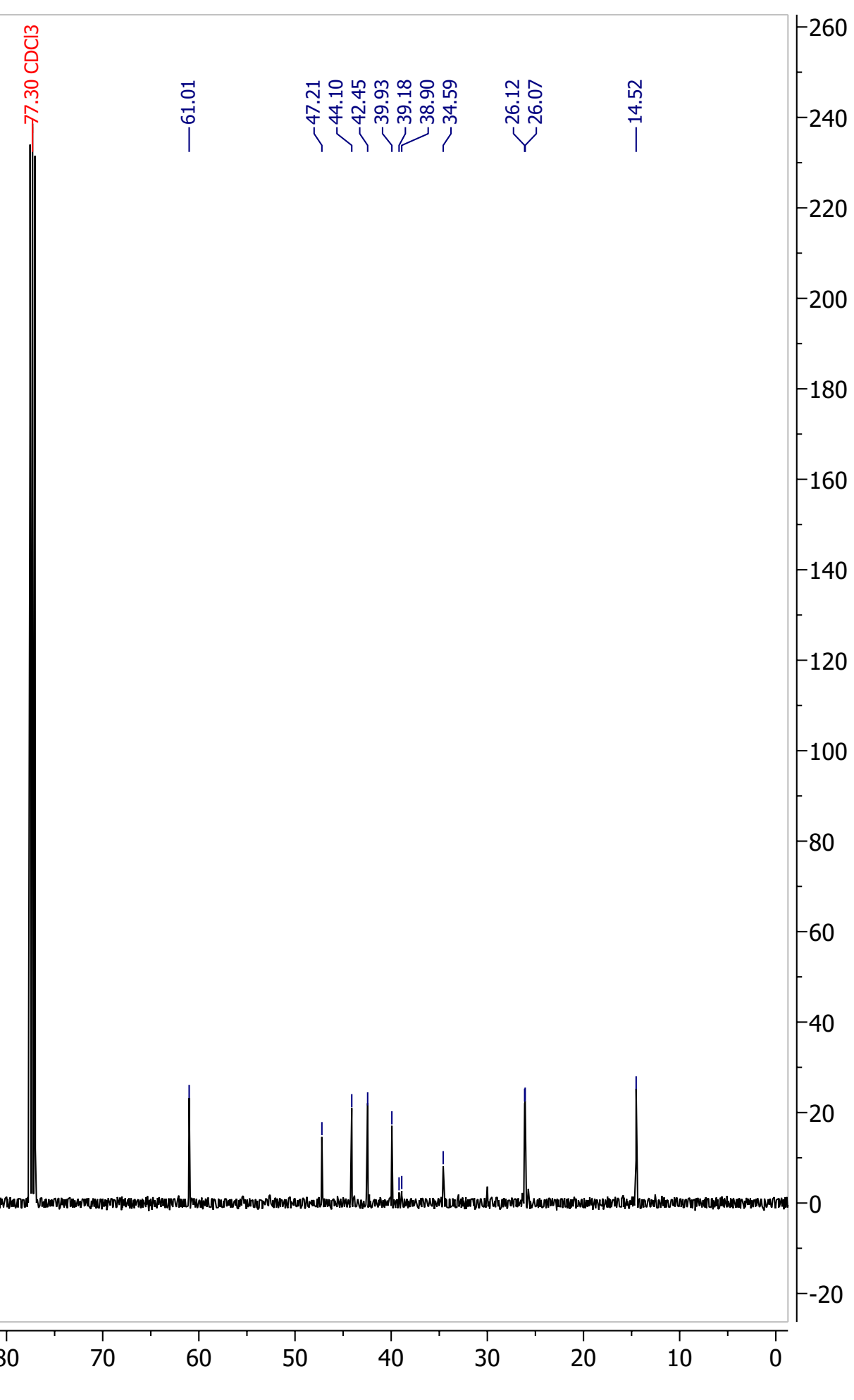

